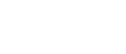
(
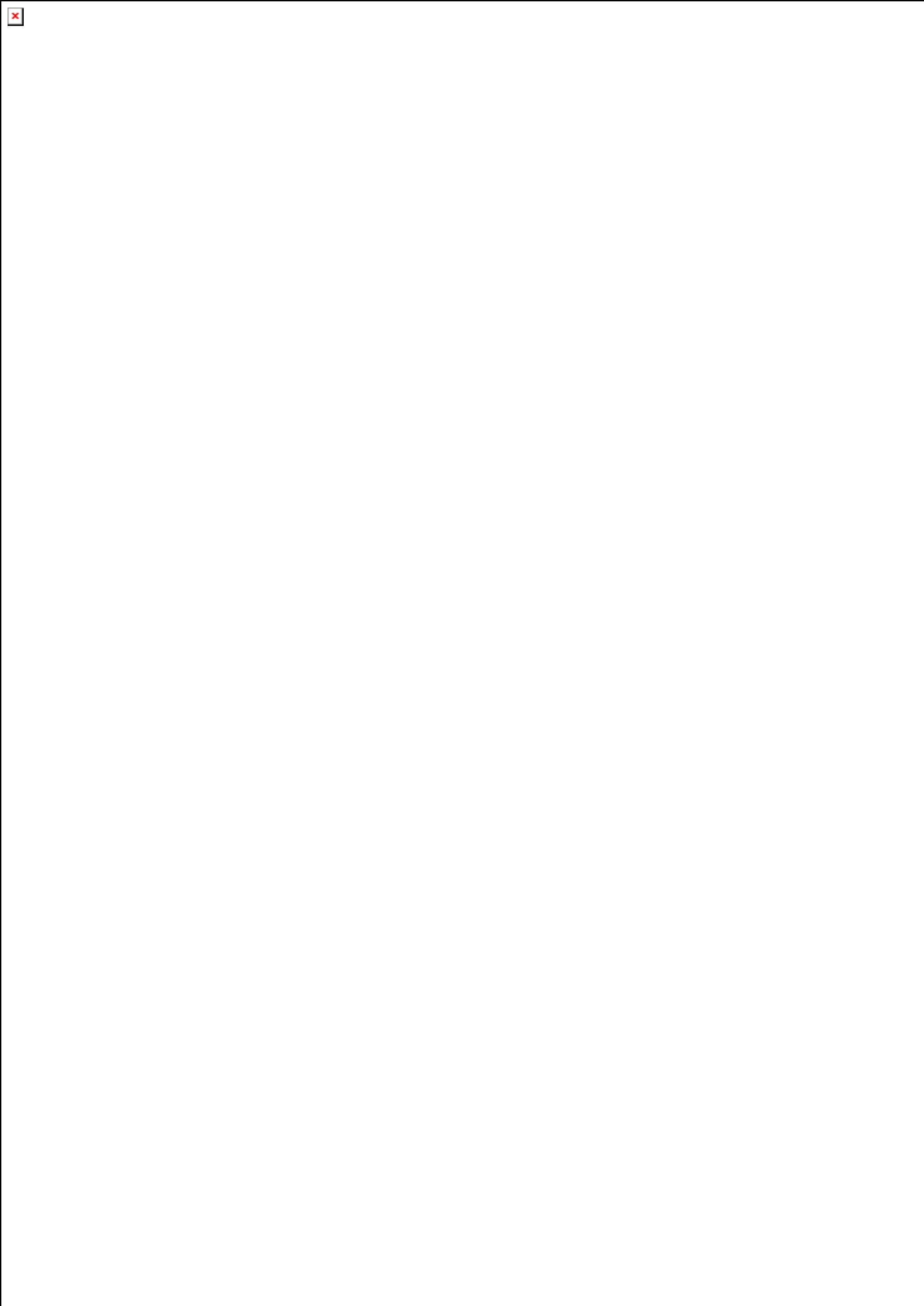





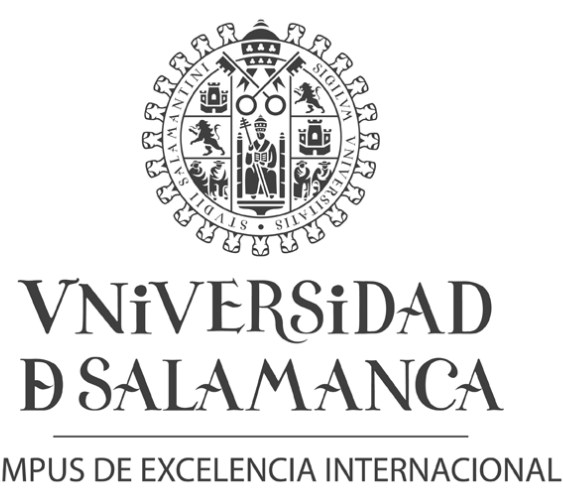

CAMPUS DE EXCELENCIA INTERNACIONAL

\section{Reconstrucción de la dinámica oceanográfica, productividad de cocolitóforos y salinidad en el mar Mediterráneo occidental desde el Último Máximo Glacial}

\footnotetext{
Memoria presentada por Blanca Ausín González para optar al grado de Doctora en Geología por la Universidad de Salamanca con mención

"Doctor Europeus"
}

Directores de la Tesis:

Dr. José Abel Flores Villarejo

Catedrático del Departamento de Geología (Paleontología)

Universidad de Salamanca

Dr. Francisco Javier Sierro Sánchez

Catedrático del Departamento de Geología (Paleontología)

Universidad de Salamanca 

D. José Abel Flores Villarejo y D. Francisco Javier Sierro Sánchez, profesores del Área de Paleontología en el Departamento de Geología de la Facultad de Ciencias (Universidad de Salamanca)

CERTIFICAN:

Que Blanca Ausín González ha realizado en el Departamento de Geología de la Universidad de Salamanca y bajo nuestra supervisión el trabajo:

Reconstrucción de la dinámica oceanográfica, productividad de cocolitóforos y salinidad en el mar Mediterráneo occidental desde el Último Máximo

Glacial

Y para que así conste, firmamos el presente certificado en Salamanca, en mayo de 2015:

Los directores:

Dr. José Abel Flores Villarejo

Dr. Francisco Javier Sierro Sánchez

La doctoranda:

Blanca Ausín González 
La realización de esta tesis ha sido posible gracias a la concesión de la beca AP2010-2559 de Formación del Personal Universitario (FPU) del Ministerio de Educación, Cultura y Deporte y a la financiación de los proyectos PASUR CGL2009-08651 y VACLIODP339 CTM2012-38248 del Ministerio de Economía y Competitividad. Adicionalmente se ha obtenido financiación de la Universidad de Salamanca a través del Programa de Difusión de Resultados de la Agencia de Investigación. 
- Ama usted el mar, Capitán.

- ¡Sí! ¡Lo amo! ¡El mar es todo! Cubre las siete décimas partes del globo terrestre. [...] En el mar está la suprema tranquilidad. El mar no pertenece a los déspotas. En su superficie pueden todavía ejercer sus derechos inicuos, batirse, entredevorarse, transportar a ella todos los horrores terrestres. Pero a treinta pies de profundidad, su poder cesa, su influencia se apaga, su potencia desaparece. ¡Ah! ¡Viva usted, señor, en el seno de los mares, viva en ellos! Solamente abi está la independencia. ;Abi no reconozco dueño ni señor! ; Abi yo soy libre!

20.000 Leguas de Viaje Submarino [Julio Verne] 
ÍNDICE

Resumen I

Abstract

PRESENTACIÓN Y JUSTIFICACIÓN V V

HIPÓTESIS VI

OBJETIVOS VI

ESTRUCTURA DE LA TESIS DOCTORAL VII

Referencias IX

CAPÍTULO 1.-DINÁMINA OCEÁNICA Y ATMOSFÉRICA DE LA ZONA DE ESTUDIO

1.1.-EL MAR MEDITERRÁNEO 1

1.2.-EL MAR MEDITERRÁNEO OCCIDENTAL 2

1.2.1.-Dinámica oceánica actual 2

1.3.-EL MAR DE ALBORÁN 6

1.3.1.-Hidrografía del mar de Alborán 6

$\begin{array}{ll}\text { 1.3.2.-Productividad en el mar de Alborán } & 7\end{array}$

1.3.3.-Importancia de los factores atmosféricos en el mar de Alborán $\quad 10$

1.4.-TELECONEXIÓN ATMOSFÉRICA CON EL ATLÁNTICO NORTE 10

Referencias $\quad 12$

\section{CAPÍTULO 2.-COCOLITÓFOROS}

$\begin{array}{ll}\text { 2.1.-INTRODUCCIÓN } & 19\end{array}$

2.2.-ESTRUCTURA CELULAR 20

$\begin{array}{ll}\text { 2.2.1.-Cocolitos y sus tipologías } & 21\end{array}$

2.3.-CICLO DE VIDA 24

2.4.-ECOLOGÍA Y DISTRIBUCIÓN 25

2.4.1.-Factores limitantes $\quad 25$

2.4.2.-Distribución espacial 25

2.4.3.-Distribución vertical $\quad 27$

2.4.4.-Variabilidad espacial y temporal de la distribución de los cocolitóforos 27

2.5.-DE LA ZONA FÓTICA AL REGISTRO FÓSIL: 28

2.5.1.-Transporte 28

2.5.2.-Sedimentación $\quad 30$

$\begin{array}{ll}\text { 2.5.3.-Preservación } & 30\end{array}$

2.6.-SISTEMÁTICA DE LOS TAXONES ESTUDIADOS 31

2.7.-CARACTERÍSTICAS Y PREFERENCIAS ECOLÓGICAS DE LOS TAXONES ESTUDIADOS 32

Referencias $\quad 39$

\section{CAPÍTULO 3.-MATERIALES Y TÉCNICAS}

\begin{tabular}{lr}
\hline 3.1.-MATERIALES & 49 \\
3.1.1.-Testigo CEUTA10PC08 & 49 \\
3.1.2.-Testigo HER-GC-T1 & 53 \\
3.1.3.-Base de datos actual & 56
\end{tabular}


3.2.1.-Análisis micropaleontológico para el estudio de las asociaciones cocolitoforales

3.2.2.-Análisis de isótopos de oxígeno

3.2.3.-Análisis de compuestos orgánicos $\quad 63$

3.2.4.- Técnicas estadísticas $\quad 65$

$\begin{array}{ll}\text { Referencias } & 71\end{array}$

\section{CAPÍTULO 4.- COCCOLITHOPHORE PRODUCTIVITY AND SURFACE WATER DYNAMICS IN THE ALBORAN SEA DURING THE LAST 25 KYR}

$\begin{array}{ll}\text { Resumen } & 78\end{array}$

$\begin{array}{ll}\text { Abstract } & 79\end{array}$

$\begin{array}{lr}\text { 4.1.-INTRODUCTION } & 80\end{array}$

4.2.-STUDY AREA AND OCEANOGRAPHIC SETTING 81

4.3.-MATERIALS AND METHODS

$\begin{array}{ll}\text { 4.3.1.-Age model } & 82\end{array}$

$\begin{array}{lr}\text { 4.3.2.-Coccolithophore assemblage analysis } & 84\end{array}$

$\begin{array}{ll}\text { 4.3.3.-Oxygen stable isotopes } & 84\end{array}$

$\begin{array}{ll}\text { 4.3.4.-Planktonic foraminifera-based SST reconstruction } & 85\end{array}$

4.3.5.-Molecular biomarkers and $\mathrm{U}^{\mathrm{k}^{\prime}}{ }_{37}$-based SST $\quad 86$

4.3.6.-Statistical analysis $\quad 86$

$\begin{array}{lr}\text { 4.4.-RESULTS } & 87\end{array}$

$\begin{array}{lr}\text { 4.4.1.-Coccolithophore assemblages } & 87\end{array}$

$\begin{array}{lr}\text { 4.4.2.-Oxygen isotope record } & 90\end{array}$

4.4.3.-Sea surface temperature (SST) 90

4.4.3.1.-Planktonic foraminifera-based SST reconstruction: calibration depth and significance $\quad 90$

4.4.3.2.-Alkenone-based SST reconstruction $\quad 92$

4.4.4.-Total concentration of $\mathrm{C}_{37}$ alkenones and the $n$-hexacosan-1-ol index 93

4.4.5.-Spectral analysis 93

4.5.-DISCUSSION 93

4.5.1.-Stadials associated with Heinrich events 2 and 1 (H2 and $\mathrm{H} 1)$

4.5.2.-Last Glacial Maximum (LGM) 95

$\begin{array}{lr}\text { 4.5.3.-Termination 1a (T1a) } & 96\end{array}$

$\begin{array}{ll}\text { 4.5.4.-Bølling-Allerød (B-A) } & 98\end{array}$

4.5.5.-Younger Dryas (YD) 99

$\begin{array}{lr}\text { 4.5.6.-Termination } 1 \mathrm{~b}(\mathrm{~T} 1 \mathrm{~b}) & 100\end{array}$

$\begin{array}{lr}\text { 4.5.7.-Holocene } & 101\end{array}$

$\begin{array}{lr}\text { 4.5.8.-Periodicities } & 102\end{array}$

4.6.-CONCLUSIONS 103

$\begin{array}{ll}\text { Acknowledgements } & 104\end{array}$

$\begin{array}{ll}\text { Appendix A. Taxonomic appendix } & 104\end{array}$

$\begin{array}{ll}\text { References } & 105\end{array}$ 
CAPÍTULO 5.-ATMOSPHERIC PATTERNS DRIVING HOLOCENE PRODUCTIVITY IN THE ALBORAN SEA (WESTERN MEDITERRANEAN): A MULTIPROXY APPROACH

Resumen 116

$\begin{array}{ll}\text { Abstract } & 117\end{array}$

$\begin{array}{ll}\text { 5.1.-INTRODUCTION } & 118\end{array}$

5.2.-AREA OF STUDY: MODERN WATER MASSES AND CLIMATIC DYNAMICS

5.3.-MATERIALS AND METHODS 121

$\begin{array}{ll}\text { 5.3.1.-Age model } & 121\end{array}$

$\begin{array}{ll}\text { 5.3.2.-Coccolithophore analysis and taxonomy } & 122\end{array}$

$\begin{array}{lr}\text { 5.3.3.-Oxygen stable isotopes } & 123\end{array}$

$\begin{array}{lr}\text { 5.3.4.-Molecular biomarkers } & 123\end{array}$

$\begin{array}{ll}\text { 5.3.5.-Statistical approaches } & 124\end{array}$

$\begin{array}{ll}\text { 5.4.-RESULTS } & 124\end{array}$

$\begin{array}{ll}\text { 5.4.1.-Calcareous nannoflora distribution } & 124\end{array}$

$\begin{array}{ll}\text { 5.4.2.-Sea surface temperature (SST) } & 126\end{array}$

$\begin{array}{ll}\text { 5.4.3.-Oxygen isotopic record } & 127\end{array}$

5.4.4.-Total concentration of $\mathrm{C}_{37}$ alkenones and the $n$-hexacosan-1-ol index 127

$\begin{array}{ll}\text { 5.5.-DISCUSSION } & 127\end{array}$

$\begin{array}{ll}\text { 5.5.1.-General primary productivity and SST trends } & 127\end{array}$

$\begin{array}{lr}\text { 5.5.2.-Short-term changes } & 129\end{array}$

$\begin{array}{lr}\text { 5.5.2.1.-The } 7.7 \mathrm{ka} \text { event } & 129\end{array}$

5.5.2.2.-Variability of the productive "Malaga cell" 130

$\begin{array}{ll}\text { 5.5.3.-Proposed forced mechanisms } & 134\end{array}$

$\begin{array}{ll}\text { 5.6.-CONCLUSIONS } & 136\end{array}$

$\begin{array}{lr}\text { Acknowledgements } & 137\end{array}$

$\begin{array}{lr}\text { References } & 137\end{array}$

CAPÍTULO 6.-HIGH-RESOLUTION SEA SURFACE SALINITY RECONSTRUCTION FOR THE LAST 25 KYR IN THE WESTERN MEDITERRANEAN FROM A COCCOLITHOPHORE-BASED TRANSFER FUNCTION

$\begin{array}{ll}\text { Resumen } & 150\end{array}$

Abstract 151

6.1.-INTRODUCTION 152

6.2.-STUDY AREA AND OCEANOGRAPHIC SETTING 153

6.3.-MATERIALS AND METHODS 155

$\begin{array}{ll}\text { 6.3.1.-Modern training set } & 155\end{array}$

$\begin{array}{lr}\text { 6.3.1.1.-Surface sediment samples } & 155\end{array}$

$\begin{array}{lr}\text { 6.3.1.2.-Environmental variables } & 155\end{array}$

$\begin{array}{ll}\text { 6.3.2.-Fossil data set } & 155\end{array}$

$\begin{array}{ll}\text { 6.3.3.-Micropaleontological analyses } & 155\end{array}$

$\begin{array}{lr}\text { 6.3.4.-Statistical analyses } & 159\end{array}$

6.3.4.1.-Relationship between coccolithophore assemblages and environmental variables 159

$\begin{array}{ll}\text { 6.3.4.2.-Transfer function } & 160\end{array}$ 
$\begin{array}{lc}\text { 6.3.4.3.-SSS reconstruction and evaluation } & 161\end{array}$

$\begin{array}{ll}\text { 6.4.-RESULTS } & 161\end{array}$

6.4.1.-Geographical distribution of coccolithophores 162

6.4.2.-Relationship between coccolithophores and environmental variables 163

$\begin{array}{ll}\text { 6.4.3.-Transfer functions } & 163\end{array}$

$\begin{array}{lr}\text { 6.4.4.-SSS reconstruction } & 165\end{array}$

$\begin{array}{ll}\text { 6.5.-DISCUSSION } & 167\end{array}$

$\begin{array}{ll}\text { 6.5.1.-Geographic coccolithophore distribution and SSS } & 167\end{array}$

$\begin{array}{ll}\text { 6.5.2.-Transfer function quality } & 168\end{array}$

$\begin{array}{ll}\text { 6.5.3.-Downcore SSS reconstruction } & 169\end{array}$

6.5.4.-SSS changes in the Alboran Sea over the last 25,000 yr 170

6.5.4.1.-H2 and H1 171

$\begin{array}{ll}\text { 6.5.4.2.-Last Glacial Maximum (LGM) } & 171\end{array}$

$\begin{array}{ll}\text { 6.5.4.3.-Oldest Dryas } & 171\end{array}$

6.5.4.4.-Bølling-Allerød (B-A) 173

6.5.4.6.-Younger Dryas (YD) and the Holocene 174

$\begin{array}{ll}\text { 6.6.-CONCLUSIONS } & 176\end{array}$

$\begin{array}{ll}\text { Acknowledgements } & 176\end{array}$

$\begin{array}{ll}\text { References } & 177\end{array}$

\section{CAPÍTULO 7.-CONCLUSIONES}

7.1.-Variabilidad de la productividad de cocolitóforos y de la dinámica de la columna de agua durante los últimos 25 ka en el mar de Alborán

7.2.-Variabilidad de la productividad de cocolitóforos durante el Holoceno en el mar de Alborán

7.3.-Desarrollo de funciones de transferencia para el cálculo de la salinidad superficial marina a partir de cocolitóforos

ANEXOS

ANEXO I.-Lámina fotográfica

ANEXO II.-Procedimientos adoptados para los análisis micropaleontológicos $\quad 194$

ANEXO III.-Procedimientos adoptados para los análisis de isótopos de oxígeno 196

ANEXO IV.-Procedimientos adoptados para el análisis de compuestos orgánicos 198

$\begin{array}{ll}\text { ANEXO V.-Lista de acrónimos } & 201\end{array}$

ANEXO VI.-Índice general 203

$\begin{array}{lr}\text { Agradecimientos } & 205\end{array}$ 


\section{RESUMEN}

La presente tesis doctoral tiene como objetivo estudiar la variabilidad temporal de la productividad de cocolitóforos y la evolución climática en el mar Mediterráneo occidental durante los últimos 25 ka mediante el estudio micropaleontológico y geoquímico de registros sedimentarios marinos. Los cocolitóforos son un grupo de algas unicelulares marinas y conforman uno de los principales componentes del fitoplancton en los océanos actuales. Estos organismos secretan placas calcificadas (cocolitos) que, tras su sedimentación en los fondos marinos, pueden preservarse en el registro fósil. La abundancia y diversidad de los cocolitóforos están altamente influenciadas por las condiciones ambientales en las que proliferan, por lo que el estudio de los cocolitos preservados en el registro sedimentario aporta información útil para la realización de reconstrucciones paleoambientales.

En esta tesis doctoral se han analizado las asociaciones cocolitoforales de dos testigos sedimentarios pertenecientes al mar de Alborán: el testigo CEUTA10PC08 que abarca desde 25 hasta 4,5 ka, y el testigo HER-GC-T1 que abarca desde 23,5 hasta 0 ka. También se han analizado los registros de isótopos de oxígeno y temperatura superficial marina (TSM) de ambos testigos y algunos registros de marcadores orgánicos del testigo HER-GC-T1. El estudio combinado de todos los registros mencionados a alta resolución ha permitido reconstruir la variabilidad oceanográfica y climática del mar Mediterráneo occidental desde el Último Máximo Glacial (23 ka). También se han considerado las asociaciones cocolitoforales en un conjunto de muestras de sedimento superficial con el objetivo de explorar el potencial de los cocolitóforos para la reconstrucción cuantitativa de variables ambientales mediante el desarrollo de funciones de transferencia.

La productividad de cocolitóforos en el mar de Alborán está altamente influenciada por procesos climáticos tanto globales como locales desde el Último Máximo Glacial. En términos generales, se ha observado que la productividad ha sido mayor en el área más próxima al estrecho de Gibraltar, debido a la influencia del agua atlántica. Esta masa de agua resulta ser un mecanismo transmisor de la señal climática del Atlántico norte a la región Mediterránea. Sin embargo, a medida que el agua atlántica se mezcla con la mediterránea, los procesos locales y la configuración hidrográfica ejercen una mayor influencia sobre la productividad de los cocolitóforos. El estudio de los cambios observados en los registros de cocolitóforos, isótopos de oxígeno, TSM, etc., ha permitido contextualizar la variabilidad temporal de la productividad de cocolitóforos y las dinámicas de la columna de agua dentro de cambios climáticos globales conocidos como los estadiales asociados a los eventos Heinrich, el Bølling-Allerød o el Younger Dryas. 
Durante el Holoceno se ha observado que la productividad de los cocolitóforos en el testigo HER-GC-T1 está estrechamente ligada a otros procesos de origen físico, como la producción de Agua Mediterránea Profunda Occidental en el golfo de León y el control hidráulico que ejerce el estrecho de Gibraltar. Los patrones de circulación atmosférica son propuestos como responsables últimos de la variabilidad de la productividad de los cocolitóforos durante el Holoceno. A este respecto, se ha observado una relación entre los procesos físicos arriba mencionados y la Oscilación del Atlántico Norte, resultado que pone de manifiesto la teleconexión climática existente entre el Atlántico norte y el mar Mediterráneo occidental.

Por último, el estudio de la relación existente entre los censos de cocolitóforos en muestras de sedimento superficial procedentes del mar Mediterráneo occidental y las variables ambientales asociadas a su columna de agua ha permitido el desarrollo de una función de transferencia para la reconstrucción cuantitativa de la salinidad marina superficial (SMS) a alta resolución para los últimos $25 \mathrm{ka}$ en el mar de Alborán. La evaluación estadística del modelo de regresión y calibración y la comparación de la reconstrucción obtenida con otros registros de SMS localizados en la zona de estudio demuestran el elevado potencial de los cocolitóforos en funciones de transferencia para realizar reconstrucciones ambientales cuantitativas. La función de transferencia obtenida se propone como un nuevo indicador para reconstruir la variabilidad de la SMS a partir de asociaciones cocolitoforales fósiles de otros testigos sedimentarios del mar Mediterráneo occidental, especialmente para los últimos $15 \mathrm{ka}$. 


\section{ABSTRACT}

The presented thesis aims at studying the temporal variability of the coccolithophore productivity and the climatic evolution in the Western Mediterranean Sea for the last $25 \mathrm{kyr}$ through the micropaleontological and geochemical study of marine sediment records. Coccolithophores are marine unicellular algae and one of the main phytoplankton components in the current oceans. These organisms secrete calcified scales (coccoliths) that, after their sedimentation on the seafloor, may be preserved in the fossil record. Coccolithophore abundance and diversity are highly influenced by the environmental conditions that prevailed at time these organisms inhabited the ocean. Hence, the study of coccoliths preserved in the sedimentary record provides useful information to perform paleoenvironmental reconstructions.

In this thesis, the coccolithophore assemblages from two sediment cores from the Alboran Sea have been analyzed: the CEUTA10PC08 core, which covers from 25 to $4.5 \mathrm{ka}$, and the HER-GC-T1 core, which covers from 23.5 to $0 \mathrm{ka}$. Along with these data, oxygen isotopes and sea surface temperature (SST) from both cores, together with several organic biomarkers from HER-GC-T1 core have also been analysed. The combined study at high-resolution of all these records has allowed reconstructing the oceanographic and climatic variability of the Western Mediterranean since the Last Glacial Maximum (23 ka). The coccolithophore assemblages from a set of sea surface sediment samples have also been studied in order to explore the potential of coccolithophores to quantitatively reconstruct environmental variables by developing transfer functions.

Coccolithophore productivity in the Alboran Sea has proved to be highly influenced by global and local climatic processes since the Last Glacial Maximum. In general terms, it has been observed that productivity was higher in the area closer to the Strait of Gibraltar, due to the influence of the inflow of Atlantic waters. This water mass turned to be a transmitting mechanism for the North Atlantic climatic signal to the Mediterranean region. Nevertheless, other local processes and hydrographic configuration exert a major influence on coccolithophore productivity in more distant areas. The study of observed changes in the coccolithophore records, oxygen isotopes, SST, etc., has allowed to relate the temporal variability of coccolithophore productivity and the water column dynamics to the well-known global climate changes such as the stadials associated to the Heinrich Events, the BøllingAllerød or the Younger Dryas.

During the Holocene, it was observed that coccolithophore productivity in HER-GC-T1 core location is closely related to other processes of physical origin, like the Western Mediterranean Deep Water formation in the Gulf of Lions or the hydraulic control exerted by the Strait of Gibraltar. Atmospheric circulation patterns are proposed to be responsible for variations in 
Holocene coccolithophore productivity. In this regard, a relationship between the aforementioned physical processes and the North Atlantic Oscillation has been observed. These results highlight the climatic teleconnection that exists between the North Atlantic and the Western Mediterranean regions.

Finally, the study of the current relationship between coccolithophore census counts from surface sediment samples from the Western Mediterranean Sea and the associated environmental variables has allowed the development of a transfer function to quantitatively reconstruct sea surface salinity (SSS) at high resolution for the last $25 \mathrm{kyr}$ in the Alboran Sea. The statistical assessment of the regression and calibration model and the comparison of the obtained reconstructions with other SSS records from nearby areas show good results suggesting that coccolithophores have a great potential in transfer functions to perform quantitative environmental reconstructions. The obtained transfer function is proposed as a new proxy to reconstruct SSS variability from coccolithophore census counts from other sediment cores in the Western Mediterranean Sea, especially for the last $15 \mathrm{kyr}^{1}$.

\footnotetext{
${ }^{1}$ In this thesis geological date will be expressed in "annus" (a) and geohistorical duration will be expressed in "years" (yr), and their related multiples, according to Aubry et al. (2009).
} 


\section{INTRODUCCIÓN Y JUSTIFICACIÓN}

El estudio de la variabilidad climática desde el Último Máximo Glacial ha cobrado especial relevancia a lo largo de las dos últimas décadas debido a que es el periodo más reciente, por lo que el conocimiento en detalle de la variabilidad del sistema natural durante el mismo puede ser de gran utilidad a la hora de predecir posibles escenarios climáticos futuros (IPCC, 2013). Sin embargo, no basta únicamente con conocer dicha variabilidad. Es necesario identificar sus causas y comprender los mecanismos de teleconexión que las transmiten de unas regiones a otras.

El mar Mediterráneo occidental es una cuenca situada en latitudes medias, semi-aislada y conectada con el océano Atlántico únicamente a través del estrecho de Gibraltar. En esta cuenca las señales climáticas y oceanográficas se amplifican (Cacho et al., 2002) y son registradas en los sedimentos marinos a más resolución que en océano abierto, donde se atenúan más fácilmente (Thunell y Williams, 1989). Por este motivo, el mar Mediterráneo occidental es una zona idónea para el estudio a alta resolución de las variaciones climáticas y oceanográficas que lo caracterizaron en el pasado, así como de los procesos de teleconexión entre latitudes medias y altas. Diversos aspectos de la variabilidad climática y oceanográfica en esta región durante los últimos $25 \mathrm{ka}$ han sido ampliamente estudiados por varios autores (Cacho et al., 2001; JiménezEspejo et al., 2007; Frigola et al., 2008; Combourieu Nebout et al., 2009; Fletcher et al., 2010; Rodrigo-Gámiz et al., 2011). Sin embargo, y a pesar de la existencia de estudios previos sobre su productividad primaria (Bárcena et al., 2001; Colmenero-Hidalgo et al., 2004) aún se disponen de pocas evidencias sobre la variabilidad de ésta a escala secular y los posibles procesos que la controlan (Abrantes et al., 2012).

Los cocolitóforos son algas unicelulares marinas y uno de los principales componentes del fitoplancton marino. Estos organismos producen placas calcificadas (cocolitos) que sedimentan en los fondos oceánicos pudiendo llegar a preservarse en el registro fósil. El estudio de las asociaciones de cocolitóforos y su abundancia en los sedimentos marinos proporciona una valiosa información acerca de la productividad primaria de la región a la que pertenecen, ya que estos organismos responden rápidamente a la disponibilidad de nutrientes. El estudio de estos registros en combinación con otros indicadores paleoambientales es de gran utilidad para evaluar las condiciones ambientales en las que vivieron estas algas e identificar los procesos que controlaron y/o influenciaron su productividad.

Los cocolitóforos son ampliamente utilizados para realizar reconstrucciones paleoceanográficas cualitativas. Sin embargo, y a diferencia de otros organismos marinos como los foraminíferos, las diatomeas, los radiolarios o los dinoflagelados, los cocolitóforos no se 
utilizan de forma sistemática para el desarrollo de funciones de transferencia y la obtención de registros cuantitativos de las variables ambientales. Surge así, por tanto, la necesidad de explorar la adecuación de estos organismos para el desarrollo y aplicación de funciones de transferencia con el fin de reconstruir de forma cuantitativa la variabilidad de ciertos parámetros ambientales en el pasado.

Esta tesis doctoral se centra en el estudio a alta resolución de las asociaciones cocolitoforales del mar Mediterráneo occidental en combinación con registros de isótopos de oxígeno, temperatura superficial marina y marcadores orgánicos durante los últimos 25 ka con el fin de describir su productividad y reconstruir la variabilidad climática y oceanográfica de esta región a escala secular así como identificar posibles procesos de teleconexión con el Atlántico norte.

\section{HIPÓTESIS DE TRABAJO}

Los cocolitos preservados en los sedimentos oceánicos proporcionan información acerca de la productividad primaria de la región y de las condiciones ambientales en las que vivieron estos organismos. El estudio combinado de estos registros con los de otros indicadores paleoambientales es de gran utilidad para la evaluación de la variabilidad climática y oceanográfica que controla o influye sobre la productividad de los cocolitóforos.

\section{OBJETIVOS}

\section{Objetivos generales:}

1.-Obtener registros a alta resolución de la productividad de cocolitóforos, de temperatura superficial marina e isótopos de oxígeno y de biomarcadores orgánicos del mar Mediterráneo occidental a lo largo de la última deglaciación.

2.-Identificar cambios regionales a diversas escalas temporales para reconstruir la variabilidad oceanográfica y ambiental del mar Mediterráneo occidental desde la última deglaciación.

3.-Contextualizar dicha variabilidad dentro de cambios globales conocidos para comprender posibles procesos de teleconexión entre latitudes medias y altas.

4.-Explorar el potencial de los cocolitóforos para el desarrollo de funciones de transferencia que permitan reconstruir variables ambientales desde el último Último Máximo Glacial hasta el presente.

\section{Objetivos específicos:}

1.1.-Caracterizar las asociaciones de cocolitóforos de los dos testigos sedimentarios del mar de Alborán (Mediterráneo occidental). 
2.1.-Identificar, a diferentes escalas temporales, los cambios acontecidos en la productividad, la temperatura superficial marina, los isótopos de oxígeno y los biomarcadores orgánicos del mar Mediterráneo occidental.

2.2.-Reconstruir posibles escenarios paleoceanográficos y paleoclimáticos que expliquen la variabilidad encontrada.

3.1.-Comparar los registros obtenidos en el objetivo general 1 con otros de diversa naturaleza procedentes de otras zonas del mar Mediterráneo y del océano Atlántico norte.

3.2.-Identificar y describir los posibles patrones de teleconexión entre las regiones del Mediterráneo y del Atlántico norte.

4.1.-Caracterizar las asociaciones de cocolitóforos de las muestras de sedimento superficial.

4.2.-Evaluar las técnicas estadísticas necesarias para el desarrollo de una función de transferencia.

4.3.-Aplicar la función de transferencia obtenida al registro sedimentario fósil para la reconstrucción de variables ambientales.

\section{ESTRUCTURA DE LA TESIS DOCTORAL:}

La presente tesis doctoral se divide en siete capítulos y seis anexos.

En el Capítulo 1 se realiza una introducción a las dinámicas oceánica y atmosférica de la zona de estudio y se explican los aspectos más relevantes de las mismas. Se describen la circulación superficial, intermedia y profunda del mar Mediterráneo occidental. También se explican las principales características del mar de Alborán y los aspectos más importantes relativos a su productividad primaria y a la dinámica atmosférica. Por último se abordan los procesos de teleconexión con el océano Atlántico norte más relevantes para el desarrollo de esta tesis doctoral.

En el Capítulo 2 se realiza una introducción a los cocolitóforos, principal objeto de estudio en esta tesis doctoral. En el mismo se explican y describen sus principales características, tipos y ciclos de vida, así como la ecología, distribución espacial y sistemática de las especies consideras.

En el Capítulo 3 de describen los materiales empleados y las técnicas y métodos aplicados. En el mismo se detallan las características geológicas e información relativa a los dos testigos sedimentarios estudiados, así como su cronoestratigrafía. También se detalla la información relativa a las muestras de sedimento superficial y los datos de variables ambientales utilizados en la creación de la base de datos para el desarrollo de la función de transferencia. Respecto a las técnicas y métodos, se describen de forma general los empleados en los análisis 
micropaleontológicos de las asociaciones cocolitoforales, en los análisis de isótopos de oxígeno, de alquenonas y de biomarcadores orgánicos, y en el tratamiento estadístico de los datos. Este capítulo se complementa con información adicional relativa a los protocolos empleados y que han sido considerados en los anexos.

El Capítulo 4 lo compone el primer artículo científico del que la doctoranda es la autora principal. En el mismo se abordan los objetivos generales 1 y 2 mediante el estudio a alta resolución de las asociaciones cocolitoforales pertenecientes a dos testigos sedimentarios del mar de Alborán y su combinación con los registros de isótopos de oxígeno, TSM y biomarcadores orgánicos durante los últimos $25 \mathrm{ka}$.

* Ausín B, Flores JA, Bárcena MA, Sierro FJ, Francés G, Gutiérrez-Arnillas E, Hernández-Almeida I, Martrat B, Grimalt JO y Cacho I, (2015) Coccolithophore productivity and surface water dynamics in the Alboran Sea during the last $25 \mathrm{kyr}$. Palaeogeography, Palaeoclimatology, Palaeoecology 418: 126-140. DOI: 10.1016/j.palaeo.2014.11.011

El Capítulo 5 lo compone el segundo artículo científico del que la doctoranda es la autora principal. En el mismo se abordan los objetivos generales 1,2 y 3 mediante el estudio de las asociaciones cocolitoforales de un testigo sedimentario del mar de Alborán, los registros de isótopos de oxígeno, TSM y biomarcadores orgánicos pertenecientes al mismo testigo y su comparación con indicadores paleoambientales pertenecientes a testigos de la misma cuenca durante el Holoceno.

* Ausín B, Flores JA, Sierro FJ, Cacho I, Hernández-Almeida I, Martrat B y Grimalt JO. (2015) Atmospheric patterns driving Holocene productivity in the Alboran Sea (Western Mediterranean): A multiproxy approach. The Holocene 25: 583-595. DOI: $10.1177 / 0959683614565952$

El Capítulo 6 lo compone el tercer artículo científico del que la doctoranda es la autora principal. En el mismo se abordan los objetivos generales 2, 3 y 4 mediante el estudio de las asociaciones cocolitoforales pertenecientes a varias muestras de sedimento, el desarrollo de una función de transferencia y su aplicación a un testigo sedimentario del mar de Alborán para reconstruir la salinidad marina superficial y describir su variabilidad a escala secular durante los últimos $25 \mathrm{ka}$.

* Ausín B, Hernández-Almeida I, Flores JA, Sierro FJ, Grosjean M, Francés G, Alonso B. High-resolution sea surface salinity reconstruction for the last $25 \mathrm{kyr}$ in the Western Mediterranean from a coccolithophore-based Transfer Function. Enviado para publicación el 25/02/2015 a Quaternary Science Reviews. En revisión.

El Capítulo 7 resume las principales conclusiones derivadas de esta tesis doctoral. 
Finalmente, se presentan una serie de anexos. El Anexo I recoge las imágenes de los cocolitos de los taxones más representativos contemplados en esta tesis bajo el microscopio óptico. El Anexo II presenta el procedimiento detallado adoptado para la preparación de las muestras para el análisis micropaleontológico de las asociaciones cocolitoforales. El Anexo III presenta el protocolo considerado para el análisis de isótopos estables de oxígeno. Por último, el Anexo IV presenta el protocolo seguido para el análisis de biomarcadores orgánicos que también incluyen las alquenonas. El Anexo $\mathbf{V}$ presenta un listado de los acrónimos utilizados en este manuscrito. El Anexo VI presenta un índice general de los términos más relevantes y las páginas en las que se tratan más a fondo.

\section{Referencias}

Aubry M-P, Van Couvering JA, Christie-Blick N, Landing E, Pratt BR, Owen DE, FerrusquíaVillafranca I, 2009, Terminology of Geological Time: Establishment of a Community Standard, Stratigraphy 6: 100-105.

Abrantes F, Voelker A, Sierro FJ, Naughton F, Rodrigues T, Cacho I, Ariztegui D, Brayshaw D, Sicre M-A, Batista L y Lionello P. (2012) 1- Paleoclimate Variability in the Mediterranean Region. The Climate of the Mediterranean Region. Oxford: Elsevier, 186.

Bárcena MA, Cacho I, Abrantes F, Sierro FJ, Grimalt JO y Flores JA. (2001) Paleoproductivity variations related to climatic conditions in the Alboran Sea (western Mediterranean) during the last glacial-interglacial transition: the diatom record. Palaeogeography, Palaeoclimatology, Palaeoecology 167: 337-357.

Cacho I, Grimalt JO y Canals M. (2002) Response of the Western Mediterranean Sea to rapid climatic variability during the last 50,000 years: a molecular biomarker approach. Journal of Marine Systems 33-34: 253-272.

Cacho I, Grimalt JO, Canals M, Sbaffi L, Shackleton NJ, Schönfeld J y Zahn R. (2001) Variability of the western Mediterranean Sea surface temperature during the last 25,000 years and its connection with the Northern Hemisphere climatic changes. Paleoceanography 16: 40-52.

Colmenero-Hidalgo E, Flores JA, Sierro FJ, Bárcena MÁ, Löwemark L, Schönfeld J y Grimalt JO. (2004) Ocean surface water response to short-term climate changes revealed by coccolithophores from the Gulf of Cadiz (NE Atlantic) and Alboran Sea (W Mediterranean). Palaeogeography, Palaeoclimatology, Palaeoecology 205: 317-336.

Combourieu Nebout N, Peyron O, Dormoy I, Desprat S, Beaudouin C, Kotthoff U y Marret F. (2009) Rapid climatic variability in the west Mediterranean during the last 25000 years from high resolution pollen data. Climate of the Past 5: 503-521. 
Fletcher WJ, Sánchez Goñi MF, Peyron O y Dormoy I. (2010) Abrupt climate changes of the last deglaciation detected in a Western Mediterranean forest record. Climate of the Past 6: 245-264.

Frigola J, Moreno A, Cacho I, Canals M, Sierro FJ, Flores JA y Grimalt JO. (2008) Evidence of abrupt changes in Western Mediterranean Deep Water circulation during the last 50 kyr: A high-resolution marine record from the Balearic Sea. Quaternary International 181: 88-104.

IPCC. (2013) Climate Change 2013: The Physical Science Basis. Contribution of Working Group I to the Fifth Assessment Report of the Intergovernmental Panel on Climate Change En: Stocker TF, D. Qin, GK. Plattner, M. Tignor, SK. Allen, J. Boschung, A. Nauels, Y. Xia, V. Bex y PM. Midgley (ed) Cambridge University Press. Cambridge, United Kingdom and New York, NY, USA, 1535 pp.

Jiménez-Espejo FJ, Martínez-Ruiz F, Sakamoto T, Lijima K, Gallego-Torres D y Harada N. (2007) Paleoenvironmental changes in the western Mediterranean since the last glacial maximum: High resolution multiproxy record from the Algero-Balearic basin. Palaeogeography, Palaeoclimatology, Palaeoecology 246: 292-306.

Rodrigo-Gámiz M, Martínez-Ruiz F, Jiménez-Espejo FJ, Gallego-Torres D, Nieto-Moreno V, Romero O y Ariztegui D. (2011) Impact of climate variability in the western Mediterranean during the last 20,000 years: oceanic and atmospheric responses. Quaternary Science Reviews 30: 2018-2034.

Thunell RC y Williams DF. (1989) Glacial-Holocene salinity changes in the Mediterranean Sea: hydrographic and depositional effects. Nature 338: 493-496. 


\section{CAPÍTULO 1}

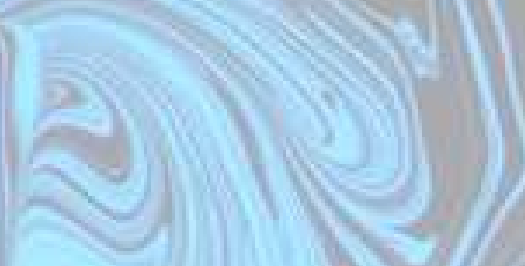






\section{1.-DINÁMINA OCEÁNICA Y ATMOSFÉRICA DE LA ZONA DE ESTUDIO}

\section{1.-EL MAR MEDITERRÁNEO}

El interés humano y científico en el clima del mar Mediterráneo está motivado por la convergencia en esta región de varios factores poblacionales, sociales, económicos y ambientales. El mar Mediterráneo baña las costas de 21 países pertenecientes a África, Asia y Europa, que cuentan con aproximadamente 400 millones de habitantes, de los cuales cerca de 145 millones habitan sus zonas costeras. Densamente poblada y sometida a un gran estrés ambiental, la región mediterránea es potencialmente sensible al cambio climático.

Sin embargo, el mar Mediterráneo también desempeña un papel relevante dentro del sistema climático global, constituyendo una fuente de humedad y un reservorio de calor para las zonas continentales que lo rodean y siendo el origen de gran parte del agua salina que circula por los niveles intermedios del océano Atlántico (Lionello et al., 2006).

El mar Mediterráneo (Figura 1.1) está limitado por Europa al norte, Asia al este y África al sur. Se localiza en latitudes medias, entre $\operatorname{los} 30^{\circ}$ y los $45^{\circ}$ norte y $\operatorname{los}-5^{\circ}$ y los $35^{\circ}$ de longitud este. Su superficie abarca $2,5 \mathrm{~km}^{2}$, extendiéndose $3.700 \mathrm{~km}$ longitudinalmente y $1600 \mathrm{~km}$ latitudinalmente. Su profundidad media es de $1.500 \mathrm{~m}$ alcanzando una profundidad máxima de $5.150 \mathrm{~m}$ en el mar Jónico. Es una cuenca parcialmente aislada, conectada con el océano abierto únicamente a través del estrecho de Gibraltar. Cuenta con un balance hídrico negativo (Bèthoux, 1979), lo que significa que la evaporación de agua superficial a la atmósfera excede a la suma de la precipitación y los aportes fluviales. La pérdida evaporativa oscila entre 500 y $700 \mathrm{~mm}$ anuales (Mariotti et al., 2002) y es compensada por la entrada de un flujo neto de agua atlántica $\left(500 \mathrm{~mm} \mathrm{a}^{-1}\right.$ ) restringido por el estrecho de Gibraltar (Bethoux, 1980; Fenoglio-Marc et al., 2013), de manera que las variaciones globales del nivel del mar ejercen un control adicional sobre la hidrografía del Mediterráneo (Bryden y Kinder, 1991). Al tratarse de una cuenca de concentración, las señales climáticas y oceanográficas se amplifican (Cacho et al., 2002) y pueden estudiarse en los sedimentos marinos del mar Mediterráneo a mayor resolución que en océano abierto (Thunell y Williams, 1989).

En resumen, su localización en latitudes medias y sus peculiaridades físicas, morfológicas e hidrológicas, hacen del mar Mediterráneo una zona idónea para el estudio a alta resolución de las variaciones climáticas y oceanográficas que lo caracterizaron en el pasado, así como de los procesos climáticos de teleconexión entre latitudes medias y altas.

La presencia de golfos, islas y penínsulas dota al mar Mediterráneo de una compleja morfología, posibilitando su subdivisión en dos subcuencas principales: la occidental y la oriental (Figura 1.1). 


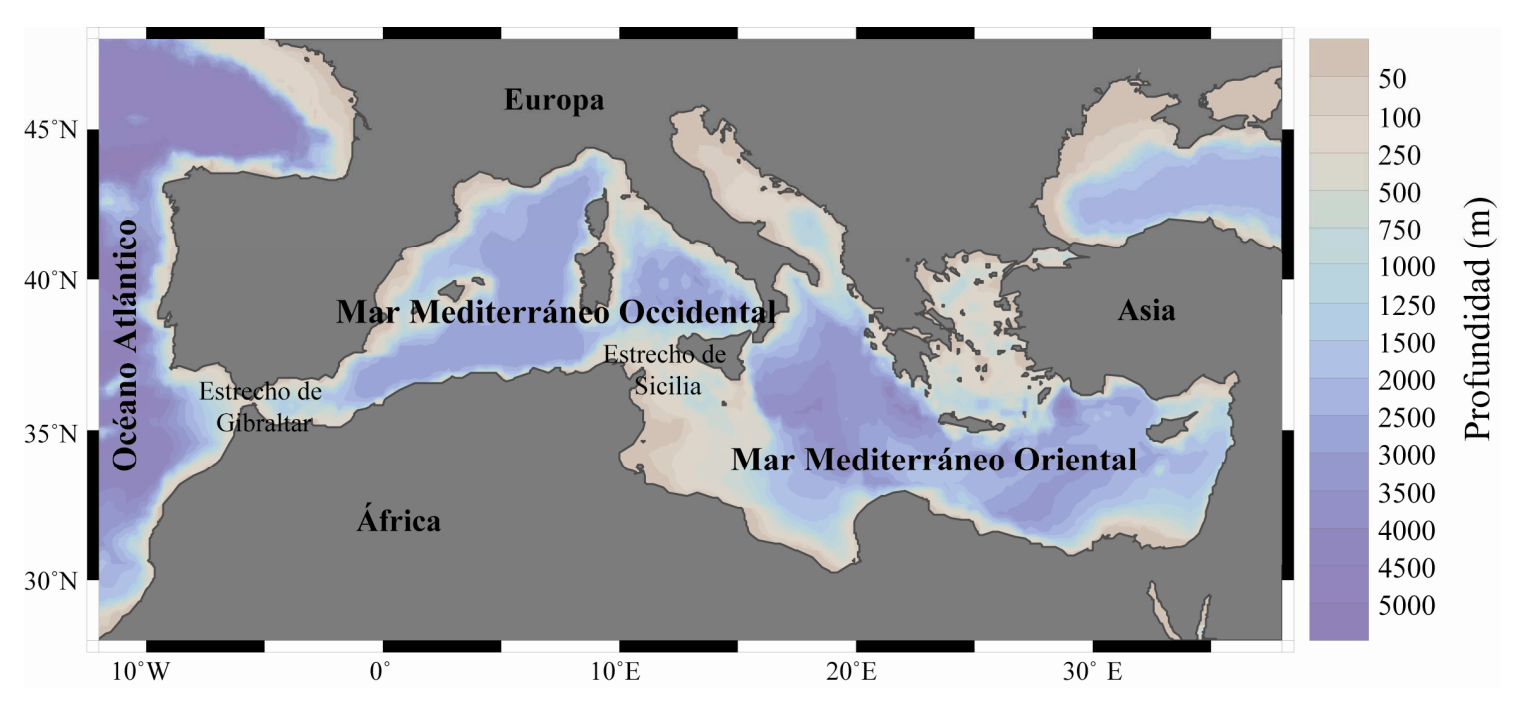

Figura 1.1. Mapa del mar Mediterráneo y sus dos subcuencas principales.

\section{2.-EL MAR MEDITERRÁNEO OCCIDENTAL}

El mar Mediterráneo occidental (Figura 1.2a) está delimitado al este por el estrecho de Sicilia de $400 \mathrm{~m}$ de profundidad y al oeste por el estrecho de Gibraltar. Este último abarca un sistema de umbrales y estrechos confinados en 14,5 km de ancho y $290 \mathrm{~m}$ de profundidad, jugando así un papel determinante en la conexión entre el mar Mediterráneo y el océano Atlántico.

El Mediterráneo occidental puede dividirse en otras subcuencas de menor tamaño. De este a oeste y de norte a sur: el mar Tirreno, la cuenca Liguro-Provenzal, el mar Catalano-Balear, la cuenca Argelina y el mar de Alborán (Figura 1.2a). Este último cobrará especial relevancia en el desarrollo de esta memoria.

\subsection{1.-Dinámica oceánica actual}

\subsubsection{1.-Circulación superficial}

Desde los proyectos pioneros "Dónde va?" (The Donde Va Group, 1984) y "The Western Mediterranean Circulation Experiment" (La Violette, 1990) la circulación oceánica del mar Mediterráneo occidental ha sido objeto de numerosas investigaciones. Debido al signo negativo de su balance hídrico presenta una circulación superficial de carácter anti-estuarino, conformando así una zona transicional donde el Agua Atlántica (Atlantic Water [AW]) entra en superficie en forma de chorro conocido como chorro Atlántico (Atlantic Jet [AJ]) (GarcíaLafuente et al., 1998). El AJ² describe dos giros anticiclónicos en el mar de Alborán: los giros

\footnotetext{
${ }^{2}$ Se utilizarán los acrónimos de la nomenclatura anglosajona por razones de consistencia con el resto del texto.
} 
occidental y oriental (Western Anticyclonic Gyre [WAG] y Eastern Anticyclonic Gyre [EAG] respectivamente) caracterizados por una alta variabilidad temporal (Cheney y Doblar, 1982).

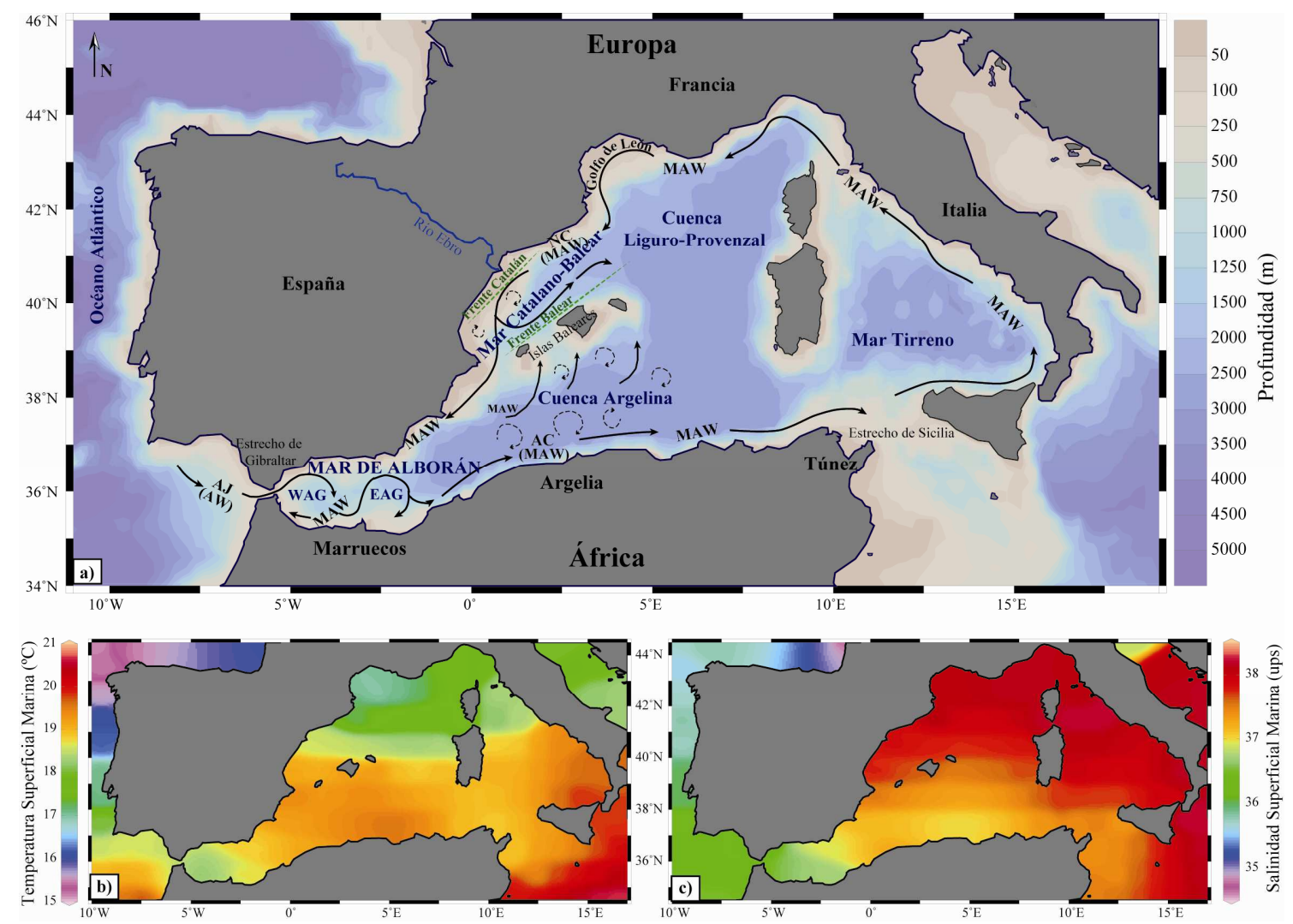

Figura 1.2. Mapa del mar Mediterráneo occidental. a) Patrón esquemático de circulación superficial. Distribución espacial del promedio anual desde 1995 hasta 2012 de: b) temperatura superficial marina $\left({ }^{\circ} \mathrm{C}\right)$ (Locarnini et al., 2010) y c) salinidad superficial marina (ups) (Zweng et al., 2013). Datos extraídos del World Ocean Atlas 2013 (WOA 13) y representados con el software Ocean Data View (Schlitzer, 2014). Leyenda: AJ: Atlantic Jet, AW: Atlantic Water, MAW: Modified Atlantic Water, AC: Algerian Current y NC: Northern Current.

A medida que el AJ discurre hacia el este, la AW se mezcla con el agua superficial mediterránea que encuentra a su paso, formando el Agua Atlántica Modificada (Modified Atlantic Water [MAW]) y ocupando los primeros 100-200 m de la columna de agua de todo el mar Mediterráneo (Millot, 1999). En el estrecho de Gibraltar la MAW se caracteriza por una salinidad media de 36,5 unidades prácticas de salinidad (ups) y una temperatura de $18,5{ }^{\circ} \mathrm{C}$ mientras que al norte del mar Mediterráneo occidental alcanza 38,2 ups y $17^{\circ} \mathrm{C}$ (Figura 1.2.b, c). Sin embargo, tanto los valores absolutos como la distribución espacial de propiedades como la temperatura pueden estacionalmente (Figura 1.3). El efecto de la estacionalidad afecta a la distribución espacial y los valores absolutos de otras variables ambientales como el oxígeno o los nutrientes. Por el contrario, la salinidad no presenta variaciones estacionales notables (Figura $1.3)$. 


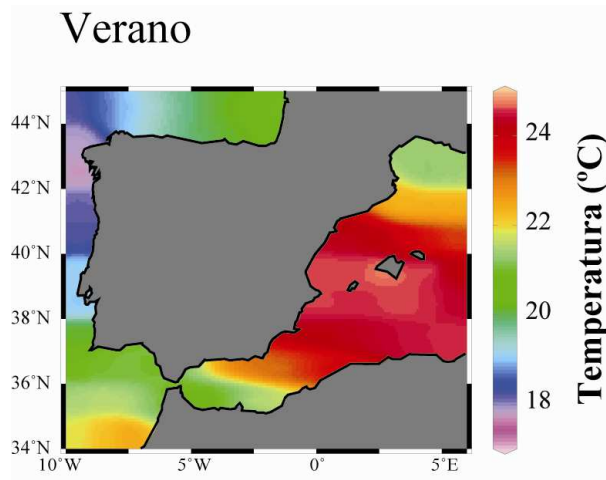

\section{Invierno}
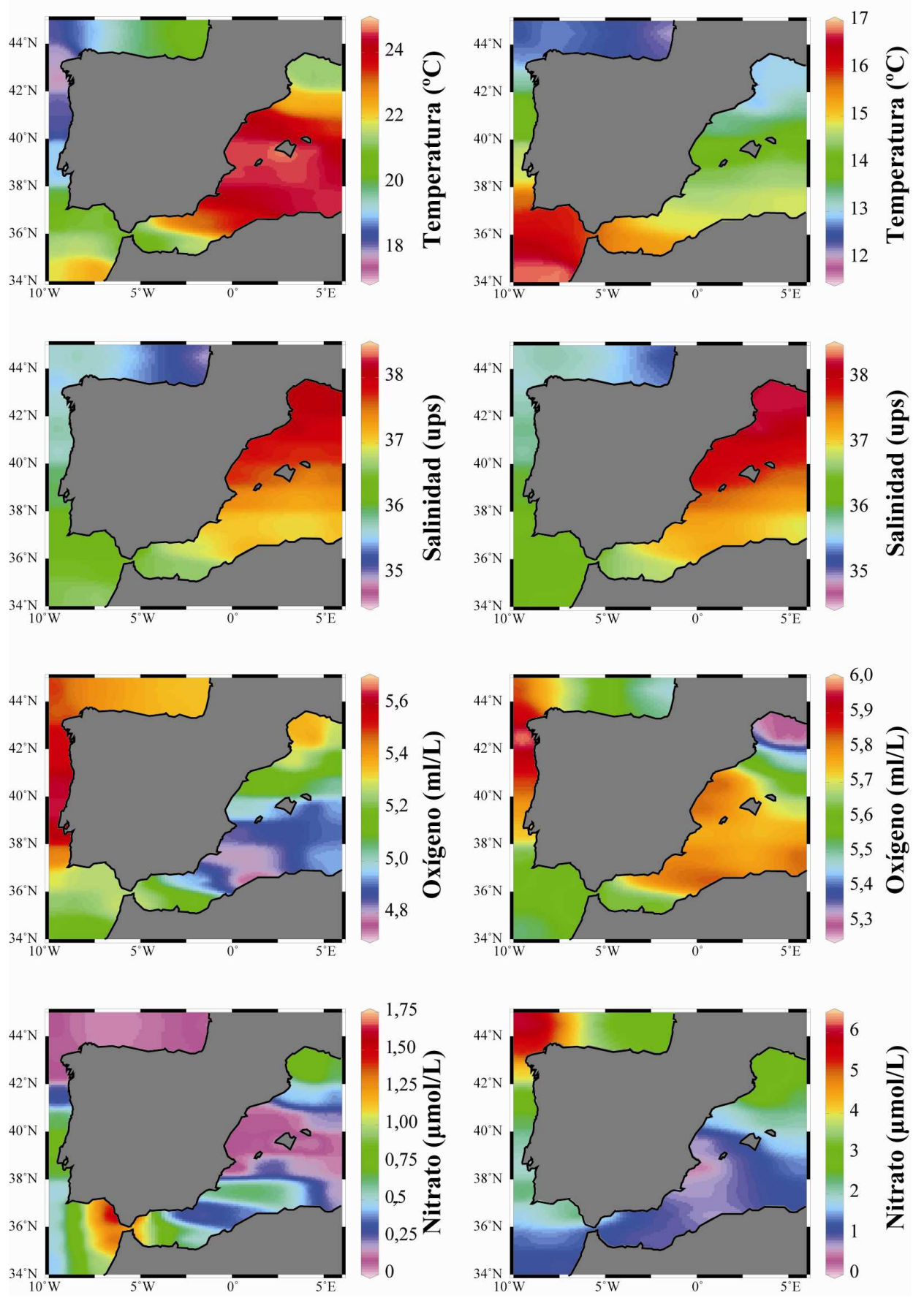

Figura 1.3. Variación estacional de la temperatura $\left({ }^{\circ} \mathrm{C}\right)$, la salinidad (ups), el oxígeno $(\mathrm{mL} / \mathrm{L})$ y los nitratos $(\mu \mathrm{mol} / \mathrm{L}$ ) a $10 \mathrm{~m}$ de profundidad entre el verano (julio-septiembre) y el invierno (junio-marzo) en el Mediterráneo occidental. Datos promediados entre 1995 y 2012 extraídos del World Ocean Atlas 2013 (WOA 13) y graficados con el software Ocean Data View (Schlitzer, 2014).

La MAW fluye hacia el este a lo largo del margen continental norteafricano formando la Corriente Argelina (Algerian Current [AC]) (Millot, 1985). Esta corriente genera meandros y remolinos de varios kilómetros de diámetro o "eddies", dando lugar a una de las regiones más energéticas de todo el mar Mediterráneo (Millot, 1999). Parte del volumen de la MAW deja al 
este el estrecho de Sicilia y continúa circulando por el mar Tirreno a lo largo de la costa occidental de la península italiana.

En su camino, la MAW ve aumentada su salinidad y su temperatura (Figura 1.2.b, c) y fluye como la "Corriente del Norte" (Northern Current $[\mathrm{NC}]$ ) por la cuenca Liguro-Provenzal hacia el mar Catalano-Balear (Millot, 1992). En esta región la morfología de los taludes continentales modifica la dirección de la NC que dibuja pequeños eddies anticiclónicos a su paso. El frente Catalán al norte y el frente Balear al sur delimitan la NC, separando sus aguas cálidas y salinas de los aportes fluviales del río Ebro y de la MAW más reciente que circunda el margen sur de las islas Baleares (Estrada y Margalef, 1988; Font et al., 1988). La NC entra en el mar de Alborán donde se encuentra con el flujo energético de la MAW más reciente, desviándose finalmente hacia la costa de Argelia (Millot, 1999).

\subsubsection{2.-Circulación en profundidad}

La circulación en profundidad en el mar Mediterráneo occidental puede simplificarse mediante un modelo de tres capas que considera las masas de agua más características de esta cuenca y con mayor relevancia para la realización del presente estudio (Figura 1.4).

Bajo la capa más superficial ocupada por la MAW fluye el Agua Intermedia Levantina (Levantine Intermediate Water [LIW]) de este a oeste hacia el estrecho de Gibraltar a 200-600 $\mathrm{m}$ de profundidad.

La tercera capa y más profunda (600-3000 m) la ocupa el Agua Mediterránea Profunda Occidental (Western Mediterranean Deep Water [WMDW]) que fluye junto a LIW hacia el estrecho de Gibraltar (Parrilla et al., 1986) (Figura 1.4).

La LIW y la WMDW tienen un origen termohalino, ya que se producen por convección a consecuencia de diferencias de temperatura y salinidad de las masas de agua involucradas en su formación. La LIW se forma en la cuenca oriental, frente a la costa turca meridional, en el giro de Rodas (Lascaratos et al., 1993). En esta zona la tasa de evaporación es muy elevada, dando

lugar a aguas muy salinas (>38,5 ups) que se hunden y fluyen hacia la cuenca occidental a través del canal de Sicilia (Figura 1.4). La WMDW se forma en mar abierto frente a las costas del golfo del León (Figura 1.2a) (MEDOCGROUP, 1970). La acción continua de los vientos Tramontana (componente norte) y Mistral (componente noroeste) sobre dicha región induce un flujo de calor y humedad desde la superficie oceánica hacia la atmósfera que precondiciona la inestabilidad de las masas de agua superficiales (Mertens y Schott, 1998; Rixen et al., 2005; Font et al., 2007; Smith et al., 2008). Estos vientos fríos y secos producen la evaporación de la MAW, que se vuelve más densa y finalmente se hunde formando la WMDW. Esta masa de agua discurre en profundidad hacia el estrecho de Gibraltar, en sentido opuesto a la MAW (Millot, 

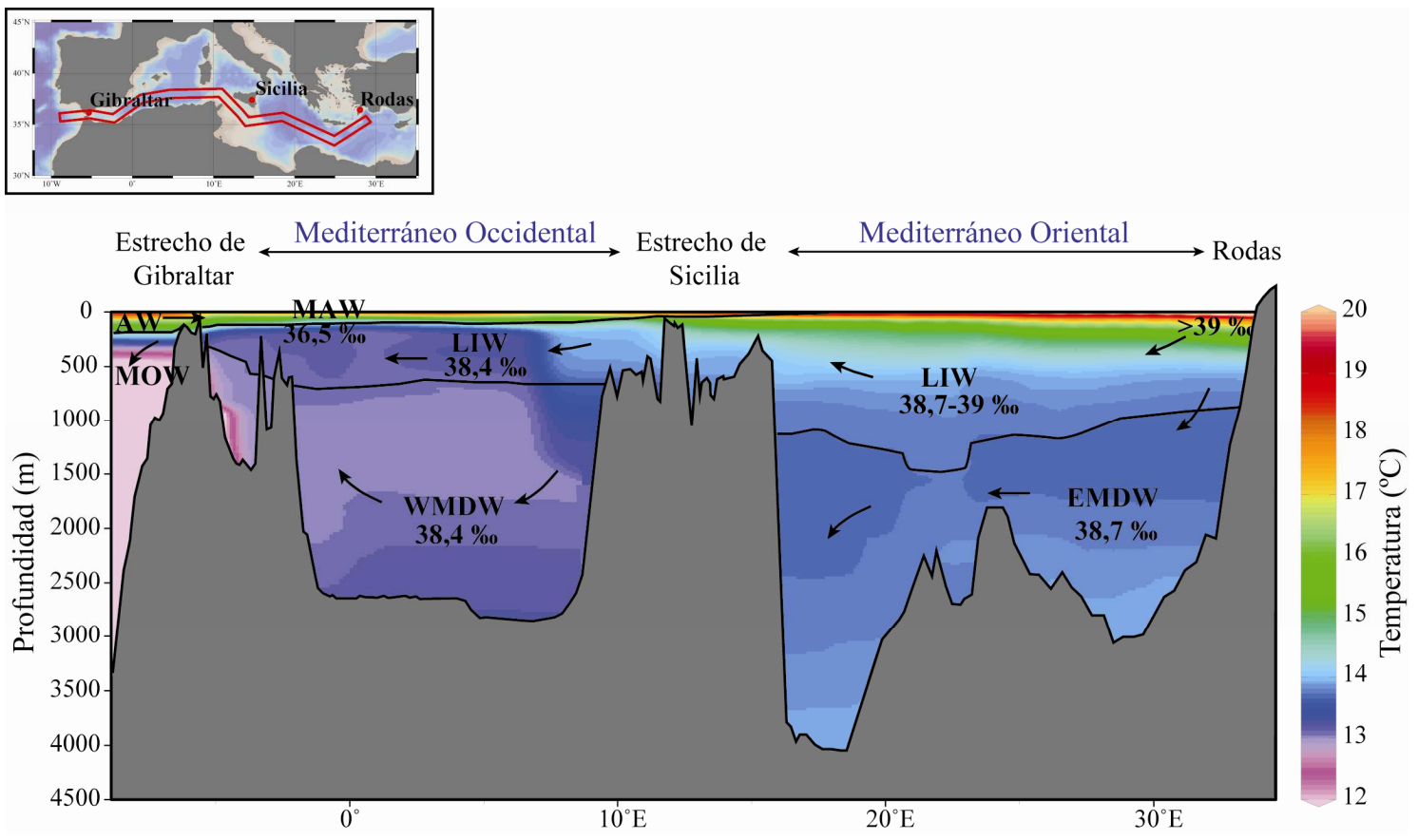

Figura 1.4. Esquema de la circulación profunda de las principales masas de agua del mar Mediterráneo y sus valores de salinidad y temperatura medios anuales promediados desde 1995 hasta 2012, correspondiente a la sección marcada con líneas rojas en el mapa superior de la figura. Modificada de Cramp y O'Sullivan (1999). Datos extraídos del WOA13 (Locarnini et al., 2013). Leyenda: AW: Atlantic Water, MOW: Mediterranean Outflowing Water, LIW: Levantine Intermediate Water, WMDW: Western Mediterranean Deep Water, EMDW: Eastern Mediterranean Deep Water. Modificada de (Cramp y O'Sullivan, 1999).

Tanto la LIW como la WMDW fluyen hacia el Atlántico en profundidad saliendo por el estrecho de Gibraltar como una nueva masa de agua cálida y salina llamada Flujo de Salida Mediterráneo (Mediterranean Outflowing Water [MOW]). Una vez en el Atlántico, la MOW discurre como una lengua salina a 600-2.500 $\mathrm{m}$ de profundidad hacia el oeste y hacia el norte, contribuyendo a la formación del Agua Profunda del Atlántico Norte (North Atlantic Deep Water [NADW]).

\section{3.-EL MAR DE ALBORÁN}

\subsection{1.-Hidrografía del mar de Alborán}

El mar de Alborán es la subcuenca más occidental del mar Mediterráneo, donde la confluencia del AJ con los giros WAG y EAG forma un patrón de circulación superficial que constituye una de sus muchas particularidades (Figura 1.5). Este sistema no es permanente, ya que los giros pueden variar tanto espacial como temporalmente, si bien es cierto que el WAG es más estable (Cheney y Doblar, 1982; Heburn y La Violette, 1990; Perkins et al., 1990; GarcíaLafuente et al., 1998; Baldacci et al., 2001; Vargas-Yáñez et al., 2002). Cuando uno o ambos giros están presentes, situación más frecuente en verano, el AJ discurre alrededor de los mismos y hacia el este. Cuando los giros desaparecen, usualmente en invierno, el AJ fluye paralelo a la 
costa africana septentrional, una situación denominada "modo costero" o "coastal mode" (Vargas-Yáñez et al., 2002).

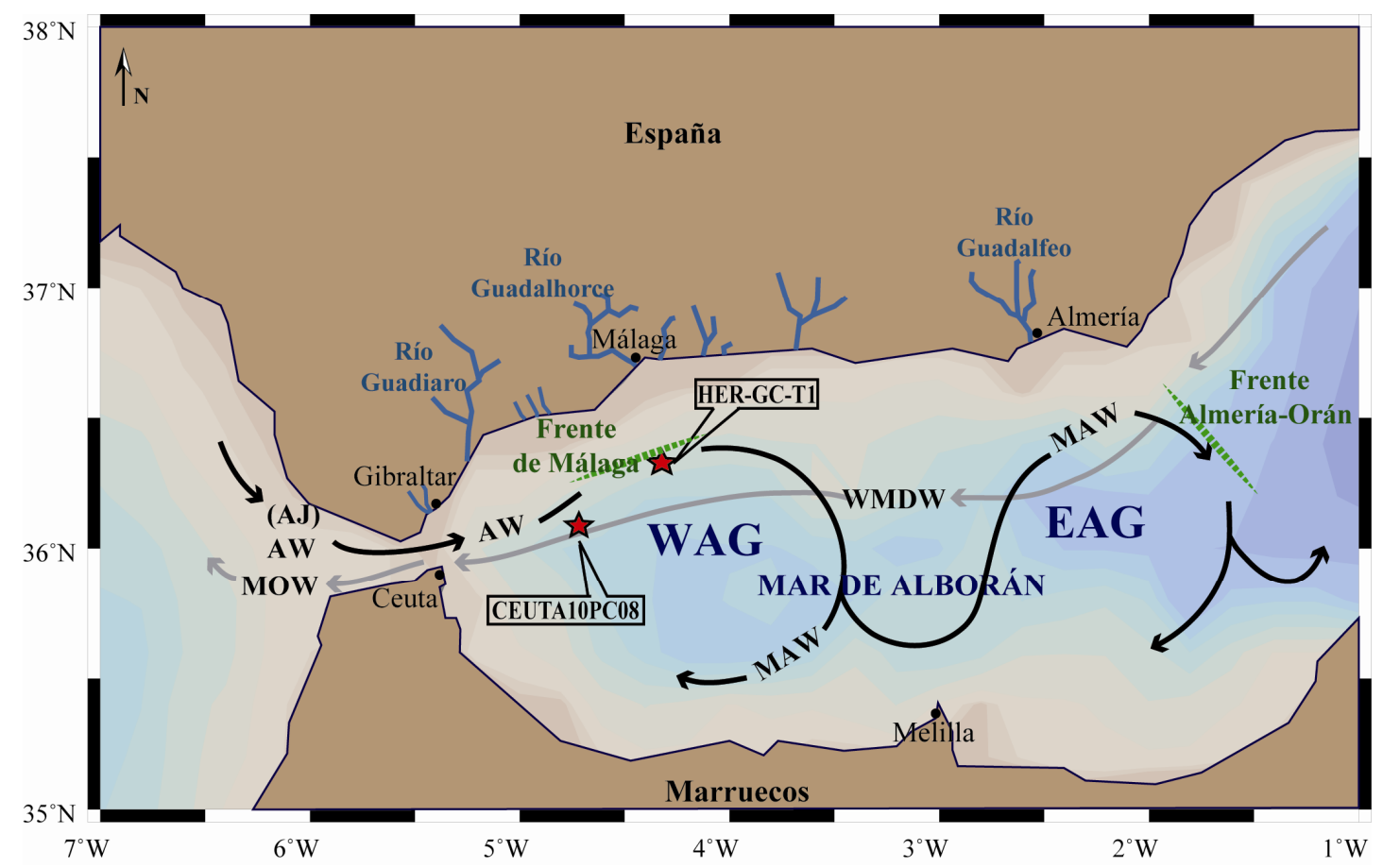

Figura 1.5. Mapa del mar de Alborán y sus principales características hidrográficas. Las estrellas rojas representan la localización de los testigos de sedimento utilizados en esta memoria: HER-GC-T1 y CEUTA10PC08. Leyenda: AW: Atlantic Water, AJ: Atlantic Jet, MAW: Modified Atlantic Water, WMDW: Western Mediterranean Deep Water, WAG: Western Anticyclonic Gyre, EAG: Eastern Anticyclonic Gyre.

La convergencia de la AW y la MAW en el límite septentrional de ambos giros genera dos frentes de densidad: el frente de Málaga asociado al WAG (Cheney, 1978) y el frente de Almearía-Orán asociado al EAG (Tintoré et al., 1988).

Los dos testigos sedimentarios utilizados en este trabajo (HER-GC-T1 y CEUTA10PC08) se localizan en el área de influencia del AJ y el WAG, por lo que este giro cobrará especial importancia en el desarrollo de esta tesis (Figura 1.5).

\subsection{2.-Productividad en el mar de Alborán}

La AW entrante es pobre en nutrientes, al igual que la MAW y al contrario que las aguas profundas que abandonan la cuenca (Macías et al., 2006). Los más de 200 metros que ocupa la MAW abarcan la mayor parte de la capa fótica, lugar en el que los productores primarios realizan la fotosíntesis. A consecuencia de esta escasez de nutrientes el mar Mediterráneo se caracteriza por una oligotrofía generalizada y baja productividad primaria. Sin embargo, este carácter oligotrófico no es extrapolable al mar de Alborán (Figura 1.6), donde la presencia de 
los giros WAG y EAG, sus frentes asociados y otros procesos físicos conllevan un aumento de la productividad biológica.

Estacionalmente, los máximos de concentración de nutrientes y de biomasa algal se dan en primavera, mientras que los mínimos se dan en verano (Bosc et al., 2004; Ramírez et al., 2005) (Figura 1.7).

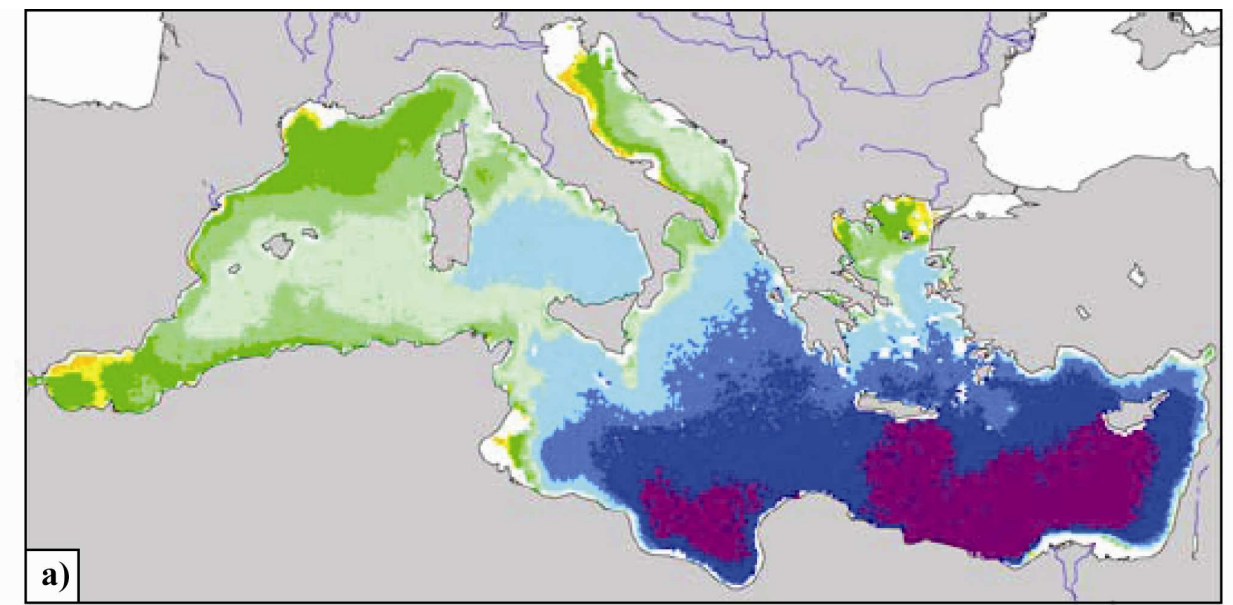

\section{Concentración media anual de clorofila- $a$ superficial $\left(\mathrm{mg}^{*} \mathrm{~m}^{-3}\right)$}
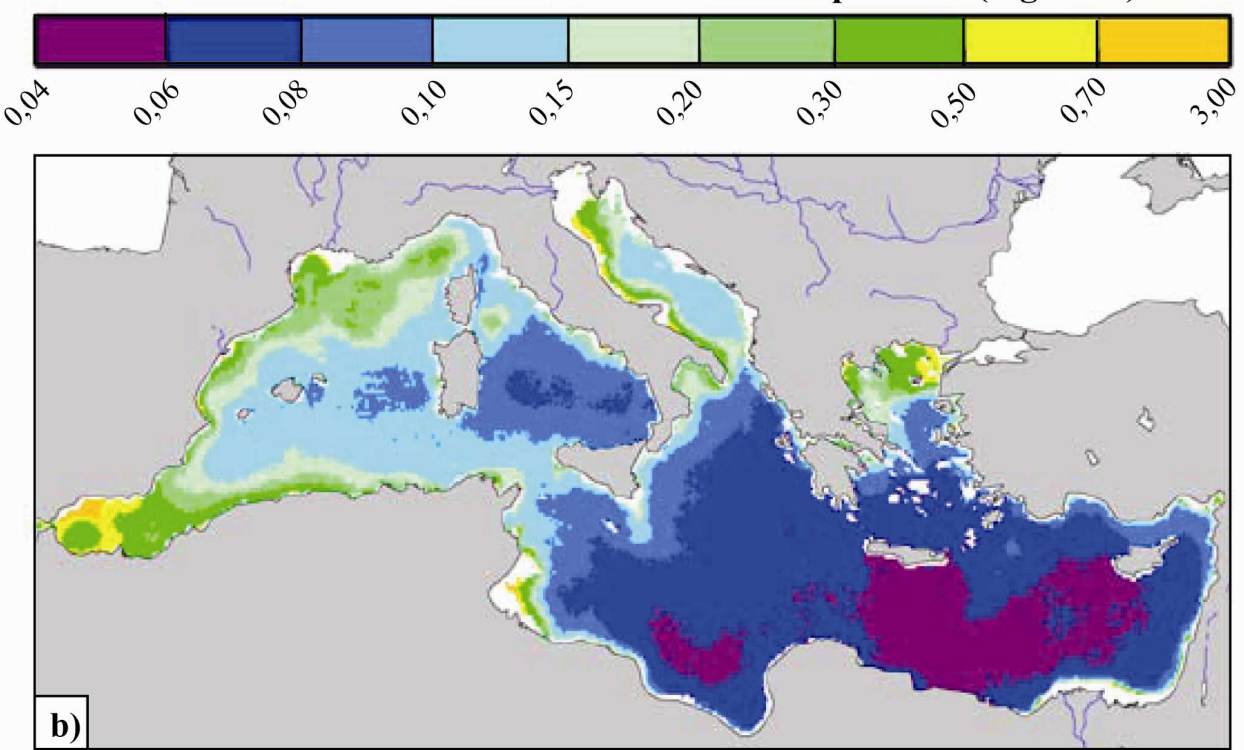

Producción primaria anual $\left(\mathrm{gC}^{*} \mathrm{~m}^{-2} * \mathrm{a}^{-1}\right)$

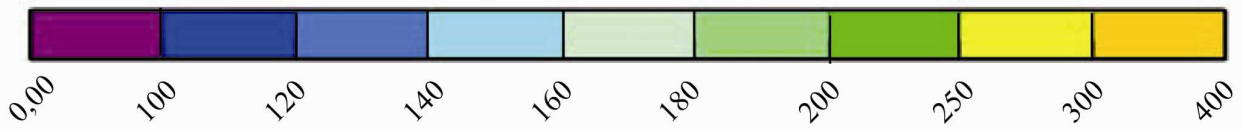

Figura 1.6. Mapas de la concentración media anual de: a) clorofila- $a$ superficial $\left(\mathrm{mg}^{*} \mathrm{~m}^{-3}\right)$ y b) producción primaria anual $\left(\mathrm{gC}^{*} \mathrm{~m}^{-2} * \mathrm{a}^{-1}\right)$ en el mar Mediterráneo. Modificado de Bosc et al. (2004). 

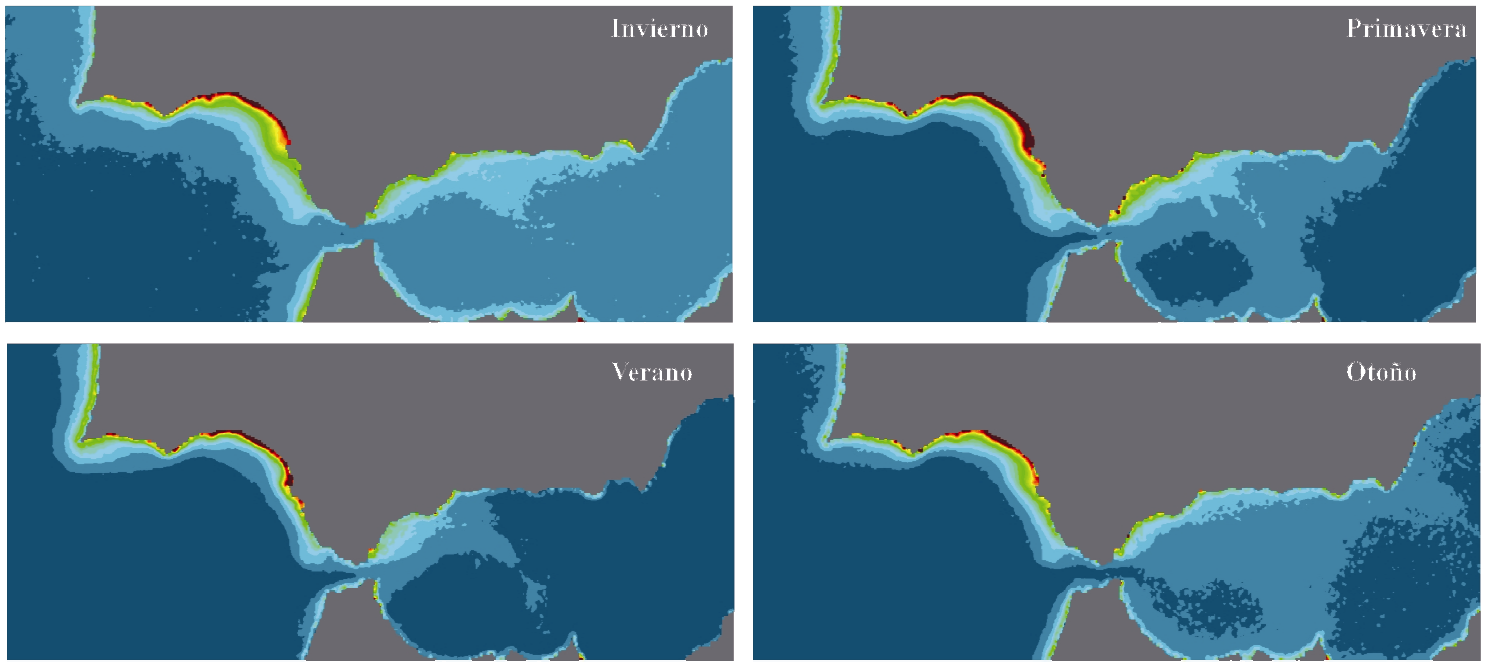

Concentración de clorofila- $a\left(\mathbf{m g}^{\star \star} \mathrm{cm}^{-3}\right)$

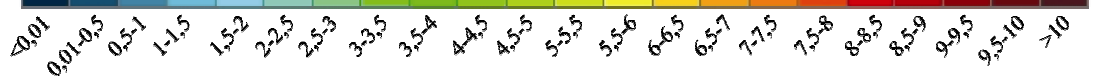

Figura 1.7. Medias estacionales de la concentración de clorofila- $a\left(\mathrm{mg}^{*} \mathrm{~cm}^{-3}\right)$ en el mar de Alborán entre los años 2000 y 2013 correspondientes al conjunto de imágenes de satélite AQUA MODIS. Imágenes extraídas de http://www.juntadeandalucia.es/medioambiente/.

En esta subcuenca el afloramiento (upwelling) (ascensión vertical por la columna de agua) de aguas profundas, frías y ricas en nutrientes, fertiliza la capa fótica (Macías et al., 2006) posibilitando la eclosión de productores primarios.

El afloramiento de aguas profundas y otros procesos responsables de inyectar nutrientes en la capa fótica pueden estar motivados por procesos físicos de diversa naturaleza. En el mar de Alborán, y para el estudio que nos ocupa, se destacan los siguientes:

i) La fertilización del AJ mediante intensa mezcla vertical en el estrecho de Gibraltar. La elevada velocidad horizontal del chorro es la responsable de distribuir por advección los nutrientes a otras zonas de Alborán y provocar la respuesta biológica (Ruiz et al., 2001; Navarro et al., 2011).

ii) El desplazamiento hacia el sur del AJ. Esto dejaría un "espacio" al norte del chorro que sería ocupado por el afloramiento de aguas más profundas (García-Gorriz y Carr, 1999; Sarhan et al., 2000; Navarro et al., 2011).

iii) Los vientos de poniente que soplan a lo largo de las costas meridionales españolas desplazan las aguas costeras mar adentro. El espacio que dejan es ocupado después por las aguas inmediatamente más profundas (García-Gorriz y Carr, 1999; Sarhan et al., 2000; Navarro et al., 2011). Este proceso se llama "afloramiento costero", dominante en la plataforma continental del noroeste del mar de Alborán. 


\subsection{3.-Importancia de los factores atmosféricos en el mar de Alborán}

Las variaciones de presión atmosférica a nivel del mar cobran especial relevancia (Cheney y Doblar, 1982; Candela y Lozano, 1991; García-Lafuente et al., 1998, entre otros) a la hora de explicar el estrecho acoplamiento entre los procesos físicos y biológicos en el mar de Alborán (Echevarría et al., 2002; Macías et al., 2006; Jordi et al., 2009; Vázquez et al., 2009) descritos en la sección anterior. Estas fluctuaciones dirigen el intercambio de aguas restringido por el estrecho de Gibraltar (García-Lafuente et al., 2002) y la intensidad de los vientos en el Mediterráneo occidental (Leaman y Schott, 1991).

Las conclusiones de los muchos trabajos que han investigado esta conexión (Macías et al., 2008) pueden resumirse de la siguiente manera: una reducción de la presión atmosférica a nivel del mar provoca una intensificación de los ponientes y aumento del influjo atlántico. Por el contrario, un aumento de la presión atmosférica provoca un debilitamiento de los ponientes y una reducción del flujo atlántico.

\section{4.-TELECONEXIÓN ATMOSFÉRICA CON EL ATLÁNTICO NORTE}

El término "clima mediterráneo" se incluye como referente en la clasificación de los tipos de climas de la Tierra (Köppen, 1900), utilizándose para describir el clima de aquellas regiones caracterizadas por inviernos suaves y húmedos y veranos cálidos y secos. Debido a la localización del Mediterráneo, este patrón general sufre variaciones espaciales y temporales dirigidas por las dinámicas climáticas tanto de latitudes medias, como la Oscilación del Atlántico Norte (North Atlantic Oscillation [NAO]) (Figura 1.8), así como de latitudes tropicales, como Oscilación del Sur de El Niño (El Niño-Southern Oscillation [ENSO]) y los monzones (Trigo et al., 2006).

Entre éstas, la NAO cobra especial relevancia en relación a la dinámica climática del Mediterráneo occidental. En términos generales, se trata de un patrón de variabilidad atmosférica que afecta a la región del Atlántico norte y los continentes adyacentes (Hurrell, 1995), especialmente en invierno (diciembre-marzo), produciendo grandes cambios atmosféricos y oceánicos (Hurrell y Deser, 2009). El índice NAO expresa este patrón bipolar midiendo la diferencia de presión a nivel del mar en invierno entre el centro de bajas presiones de Islandia y el centro de altas presiones de Azores (Figura 1.8). La fase negativa se corresponde con un debilitamiento de dicho sistema y una migración hacia el sur del cinturón de de ponientes, provocando inviernos suaves y húmedos en el sur de Europa. Por el contrario, la fase positiva de la NAO se corresponde con un refuerzo del sistema de presiones entre Islandia y Azores: el cinturón de vientos de componente oeste (ponientes) se desplaza hacia el norte y los inviernos en el sur de Europa son fríos y secos (Trigo et al., 2004; Hurrell y Deser, 2009). 

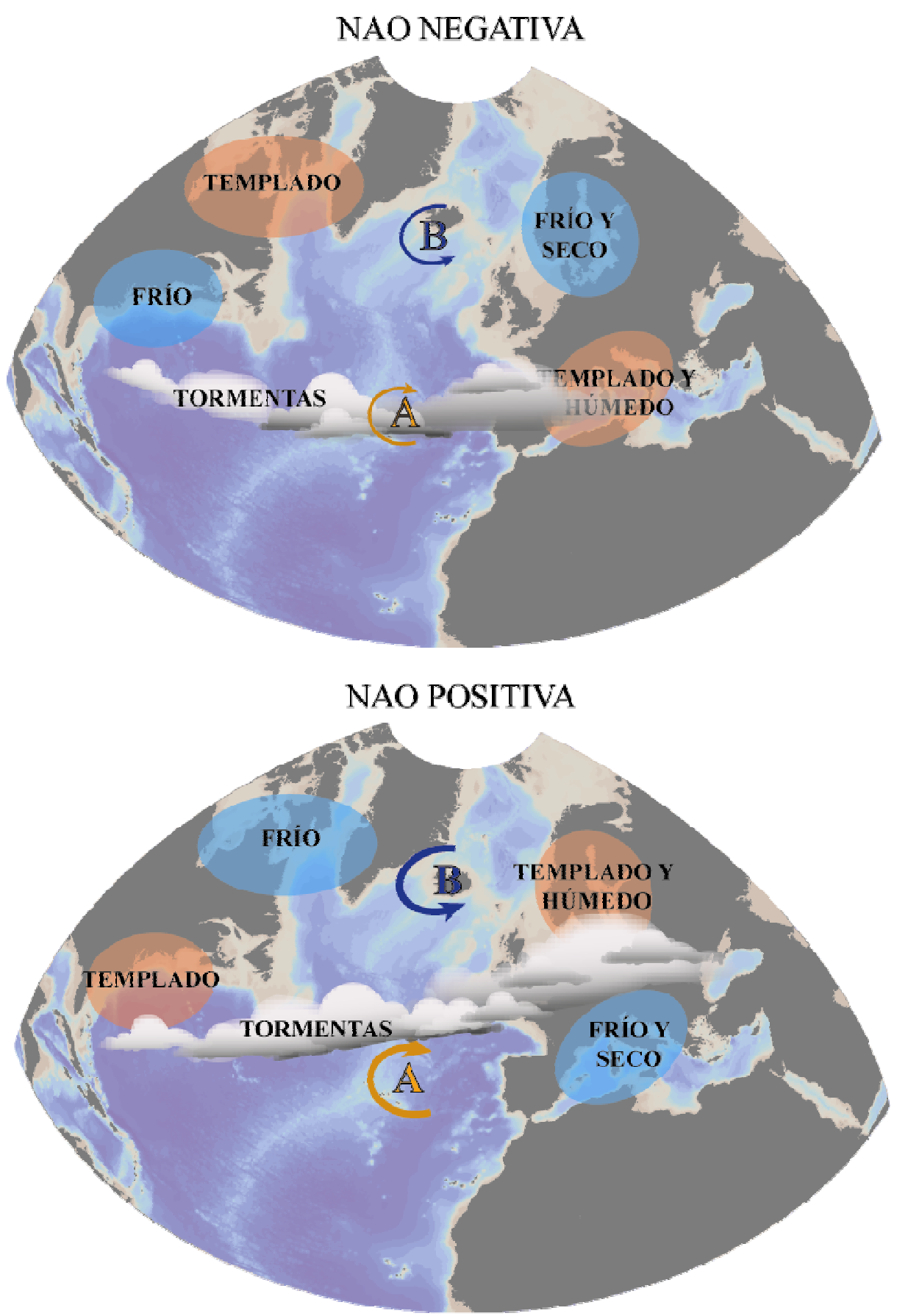

Figura 1.8. Esquemas de las fases negativa y positiva del índice de la Oscilación del Atlántico Norte (North Atlantic Oscillation [NAO]). La fase negativa (imagen superior) muestra los centros de presiones B y A (bajas y altas presiones respectivamente) debilitados. La fase positiva (imagen inferior) muestra los centros de presiones B y A reforzados.

En el contexto del Mediterráneo occidental, la influencia de la NAO ha sido observada en relación a las variaciones del nivel del mar (Tsimplis y Josey, 2001; Gomis et al., 2006), temperatura superficial del mar (sea surface temperature; SST) (Báez et al., 2013) precipitación, descarga fluvial y reservorios de agua (Rodó et al., 1997; Mariotti et al., 2002; Trigo et al., 2004; Vicente-Serrano et al., 2011) y el flujo neto de AW en el estrecho de Gibraltar (Fenoglio 2013). 


\section{Referencias}

Báez JC, Gimeno L, Gómez-Gesteira M, Ferri-Yáñez F y Real R. (2013) Combined Effects of the North Atlantic Oscillation and the Arctic Oscillation on Sea Surface Temperature in the Alborán Sea. PLoS ONE 8: e62201.

Baldacci A, Corsini G, Grasso R, Manzella G, Allen JT, Cipollini P, Guymer TH y Snaith HM. (2001) A study of the Alboran sea mesoscale system by means of empirical orthogonal function decomposition of satellite data. Journal of Marine Systems 29: 293-311.

Bèthoux JP. (1980) Mean water fluxes across sections in the Mediterranean Sea, evaluated on the basis of water and salt budgets and of observed salinities. Oceanologica Acta 3: 7988.

Bèthoux JP. (1979) Budgets of the Mediterranean Sea. Their dependance on the local climate and on the characteristics of the Atlantic waters Oceanologica Acta 2: 157-163.

Bosc E, Bricaud A y Antoine D. (2004) Seasonal and interannual variability in algal biomass and primary production in the Mediterranean Sea, as derived from 4 years of SeaWiFS observations. Global Biogeochemical Cycles 18: GB1005.

Bryden HL y Kinder TH. (1991) Recent progress in strait dynamics. Reviews of Geophysics 29: 617-631.

Cacho I, Grimalt JO y Canals M. (2002) Response of the Western Mediterranean Sea to rapid climatic variability during the last 50,000 years: a molecular biomarker approach. Journal of Marine Systems 33-34: 253-272.

Candela J y Lozano CJ. (1991) Barotropic Response of the Western Mediterranean to Observed Atmospheric Pressure Forcing. Seasonal and Interannual Variability of the Western Mediterranean Sea. American Geophysical Union 24: 325-359.

Cramp A y O'Sullivan G. (1999) Neogene sapropels in the Mediterranean: a review. Marine Geology 153: 11-28.

Cheney RE. (1978) Recent observations of the Alboran Sea frontal system. Journal of Geophysical Research: Oceans 83: 4593-4597.

Cheney RE y Doblar RA. (1982) Structure and variability of the alboran sea frontal system. Journal of Geophysical Research: Oceans 87: 585-594.

Echevarría F, García-Lafuente J, Bruno M, Gorsky G, Goutx M, González N, García CM, Gámez F, Vargas JM, Picheral M, Striby L, Varela M, Alonso JJ, Reul A, Cózar A, Prieto L, Sarhan T, Plaza F y Jiménez-Gámez F. (2002) Physical-biological coupling in the Strait of Gibraltar. Deep Sea Research Part II: Topical Studies in Oceanography 49: 4115-4130.

Estrada M y Margalef R. (1988) Supply of nutrients to the Mediterranean photic zone along a persistent front. Oceanologica Acta 9: 133-142. 
Fenoglio-Marc L, Mariotti A, Sannino G, Meyssignac B, Carillo A, Struglia MV y Rixen M. (2013) Decadal variability of net water flux at the Mediterranean Sea Gibraltar Strait. Global and Planetary Change 100: 1-10.

Font J, Palanques A, Puig P, Salat J y Emelianov M. (2007) Sequence of hydrographic changes in NW Mediterranean deep water due to the exceptional winter of 2005. Scientia Marina 71: 339-346.

Font J, Salat J y Tintoré J. (1988) Permanent features of the circulation in the Catalan Sea (Northwestern Mediterranean). Oceanologica Acta 9: 51-57.

García-Gorriz E y Carr ME. (1999) The climatological annual cycle of satellite-derived phytoplankton pigments in the Alboran Sea: a physical interpretation. Geophysical Research Letters 26: 2985-2988.

García-Lafuente J, Álvarez Fanjul E, Vargas JM y Ratsimandresy AW. (2002) Subinertial variability in the flow through the Strait of Gibraltar. Journal of Geophysical Research: Oceans 107 (C10): 3168.

García-Lafuente J, Cano N, Vargas M, Rubén JP y Hernández-Guerra A. (1998) Evolution of the Alboran Sea hydrographic structures during July 1993. Deep Sea Research Part I: Oceanographic Research Papers 45: 39-65.

Gomis D, Tsimplis MN, Martín-Míguez B, Ratsimandresy AW, García-Lafuente J y Josey SA. (2006) Mediterranean Sea level and barotropic flow through the Strait of Gibraltar for the period 1958-2001 and reconstructed since 1659. Journal of Geophysical Research: Oceans 111: C11005.

Heburn GW y La Violette PE. (1990) Variations in the Structure of the Anticyclonic Gyres Found in the Alboran Sea. Journal of Geophysical Research 95: 1599-1613.

Hurrell JW. (1995) Decadal Trends in the North Atlantic Oscillation: Regional Temperatures and Precipitation. Science 269: 676-679.

Hurrell JW y Deser C. (2009) North Atlantic climate variability: The role of the North Atlantic Oscillation. Journal of Marine Systems 78: 28-41.

Jordi A, Basterretxea G y Anglès S. (2009) Influence of ocean circulation on phytoplankton biomass distribution in the Balearic Sea: Study based on Sea-viewing Wide Field-ofview Sensor and altimetry satellite data. Journal of Geophysical Research: Oceans 114: C11005.

Köppen W. (1900) Versuch einer klassifikation der klimate, vorzugsweise nach ihren beziehungen zur pflanzenwelt. Geographische Zeitschrift 6: 657-679.

La Violette PE. (1990) The Western Mediterranean Circulation Experiment (WMCE): Introduction. Journal of Geophysical Research: Oceans 95: 1511-1514. 
Lascaratos A, Williams RG y Tragou E. (1993) A mixed-layer study of the formation of Levantine intermediate water. Journal of Geophysical Research: Oceans 98: 1473914749 .

Leaman KD y Schott FA. (1991) Hydrographic Structure of the Convection Regime in the Gulf of Lions: Winter 1987. Journal of Physical Oceanography 21: 575-598.

Lionello P, Malanotte-Rizzoli P, Boscolo R, Alpert P, Artale V, Li L, Luterbacher J, May W, Trigo R, Tsimplis M, Ulbrich U, Xoplaki E, P. Lionello PM-R y Boscolo R. (2006) The Mediterranean climate: An overview of the main characteristics and issues. Developments in Earth and Environmental Sciences. Elsevier, 1-26.

Locarnini R, Mishonov A, Antonov J, Boyer T, García H, Baranova O, Zweng M y Johnson D. (2010) World Ocean Atlas 2009, Volume 1, Temperature. En: S. Levitus (ed), 184 pp. US Government Printing Office, Washington, DC.

Locarnini, RA, AV. Mishonov, JI. Antonov, TP. Boyer, HE. García, OK. Baranova, MM. Zweng, CR. Paver, JR. Reagan, DR. Johnson, M. Hamilton, y D. Seidov (2013). World Ocean Atlas 2013, Volume 1: Temperature. En: S. Levitus y A. Mishonov (eds) NOAA Atlas NESDIS 73, 40 pp.

Macías D, Bruno M, Echevarría F, Vázquez A y García CM. (2008) Meteorologically-induced mesoscale variability of the North-western Alboran Sea (southern Spain) and related biological patterns. Estuarine, Coastal and Shelf Science 78: 250-266.

Macías D, García CM, Echevarría Navas F, Vázquez-López-Escobar A y Bruno Mejías M. (2006) Tidal induced variability of mixing processes on Camarinal Sill (Strait of Gibraltar): A pulsating event. Journal of Marine Systems 60: 177-192.

Mariotti A, Struglia MV, Zeng N y Lau KM. (2002) The Hydrological Cycle in the Mediterranean Region and Implications for the Water Budget of the Mediterranean Sea. Journal of Climate 15: 1674-1690.

MEDOCGROUP. (1970) Observation of formation of deep water in the Mediterranean Sea, 1969. Nature 227: 1037-1040.

Mertens C y Schott F. (1998) Interannual Variability of Deep-Water Formation in the Northwestern Mediterranean. Journal of Physical Oceanography 28: 1410-1424.

Millot C. (1985) Some features of the Algerian Current. Journal of Geophysical Research: Oceans 90: 7169-7176.

Millot C. (1992) Are there major differences between the largest mediterranean seas? A preliminary investigation. Bulletin de l'Institut Océanographique, Monaco 11: 3-25.

Millot C. (1999) Circulation in the Western Mediterranean Sea. Journal of Marine Systems 20: 423-442. 
Navarro G, Vázquez Á, Macías D, Bruno M y Ruiz J. (2011) Understanding the patterns of biological response to physical forcing in the Alborán Sea (western Mediterranean). Geophysical Research Letters 38.

Parrilla G, Kinder TH y Preller RH. (1986) Deep and intermediate mediterranean water in the western Alboran Sea. Deep Sea Research Part A. Oceanographic Research Papers 33: $55-88$.

Perkins H, Kinder T y Violette PL. (1990) The Atlantic inflow in the Western Alboran Sea. Journal of Physical Oceanography 20: 242-263.

Ramírez T, Cortés D, Mercado JM, Vargas-Yáñez M, Sebastián M y Liger E. (2005) Seasonal dynamics of inorganic nutrients and phytoplankton biomass in the NW Alboran Sea. Estuarine, Coastal and Shelf Science 65: 654-670.

Rixen M, Beckers JM, Levitus S, Antonov J, Boyer T, Maillard C, Fichaut M, Balopoulos E, Iona S, Dooley H, García MJ, Manca B, Giorgetti A, Manzella G, Mikhailov N, Pinardi N y Zavatarelli M. (2005) The Western Mediterranean Deep Water: A proxy for climate change. Geophysical Research Letters 32: L12608.

Rodó X, Baert E y Comín FA. (1997) Variations in seasonal rainfall in Southern Europe during the present century: relationships with the North Atlantic Oscillation and the El NiñoSouthern Oscillation. Climate Dynamics 13: 275-284.

Ruiz J, Echevarría F, Font J, Ruiz S, García E, Blanco JM, Jiménez-Gómez F, Prieto L, González-Alaminos A, García CM, Cipollini P, Snaith H, Bartual A, Reul A y Rodríguez V. (2001) Surface distribution of chlorophyll, particles and gelbstoff in the Atlantic jet of the Alborán Sea: from submesoscale to subinertial scales of variability. Journal of Marine Systems 29: 277-292.

Sarhan T, García-Lafuente J, Vargas M, Vargas JM y Plaza F. (2000) Upwelling mechanisms in the northwestern Alboran Sea. Journal of Marine Systems 23: 317-331.

Schlitzer R. (2014) Ocean Data View, http://odv.awi.de.

Smith RO, Bryden HL y Stansfield K. (2008) Observations of new western Mediterranean deep water formation using Argo floats 2004-2006. Ocean Science 2: 133-149.

The Donde Va Group. (1984) Donde va? An oceanographic experiment in the Alboran Sea. Eos, Transactions American Geophysical Union 65: 682-683.

Thunell RC y Williams DF. (1989) Glacial-Holocene salinity changes in the Mediterranean Sea: hydrographic and depositional effects. Nature 338: 493-496.

Tintoré J, La Violette PE, Blade I y Cruzado A. (1988) A study of an intense density front in the eastern Alboran Sea: the Almeria-Oran front. Journal of Physical Oceanography 18: 1384-1397.

Trigo R, Xoplaki E, Zorita E, Luterbacher J, Krichak SO, Alpert P, Jacobeit J, Sáenz J, Fernández J, González-Rouco F, García-Herrera R, Rodo X, Brunetti M, Nanni T, 
Maugeri M, Türke§ M, Gimeno L, Ribera P, Brunet M, Trigo IF, Crepon M, Mariotti A, P. Lionello PM-R y Boscolo R. (2006) Chapter 3 Relations between variability in the Mediterranean region and mid-latitude variability. Developments in Earth and Environmental Sciences. Elsevier, 179-226.

Trigo RM, Pozo-Vázquez D, Osborn TJ, Castro-Díez Y, Gámiz-Fortis S y Esteban-Parra MJ. (2004) North Atlantic oscillation influence on precipitation, river flow and water resources in the Iberian Peninsula. International Journal of Climatology 24: 925-944.

Tsimplis MN y Josey SA. (2001) Forcing of the Mediterranean Sea by atmospheric oscillations over the North Atlantic. Geophysical Research Letters 28: 803-806.

Vargas-Yáñez M, Plaza F, García-Lafuente J, Sarhan T, Vargas JM y Vélez-Belchi P. (2002) About the seasonal variability of the Alboran Sea circulation. Journal of Marine Systems 35: 229-248.

Vázquez A, Flecha S, Bruno M, Macías D y Navarro G. (2009) Internal waves and short-scale distribution patterns of chlorophyll in the Strait of Gibraltar and Alborán Sea. Geophysical Research Letters 36: L23601.

Vicente-Serrano SM, Trigo RM, López-Moreno JI, Liberato MLR, Lorenzo-Lacruz J, Beguería S, Morán-Tejeda E y El Kenawy A. (2011) Extreme winter precipitation in the Iberian Peninsula in 2010: anomalies, driving mechanisms and future projections. Climate Research 46: 51-65.

Zweng MM, Reagan JR, Antonov JI, Locarnini RA, Mishonov AV, Boyer TP, García HE, Baranova OK, Johnson DR, Seidov D y Biddle MM. (2013) World Ocean Atlas 2013, Volume 2: Salinity. En: Levitus S y Mishonov A (eds) NOAA Atlas NESDIS 74, 39 pp. 
CAPÍTULO 2 



\section{2.-COCOLITÓFOROS}

\section{1.-INTRODUCCIÓN}

Los cocolitóforos son un grupo de algas pardas unicelulares perteneciente al filo Haptophyta y la clase Prymnesiophyceae (Hibberd, 1976). Incluyen todas las algas haptophytas que en algún momento de su ciclo de vida producen escamas calcificadas con las que cubren su célula llamadas cocolitos.

Estas algas habitan la parte superior de la columna de agua y a pesar de tener un tamaño inferior a las $30 \mu \mathrm{m}$ son uno de los componentes mayoritarios del fitoplancton marino llegando a alcanzar el $10 \%$ de su biomasa total (Tyrrell et al., 2009). Algunas especies como Emiliania huxleyi pueden proliferar y alcanzar los 10 millones de especímenes por litro. Una eclosión de estas algas microscópicas puede abarcar vastas extensiones de océano y teñir de verde lechoso su superficie haciéndose visibles desde el espacio (Figura 2.1).

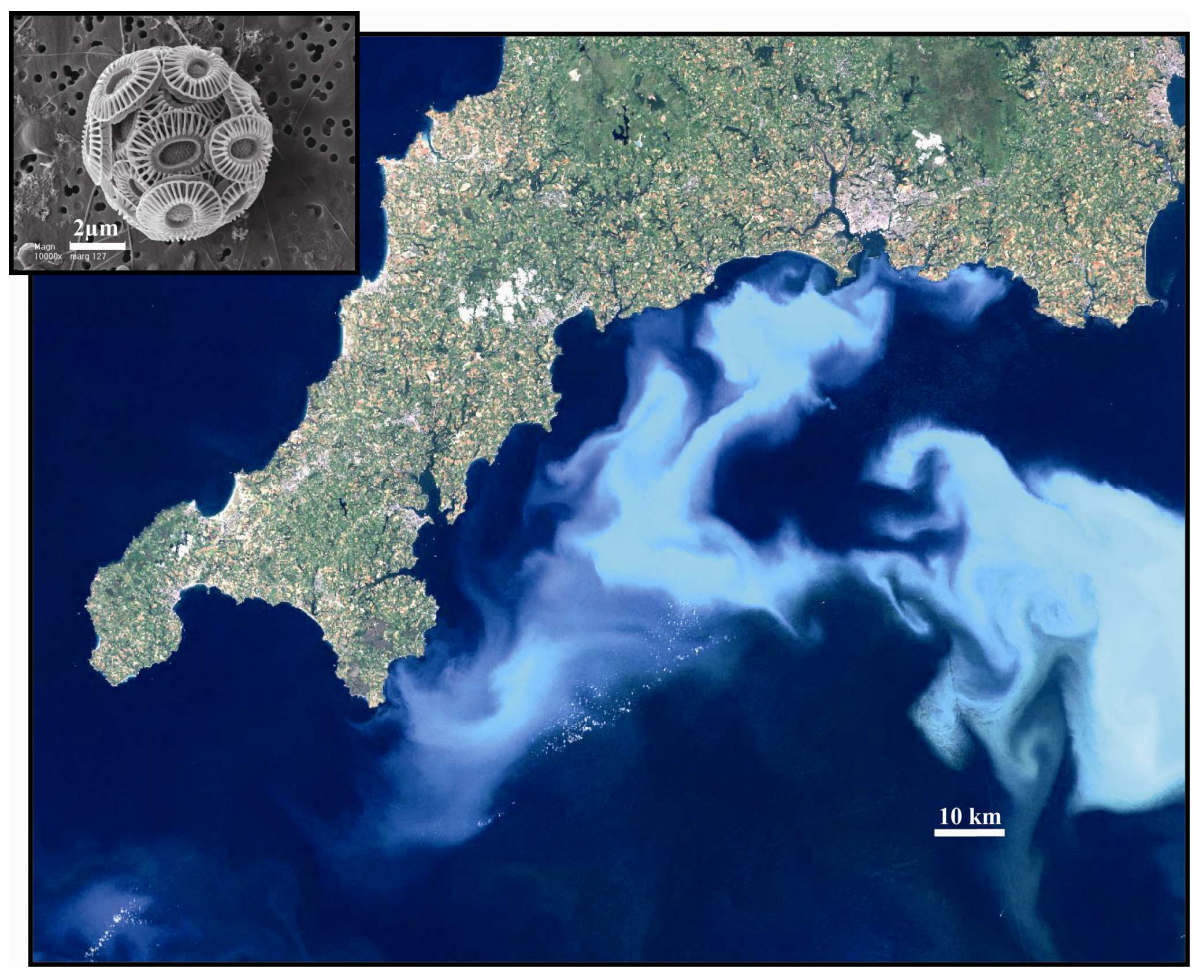

Figura 2.1. Imagen satelital de una eclosión o bloom de E. huxleyi, en las costas de Cornwall, Inglaterra, tomada el 24 de julio de 1999 por el satélite LANDSAT. Fuente: http://ina.tmsoc.org/galleries/colourcoccos/source/z00-1_bloom_summer_99.htm. Imagen de una cocosfera de E. huxleyi (arriba a la izquierda) al microscopio electrónico. Fuente: http://www.southampton.ac.uk/oes/.

El interés científico en estos diminutos organismos reside en el papel que desempeñan dentro de varios ciclos biogeoquímicos. Los cocolitóforos fijan grandes cantidades de $\mathrm{CO}_{2}$ atmosférico transformándolo en materia orgánica mediante la fotosíntesis. Son productores de dimetilsulfuro 
(DMS), un importante formador de núcleos de nubes (Keller, 1989; Malin y Erst, 1997). También son productores de diversos compuestos orgánicos como las alquenonas, moléculas de carbono de cadena larga cuyos ratios dependen de la temperatura superficial marina (Müller et al., 1998). A su vez, contribuyen al secuestro y almacenamiento de $\mathrm{CO}_{2}$ atmosférico en los sedimentos marinos mediante la formación de cocolitos y su transporte a los fondos oceánicos (Honjo, 1976).

De preservarse, los cocolitos pasan a formar parte del archivo sedimentario convirtiéndose en indicadores estratigráficos clave (Lloyd et al., 2011) y almacenando con ellos una valiosa información para la reconstrucción paleoclimática y paleoceanográfica del ambiente en el que vivieron.

El término "nanofósiles calcáreos" engloba a todas las formas extintas del nanoplancton calcáreo. A excepción de los individuos retrabajados, la mayoría de los especímenes estudiados en esta tesis doctoral cuentan con representantes vivos en los océanos actuales, por lo que el término empleado para referirse a los mismos será "cocolitóforos". El término "especímenes retrabajados" (reworked specimens) se empleará para designar aquellos individuos pertenecientes a niveles estratigráficos más antiguos que los estudiados. Estos individuos fueron removilizados y posteriormente transportados por diferentes mecanismos desde su lugar de sedimentación original hasta el área de estudio, donde sedimentaron de nuevo.

\section{2.-ESTRUCTURA CELULAR}

La mayoría de los cocolitóforos son organismos unicelulares móviles o estáticos de forma cocoide. Algunas de las estructuras internas celulares más importantes contenidas en el protoplasma son: el núcleo, los cloroplastos, las mitocondrias y el aparato de Golgi (Figura 2.2). Normalmente los cloroplastos son uno o dos y contienen clorofila $a+c$ como pigmentos principales, además de carotenoides como la diadinoxantina o la fucoxantina (Brand, 1994).

Recubriendo el protoplasma se encuentra el periplasto, una pared celular formada por varias capas de pequeñas escamas que pueden tener origen orgánico o inorgánico (Figura 2.2) y de cuya síntesis se encarga el aparato de Golgi (Kiermayer y Romanovicz, 1981; Billard y Inouye, 2004).

Las escamas de origen totalmente orgánico son pequeñas y finas y aparecen inmediatamente después del protoplasma. Las escamas de origen inorgánico son más grandes y robustas y son visibles al microscopio óptico. Estas escamas son los cocolitos, los cuales se imbrican entre sí y se disponen normalmente conformando una única capa externa a las capas orgánicas que recibe el nombre de cocosfera.

Algunas especies de cocolitóforos móviles presentan un par de flagelos sin apéndices filamentosos (Figura 2.2) en la parte apical (Green y Hori, 1994). El aparato flagelar, lejos de 
ser una estructura simple, es considerado como una característica evolutiva y por tanto de importancia filogenética (Pienaar, 1994).

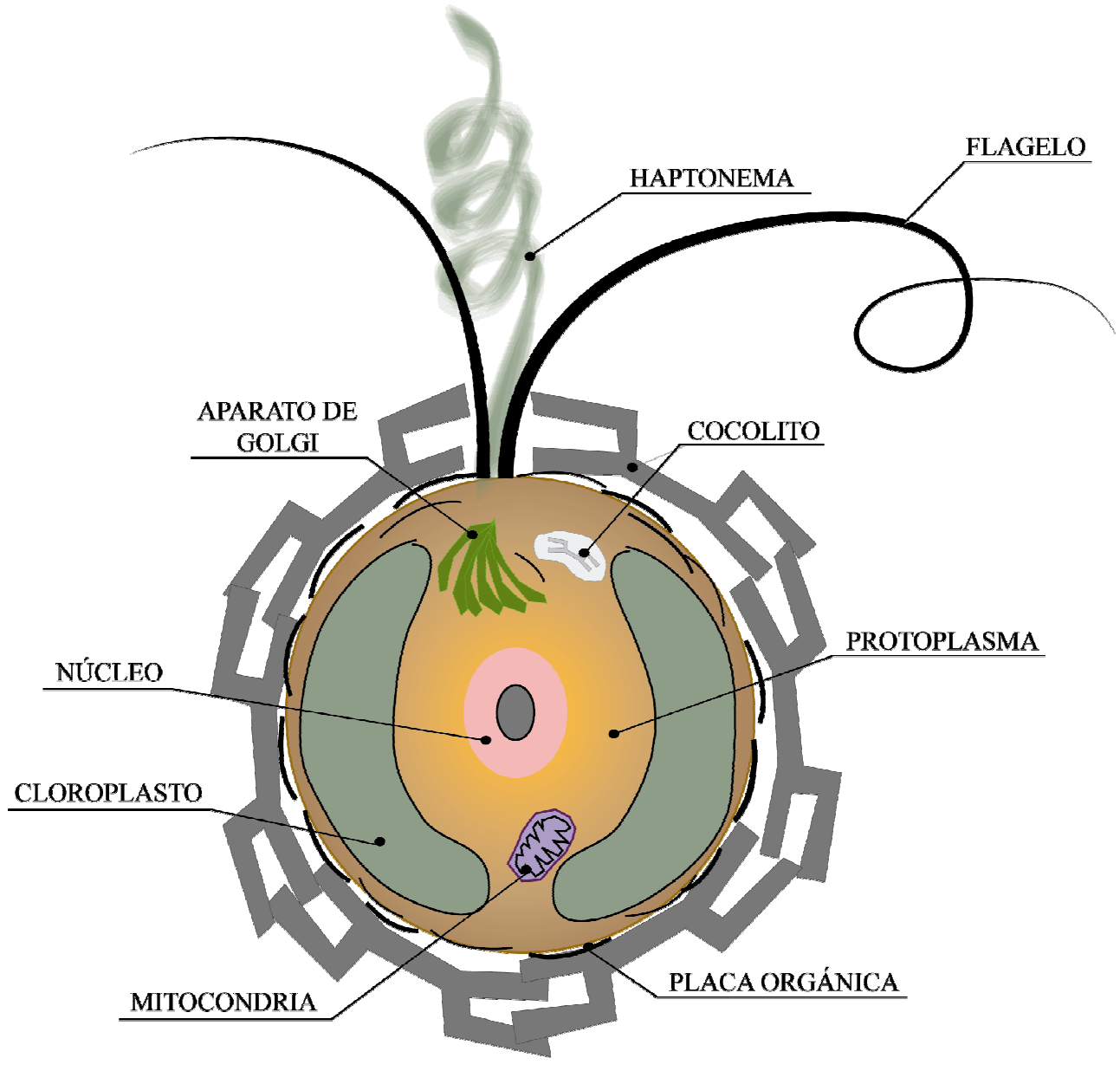

Figura 2.2. Representación esquemática de la estructura celular general de los cocolitóforos. Modificada de Young y Henriksen (2003).

Otra particularidad de los cocolitóforos es la presencia del haptonema en aquellas células con un estadio móvil (Figura 2.2). Los primeros estudios se refieren a él como un tercer flagelo central, hasta que Manton (1964) la reconoció como otra estructura. Se trata de un órgano multifuncional, de tamaño variable en función de la especie y capaz de doblarse, rotar o enrollarse. Está compuesto por un número variable de microtubos que aumenta hacia la base y está rodeado parcial o completamente por retículo endoplasmático. Junto a los flagelos forma el aparato flagelar, si bien es cierto que algunos cocolitóforos presentan un haptonema muy reducido o vestigial.

\subsection{1.-Cocolitos y sus tipologías}

Generalmente la cocolitogénesis es un proceso secuencial que comienza con el crecimiento del cocolito dentro o en las proximidades del aparato de Golgi (Pienaar, 1994) (Figura 2.2). 
Cuando el cocolito ha completado su calcificación es expulsado a la superficie de la célula (Figura 2.3) cerca del polo flagelar (Paasche, 2001).

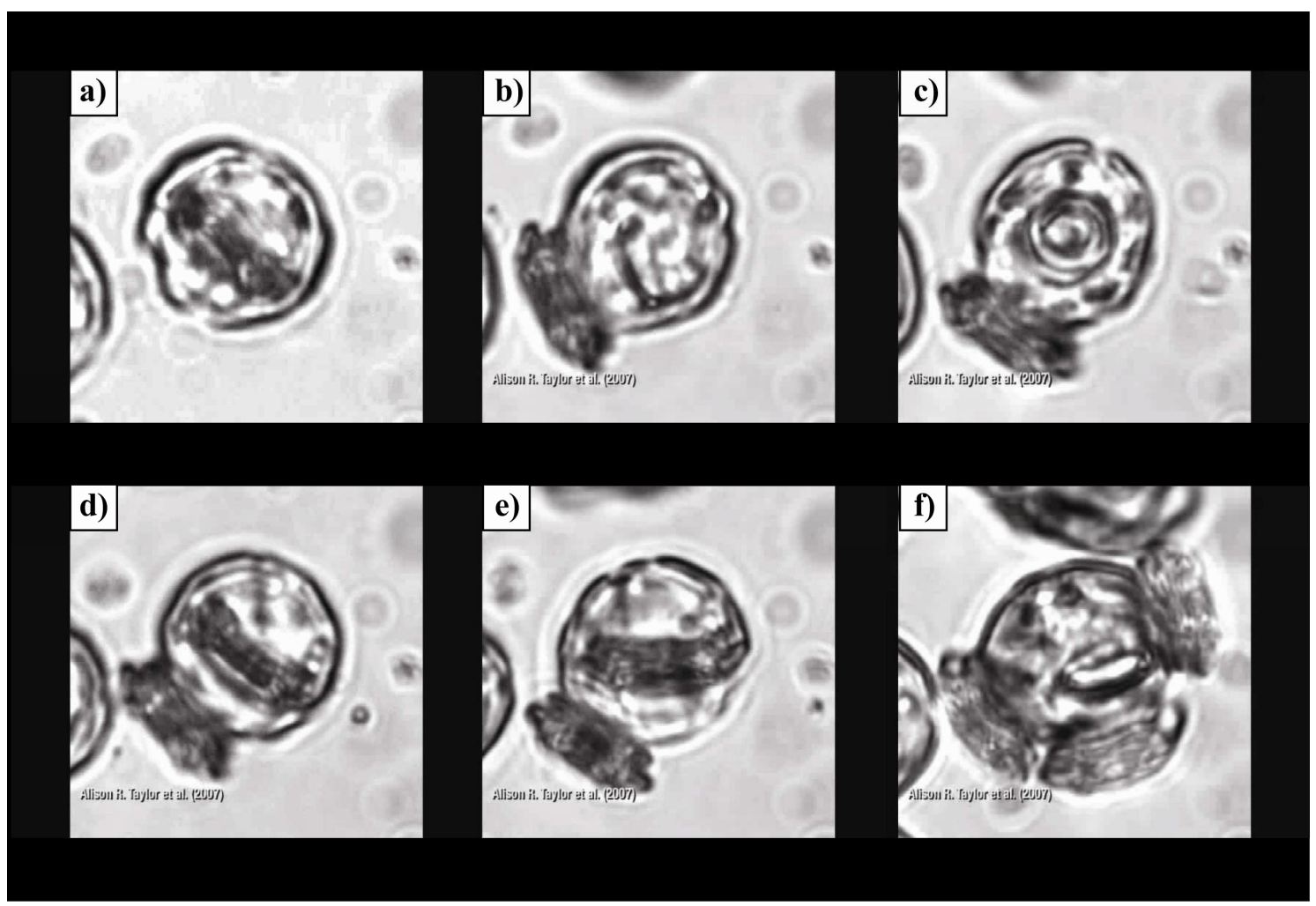

Figura 2.3. Cocolitogénesis en Coccolithus pelagicus subsp. braarudii. Imágenes tomadas del vídeo producido por Dr. Alison Taylor de Marine Biological Association, Plymouth. Fuente: http://ina.tmsoc.org/nannos/cpelmovie/index.html. a) Formación intracelular de un cocolito de $C$. pelagicus. b) Expulsión del cocolito recién formado al exterior de la célula. c), d y e) Formación intracelular del siguiente cocolito. f) Expulsión sucesiva de los cocolitos para la formación de la cocosfera.

La función de los cocolitos es desconocida. Algunas de las hipótesis incluyen la protección de la célula ante predadores, virus y bacterias, el control de la absorción de luz o de la flotabilidad de la célula y la regulación de procesos bioquímicos (Young, 1994; Flores y Sierro, 2007).

La morfología, estructura y ordenación de los cocolitos son exclusivas de cada especie por lo que dichas características se utilizan como principal criterio de clasificación. La orientación de las unidades cristalinas de cada cocolito bajo la luz polarizada del microscopio óptico produce una figura de interferencia que permite diferenciar los cocolitos a nivel de especie (Winter y Siesser, 1994; Thierstein et al., 2004).

A continuación se describen los 3 tipos existentes de cocolitos:

i) Heterococolitos: cocolitos formados por unidades cristalinas de calcita de formas y tamaños variables (Figura 2.4a). Dichos cristales se disponen radial y verticalmente formando 
un cocolito ovalado que consta de un borde y un área central, la cual puede estar vacía o presentar estructuras complejas (Young y Henriksen, 2003; Flores y Sierro, 2007). Se distinguen tres tipos de heterococolitos en función de las unidades cristalinas que los forman (Figura 2.4b):

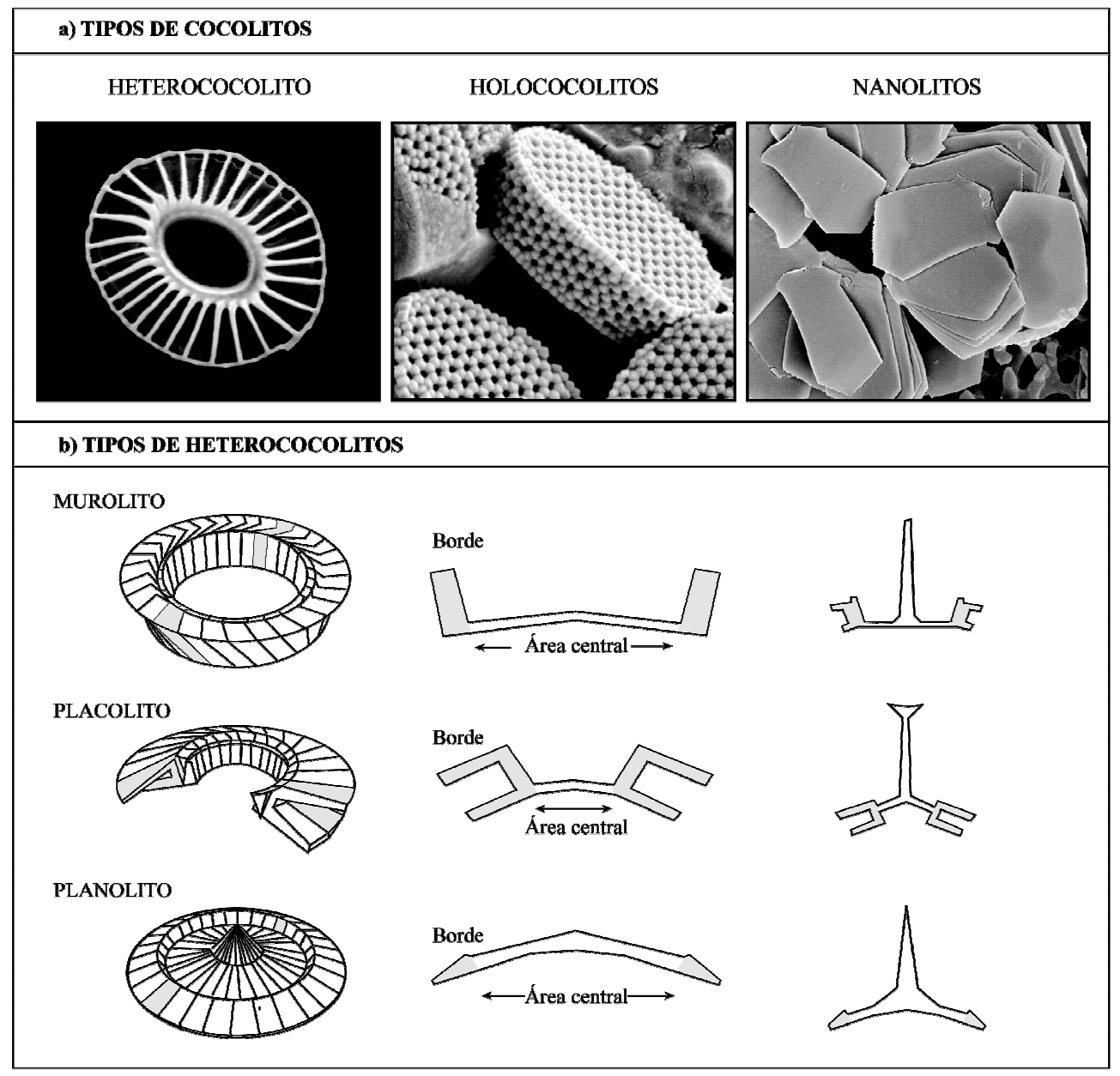

Figura 2.4. Tipología de los cocolitos. a) Tipos de cocolitos. b) Tipos de heterococolitos. Fuente: http://ina.tmsoc.org/Nannotax3/index.php?dir=Coccolithophores. http://ina.tmsoc.org/terminology/3coccoliths.htm. Modificada de

- Murolitos: tienen forma de cuenco y presentan un área central dilatada rodeada de una pared perpendicular variable.

- Placolitos: tienen forma de disco y su borde se compone de dos escudos (distal y proximal) separados por un tubo.

- Planolito: tienen forma de disco pero se componen de un único escudo de manera que el borde y el área central quedan en el mismo plano. 
ii) Holococolitos: cocolitos formados por numerosos cristales de calcita, pequeños e idénticos unos a otros que forman una placa ovalada (Young y Henriksen, 2003; Flores y Sierro, 2007) (Figura 2.4a).

iii) Nanolitos: este grupo incluye todas las estructuras calcáreas que difieren de las anteriores (Young y Henriksen, 2003; Flores y Sierro, 2007). Como ejemplos actuales, se encuentran los cocolitos de los génereos Florisphaera (placas) (Figura 2.4a) y Braarudosphaera (pentalitos).

\section{3.-CICLO DE VIDA}

Como ocurría con las características morfológicas de los cocolitóforos, la complejidad de sus ciclos de vida y sus fases son muy variables entre especies. De manera general su ciclo de vida es heteromórfico, alternando dos fases: una fase diploide con células que presentan dos copias de cada cromosoma $(2 n)$ y una fase haploide con células que contienen una sola copia (n) (Figura 2.5).

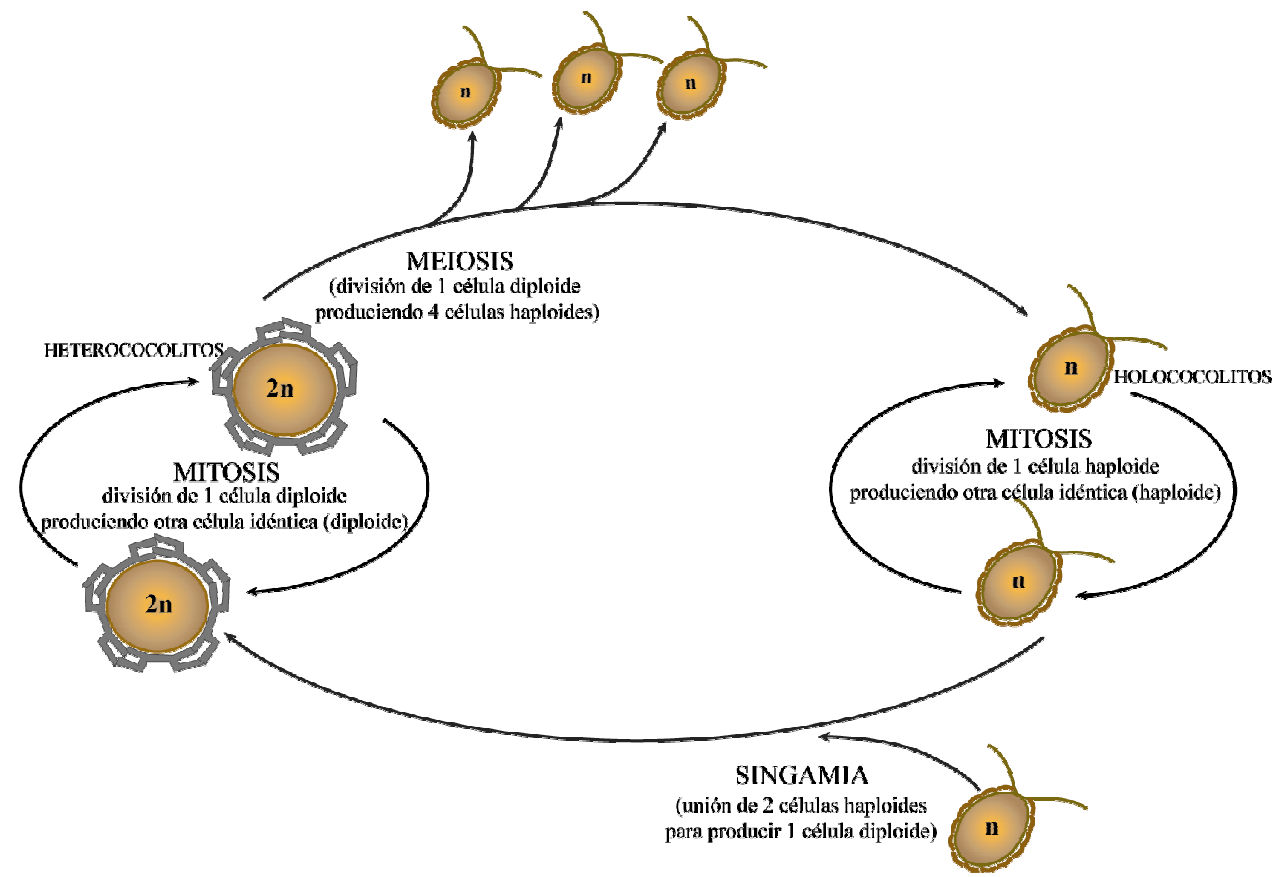

Figura 2.5. Representación esquemática del ciclo de vida de un cocolitóforo. $2 \mathrm{n}=$ célula diploide, $\mathrm{n}=$ célula haploide. Modificado de Flores y Sierro (2007).

La transición de la fase diploide a la haploide se produce por meiosis, dando lugar a cuatro células hijas haploides con una copia idéntica de cromosoma. La transición de la fase haploide a la diploide se produce por singamia, donde dos células haploides se combinan dando lugar a una célula diploide (Flores y Sierro, 2007). Ninguna de estas fases es necesariamente dominante, y en ambas puede darse reproducción asexual por mitosis, aparentemente de forma indefinida, hasta que algún factor ambiental desencadena la transición a la fase alternante del ciclo de vida (Young y Henriksen, 2003). 
Las células diploides pueden ser móviles o estáticas y presentar heterococolitos, mientras que las células haploides suelen ser móviles y pueden presentar holococolitos, nanolitos o escamas orgánicas.

\section{4.-ECOLOGÍA Y DISTRIBUCIÓN}

\subsection{1.-Factores limitantes}

Los cocolitóforos son organismos autótrofos y por lo tanto habitan únicamente en la capa fótica pues dependen de la existencia de luz para realizar la fotosíntesis (Zondervan, 2007). Su distribución también está altamente relacionada con la disponibilidad de nutrientes como el fosfato y el nitrato (Zondervan, 2007), si bien numerosas especies pueden proliferar en aguas oligotróficas.

Otros factores ambientales como la salinidad y la temperatura de las masas de agua afectan a su desarrollo, de forma que cada especie puede mostrar un rango de tolerancia y un óptimo específicos.

\subsection{2.-Distribución espacial}

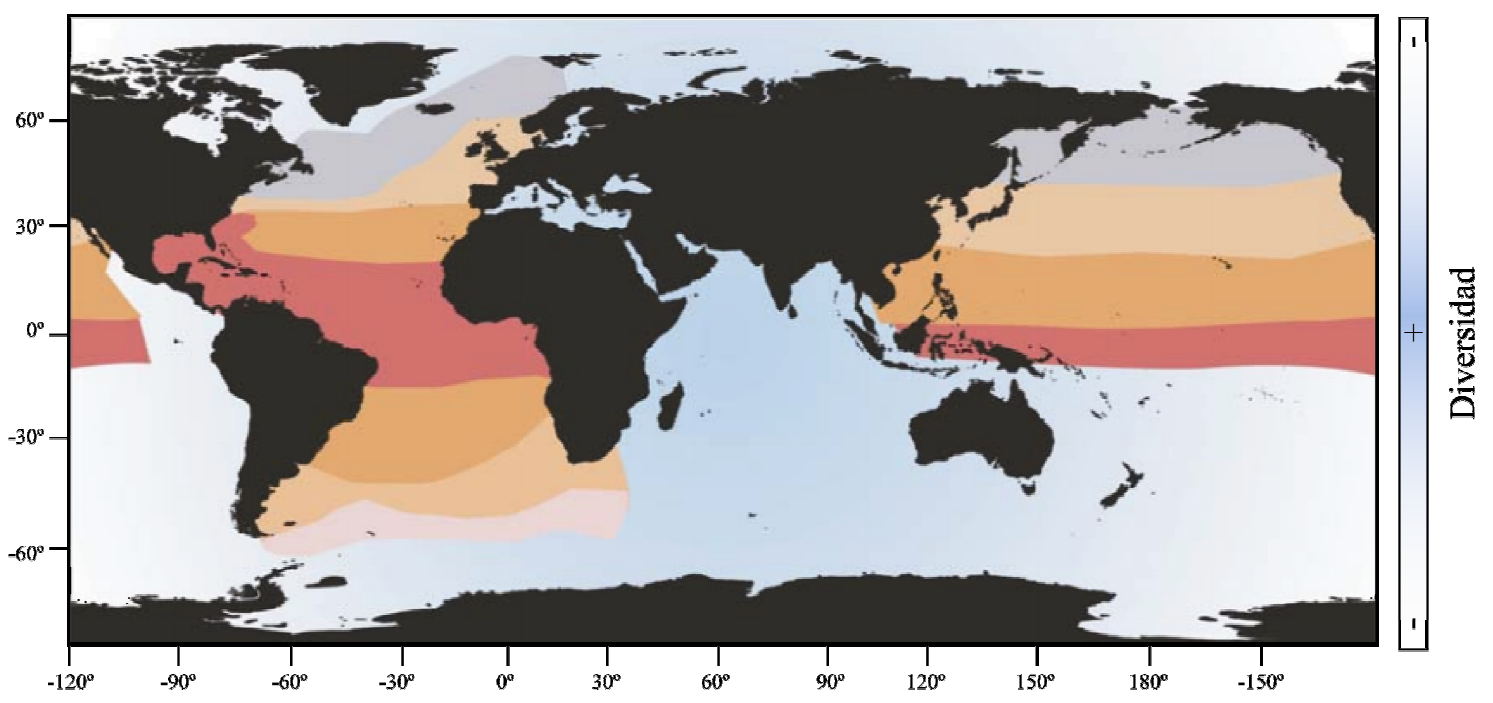

ZONAS BIOGEOGRÁFICAS DE LOS COCOLITÓFOROS:

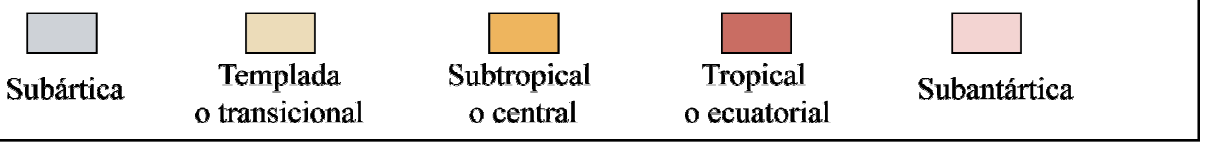

Figura 2.6. Zonas biogeográficas de la distribución espacial general de los cocolitóforos (McIntyre y Bé, 1967; Okada y Honjo, 1973). Modificada de Flores y Sierro (2007).

De manera muy general, la distribución espacial que siguen los cocolitos atiende a la diferenciación de cinco zonas biogeográficas principales definidas por su latitud: subártica, templada o transicional, subtropical o central, tropical o ecuatorial y subantártica (Figura 2.6). 
Dichas zonas fueron establecidas por McIntyre y Bé (1967) en el océano Atlántico y por Okada y Honjo (1973) en el océano Pacífico.

Tabla 2.1. Asociaciones cocolitoforales características de cada zona biogeográfica. Modificada de Jordan y Chamberlain (1997). Los taxones considerados en esta tesis doctoral están marcados con un asterisco $(*)$.

\section{Subártica}

Algirosphaera robusta, Balaniger balticus, Calciarcus alaskensis, Calciopappus caudatus, Coccolithus pelagicus*, Emiliania huxleyi*, Papposphaeraceae, Quaternariella obscura, Syracosphaera boreales, Wigwamma spp.

\section{Templada o transicional}

Balaniger balticus, Calcidiscus leptoporus*, Coccolithus pelagicus*, Emiliania huxleyi*, Ericiolus spp., Gephyrocapsa spp.*, Helicosphaera spp.*, Reticulofenestra spp., Syracosphaera spp*.

\section{Subtropical o central}

Alisphaera spp., Alveosphaera bimurata, Canistrolithus valliformis, Emiliania huxleyi*, Florisphaera profunda*, Gephyrocapsa oceanica*, Gladiolithus spp., Hayaster perplexus, Helicosphaera spp.*, Navilithus altivelum, Oolithotus fragilis*, Pontosphaeraceae*, Reticulofenestra sessilis, Rhabdosphaeraceae*, Solisphaera spp., Syracosphaeraceae*, Tetralithoides quadrilaminata, Turrilithus latericioides, Umbellosphaera spp.*, Umbilicosphaera spp.*, Vexillarius cancellifer

\section{Tropical o ecuatorial}

Alisphaera spp., Calcidiscus leptoporus*, Emiliania huxleyi*, Gephyrocapsa spp.*, Florisphaera profunda*, Gladiolithus spp., Reticulofenestra sessilis

\section{Subantártica}

Calciarcus alaskensis, Ericolus spp., Papposphaeraceae, Quatermariella obscura, Wigwamma spp.

En la tabla 2.1 se observan asociaciones cocolitoforales típicas de cada una de las zonas biogeográficas mencionadas. De la misma se desprende que la distribución de muchas especies no está restringida a una única zona biogeográfica, como es el caso de E. huxleyi, la cual puede encontrarse en asociaciones subárticas, templadas, subtropicales o tropicales. 


\subsection{3.-Distribución vertical}

Los nutrientes, la luz y la temperatura son algunos de los factores principales que determinan la distribución vertical de las especies cocolitoforales. La variación de estos factores en la columna de agua determina la profundidad de la nutriclina, la capa fótica y la termoclina respectivamente (Tabla 2.2). La mayoría de las especies habitan en la zona fótica superior (ZFS; 0-80 m). Sin embargo otras lo hacen a mayores profundidades, como Oolithotus fragilis que habita en la zona fótica media (ZFM; 80-120 m) o Florisphaera profunda que prolifera en la zona fótica inferior (ZFI) (Winter et al., 1994). A este hecho hay que añadir que la profundidad de la capa fótica puede variar latitudinalmente, siendo más estrecha en latitudes altas y pudiendo llegar hasta los 220 metros de profundidad en latitudes bajas.

Tabla 2.2. Distribución esquemática general de los nutrientes, la luz y la temperatura en las diferentes zonas de la capa fótica en una región subtropical o tropical. Modificado de Jordan (2011).

\begin{tabular}{c||c|c|c}
\hline $\begin{array}{c}\text { Zona de la } \\
\text { capa fótica }\end{array}$ & $\begin{array}{c}\text { Concentración } \\
\text { de nutrientes }\end{array}$ & Luz & Temperatura \\
\hline \hline ZFS & Baja & & Alta \\
$(\mathbf{0 - 8 0} \mathbf{~ m})$ & $\left(<1 \mu \mathrm{mol}^{-1}\right)$ & $(100 \%)$ & $\left(>20^{\circ} \mathrm{C}\right)$ \\
\hline ZFM & Mayor que en la & Menor que en la & Más fría que la \\
$(\mathbf{8 0 - 1 2 0 ~})$ & ZFS & ZFS & ZFS \\
\hline ZFI & Alta & Baja & $\begin{array}{c}\text { Fría } \\
\left(<20^{\circ} \mathrm{C}\right)\end{array}$ \\
\hline$(\mathbf{1 2 0 - 2 0 0 ~})$ & $\left(>1 \mu \mathrm{mol}^{-1}\right)$ & $(<1 \%)$ &
\end{tabular}

\subsection{4.-Variabilidad espacial y temporal de la distribución de los cocolitóforos}

Además de todos los factores descritos anteriormente (luz, concentración de nutrientes, profundidad de la termoclina, etc.), otros como salinidad, corrientes costeras, estructuras de mesoescala, descargas fluviales y afloramientos afectan a la distribución biogeográfica de los cocolitóforos (Baumann et al., 2005; Guerreiro et al., 2013). Por este motivo ésta presenta una alta variabilidad espacial y temporal, de manera que la distribución y abundancia de cocolitóforos pertenecientes a una misma zona biogeográfica (Figura 2.6) puede variar entre océanos (Figura 2.7) y estaciones (Figura 2.8). De esta manera, la abundancia relativa de cocolitóforos en relación al total de fitoplancton (Figura 2.7) es mayor en el Atlántico norte y menor en el Atlántico ecuatorial y en el océano Índico (Gregg y Casey 2007). Respecto a la variación estacional, la distribución espacial de cocolitóforos (Figura 2.8) en invierno presenta mayores abundancias en las costas occidentales del océano Pacífico, mientras que en verano los máximos se registran en la región noratlántica (Gregg et al., 2007). 


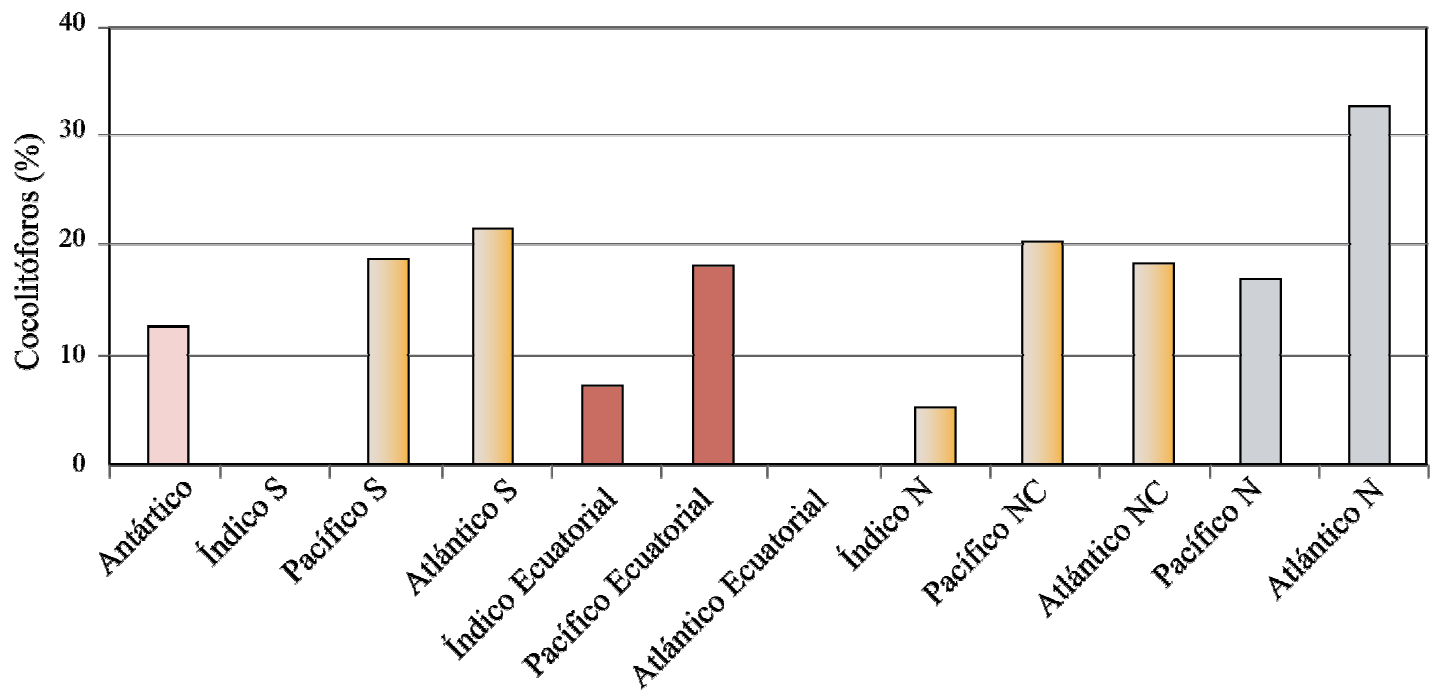

Figura 2.7. Abundancia relativa (\%) de cocolitóforos en los mares actuales en relación al total de fitoplancton. Modificada de Gregg y Casey (2007). Los colores de las barras se corresponden con la leyenda de la figura 2.6. Las barras bicolores representan zonas marítimas situadas entre las zonas templada y subtropical. S: sur; N: norte; NC: centro norte.
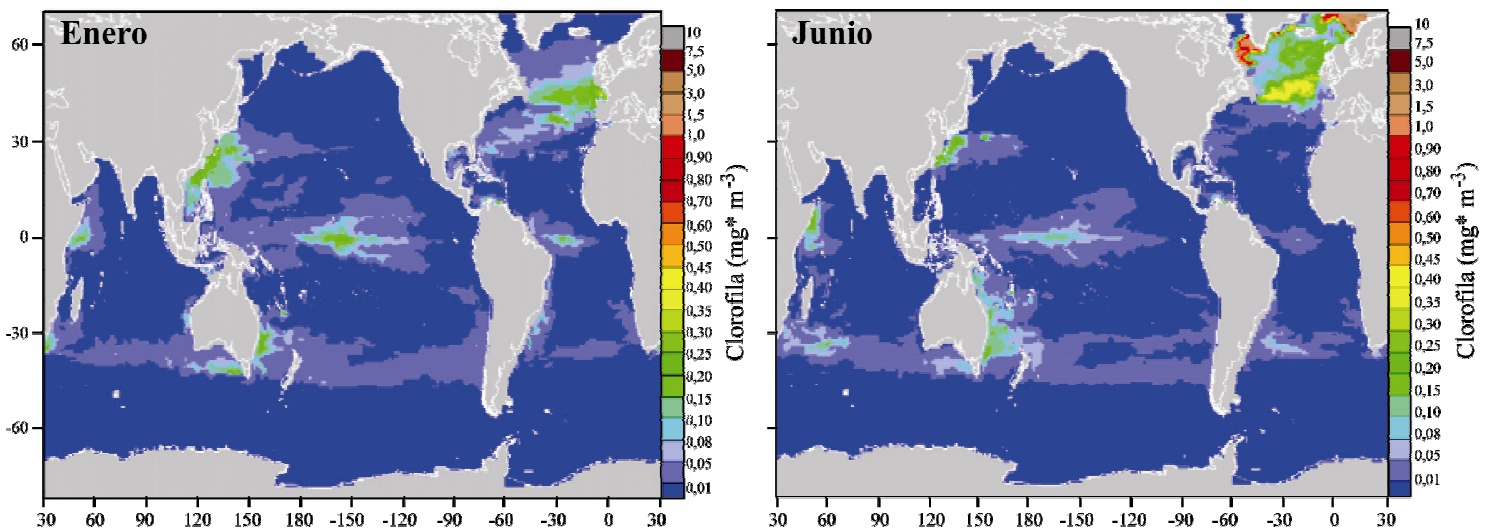

Figura 2.8. Distribución espacial de cocolitóforos en enero (izquierda) y en junio (derecha) medida en unidades de clorofila $\left(\mathrm{mg}^{*} \mathrm{~m}^{-3}\right)$. Modificada de Gregg et al. (2007).

\section{5.-DE LA ZONA FÓTICA AL REGISTRO FÓSIL:}

\subsection{1.-Transporte}

Cuando los cocolitóforos mueren lo más habitual es que la matriz orgánica asociada a los cocolitos se desintegre. La cocosfera entonces se disgrega y los cocolitos comienzan su transporte a lo largo de la columna de agua hasta los fondos oceánicos (Figura 2.9).

Debido a su geometría plana y su reducida masa, la tasa de hundimiento de los cocolitos es muy pequeña, aproximadamente $1 \mathrm{~m}^{*}$ día $^{-1}$ (Patara et al., 2009). Durante el tiempo que dure su hundimiento son sensibles a diversos procesos determinantes para su sedimentación y preservación. Entre ellos destacan la disolución de su calcita, su fragmentación por la actividad 
del zooplancton, los procesos de advección o transporte lateral por corrientes y los procesos de afloramiento o hundimiento de las masas de agua que los contienen (Patara et al., 2009).

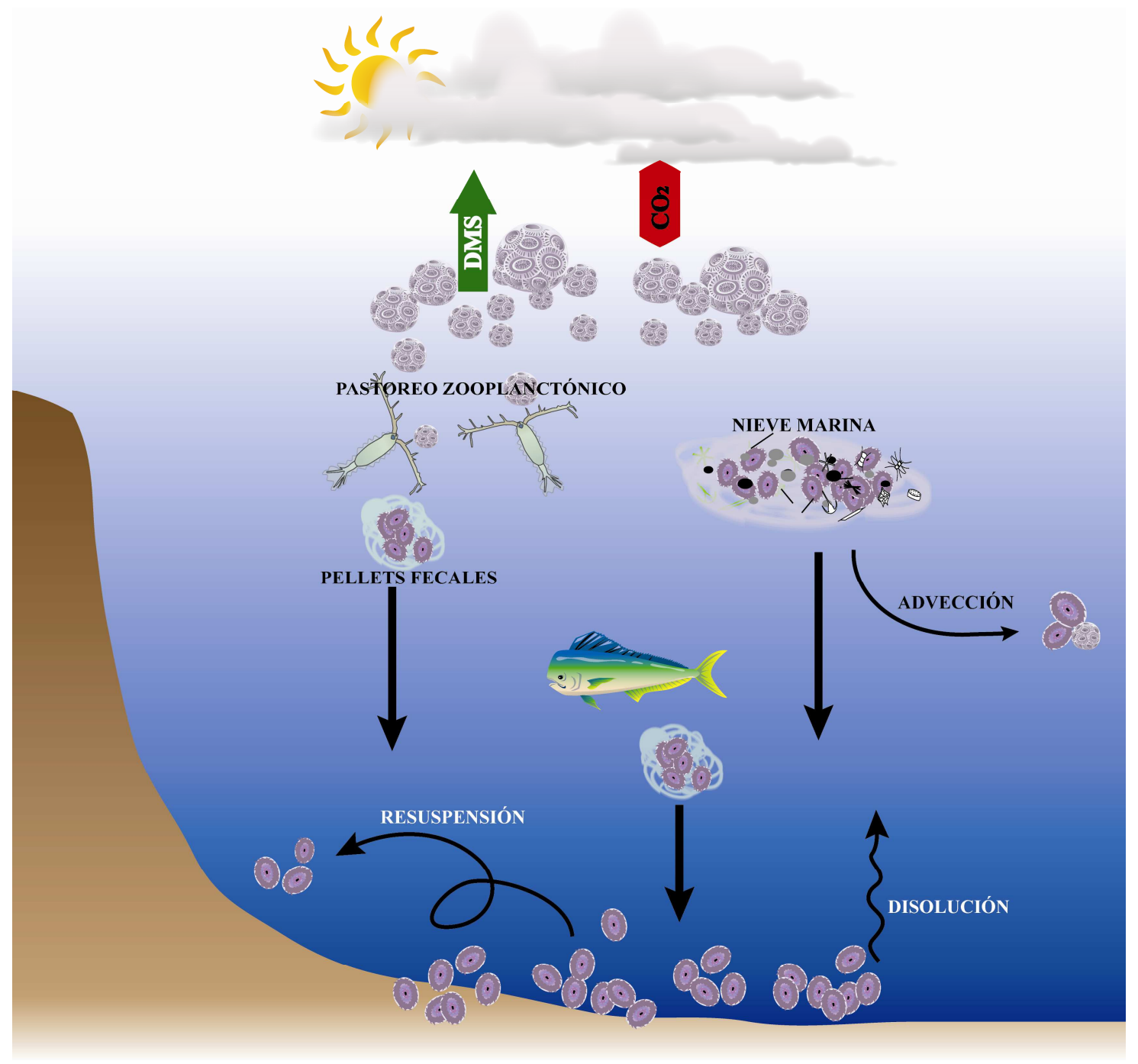

Figura 2.9. Procesos implicados en la producción, transporte, sedimentación y preservación de los cocolitóforos y cocolitos. DMS: dimetilsurfuro; $\mathrm{CO}_{2}$ : dióxido de carbono.

Sin embargo la mayoría de los cocolitos no se hunden de forma individual, sino que son incorporados a otros agregados de varios centímetros de tamaño como los pellets o aglomerados fecales producidos por el zooplancton o las partículas de nieve marina (Turner, 2002). Se ha observado que estos agregados pueden contener hasta $10^{5}$ cocolitos, acelerando la tasa de hundimiento de los mismos hasta los 162,5 m* día ${ }^{-1}$ (Honjo, 1976; Knappertsbusch y Brummer, 1995) y reduciendo sensiblemente el tiempo de exposición a otros procesos como la disolución. 


\subsection{2.-Sedimentación}

Las elevadas velocidades verticales a las que se hunden los pellets fecales y la nieve marina también minimizan los efectos del transporte lateral, asegurando que los cocolitos que sedimentan en una determinada zona tengan su origen en la comunidad fótica superficial correspondiente a dicha zona (Okada y Honjo, 1973; Baumann et al., 2000).

La sedimentación de cocolitos en los fondos marinos contribuye a mantener el gradiente de presión parcial de $\mathrm{CO}_{2}$ entre la superficie oceánica y las capas profundas, jugando así un papel determinante en la capacidad del océano para absorber $\mathrm{CO}_{2}$ atmosférico.

\subsection{3.-Preservación}

Tras su sedimentación, los cocolitos aún son sensibles a numerosos procesos que ocurren próximos a la interfase sedimento-agua y que afectarán a su adecuada preservación. Entre los más comunes cabe mencionar su disolución debida a la relación entre la acumulación de carbonato cálcico y el estado de saturación del agua, la resuspensión de sedimentos o la bioturbación. Cuanto mayor sea el flujo de partículas hacia las zonas profundas mayor será la protección de los cocolitos ante los procesos mencionados más arriba. Esto facilita su preservación y posterior conservación y fosilización, gracias a la cual se han recuperado cocolitos pertenecientes al Triásico superior (hace 200 millones de años) (Bown et al., 2004). 


\section{6.-SISTEMÁTICA DE LOS TAXONES ESTUDIADOS}

\begin{tabular}{|c|c|}
\hline División HAPTOPHYTA & Hibberd 1972 ex Edvardsen y Eikrem 2000 \\
\hline Clase PRYMNESIOPHYCEAE & Hibberd 1976; emend. Cavalier- Smith et al., 1996 \\
\hline Orden COCCOLITHALES & Schwar, 1932 sensu Jordan et al, 2004 \\
\hline Familia CALCIDISCACEAE & Young y Bown, 199 ? \\
\hline Gênero Calcidiscus & Kampter 1950 \\
\hline Especie Calcidiscus leptoporus & (Murray y Blackman, 1898) Loeblich y Tappan, 1978 \\
\hline Gẻnero Oolithotus & Reinhardt, 1968 \\
\hline Especie Oolithotus fragilis & (Lohmann, 1912) Martini y Müller, 1972 \\
\hline Género Umbilicosphaera & Lohmann, 1902 \\
\hline Especie Umbilicasphaera sibogae & (Weber- van Bosse, 1901) Gaarder, 1970 \\
\hline Familia COCCOLITHACEAE & Poche, 1913; emend. Young y Bown, 1997 \\
\hline Género Coccolithus & Schward, 1894 \\
\hline Especie Coccolithus pelagicus & (Wallich, 1877) Schiller, 1930 \\
\hline Subespecie Coccolithus pelagicus pelagicus & (Wallich 1877) Schiller 1930 \\
\hline Subespecie Coccolithus pelagicus braarudit & (Gaarder 1962) Geisen et al. 2002 \\
\hline Orden ISOCHRYSIDALES & Pascher, 1910 \\
\hline Familia NOELAERHABDACEAE & Jerkovic, 1970; emend. Young y Bown, 1997 \\
\hline Género Emiliania & Hay y Mohler, 1967 \\
\hline Especie Emiliania huxleyi & (Lohmann, 1902) Hay y Mohler, 1967 \\
\hline Género Gephyrocapsa & Kamptner, 1943 \\
\hline Especie Gephyrocapsa caribbeanica & Boudreaux and Hay, 1967 \\
\hline Especie Gephyrocapsa muellerae & Bréhéret, 1978 \\
\hline Especie Gephyracapsa oceúnica & Kamptner, 1943 \\
\hline \multicolumn{2}{|l|}{ Grupo "small" Gephyrocapsa } \\
\hline G. aperta & Bréhéret, 1978 \\
\hline G. ericsonii & Brêhéret, 1978 \\
\hline
\end{tabular}

\begin{tabular}{|c|c|}
\hline Orden SYRACOSPHAERALES & Hay, 1977; emend. Young et al., 2003 \\
\hline Familia SYRACOSPHAERACEAE & (Lohmann, 1902) Lemmermann, 1908 \\
\hline Género Syracosphaera & Lohmann, 1902 \\
\hline Especie Syracosphaera pulchra & Lohmann, 1902 \\
\hline Familia CALCIOSOLENIACEAE & Kampter, 1927 \\
\hline Género Calciosolenia & Gran, 1912; emend. Young et al., 2003 \\
\hline Especie Calciosolenia murrayi & Gran, 1912 \\
\hline Familia RHADOSPHAERACEAE & Haeckel, 1894 \\
\hline Gẻnero Rhabdosphaera & Haeckel, 1894 \\
\hline Especie Rhabdosphaera clavigera & Murray y Blackman, 1898 \\
\hline Orden ZYGODISCALES & Young y Bown, 1997 \\
\hline Familia HELICOSPHAERACEAE & Black, 1971 \\
\hline Género Helicasphaera & Kamptner, 1954 \\
\hline Especie Helicosphaera carteri & (Wallich, 1877) Kamptner, 1954 \\
\hline Familia PONTOSPHAERACEAE & Lemmermann, 1908 \\
\hline Género Pontosphaera & Lohmann, 1902 \\
\hline Especie Pontosphaera multipora (Kampter, 1984) & Roth, 1970 \\
\hline \multicolumn{2}{|l|}{ Grupo de nanolitos } \\
\hline FamiliaBRAARUDOSPHAERACEAE & Deflande 1947 \\
\hline Género Braarudosphaera & Deflandre 1947 \\
\hline Especie Braarudosphaera bigelowii & (Gran \& Braarud 1935) Deflandre 1947 \\
\hline Familia UMBELLOSPHAERGEAE & Young y Kleijne, 2003 \\
\hline Género Umbellosphaera & Paasche, 1955 \\
\hline Especie Umbellosphaera irregularis & Paasche, 1955 \\
\hline Especie Umbellosphaera temuis & (Kamptner, 1937) Paasche, 1955 \\
\hline \multicolumn{2}{|l|}{ Genera Incertae Sedis } \\
\hline Género Florisphaera & Okada y Honjo, 1973 \\
\hline Especie Florisphaera profunda & Okada y Honjo, 1973 \\
\hline
\end{tabular}




\section{7.-CARACTERÍSTICAS Y PREFERENCIAS ECOLÓGICAS DE LOS TAXONES ESTUDIADOS}

A excepción de los especímenes retrabajados, todas las especies cocolitoforales identificadas en esta tesis doctoral tienen representación en los océanos actuales. Este hecho presenta la ventaja de que sus requerimientos ecológicos pueden estudiarse mediante el análisis de muestras de trampas de sedimento y de la columna de agua. Esta información es de gran utilidad a la hora de inferir la variabilidad de las condiciones ambientales a lo largo de los últimos 25 ka a partir del estudio de las asociaciones cocolitoforales fósiles. A continuación se describen las características principales de los cocolitos de las especies consideradas en esta tesis doctoral así como sus requerimientos ecológicos.

\section{Género Calcidiscus Kampter 1950}

Calcidiscus leptoporus (Murray y Blackman, 1898) Loeblich y Tappan, 1978

Calcidiscus leptoporus (Anexo I, lámina 1) presenta tres morfotipos (Knappertsbusch et al., 1997), aunque Geisen et al. (2002) los considera como tres subespecies y otros autores (Young et al., 2003; Quinn et al., 2004) como tres especies diferentes. Estos tres morfotipos se distinguen por su tamaño y el número de elementos de su escudo distal. El morfotipo de mayor tamaño $(7-11 \mu \mathrm{m})$ tiene entre 20 y 35 elementos, el morfotipo mediano $(5-8 \mu \mathrm{m})$ tiene entre 15 y 30 elementos y el morfotipo pequeño $(3-5 \mu \mathrm{m})$ presenta entre 10 y 20 elementos. Se trata de una especie robusta menos sensible a la disolución selectiva. Clásicamente ha sido considerada como una especie de aguas oligotróficas y distribución global (Giraudeau y Rogers, 1994; Ziveri et al., 2004), si bien es cierto que sus requerimientos ecológicos específicos son poco conocidos. Ha sido relacionada con aguas cálidas $\left(20-30{ }^{\circ} \mathrm{C}\right)$ (Okada y Honjo, 1973) y también frías $\left(6{ }^{\circ} \mathrm{C}\right)$ (McIntyre et al., 1970), por lo que podría considerarse euriterma. En el Mediterráneo occidental ha sido caracterizada como una especie de aguas oligotróficas (Colmenero-Hidalgo et al., 2004) aunque otros autores (Giraudeau, 1992; Flores et al., 1997) lo relacionan con la disponibilidad de nutrientes.

Género Oolithotus Reinhardt, 1968

Oolithotus fragilis (Lohmann, 1912) Martini y Müller, 1972

Oolithotus fragilis (Anexo I, lámina 2) se caracteriza por cocolitos de tamaño entre 6,7 y 8,7 $\mu \mathrm{m}$ (Cros y Fortuño, 2002). Bajo el microscopio óptico puede confundirse con C. leptoporus, aunque se diferencia de este último en que su tubo y escudo proximal están más alejados del centro del cocolito. Es una de las pocas especies que habitan la zona fótica intermedia o inferior (Young, 1994) y es considerada como indicadora de aguas oligotróficas (Brand, 1994). 


\section{Género Umbilicosphaera Lohmann, 1902}

Umbilicosphaera sibogae (Weber-van Bosse, 1901) Gaarder, 1970

Los cocolitos de esta Umbilicosphaera sibogae (Anexo I, lámina 3) tienen forma circular y una gran abertura central. Su tamaño varía entre 3 y $7 \mu \mathrm{m}$ (Cros y Fortuño, 2002). La distribución de $U$. sibogae se restringe a latitudes subtropicales y tropicales y aguas templadas $\left(>15^{\circ} \mathrm{C}\right)$ y pobres en nutrientes (Okada y Mclntyre, 1979; Giraudeau, 1992; Brand, 1994). En el Mediterráneo occidental es considerada como un taxón cálido (Flores et al., 1997).

Género Coccolithus Schward, 1894

Coccolithus pelagicus (Wallich, 1877) Schiller, 1930

Esta especie puede encontrarse en la literatura como un indicador clásico de aguas frías (McIntyre y Bé, 1967; Okada y Mclntyre, 1979; Winter et al., 1994) pero también de regiones de afloramiento (Cachão y Moita, 2000). Sus preferencias ecológicas se definieron más adelante con la existencia de varias subespecies de C. pelagicus que pueden diferenciarse por sus ciclos de vida (Geisen et al., 2002), genética (Sáez et al., 2003) y el tamaño de sus placolitos (Parente et al., 2004).

Coccolithus pelagicus subsp. pelagicus (Wallich, 1877) Schiller, 1930

Coccolithus pelagicus subsp. pelagicus (Anexo I, lámina 4) presenta cocolitos de entre 7 y $10 \mu \mathrm{m}$ de tamaño y gran robustez, lo que les confiere un mayor potencial de preservación en el registro fósil (Cachão y Moita, 2000). Es una forma característica de la región subártica (Geisen et al., 2002) y es utilizado como indicador de aguas frías (Narciso et al., 2006; Amore et al., 2012).

Coccolithus pelagicus subsp. braarudii (Gaarder 1962) Geisen, 2002

Los cocolitos de Coccolithus pelagicus subsp. braarudii (Anexo I, lámina 5) presentan un mayor tamaño que la subespecie pelagicus $(10-16 \mu \mathrm{m})$ y ha sido relacionado con aguas más templadas de frentes moderados dirigidos por condiciones de afloramiento (Cachão y Moita, 2000; Amore et al., 2012). 
Género Emiliania Hay y Mohler, 1967

Emiliania huxleyi (Lohmann, 1902) Hay y Mohler, 1967

La especie Emiliania huxleyi (Anexo I, lámina 6) presenta cocolitos de pequeño tamaño (2-4 $\mu \mathrm{m})$ que se caracterizan por la ausencia de puente. E. huxleyi podría considerarse la especie actual más abundante en los sedimentos oceánicos recientes alcanzando abundancias relativas de hasta el $80 \%$ (Winter et al., 1994). Se trata de una especie cosmopolita capaz de proliferar en un variado espectro de condiciones ambientales y amplios rangos de temperatura $\left(1-30{ }^{\circ} \mathrm{C}\right)$ (Okada y Mclntyre, 1979), salinidad (11-41 ppt) (Buckry, 1974; Winter et al., 1979) y nutrientes (Roth, 1994; Winter et al., 1994). Es considerada como una estratega r, de forma que aprovecha la disponibilidad de nutrientes para proliferar en gran número, alcanzando hasta $10^{6}$ células por litro.

Los estudios biométricos sobre las asociaciones fósiles del Atlántico norte revelan la existencia de dos morfotipos que se distinguen por su tamaño: uno con un eje mayor de tamaño $<4 \mu \mathrm{m}$ (Anexo I, Lámina 6) y otro morfotipo con un eje mayor de tamaño $>4 \mu \mathrm{m}$ (Anexo I, lámina 7) (Pujos-Lamy, 1977; Weaver et al., 1999; Flores et al., 2010). Los estudios realizados en el Mediterráneo occidental (Colmenero-Hidalgo et al., 2002; Colmenero-Hidalgo et al., 2004) asocian el morfotipo de mayor tamaño a la entrada de agua fría atlántica en el Mediterráneo.

E. huxleyi es considerada, junto a Gephyrocapsa oceanica, como la mayor productora de alquenonas (Conte et al., 1998), un compuesto orgánico que depende de la temperatura superficial oceánica (Müller et al., 1998).

\section{Género Gephyrocapsa Kamptner, 1943}

Las especies pertenecientes a este género se caracterizan por cocolitos de tamaño pequeño o intermedio cuya área central es atravesada por un puente de calcita. Para la clasificación de las especies pertenecientes a este género se ha seguido la clasificación propuesta por Flores et al. (1997), modificada posteriormente por Colmenero-Hidalgo et al. (2004).

Género Gephyrocapsa Kamptner, 1943

Gephyrocapsa caribbeanica Boudreaux and Hay, 1967

Los cocolitos de la especie Gephyrocapsa caribbeanica (Anexo I, lámina 8), robustos y ligeramente redondeados, presentan un eje mayor superior a $3 \mu \mathrm{m}$. Presentan el área central parcial o totalmente cerrada y su puente forma un ángulo $<25^{\circ}$ con el eje mayor del cocolito. Se ha observado que esta especie aumenta su abundancia durante los periodos fríos del hemisferio 
sur (Okada y Wells, 1997; Flores et al., 1999). Sin embargo, Flores et al. (2000) registraron un descenso de la misma durante el último estadio glacial frente a las costas de África occidental.

Género Gephyrocapsa Kamptner, 1943

Gephyrocapsa muellerae Bréhéret, 1978

Los cocolitos de la especie Gephyrocapsa muellerae (Anexo I, lámina 9) presentan un eje mayor superior a $3 \mu \mathrm{m}$ y muestran una forma ligeramente más ovalada y un puente menos robusto que el de otras especies del género Gephyrocapsa. El puente forma un ángulo $<25^{\circ}$ con el eje mayor del placolito. Esta especie es considerada un indicador de aguas más frías (Weaver y Pujol, 1988; Ziveri et al., 2004) aunque los estudios a partir de trampas de sedimento en el Mediterráneo occidental muestran que G. muellerae también responde al afloramiento de aguas frías y ricas en nutrientes (Bárcena et al., 2004; Hernández-Molina et al., 2011).

Género Gephyrocapsa Kamptner, 1943

Gephyrocapsa oceanica Kamptner, 1943

La especie Gephyrocapsa oceanica (Anexo I, lámina 10) presenta cocolitos robustos, ligeramente redondeados y con un eje mayor normalmente superior a $3 \mu \mathrm{m}$. El puente forma un ángulo $>50^{\circ}$ con el eje mayor del cocolito. Muestra afinidad por aguas eutróficas (Giraudeau, 1992; Ziveri et al., 1995) y una amplia distribución en los océanos actuales, desde aguas templadas hasta tropicales y de mares marginales (McIntyre y Bé, 1967; Okada y Honjo, 1973; Okada y Mclntyre, 1979). En el Mediterráneo occidental ha sido ligada a aguas cálidas (Weaver y Pujol, 1988; Flores et al., 1999). Otros autores sin embargo la han relacionado con la entrada de aguas atlánticas de menor salinidad (Knappertsbusch, 1993; Cros, 1995; Bárcena et al., 2004).

Género Gephyrocapsa Kamptner, 1943

\section{Grupo "small” Gephyrocapsa}

El grupo "small" Gephyrocapsa incluye aquellas especies del género Gephyrocapsa que presenten cocolitos de pequeño tamaño (eje mayor $<3 \mu \mathrm{m}$ ) (Anexo I, lámina 11) por lo que su identificación con el microscopio óptico es complicada. Normalmente el grupo "small" Gephyrocapsa contendrá más de una especie, siendo las más comunes G. aperta y G. ericsonii. La disolución selectiva puede afectar a su puente pudiendo llegar a confundirse con E. huxleyi. Sin embargo, este hecho no tiene mayores consecuencias para los estudios paleoecológicos, ya que este grupo suele aflorar junto a E. huxleyi, de naturaleza igualmente oportunista, 
contemplándose todas ellas bajo el término "pequeños placolitos" y utilizándose como indicadoras de aguas ricas en nutrientes o de alta productividad (Colmenero-Hidalgo et al., 2004; Maiorano et al., 2009; Amore et al., 2012).

\section{Género Syracosphaera Lohmann, 1902}

Éste es uno de los géneros de cocolitóforos con mayor diversidad de especies (Giraudeau, 1992). Su identificación a nivel de especie con microscopía óptica es complicada y su biogeografía no presenta un patrón definido (Okada y Mclntyre, 1979).

Género Syracosphaera Lohmann, 1902

Syracosphaera pulchra Lohmann, 1902

La especie Syracosphaera pulchra (Anexo I, lámina 12) es considerada como la más común del género Syracosphaera. Sus cocolitos presentan forma ovalada y un tamaño $>5 \mu \mathrm{m}$. Su distribución abarca los $50^{\circ} \mathrm{N}$ y los $50^{\circ} \mathrm{S}$ (Ziveri et al., 1995; Ziveri et al., 2004) y no presenta una clara preferencia por una zona concreta de la capa fótica (Flores et al., 1999). Sus preferencias ecológicas no están bien definidas, aunque ha sido relacionada con aguas pobres en nutrientes (Ziveri et al., 2004) o con aguas de baja salinidad (Buckry, 1974; Weaver y Pujol, 1988; Colmenero-Hidalgo et al., 2004).

Género Calciosolenia Gran, 1912; emend. Young et al., 2003

\section{Calciosolenia murrayi Gran, 1912}

La especie Calciosolenia murrayi (Anexo I, lámina 13) es de muy fácil identificación ya que sus cocolitos presentan una forma rómbica alargada muy particular, cuyo eje mayor mide entre 3 y $4 \mu \mathrm{m}$. Sin embargo, hay que tener en cuenta que puede llegar a extinguirse a la luz polarizada lineal del microscopio óptico, por lo que es recomendable rotar la platina del mismo. Es habitual encontrarla en aguas tropicales y subtropicales por lo que es incluida entre los taxones cálidos (Okada y Mclntyre, 1977).

\section{Género Rhabdosphaera Haeckel, 1894}

Rhabdosphaera clavigera Murray y Blackman, 1898

Los cocolitos de Rhabdosphaera clavigera (Anexo I, lámina 14) se caracterizan por la presencia de una espina muy desarrollada en forma de bastón compuesta por listones alargados. 
$R$. clavigera es característica de aguas subtropicales, templadas o cálidas $\left(14-30{ }^{\circ} \mathrm{C}\right)$, salinas y oligotróficas (Okada y Honjo, 1973; Geitzenauer et al., 1976; Roth, 1994).

Género Helicosphaera Kamptner, 1954

Helicosphaera carteri (Wallich, 1877) Kamptner, 1954

H. carteri (Anexo I, lámina 15) es una de las especies más comunes de este género. Sus cocolitos son grandes (eje mayor entre 6 y $12 \mu \mathrm{m}$ ), ovalados y robustos. Es elíptica y en ocasiones asimétrica, presentando un ala en alguno de sus extremos y dos pequeñas hendiduras en su área central. Es una especie cosmopolita que tolera un amplio rango de temperaturas (5-30 ${ }^{\circ} \mathrm{C}$ ) con un óptimo de $21^{\circ} \mathrm{C}$ en aguas templadas (McIntyre y Bé, 1967; Okada y Mclntyre, 1979). Algunos autores la han relacionado con bajas concentraciones de nutrientes y turbidez en la capa fótica (Brand, 1994; Ziveri et al., 1995), mientras que otros la han encontrado en regiones de afloramiento (Estrada, 1978; Giraudeau, 1992) o la han correlacionado con periodos altamente productivos en sedimentos del plio-pleistoceno (Pujos, 1992; Flores et al., 1995). Su aparición en el Mediterráneo occidental junto a $S$. pulchra ha sido interpretada como un indicador de baja salinidad (Colmenero-Hidalgo et al., 2004).

Género Pontosphaera Lohmann, 1902

Las especies pertenecientes a este género suelen aparecer en regiones tropicales o subtropicales, en zonas oligotróficas y cálidas (Okada y Mclntyre, 1977).

Género Pontosphaera Lohmann, 1902

Pontosphaera multipora (Kampter, 1984) Roth, 1970

Los cocolitos de Pontosphaera multipora (Anexo I, lámina 16) son grandes (eje mayor $>9$ $\mu \mathrm{m})$, de forma ovalada con un borde bien definido y elevado y presentan entre $10 \mathrm{y} 44$ poros.

\section{Género Braarudosphaera Deflandre 1947}

\section{Braarudosphaera bigelowii (Gran \& Braarud 1935) Deflandre 1947}

Los cocolitos de Braarudosphaera bigelowii (Anexo I, lámina 17) están formados por una única unidad cristalina que presenta simetría radial y forma pentagonal de entre 3 y $7 \mu \mathrm{m}$.

Esta especie tolera un amplio rango de salinidades pudiendo habitar en condiciones ambientales extremas o inusuales (Roth, 1994), como confirma su presencia en sedimentos 
depositados inmediatamente después de las extinciones correspondientes al límite $\mathrm{K} / \mathrm{T}$ (PerchNielsen, 1985). La mayoría de los estudios sugieren preferencia por ambientes de baja salinidad y alta turbidez causada por la entrada de terrígenos (Buckry, 1974; Roth, 1994). En sedimentos cretácicos y paleógenos presenta altas abundancias llegando incluso a dominar la asociación, lo cual ha sido interpretado como condiciones de afloramiento de aguas frías, poco salinas y ricas en nutrientes y de baja competición con otras especies (Peleo-Alampay et al., 1999; Švabenicka, 1999).

Género Umbellosphaera Paasche, 1955

Umbellosphaera irregularis Paasche, 1955

Los cocolitos de Umbellosphaera irregularis (Anexo I, lámina 18) tienen forma de trompeta, son cóncavos y su superficie distal es lisa. Esta especie es considerada un indicador de aguas cálidas con una temperatura superior a los $25^{\circ} \mathrm{C}$ (Winter et al., 1994).

Género Umbellosphaera Paasche, 1955

Umbellosphaera tenuis (Kamptner, 1937) Paasche, 1955

Los cocolitos de esta especie también tienen forma de trompeta, pero son convexos y su superficie distal aparece ornamentada. Es común en latitudes subtropicales en la capa fótica intermedia y muestra preferencia por aguas más templadas que $U$. irregularis (entre 14 y $21^{\circ} \mathrm{C}$ ) (McIntyre et al., 1970).

Género Florisphaera Okada y Honjo, 1973

Florisphaera profunda Okada y Honjo, 1973

Los cocolitos de Florisphaera profunda (Anexo I, lámina 19) tienen forma de placa trapezoidal ligeramente curvada. Presentan baja birrefringencia aunque por su forma pueden confundirse con arcillas. Sin embargo, y como ocurría con los cocolitos de C. murrayi, pueden extinguirse bajo la luz polarizada lineal del microscopio óptico, por lo que es recomendable girar la platina del mismo para su correcta identificación. Es una de las pocas especies cocolitoforales que habitan la capa fótica inferior, entre los 120-220 metros de profundidad, entre los $40^{\circ} \mathrm{N}$ y $40^{\circ} \mathrm{S}$ (Okada y Honjo, 1973; Okada y Wells, 1997). F. profunda presenta una preferencia clara por bajas intensidades lumínicas y escasa turbidez (Ahagon et al., 1993; Ziveri et al., 1995). Se considera un buen indicador de la profundidad de la nutriclina viendo aumentada su abundancia respecto a la de otros taxones de la capa fótica superior cuando la 
nutriclina es más profunda, la columna de agua es estable y la mezcla vertical escasa (Molfino y McIntyre, 1990; Beaufort et al., 1997; Flores et al., 2000; Beaufort et al., 2001; Incarbona et al., 2008).

\section{Referencias}

Ahagon N, Tanaka Y y Ujiié H. (1993) Florisphaera profunda, a possible nannoplankton indicator of late Quaternary changes in sea-water turbidity at the northwestern margin of the Pacific. Marine Micropaleontology 22: 255-273.

Amore FO, Flores JA, Voelker AHL, Lebreiro SM, Palumbo E y Sierro FJ. (2012) A Middle Pleistocene Northeast Atlantic coccolithophore record: Paleoclimatology and paleoproductivity aspects. Marine Micropaleontology 90-91: 44-59.

Bárcena MA, Flores JA, Sierro FJ, Pérez-Folgado M, Fabres J, Calafat A y Canals M. (2004) Planktonic response to main oceanographic changes in the Alboran Sea (Western Mediterranean) as documented in sediment traps and surface sediments. Marine Micropaleontology 53: 423-445.

Baumann KH, Andruleit H, Boeckel B, Geisen M y Kinkel H. (2005) The significance of extant coccolithophores as indicators of ocean water masses, surface water temperature, and palaeoproductivity: a review. Palaeontologische Zeitschrift 79: 93-112.

Baumann KH, Andruleit H y Samtleben C. (2000) Coccolithophores in the Nordic Seas: comparison of living communities with surface sediment assemblages. Deep Sea Research Part II: Topical Studies in Oceanography 47: 1743-1772.

Beaufort L, de Garidel-Thoron T, Mix AC y Pisias NG. (2001) ENSO-like Forcing on Oceanic Primary Production During the Late Pleistocene. Science 293: 2440-2444.

Beaufort L, Lancelot Y, Camberlin P, Cayre O, Vincent E, Bassinot F y Labeyrie L. (1997) Insolation Cycles as a Major Control of Equatorial Indian Ocean Primary Production. Science 278: 1451-1454.

Billard C y Inouye I. (2004) What is new in coccolithophore biology? En: Thierstein HR y Young JR (eds) Coccolithophores: from molecular processes to global impact. Springer Berlin Heidelberg, 1-29.

Bown PR, Lees JA y Young JR. (2004) Calcareous nannoplankton evolution and diversity through time. En: Thierstein HR y Young JR (eds) Coccolithophores: from molecular processes to global impact. Springer Berlin Heidelberg, 481-508.

Brand LE. (1994) Physiological ecology of marine coccolithophores. En: Winter A y Siesser WG (eds) Coccolithophores. Cambridge: Cambridge University Press, 33-49.

Buckry D. (1974) Coccoliths as paleosalinity indicators-evidence from the Black Sea. Memoirs of the America Association of Petroleum Geologists 20: 353-363. 
Cachão M y Moita MT. (2000) Coccolithus pelagicus, a productivity proxy related to moderate fronts off Western Iberia. Marine Micropaleontology 39: 131-155.

Colmenero-Hidalgo E, Flores JA y Sierro FJ. (2002) Biometry of Emiliania huxleyi and its biostratigraphic significance in the Eastern North Atlantic Ocean and Western Mediterranean Sea in the last 20,000 years. Marine Micropaleontology 46: 247-263.

Colmenero-Hidalgo E, Flores JA, Sierro FJ, Bárcena MÁ, Löwemark L, Schönfeld J y Grimalt JO. (2004) Ocean surface water response to short-term climate changes revealed by coccolithophores from the Gulf of Cadiz (NE Atlantic) and Alboran Sea (W Mediterranean). Palaeogeography, Palaeoclimatology, Palaeoecology 205: 317-336.

Conte MH, Thompson A, Lesley D y Harris RP. (1998) Genetic and Physiological Influences on the Alkenone/Alkenoate Versus Growth Temperature Relationship in Emiliania huxleyi and Gephyrocapsa Oceanica. Geochimica et Cosmochimica Acta 62: 51-68.

Cros L. (1995) Calcareous nannoplankton in surficial sediments of the Catalano-Balearic Sea. (Northwestern Mediterranean). En: 5th INA Conference in Salamanca 1993. En: Flores JA y Sierro FJ (eds) Proceedings, Universidad de Salamanca. 47-59.

Cros L y Fortuño J-M. (2002) Atlas of northwestern Mediterranean coccolithophores. Scientia Marina 66: 7-182.

Estrada M. (1978) Mesoscale Heterogeneities of the Phytoplankton Distribution in the Upwelling Region of NW Africa. En: Boje R y Tomczak M (eds) The upwelling region on North West Africa (Upwelling Ecosystems). Berlin: Springer Berlin Heidelberg, 1523.

Flores JA, Bárcena MA y Sierro FJ. (2000) Ocean-surface and wind dynamics in the Atlantic Ocean off Northwest Africa during the last 140000 years. Palaeogeography, Palaeoclimatology, Palaeoecology 161: 459-478.

Flores JA, Colmenero-Hidalgo E, Mejía-Molina AE, Baumann KH, Henderiks J, Larsson K, Prabhu CN, Sierro FJ y Rodrigues T. (2010) Distribution of large Emiliania huxleyi in the Central and Northeast Atlantic as a tracer of surface ocean dynamics during the last 25,000 years. Marine Micropaleontology 76: 53-66.

Flores JA, Gersonde R y Sierro FJ. (1999) Pleistocene fluctuations in the Agulhas Current Retroflection based on the calcareous plankton record. Marine Micropaleontology 37: $1-22$.

Flores JA y Sierro FJ. (2007) Paleoceanography, biological proxies: Coccoliths. En: Scott EA (ed) Encyclopedia of Quaternary Science. Amsterdam: Eselvier, 1634-1647.

Flores JA, Sierro FJ, Francés G, Vázquez A y Zamarreño I. (1997) The last 100,000 years in the western Mediterranean: sea surface water and frontal dynamics as revealed by coccolithophores. Marine Micropaleontology 29: 351-366. 
Flores JA, Sierro FJ y Raffi I. (1995) Evolution of the calcareous nannofossil assemblage as a response to the paleoceanographic changes in the Eastern equatorial Pacific from 4 to 2 Ma (Leg 138, Sites 849 and 852). Proceedings of the Ocean Drilling Program, Scientific Results 138: 163-176.

Geisen M, Billard C, Broerse A, Cros L, Probert I y Young JR. (2002) Life-cycle associations involving pairs of holococcolithophorid species: intraspecific variation or cryptic speciation? European Journal of Phycology 37: 531-550.

Geitzenauer KR, Roche MB y McIntyre A. (1976) Modern Pacific coccolith assemblages: derivation and application to late Pleistocene paleotemperature analysis. Geological Society of America Memoir 145: 423-448.

Giraudeau J. (1992) Distribution of Recent nannofossils beneath the Benguela system: Southwest African continental margin. Marine Geology 108: 219-237.

Giraudeau J y Rogers J. (1994) Phytoplankton biomass and sea-surface temperature estimates from sea-bed distribution of nannofossils and planktonic foraminifera in the Benguela upwelling system. Micropaleontology 40: 275-285.

Green JC y Hori T. (1994) Flagella and flagellar roots. En: Green JC y Leadbeater BSC (eds) The Haptophyte Algae. Systematics Association. Oxford: Clarendon Press, 47-71.

Gregg WW y Casey NW. (2007) Modeling coccolithophores in the global oceans. Deep Sea Research Part II: Topical Studies in Oceanography 54: 447-477.

Guerreiro C, Oliveira A, de Stigter H, Cachão M, Sá C, Borges C, Cros L, Santos A, Fortuño JM y Rodrigues A. (2013) Late winter coccolithophore bloom off central Portugal in response to river discharge and upwelling. Continental Shelf Research 59: 65-83.

Hernández-Molina FJ, Serra N, Stow DAV, Llave E, Ercilla G y Van Rooij D. (2011) Alongslope oceanographic processes and sedimentary products around the Iberian margin. Geo-Marine Letters 31: 315-341.

Hibberd DJ. (1976) The ultrastructure and taxonomy of the Chrysophyceae and Prymnesiophyceae (Haptophyceae): survey with some new observations on the ultrastructure of the Chrysophyceae. Botanical Journal of the Linnean Society 72: 5580.

Honjo S. (1976) Coccoliths: Production, transportation and sedimentation. Marine Micropaleontology 1: 65-79.

Incarbona A, Di Stefano E, Patti B, Pelosi N, Bonomo S, Mazzola S, Sprovieri R, Tranchida G, Zgozi S y Bonanno A. (2008) Holocene millennial-scale productivity variations in the Sicily Channel (Mediterranean Sea). Paleoceanography 23: PA3204.

Jordan RW. (2011) Coccolithophores. En: Schaechter M (ed) Eukaryotic Microbes. San Diego, USA, 235-246. 
Jordan RW y Chamberlain AHL. (1997) Biodiversity among haptophyte algae. En: Hawksworth D (ed) Biodiversity and Conservation. 131-152.

Keller MD. (1989) Dimethyl Sulfide Production and Marine Phytoplankton: The Importance of Species Composition and Cell Size. Biological Oceanography 6: 375-382.

Kiermayer O y Romanovicz DK. (1981) Scale Formation in Flagellates. Cytomorphogenesis in Plants. Springer Vienna, 27-62.

Knappertsbusch M. (1993) Geographic distribution of living and Holocene coccolithophores in the Mediterranean Sea. Marine Micropaleontology 21: 219-247.

Knappertsbusch M y Brummer GJA. (1995) A sediment trap investigation of sinking coccolithophorids in the North Atlantic. Deep Sea Research Part I: Oceanographic Research Papers 42: 1083-1109.

Knappertsbusch M, Cortes MY y Thierstein HR. (1997) Morphologic variability of the coccolithophorid Calcidiscus leptoporus in the plankton, surface sediments and from the Early Pleistocene. Marine Micropaleontology 30: 293-317.

Lloyd GT, Smith AB y Young JR. (2011) Quantifying the deep-sea rock and fossil record bias using coccolithophores. Geological Society, London, Special Publications 358: 167177.

Maiorano P, Marino M y Flores JA. (2009) The warm interglacial Marine Isotope Stage 31: Evidences from the calcareous nannofossil assemblages at Site 1090 (Southern Ocean). Marine Micropaleontology 71: 166-175.

Malin G y Erst GO. (1997) Algal production of dimethyl sulfide and its atmospheric role. Journal of Phycology 33: 889-896.

Manton I. (1964) Further observations on the fine structure of the haptonema in prymnesium parvum. Archive Mikrobiologie 49: 315-330.

McIntyre A y Bé AWH. (1967) Modern coccolithophoridae of the Atlantic Ocean. Placoliths and cyrtoliths. Deep Sea Research and Oceanographic Abstracts 14: 561-597.

McIntyre A, Bé AWH y Roche MB. (1970) Modern Pacific coccolithophorida: a paleoontological thermometer Transactions of the New York Academy of Sciences 32: 720-731.

Molfino B y McIntyre A. (1990) Nutricline variation in the equatorial Atlantic coincident with the Younger Dryas. Paleoceanography 5: 997-1008.

Müller PJ, Kirst G, Ruhland G, von Storch I y Rosell-Melé A. (1998) Calibration of the alkenone paleotemperature index $\mathrm{U}_{37}{ }^{\mathrm{K}}$ based on core-tops from the eastern South Atlantic and the global ocean $\left(60^{\circ} \mathrm{N}-60^{\circ} \mathrm{S}\right)$. Geochimica et Cosmochimica Acta 62 : 1757-1772.

Narciso A, Cachão M y de Abreu L. (2006) Coccolithus pelagicus subsp. pelagicus versus Coccolithus pelagicus subsp. braarudii (Coccolithophore, Haptophyta): A proxy for 
surface subarctic Atlantic waters off Iberia during the last 200 kyr. Marine Micropaleontology 59: 15-34.

Okada H y Honjo S. (1973) The distribution of oceanic coccolithophorids in the Pacific. Deep Sea Research and Oceanographic Abstracts 20: 355-374.

Okada H y Mclntyre A. (1977) Modern coccolithophores of the Pacific and North Atlantic oceans. Micropaleontology 23: 1-55.

Okada H y Mclntyre A. (1979) Seasonal distribution of modern coccolithophores in the western North Atlantic Ocean. Marine Biology 54: 319-328.

Okada H y Wells P. (1997) Late Quaternary nannofossil indicators of climate change in two deep-sea cores associated with the Leeuwin Current off Western Australia. Palaeogeography, Palaeoclimatology, Palaeoecology 131: 413-432.

Paasche E. (2001) A review of the coccolithophorid Emiliania huxleyi (Prymnesiophyceae), with particular reference to growth, coccolith formation, and calcificationphotosynthesis interactions. Phycologia 40: 503-529.

Parente A, Cachão M, Baumann K-H, de Abreu L y Ferreira J. (2004) Morphometry of Coccolithus pelagicus s: 1. (Coccolithophore, Haptophyta) from offshore Portugal, during the last $200 \mathrm{kyr}$. Micropaleontology 50: 107-120.

Patara L, Pinardi N, Corselli C, Malinverno E, Tonani M, Santoleri R y Masina S. (2009) Particle fluxes in the deep Eastern Mediterranean basins: the role of ocean vertical velocities. Biogeosciences 6: 333-348.

Peleo-Alampay AM, Mead GA y Wei W. (1999) Unusual Oligocene Braarudosphaerarich layers of the South Atlantic and their paleooceanographic implications. Journal Nannoplankton Research 21: 17-26.

Perch-Nielsen K. (1985) Cenozoic calcareous nannofossils. En: Bolli HM, Saunders JB y PerchNielsen K (eds) Plankton Stratigraphy. Cambridge: Cambridge University Press, 427554.

Pienaar RN. (1994) Ultrastructure and calcification of coccolithophores. En: Winter A y Siesser W (eds) Coccolithophores. Cambridge: Cambridge University Press, 13-37.

Pujos-Lamy A. (1977) Emiliania et Gephyrocapsa (nannoplancton calcaire): biométrie et intérêt bostratigraphique dans le Pleistoncène Supérieur marin des Açores. Revista Española de Micropaleontología 9: 69-84.

Pujos A. (1992) Calcareous nannofossils of Plio-Pleistocene sediments from the northwestern margin of tropical Africa. Geological Society, London, Special Publications 64: 343358.

Quinn PS, Sáez AG, Baumann K-H, Steel BA, Sprengel C y Medlin LK. (2004) Coccolithophorid biodiversity: evidence from the cosmopolitan species Calcidiscus 
leptoporus. En: Thierstein HR y Young JR (eds) Coccolithophores: From Molecular Processes to Global Impact. Springer Berlin Heidelberg, 299-326.

Roth PH. (1994) Distribution of coccoliths in ocean sediments. En: Winter A y Siesser WG (eds) Coccolithophores. Cambridge: Cambridge University Press, 199-218.

Sáez AG, Probert I, Geisen M, Quinn P, Young JR y Medlin LK. (2003) Pseudo-cryptic speciation in coccolithophores. Proceedings of the National Academy of Sciences of the United States of America 100: 7163-7168.

Švabenicka L. (1999) Braarudosphaera-rich sediments in the Turonian of the Bohemian Cretaceous Basin, Czech Republic. Cretaceous Research 20: 773-782.

Thierstein H, Young J, Billard C y Inouye I. (2004) What is new in coccolithophore biology? Coccolithophores. Springer Berlin Heidelberg, 1-29.

Turner JT. (2002) Zooplankton fecal pellets, marine snow and sinking phytoplankton blooms. Aquatic Microbial Ecology 27: 57-102.

Tyrrell T, Young JR y Steele JH. (2009) Coccolithophores. Encyclopedia of Ocean Sciences (Second Edition). Oxford: Academic Press, 606-614.

Weaver PPE, Chapman MR, Eglinton G, Zhao M, Rutledge D y Read G. (1999) Combined coccolith, foraminiferal, and biomarker reconstruction of paleoceanographic conditions over the past $120 \mathrm{kyr}$ in the northern North Atlantic $\left(59^{\circ} \mathrm{N}, 23^{\circ} \mathrm{W}\right)$. Paleoceanography 14: 336-349.

Weaver PPE y Pujol C. (1988) History of the last deglaciation in the Alboran Sea (western Mediterranean) and adjacent north Atlantic as revealed by coccolith floras. Palaeogeography, Palaeoclimatology, Palaeoecology 64: 35-42.

Winter A, Jordan RW y Roth PH. (1994) Biogegraphy of living coccolithophores in ocean waters. En: Winter A y Siesser WG (eds) Coccolithophores. Cambridge: University Press, 161-178.

Winter A, Reiss Z y Luz B. (1979) Distribution of living coccolithophore assemblages in the Gulf of Elat ('Aqaba). Marine Micropaleontology 4: 197-223.

Winter A y Siesser WG. (1994) Coccolithophores. Cambridge Univ. Press. Cambridge, U. K., 242.

Young JR. (1994) Function of coccoliths. En: Winter A y Siesser WG (eds) Coccolithophores. Cambridge: Cambridge University Press, 63-82.

Young JR, Geisen M, Cros L, Kleijne A, Sprengel C, Probert I y Østergaard J. (2003) A guide to extant coccolithophore taxonomy. Journal of Nannoplankton Research Special Issue 1: 1-125.

Young JR y Henriksen K. (2003) Biomineralization Within Vesicles: The Calcite of Coccoliths. Reviews in Mineralogy and Geochemistry 54: 189-215. 
Ziveri P, Baumann K-H, Böckel B, Bollmann J y Young JR. (2004) Biogeography of selected Holocene coccoliths in the Atlantic Ocean. En: Thierstein HR y Young JR (eds) Coccolithophores: from molecular processes to global impact. Springer Berlin Heidelberg, 403-428.

Ziveri P, Thunell RC y Rio D. (1995) Export production of coccolithophores in an upwelling region: Results from San Pedro Basin, Southern California Borderlands. Marine Micropaleontology 24: 335-358.

Zondervan I. (2007) The effects of light, macronutrients, trace metals and CO2 on the production of calcium carbonate and organic carbon in coccolithophores: A review. Deep Sea Research Part II: Topical Studies in Oceanography 54: 521-537. 



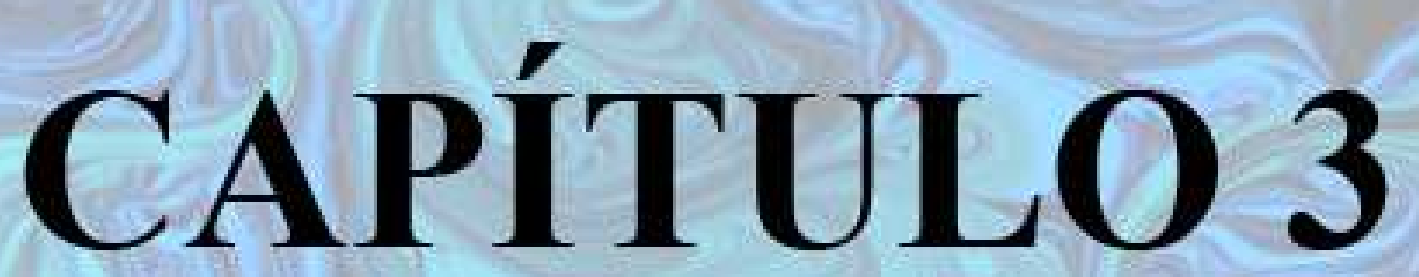





\section{3.-MATERIALES Y TÉCNICAS}

\section{1.-MATERIALES}

\subsection{1.-Testigo CEUTA10PC08}

El testigo de pistón CEUTA10PC08 fue recuperado durante la campaña CONTOURIBER realizada entre el 17 de septiembre y el 14 de octubre de 2010 a bordo del buque oceanográfico BIO Sarmiento de Gamboa (Figura 3.1). El testigo fue recuperado en el sector más occidental del mar de Alborán (Figura 3.2), próximo al estrecho de Gibraltar (latitud 36' $122^{\prime \prime} \mathrm{N}$, longitud $4^{\circ} 52^{\prime} 3^{\prime \prime}$ O) a 914 metros por debajo del nivel mar.

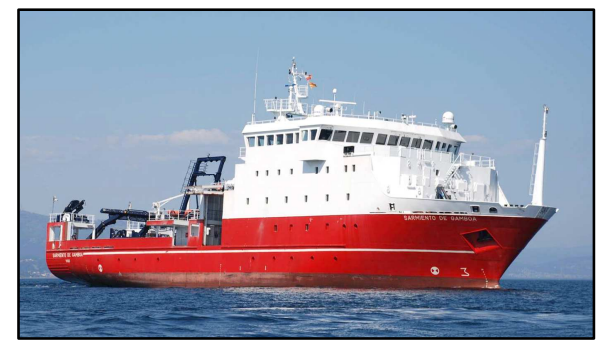

Figura 3.1. Buque oceanográfico BIO Sarmiento de Gamboa del Consejo Superior de Investigaciones Científicas (CSIC). Fuente: http://www.csic.es/buqueoceanografico-garmiento-de-gamboa.

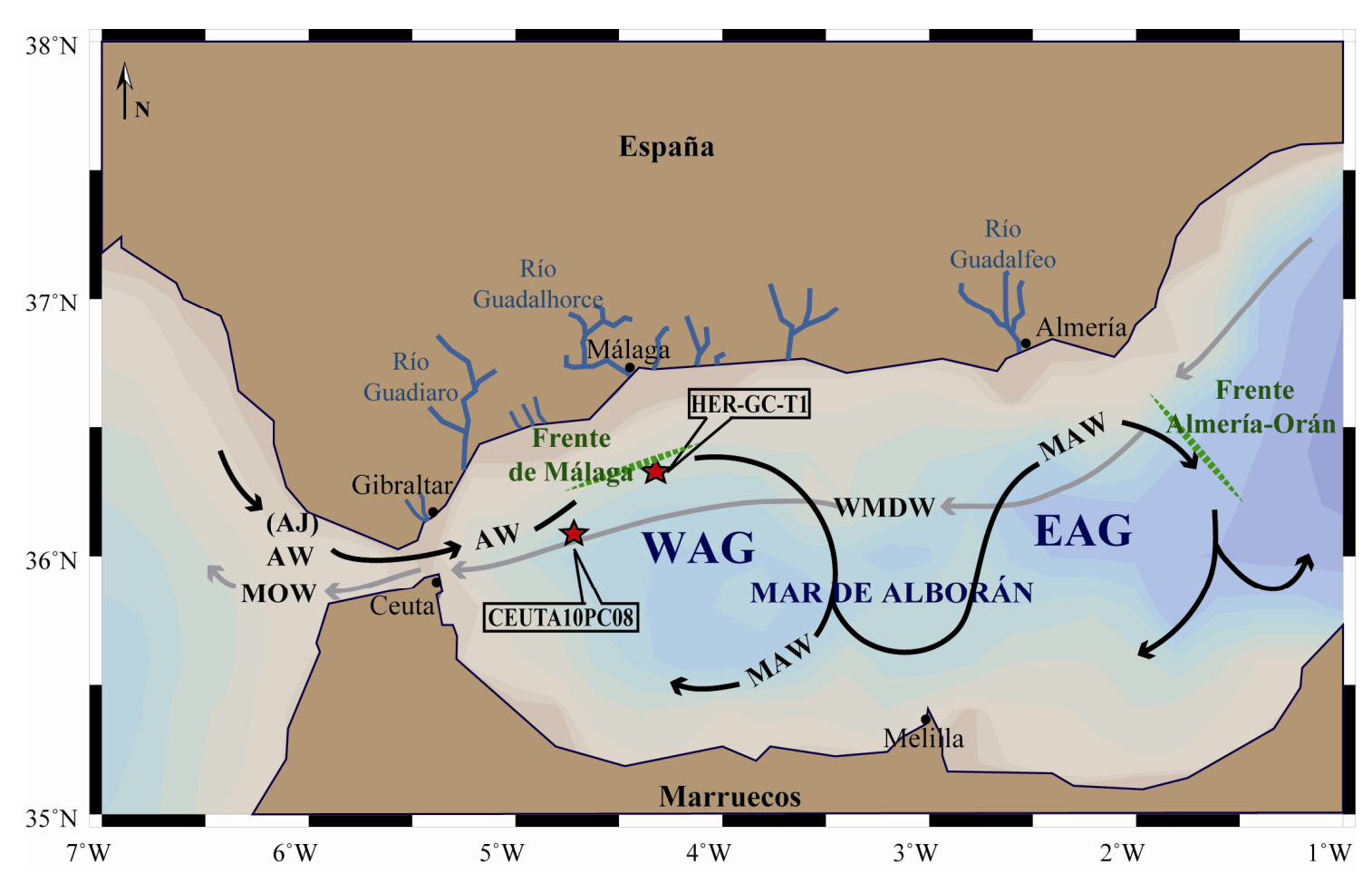

Figura 3.2. Localización de los testigos de sedimento CEUTA10PC08 y HER-GC-T1 en el mar de Alborán, marcados con una estrella roja. Leyenda: AW: Atlantic Water, AJ: Atlantic Jet, MAW: Modified Atlantic Water, WMDW: Western Mediterranean Deep Water, WAG: Western Anticyclonic Gyre, EAG: Eastern Anticyclonic Gyre. 
El sondeo se localiza en la cresta de un "drift" contornítico de tipo elongado separado (Faugères et al., 1999). Este drift se extiende a lo largo de unos $20 \mathrm{~km}$ y cuenta con una anchura variable menor a 4 km, y está lateralmente asociado a un surco erosivo contornítico (Figura 3.3). Este sistema surco-drift se desarrolla al pie del escarpe construido por otro drift contornítico de tipo adosado (el "Drift de Ceuta"; Ercilla et al. (2002)), y que es responsable de la terraza morfológica que caracteriza el talud continental occidental del margen de Marruecos. La formación y desarrollo del drift contornítico donde se localiza el testigo de sedimento resulta del proceso de "retrabajamiento" producido por la corriente de fondo asociada a la WMDW en su salida hacia el estrecho de Gibraltar (Ercilla et al., enviado).

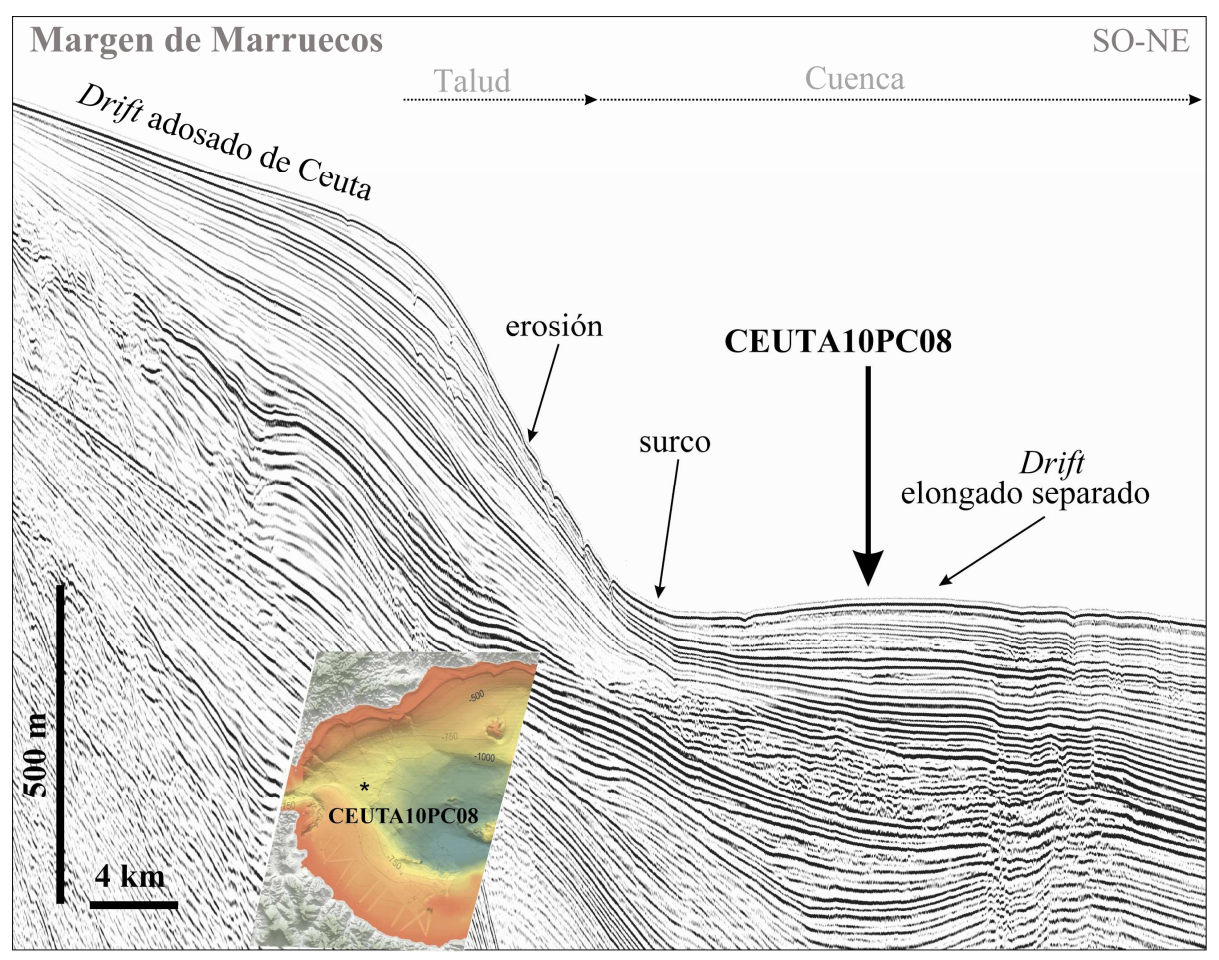

Figura 3.3. Perfil sísmico del sistema surco-drift en el que se localiza el testigo CEUTA10PC08.

El testigo CEUTA10PC08 presenta una longitud de $648 \mathrm{~cm}$ y está compuesto por limo calcáreo arcilloso de color marrón más amarillento hacia techo (Figura 3.4). Presenta abundante bioturbación y dos llamativas capas de materia orgánica entre los centímetros 577 y 581 y los centímetros 598 y 601 (Figura 3.4).

\subsubsection{1-Cronoestratigrafía}

El modelo de edad adoptado para el testigo CEUTA10PC08 está basado en quince edades obtenidas mediante la técnica de espectrometría de masas (AMS) de datación por carbono-14, basadas en muestras monoespecíficas de conchas de foraminíferos pertenecientes a las especies Globigerina inflata, Neogloboquadrina pachyderma (dextral) o Globigerina bulloides (Tabla 3.1). Dichas edades de radiocarbono fueron convertidas posteriormente a edades calendario usando la versión 4.2 del software OxCal (Bronk, 2008) y la curva de calibración Marine13 
(Reimer et al., 2013) que incluye la corrección global de 400 años para el efecto del reservorio marino. La diferencia regional con este reservorio global $(\Delta \mathrm{R})$ (Stuiver y Reimer, 1993) resultó en $-22 \pm 35$ años (Siani et al., 2000) y también fue considerada. El modelo de edad (Figura $3.5 b)$ se realizó mediante interpolación lineal entre las quince edades calendario, cubriendo el periodo comprendido entre 25,5 y 4,5 ka y resultando en una tasa de sedimentación media de $37,4 \mathrm{~cm} * \mathrm{ka}^{-1}$ y una resolución temporal media de 70 años.

\section{CEUTA10PC08}

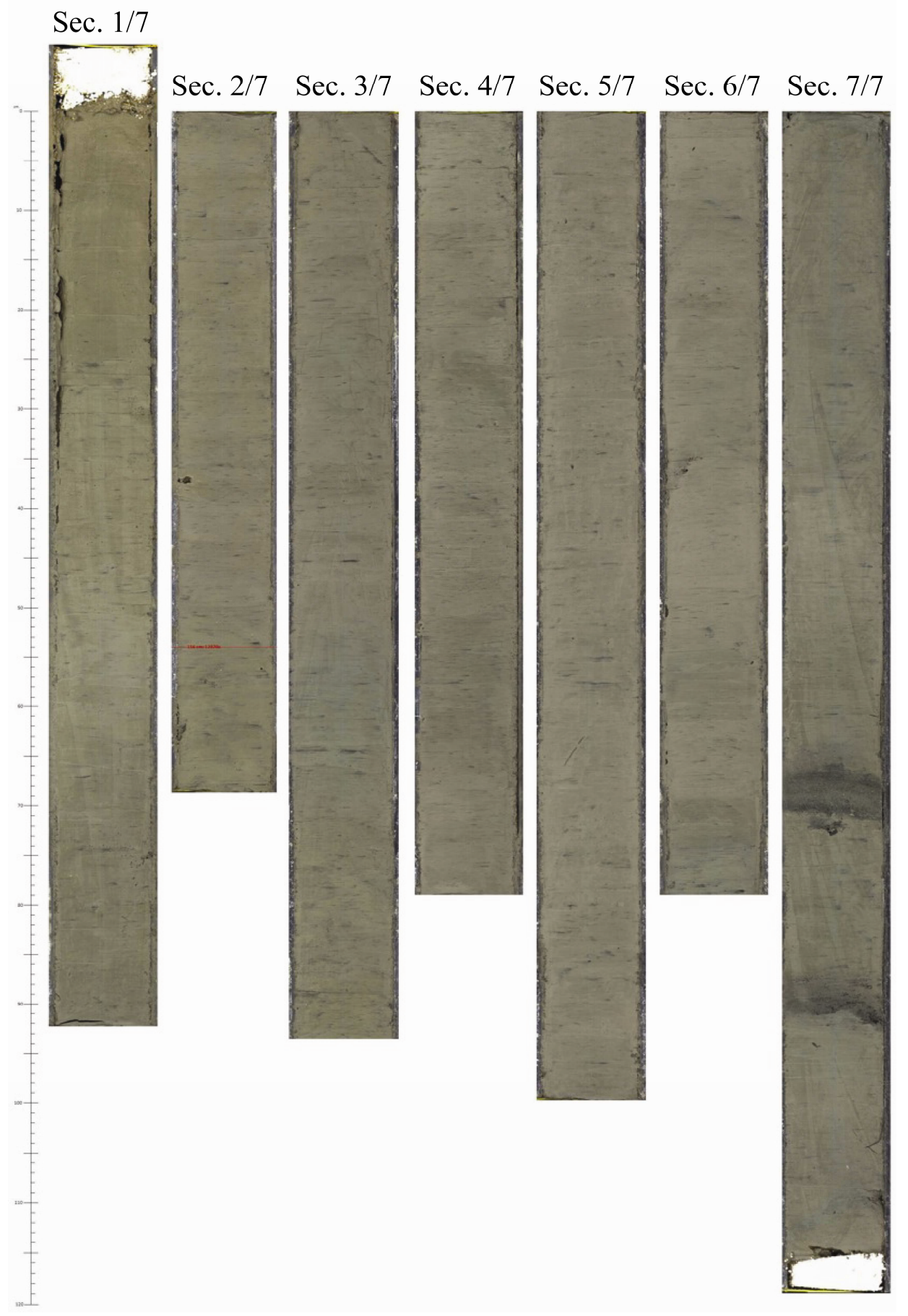

Figura 3.4. Imagen de las siete secciones que componen el testigo de sedimento CEUTA10PC08. 


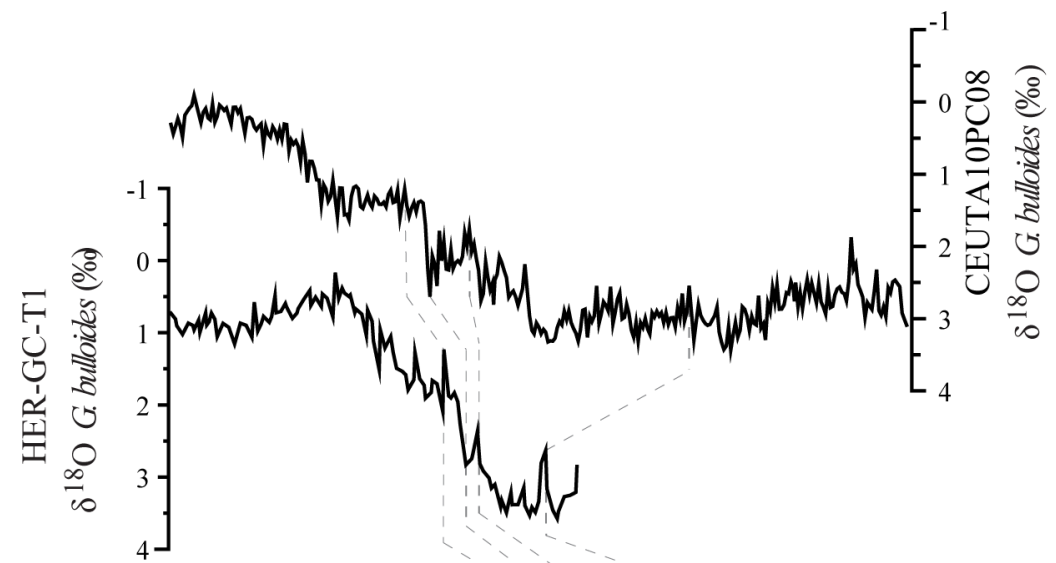

a)
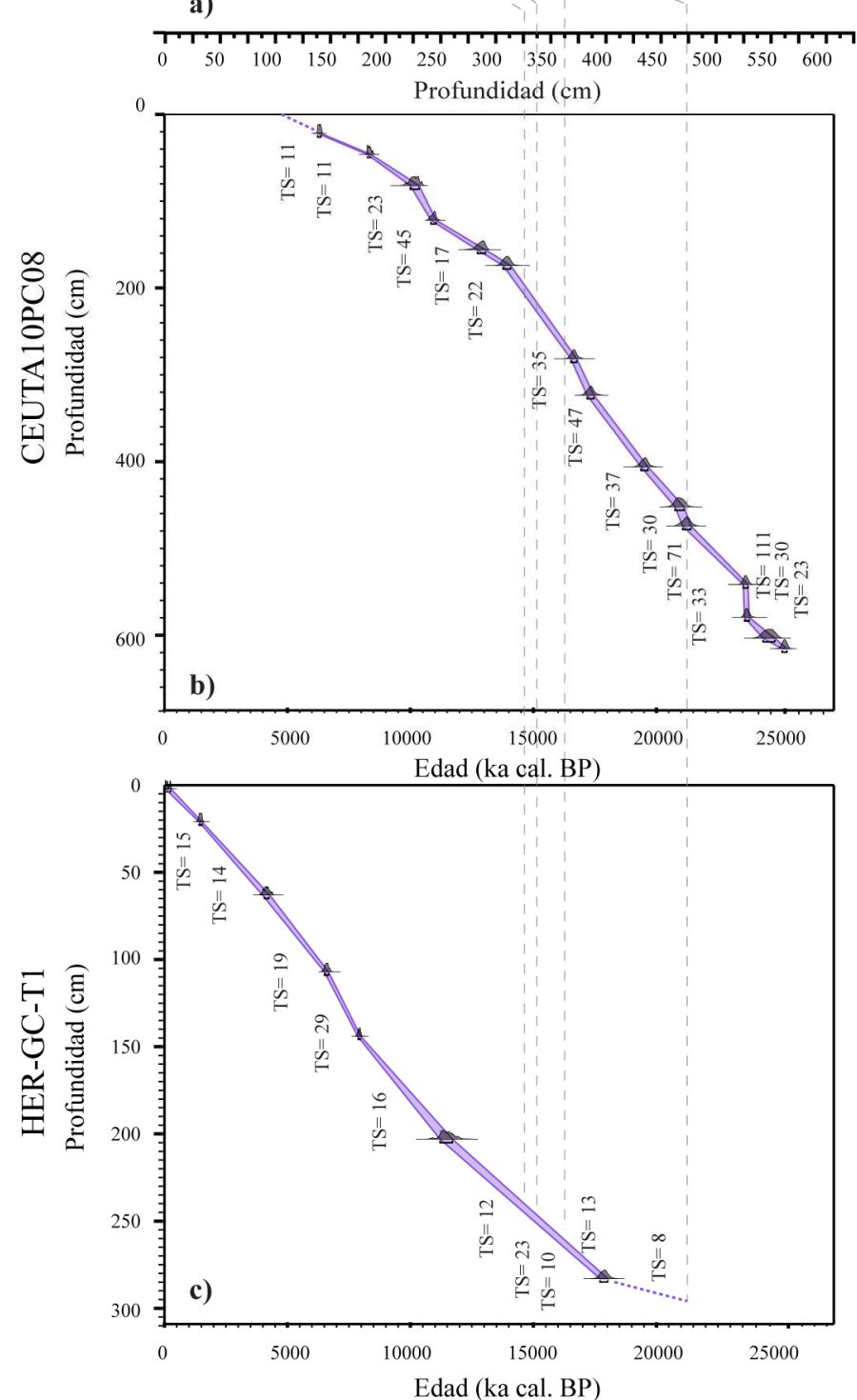

Figura 3.5. Modelos de edad para los testigos CEUTA10PC08 y HER-GC-T1 realizados con el software OxCal versión 4.2 (Bronk, 2008). Las líneas sólidas grises delimitan la probabilidad del 68,2 \%. a) Perfiles de isótopos estables de oxígeno de los testigos CEUTA10PC08 y HER-GC-T1. Las líneas discontinuas unen los puntos obtenidos por correlación (tie points) entre ambos perfiles. Modelo de edad del testigo b) CEUTA10PC08 y c) HER-GC-T1. TS hace referencia a la tasa de sedimentación, expresada en $\mathrm{cm}^{*} \mathrm{ka}^{-1}$. 
Tabla 3.1. Dataciones realizadas para la construcción del modelo de edad del testigo de sedimento CEUTA10PC08.

\begin{tabular}{|c|c|c|c|c|}
\hline Código muestra & Especie & $\begin{array}{c}\text { Profundidad } \\
\text { (cm) }\end{array}$ & $\begin{array}{c}\text { Edad } \\
\text { (a) }\end{array}$ & $\begin{array}{c}\text { Edad } \\
\text { calendario } \\
\text { (a cal BP) }\end{array}$ \\
\hline $\begin{array}{c}\text { CEUTA10PC08_22 } \\
/ \\
\text { Poz-56516 }\end{array}$ & G. inflata & 22 & $5870 \pm 40$ & $6312 \pm 56$ \\
\hline $\begin{array}{c}\text { CEUTA10PC08_46 } \\
/ \\
\text { Poz-56517 }\end{array}$ & N. pachyderma (dex) & 46 & $7940 \pm 40$ & $8426 \pm 56$ \\
\hline $\begin{array}{c}\text { CEUTA10PC08_82 } \\
/ \\
\text { Poz-56518 }\end{array}$ & N. pachyderma (dex) & 82 & $9190 \pm 50$ & $10006 \pm 100$ \\
\hline $9977^{\mathrm{b}}$ & N. pachyderma (dex) & 122 & $9900 \pm 30$ & $10885 \pm 91$ \\
\hline $9552^{b}$ & G. bulloides & 156 & $11410 \pm 30$ & $12890 \pm 79$ \\
\hline $10602^{b}$ & N. pachyderma (dex) & 174 & $12240 \pm 70$ & $13717 \pm 107$ \\
\hline $9979^{\mathrm{b}}$ & N. pachyderma (dex) & 281,5 & $14270 \pm 40$ & $16818 \pm 125$ \\
\hline $9980^{b}$ & N. pachyderma (dex) & 323,5 & $14920 \pm 40$ & $17720 \pm 97$ \\
\hline $9981^{b}$ & $N$. pachyderma (dex) & 406 & $16910 \pm 40$ & $19936 \pm 104$ \\
\hline $9982^{b}$ & N. pachyderma (dex) & 452 & $18110 \pm 50$ & $21462 \pm 128$ \\
\hline $9983^{b}$ & N. pachyderma (dex) & 474 & $18360 \pm 50$ & $21773 \pm 106$ \\
\hline $9984^{b}$ & N. pachyderma (dex) & 541,5 & $20170 \pm 50$ & $23820 \pm 115$ \\
\hline $10603^{b}$ & G. bulloides & 579,5 & $20480 \pm 60$ & $24162 \pm 113$ \\
\hline $10604^{b}$ & G. bulloides & 603,5 & $21100 \pm 60$ & $24967 \pm 173$ \\
\hline $10605^{b}$ & N. pachyderma (dex) & 615,5 & $21540 \pm 60$ & $25500 \pm 111$ \\
\hline
\end{tabular}

\footnotetext{
${ }^{a}$ Hace referencia a las edades de radiocarbono medidas en el Poznan Radiocarbon Laboratory.

${ }^{\mathrm{b}}$ Hace referencia a las edades de radiocarbono medidas en el Center for Applied Isotope Studies of University of Georgia.
}

\subsection{2.-Testigo HER-GC-T1}

El testigo HER-GC-T1 fue recuperado durante la campaña HERMESIONE realizada entre el 15 de septiembre y el 9 de octubre de 2009 en el mar de Alborán a bordo del buque de investigación oceanográfica BIO Hespérides de la armada española (Figura 3.6).

La campaña HERMESIONE se realizó con diversos objetivos en función de las zonas geográficas en las que se subdividió la cuenca. Para la región próxima al margen de Málaga a la 
que pertenece el testigo HER-GC-T1, el objetivo fue obtener varios testigos de gravedad y multitestigos para la realización de análisis paleoceanográficos.

El testigo de gravedad HER-GC-T1 fue recuperado próximo al margen de Málaga $\left(36^{\circ} 22^{\prime} 12^{\prime \prime} \mathrm{N}, 4^{\circ} 17^{\prime} 57^{\prime \prime} \mathrm{O}\right)$ a 658,9 metros por debajo del nivel del mar (Figura 3.3) y presenta una longitud de $374 \mathrm{~cm}$ (Figura 3.7). Está formado por limos calcáreos y presenta tonos amarillentos a techo y una transición hacia muro de tonos marrón claro, gris oliva y gris oscuro (Figura 3.7). Muestra varios signos de bioturbación y pequeños fragmentos de conchas, sobre todo hacia los niveles inferiores donde también se observan finas capas de materia orgánica.

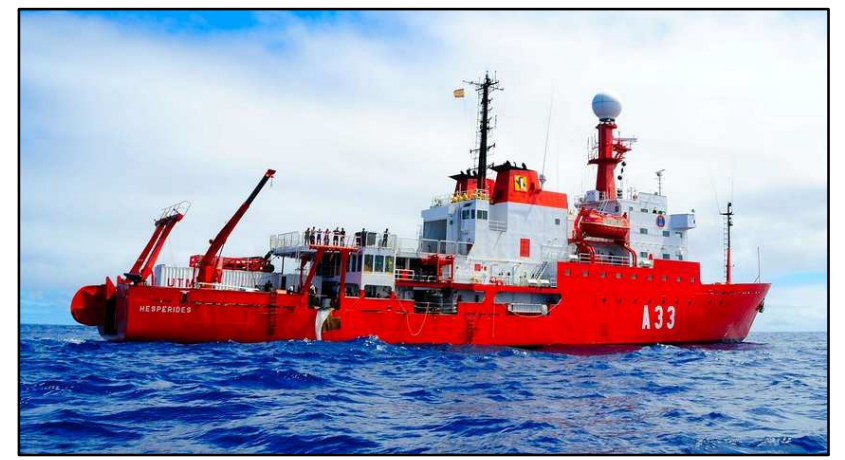

Figura 3.5. Buque oceanográfico BIO Hespérides de la armada española Fuente: http://www.armada.mde.es/ArmadaPortal/page/Portal/Armadaespannola/buques_superficie/prefLang_es/ 12 buques-investigacion oceanografia.

\subsubsection{1.-Cronoestratigrafia}

Para la realización del modelo de edad del testigo HER-GC-T1 se determinaron siete edades de radiocarbono (Tabla 3.2) mediante la técnica AMS a partir de conchas de foraminíferos de la especie Globigerina inflata y/o Neogloboquadrina pachyderma (dextral). Como en el caso del testigo CEUTA10PC08, estas edades fueron convertidas posteriormente a edades calendario usando el software OxCal versión 4.2 (Bronk, 2008) y la curva de calibración Marine13 (Reimer et al., 2013). La corrección global de 400 años para el efecto del reservorio marino y la diferencia regional $(\Delta \mathrm{R})$ de $-22 \pm 35$ años (Siani et al., 2000) también fueron consideradas.

Además de estas siete edades calendario, se consideraron tres puntos de correlación (tie points) adicionales obtenidos a partir de la correlación entre los perfiles isotópicos de oxígeno de los testigos HER-GC-T1 y CEUTA10PC08 (Figura 3.5a), debido a que este último testigo posee un modelo de edad más preciso. 


\section{HER-GC-T1}

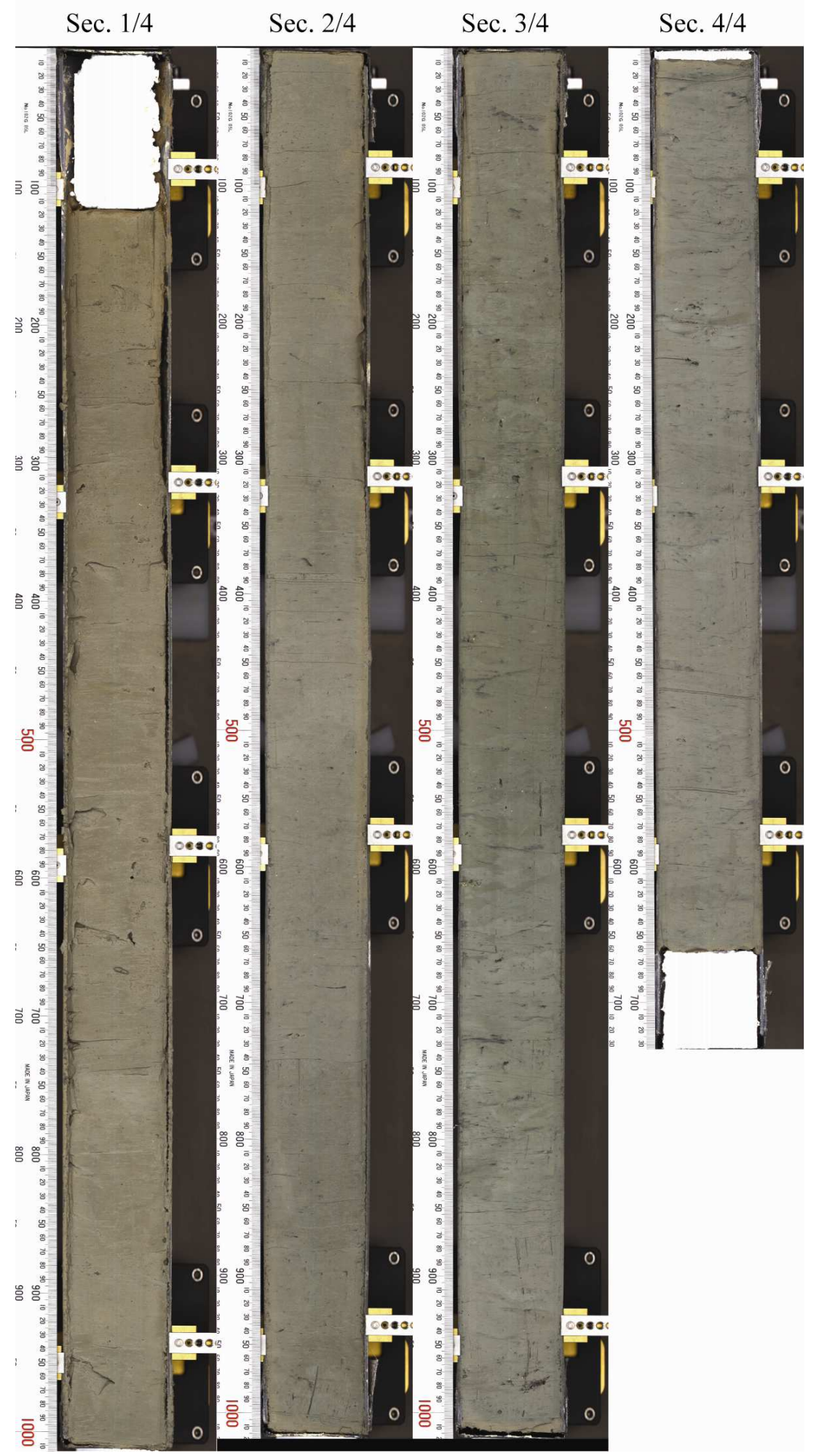

Figura 3.7. Imagen de las cuatro secciones que componen el testigo de sedimento HER-GC-T1.

El modelo de edad (Figura 3.5c) se realizó mediante interpolación lineal entre los 10 puntos de control, cubriendo el periodo desde 23,5 hasta 0,09 ka con una tasa de sedimentación media de $16,6 \mathrm{~cm}^{*} \mathrm{ka}^{-1} \mathrm{y}$ una resolución temporal media de 140 años. 
Tabla 3.2. Dataciones realizadas para la construcción del modelo de edad del testigo de sedimento HERGC-T1

\begin{tabular}{|c|c|c|c|c|}
\hline Código muestra & Especie & $\begin{array}{c}\text { Profundidad } \\
\text { (cm) }\end{array}$ & $\begin{array}{c}\text { Edad } \\
\text { (a) }\end{array}$ & $\begin{array}{c}\text { Edad } \\
\text { calendario } \\
\text { (a cal BP) }\end{array}$ \\
\hline SEC1_2/ Poz-53233 ${ }^{\mathrm{a}}$ & G. inflata & 2 & $440 \pm 25$ & $88 \pm 62$ \\
\hline SEC1_21/OS-87586 ${ }^{\mathrm{b}}$ & G. inflata & 21 & $1810 \pm 25$ & $1379 \pm 54$ \\
\hline SEC1_63/Poz-53234 ${ }^{\mathrm{a}}$ & G. inflata & 63 & $4175 \pm 35$ & $4284 \pm 76$ \\
\hline SEC2_17/ Poz-53235 ${ }^{\mathrm{a}}$ & G. inflata & 107 & $6100 \pm 40$ & $6550 \pm 68$ \\
\hline SEC2_54/OS-87587 & $\begin{array}{c}\text { G. inflata }+N . \\
\text { pachyderma }(\mathrm{dex})\end{array}$ & 144 & $7350 \pm 35$ & $7834 \pm 58$ \\
\hline SEC3_12/ Poz-53236 ${ }^{\mathrm{a}}$ & N. pachyderma $(\mathrm{dex})$ & 202 & $10400 \pm 60$ & $11539 \pm 162$ \\
\hline SEC3_48 & & 238 & & 14582 \\
\hline $\mathrm{SEC} 3{ }_{-} 67^{\mathrm{c}}$ & & 257 & & 15217 \\
\hline SEC $3 \_77^{\mathrm{c}}$ & & 267 & & 16198 \\
\hline SEC3_92/Poz-53237 & N. pachyderma (dex) & 282 & $15180 \pm 80$ & $18006 \pm 121$ \\
\hline SEC4_36 $6^{\mathrm{c}}$ & & 326 & & 21396 \\
\hline
\end{tabular}

${ }^{a}$ Hace referencia a las edades de radiocarbono medidas en el Poznan Radiocarbon Laboratory.

${ }^{\mathrm{b}}$ Hace referencia a las edades de radiocarbono medidas en el Center for Applied Isotope Studies of University of Georgia.

${ }^{\mathrm{c}}$ Hace referencia a los tie points: puntos obtenidos por correlación entre los perfiles isotópicos de los testigos CEUTA10PC08 y HER-GC-T1.

\subsection{3.-Base de datos actual}

Con el fin de desarrollar una función de transferencia se utilizó una base de datos de muestras actuales. Ésta consiste en un conjunto de muestras de sedimento superficial (base de datos biológica) y datos de varias variables ambientales (base de datos ambiental) correspondientes a las localizaciones de cada una de las muestras de sedimento superficial.

\subsubsection{1.-Base de datos biológica: muestras de sedimento superficial}

Con el fin de crear una base de datos actual se muestrearon los core-top de 118 testigos de sedimento almacenados en la Universidad de Vigo y el repositorio de muestras del fondo marino del Instituto de Ciencias Marinas (CSIC, Barcelona) (Figura 3.8, Tabla 3.3). 


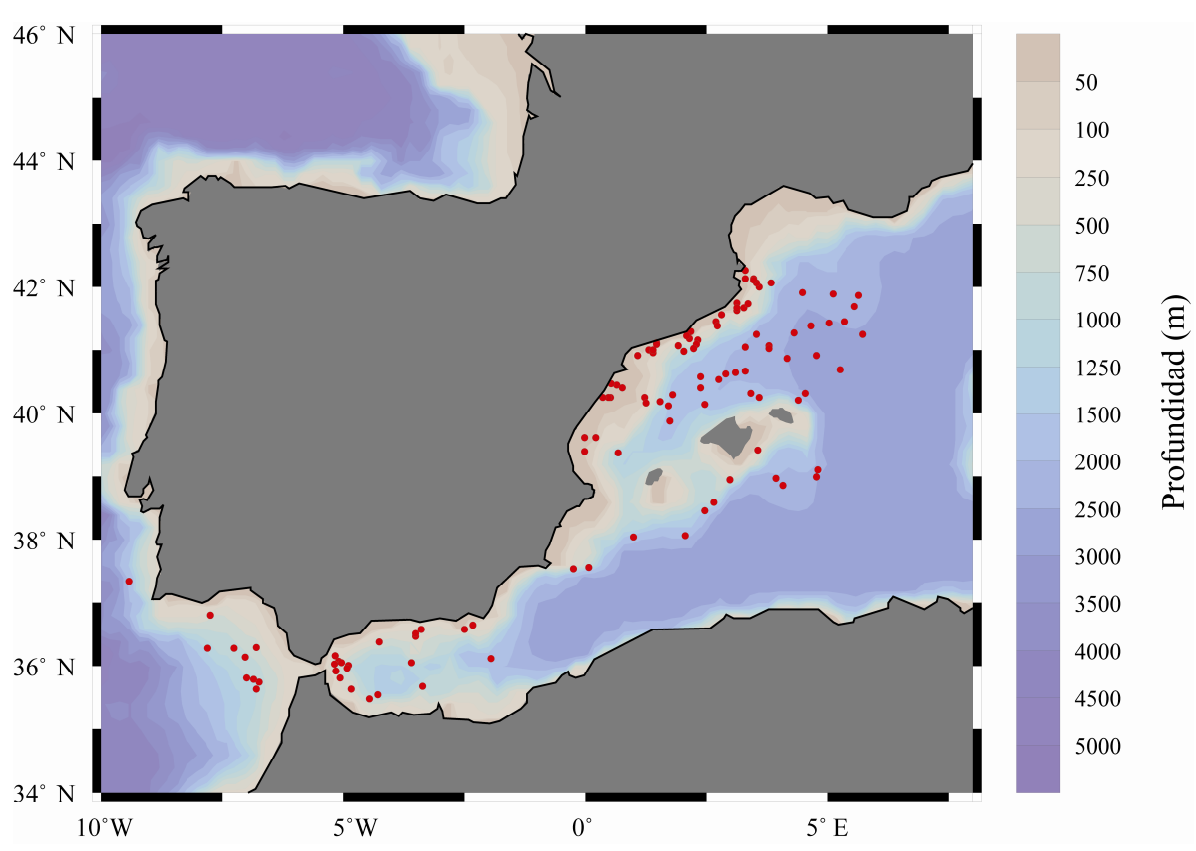

Figura 3.8. Localización de las 118 muestras de sedimento que constituyen la base de datos actual inicial en el mar Mediterráneo occidental y el océano Atlántico, marcadas con puntos rojos.

Este último cuenta con una herramienta de búsqueda en línea (http://ww2.icm.csic.es/geo/gma/SurveyMaps/) que permite visualizar la localización de todos los sondeos disponibles en dicho instituto, la campaña en la que fueron recuperados, su composición y otros datos de utilidad.

Tabla 3.3. Muestras de sedimento superficial y sus coordenadas consideradas en la base de datos actual inicial. a) Muestras almacenadas en el repositorio de muestras del fondo marino del Instituto de Ciencias Marinas (CSIC, Barcelona). b) Muestras almacenadas en la Universidad de Vigo.

\begin{tabular}{|l|l|l|l|l|l|l|l|}
\hline Muestra & Procedencia & Latitud & Longitud & Muestra & Procedencia & Latitud & Longitud \\
\hline STR-93/TG1 & a & 36,052 & $-3,585$ & GC-88-3/TG-15 & a & 36,164 & $-7,000$ \\
\hline VALSIS/KF-16 & a & 41,249 & 3,532 & GC-83-2/TR-440 & a & 40,972 & 1,406 \\
\hline CO-81-2/TK-2 & a & 36,133 & $-1,926$ & CO-81-1/K-61 & a & 41,693 & 5,567 \\
\hline MAYC96/MTL-1 & a & 36,467 & $-3,502$ & CO-83-2/K-17 & a & 41,033 & 3,788 \\
\hline $\begin{array}{l}\text { CO-80-4/K-25 } \\
\text { (sec 1) (A) }\end{array}$ & a & 38,597 & 2,677 & VALSIS/KF-2 & a & 39,372 & 0,692 \\
\hline CG-90-1/TG-5 & a & 36,385 & $-4,250$ & VALSIS/KF-13 & a & 40,682 & 3,304 \\
\hline STR93/TG-11 & a & 36,069 & $-5,067$ & CO-83-2/K-8 & a & 40,662 & 3,113 \\
\hline CO-81-1/K-87 & a & 41,058 & 3,307 & GC-88-1/TG-44 & a & 42,064 & 3,527 \\
\hline GC-82-1/TR-137 & a & 41,007 & 1,337 & CO-83-2/K-49 & a & 40,712 & 5,278 \\
\hline CO-83-1/K-29 & a & 40,252 & 1,260 & VALSIS/KF-5 & a & 40,157 & 2,483 \\
\hline CO-83-1/K-10 & a & 40,183 & 1,288 & CO-81-17/K-60 & a & 41,878 & 5,657 \\
\hline CO-81-1/TK-10 & a & 40,309 & 1,807 & GC-93-1/TG3 & a & 41,166 & 2,331 \\
\hline GC-93-1/TC-3 & a & 41,303 & 2,176 & CO-80-47K-34 & a & 40,218 & 4,410 \\
\hline GC-84-6/TR-552 & a & 41,638 & 3,138 & $\begin{array}{l}\text { CO-80-4/K-10 } \\
\text { (sec1)(A) }\end{array}$ & a & 38,050 & 1,015 \\
\hline GC-84-6/TR-598 & a & 41,753 & 3,143 & CO-81-1/K-56 & a & 41,882 & 5,126 \\
\hline GC-84-6/TR-606 & a & 41,677 & 3,294 & GC-82-1/TR-76 & a & 41,109 & 2,294 \\
\hline
\end{tabular}




\begin{tabular}{|c|c|c|c|c|c|c|c|}
\hline MAYC96/CD-2 & $\mathrm{a}$ & 36,036 & $-5,170$ & GC-82-1/TR-281 & $\mathrm{a}$ & 41,012 & 1,402 \\
\hline VALSIS/KF-7 & $\mathrm{a}$ & 40,405 & 2,382 & E-3D-78/35388 & $\mathrm{a}$ & 39,000 & 4,783 \\
\hline $\begin{array}{l}\text { CO-80-4/K-16 } \\
(\sec 1)(\mathrm{A})\end{array}$ & $\mathrm{a}$ & 38,071 & 2,072 & CO-80-4/K-38 & $\mathrm{a}$ & 40,258 & 3,600 \\
\hline $\mathrm{CO}-80-4 / \mathrm{K}-37$ & $\mathrm{a}$ & 40,334 & 4,541 & GC-84-6/TR-565 & $\mathrm{a}$ & 41,680 & 3,149 \\
\hline CO-80-4 /K-39 & $\mathrm{a}$ & 40,331 & 3,442 & GC-88-1/TR-270 & $\mathrm{a}$ & 40,837 & 1,205 \\
\hline 80-CL/TK-33 & $\mathrm{a}$ & 39,633 & $-0,001$ & GC-84-6/TR-442 & $\mathrm{a}$ & 41,452 & 2,724 \\
\hline 80-CL/TK-30 & $\mathrm{a}$ & 39,633 & 0,217 & GC-82-1/TR-294 & $\mathrm{a}$ & 41,127 & 1,487 \\
\hline $\mathrm{CO}-80-4 / \mathrm{K}-4$ & $\mathrm{a}$ & 37,540 & $-0,243$ & VALSIS/KF-17 & $\mathrm{a}$ & 41,276 & 4,335 \\
\hline $\mathrm{CO}-80-3 / \mathrm{K}-2$ & $\mathrm{a}$ & 39,417 & $-0,020$ & GC-85-6/TG-10 & $\mathrm{a}$ & 41,379 & 2,752 \\
\hline $\mathrm{CO}-80-4 / \mathrm{K}-33$ & $\mathrm{a}$ & 39,115 & 4,835 & GC-93-1/TC-38 & $\mathrm{a}$ & 40,989 & 2,059 \\
\hline MAYC-96/MTL-2 & $\mathrm{a}$ & 36,526 & $-3,474$ & GC-82-1/TR-299 & $\mathrm{a}$ & 41,096 & 1,486 \\
\hline MAYC-96/MTL-18 & $\mathrm{a}$ & 36,582 & $-3,376$ & $\mathrm{CO}-83-2 / \mathrm{K}-7$ & $\mathrm{a}$ & 40,620 & 2,933 \\
\hline MAYC-96/ALM-22 & $\mathrm{a}$ & 36,664 & $-2,333$ & VALSIS/KF-12 & $\mathrm{a}$ & 41,033 & 2,241 \\
\hline MAYC-96/ALM-19 & $\mathrm{a}$ & 36,589 & $-2,481$ & CO-80-4/K-9 & $\mathrm{a}$ & 37,570 & 0,092 \\
\hline MAYC-96/GDR-4 & $\mathrm{a}$ & 36,182 & $-5,142$ & $\mathrm{CO}-83-2 / \mathrm{K}-6$ & $\mathrm{a}$ & 40,547 & 2,777 \\
\hline GC-92-1/TG-6 & $\mathrm{a}$ & 40,458 & 0,662 & CO-81-1/K-16 & $\mathrm{a}$ & 40,141 & 1,741 \\
\hline GC-92-1/TG-1 & $\mathrm{a}$ & 40,491 & 0,566 & GC-88-1/TR-144 & $\mathrm{a}$ & 42,129 & 3,492 \\
\hline GC-93-1/TC-35 & $\mathrm{a}$ & 41,073 & 1,915 & CO-81-1/K-74 & $\mathrm{a}$ & 40,880 & 4,183 \\
\hline GC-88-1/TG-12 & $\mathrm{a}$ & 42,155 & 3,308 & GC-84-6/TR-477 & $\mathrm{a}$ & 41,573 & 2,831 \\
\hline GC-88-1/TG-15 & $\mathrm{a}$ & 42,137 & 3,499 & GC-82-1/TR-235 & $\mathrm{a}$ & 40,921 & 1,099 \\
\hline VALSIS-1/KF-4 & $\mathrm{a}$ & 39,879 & 1,754 & CO-83-2/K-43 & $\mathrm{a}$ & 40,925 & 4,770 \\
\hline STR-93/TG-8 & $\mathrm{a}$ & 35,818 & $-5,052$ & VALSIS/KF-18 & $\mathrm{a}$ & 41,406 & 4,674 \\
\hline E-3D-78/35418 & $\mathrm{a}$ & 38,950 & 3,008 & GC-88-1/TR-72 & $\mathrm{a}$ & 42,272 & 3,304 \\
\hline GC-88-1/TR-70 & $\mathrm{a}$ & 42,297 & 3,318 & GC-84-1/TR-111 & $\mathrm{a}$ & 41,726 & 3,370 \\
\hline $79 \mathrm{KEB} / \mathrm{K}-30$ & $\mathrm{a}$ & 40,410 & 0,793 & GC-82-1/TR-17 & $\mathrm{a}$ & 41,236 & 2,087 \\
\hline 80-CL/TK-61 & $\mathrm{a}$ & 40,252 & 0,533 & GC-88-1/TG-38 & $\mathrm{a}$ & 42,059 & 3,843 \\
\hline CL-83-1/K-104 & $\mathrm{a}$ & 41,185 & 2,151 & VALSIS/KF-9 & $\mathrm{a}$ & 40,580 & 2,394 \\
\hline E-30-78/35411 (pilot) & $\mathrm{a}$ & 39,420 & 3,553 & CEUTA/MC/1 & $\mathrm{b}$ & 35,934 & $-5,120$ \\
\hline STR93/TG-13 & $\mathrm{a}$ & 36,068 & $-5,017$ & CEUTA/MC/4 & $\mathrm{b}$ & 35,648 & $-4,818$ \\
\hline CO-80-4/K-30 & $\mathrm{a}$ & 38,970 & 3,949 & CEUTA/MC/8 & $\mathrm{b}$ & 36,023 & $-4,867$ \\
\hline 80-CL/TK-60 & $\mathrm{a}$ & 40,251 & 0,469 & CEUTA/MC/7 & $\mathrm{b}$ & 35,971 & $-4,912$ \\
\hline 80-CL/TK-59 & $\mathrm{a}$ & 40,250 & 0,384 & CEUTA/BC/5 & $\mathrm{b}$ & 35,624 & $-5,145$ \\
\hline CO-83-2/K-16 & $\mathrm{a}$ & 41,073 & 3,790 & CEUTA/MC/2 & $\mathrm{b}$ & 35,493 & $-4,448$ \\
\hline CO-81-1/K-64 & $\mathrm{a}$ & 41,257 & 5,725 & CÁDIZ10/BC/4 & $\mathrm{b}$ & 35,737 & $-6,732$ \\
\hline GC-88-1/T-G30 & $\mathrm{a}$ & 42,013 & 3,612 & CÁDIZ10/MC/8 & $\mathrm{b}$ & 35,821 & $-6,968$ \\
\hline CO-81-2/TR-62 & $\mathrm{a}$ & 35,552 & $-4,283$ & CÁDIZ10/BC/2 & $\mathrm{b}$ & 35,798 & $-6,853$ \\
\hline CO-80-4/K-28 & $\mathrm{a}$ & 38,877 & 4,109 & CÁDIZ10/MC/6 & $\mathrm{b}$ & 36,305 & $-6,764$ \\
\hline CO-81-1/K-66 & $\mathrm{a}$ & 41,459 & 5,372 & CÁDIZ10/MC/1 & $\mathrm{b}$ & 35,651 & $-6,784$ \\
\hline $\mathrm{CO}-81-1 / \mathrm{K}-4$ & $\mathrm{a}$ & 40,192 & 1,549 & IODP/GC/5B & $\mathrm{b}$ & 36,286 & $-7,235$ \\
\hline CO-81-1/K-69 & $\mathrm{a}$ & 41,445 & 5,047 & IODP/GC/2A & $\mathrm{b}$ & 36,286 & $-7,808$ \\
\hline STR93/TG-21 & $\mathrm{a}$ & 35,684 & $-3,351$ & IODP/WI/1B & $\mathrm{b}$ & 37,343 & $-9,422$ \\
\hline $\mathrm{CO}-80-4 / \mathrm{K}-23(\mathrm{~W})$ & $\mathrm{a}$ & 38,464 & 2,491 & APL-SHACK4 & $\mathrm{b}$ & 35,572 & $-10,126$ \\
\hline CO-83-2/K-28 & $\mathrm{a}$ & 41,900 & 4,500 & GC/09A/MC & $\mathrm{b}$ & 36,805 & $-7,719$ \\
\hline
\end{tabular}




\subsubsection{2.-Base de datos ambiental: datos de las variables ambientales}

La base de datos ambiental actual se construyó a partir de datos disponibles en el World Ocean Atlas 2013 [WOA13] usando el software Ocean Data View [ODV] (Schlitzer, 2014). Se seleccionaron los datos de temperatura (Locarnini et al., 2013), salinidad (Zweng et al., 2013), clorofila $a$ (Boyer et al., 2013), contenido en oxígeno, saturación de oxígeno (García et al., 2014a) y nutrientes (nitrato, fosfato y silicato) (García et al., 2014b) de la columna de agua de las localizaciones de las muestras de sedimento superficial. Los datos de estas variables, promediados anual y estacionalmente (verano e invierno) desde 1955 hasta 2012, fueron obtenidos para las siguientes profundidades: 10, 20, 30, 50, 75, 100, 125, 150, 200 у 300 metros.

\section{2.-MÉTODOS Y TÉCNICAS}

\subsection{1.-Análisis micropaleontológico para el estudio de las asociaciones cocolitoforales}

\subsubsection{1.-Muestreo}

Para el análisis micropaleontológico de las asociaciones cocolitoforales se muestreó una pequeña cantidad de sedimento húmedo en los testigos CEUTA10PC08 y HER-GC-T1 y los core-tops que conforman la base de datos biológica como se detalla a continuación:

-El testigo HER-GC-T1 fue muestreado cada 2-3 cm obteniendo 143 muestras.

-El testigo CEUTA10PC08 fue muestreado cada $2 \mathrm{~cm}$ obteniendo 297 muestras.

-En los 118 core-tops se muestreó el primer centímetro, o el segundo si el primero no estaba disponible.

Todos los muestreos se realizaron con una espátula de metal y cada muestra fue guardada en una bolsa de plástico etiquetada con su nombre. Seguidamente las muestras se guardaron en el congelador para su posterior liofilización (Anexo II).

\subsubsection{2.-Preparación de muestras}

Para el estudio de las asociaciones cocolitoforales del material de esta tesis doctoral se adoptó la técnica de preparación de muestras de Flores y Sierro (1997). Ésta es una técnica de decantación que permite la obtención de datos cuantitativos como el número de cocolitos por gramo de sedimento o sus flujos. Los pasos generales que contempla esta técnica (Figura 3.9) son:
A) Pesada del sedimento
B) Disolución en agua tamponada
C) Extracción
D) Homogeneización 
E) Extracción de la fase líquida y secado

F) Montaje

Los procedimientos detallados adoptados para la preparación de las muestras pueden consultarse en el Anexo II de esta tesis doctoral.

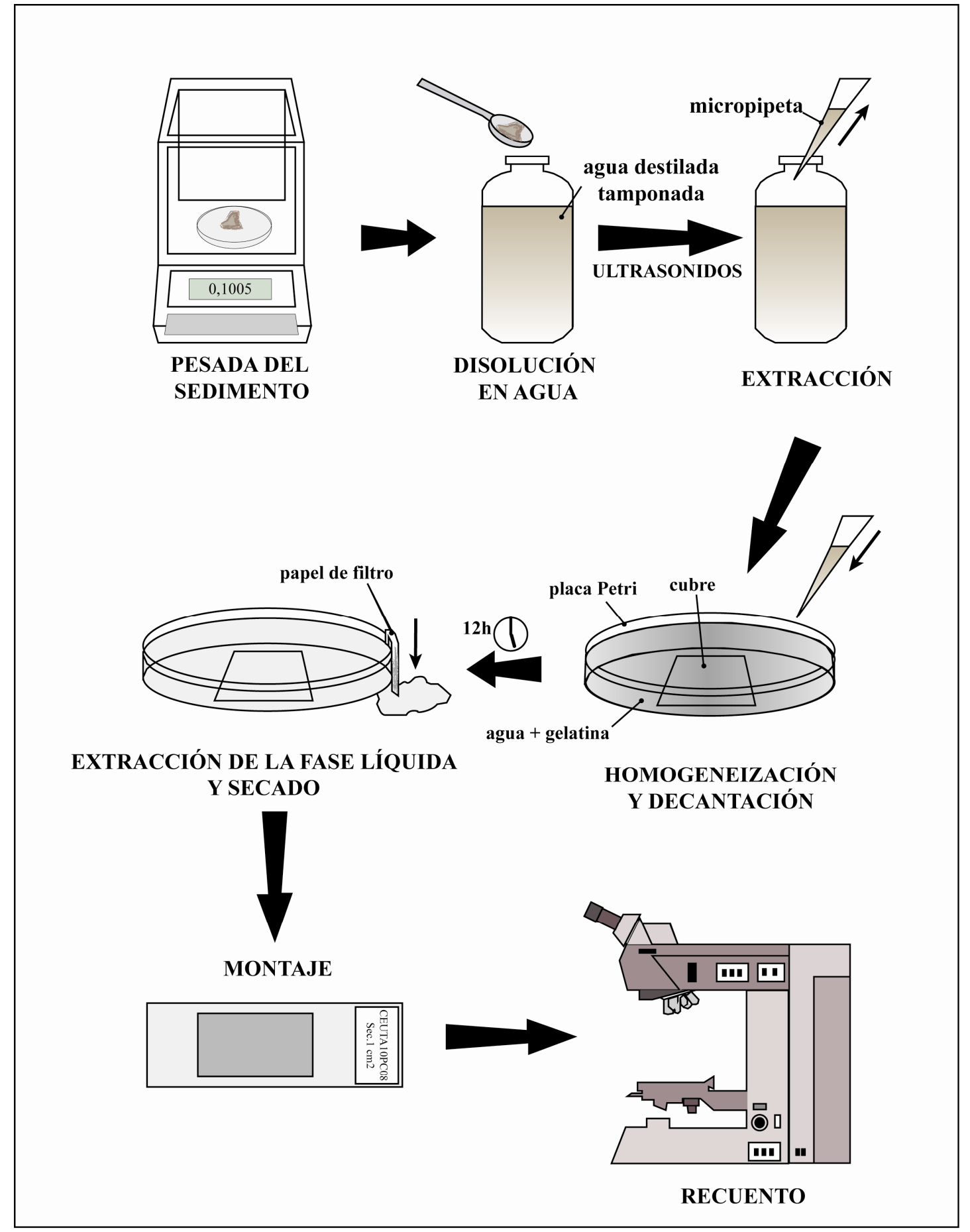

Figura 3.9. Esquema de los pasos contemplados en la técnica de decantación de Flores y Sierro (1997) para la preparación de muestras para su análisis micropaleontológico. 
Es posible que la concentración de los cocolitóforos en las muestras preparadas para su observación al microscopio sea mucho mayor o menor que la esperada, dificultando su identificación en el primer caso o ralentizando el conteo en el segundo. Por este motivo se seleccionaron unas pocas muestras al azar y se prepararon a diferentes concentraciones, combinando 0,1 o $0,2 \mathrm{~g}$ de sedimento con 100 o $200 \mu \mathrm{L}$ de volumen pipeteado, siguiendo la técnica de decantación de Flores y Sierro (1997) que se detalla en el Anexo II. Tras su observación al microscopio se determinó que la concentración más adecuada a la que se debían preparar el resto de muestras fue $0,1 \mathrm{~g}$ de sedimento y $100 \mu \mathrm{L}$ de volumen de disolución.

\subsubsection{3.-Técnicas de observación y recuento}

Los cristales de calcita que forman los cocolitos presentan muy poca birrefringencia bajo la luz natural del microscopio óptico, pero son fácilmente visibles bajo la luz polarizada (nícoles cruzados). Dado que los cristales se ordenan en el espacio de diferentes maneras en función de la especie a la que pertenezcan, sus figuras de interferencia bajo la luz polarizada se utilizan como criterio de identificación a nivel de especie (ver capítulo 2).

Este hecho unido al pequeño tamaño de los cocolitos hace necesario el uso de, al menos, un microscopio óptico petrográfico dotado de polarizador y analizador y a ser posible, de platina giratoria y un objetivo de inmersión de 100 aumentos. Para los análisis cualitativo y cuantitativo de las asociaciones cocolitoforales contemplados en esta tesis doctoral se han utilizado un microscopio Nikon Eclipse 80-i con platina giratoria y un objetivo de inmersión de 100 aumentos que proporcionan 1000 aumentos.

\subsubsection{4.-Estimación del grado de preservación}

En el estudio de asociaciones cocolitoforales es necesario analizar el grado de preservación de los cocolitos, ya que aquellas asociaciones altamente afectadas por procesos previos y/o posteriores a su sedimentación podrían no ser representativas del ambiente en que vivieron y deben ser excluidas de las reconstrucciones paleoambientales. Para el estudio realizado en esta tesis doctoral se ha adoptado la escala propuesta por Flores y Marino (2002) que establece varios grados de preservación en base a criterios visuales. Buena $(\mathrm{G}$, good) indica que todas las estructuras de los cocolitos pueden distinguirse; moderada ( $\mathrm{M}$, moderate) indica que alguna característica morfológica ha sido alterada por disolución y/o fragmentación, pero todos los ejemplares pueden identificarse a nivel de especie; pobre (P, poor) indica disolución y/o fragmentación severas impidiendo la identificación a nivel de especie; estéril ( $\mathrm{B}$, barren) indica que no hay registro de cocolitóforos. Junto a estos criterios se han considerado los criterios intermedios "buena a moderada", "moderada a pobre" y "pobre a estéril". 


\subsubsection{5.-Recuentos}

En un primer recuento se consideraron, como mínimo, 500 especímenes por muestra. Este número asegura la representatividad de las especies mayoritarias (Fatela y Taborda, 2002). En las muestras con una elevada abundancia por campo visual este número se alcanza rápidamente, por lo que la abundancia de las especies minoritarias podría ser infravalorada. Por este motivo, todas aquellas especies que en el primer recuento no alcanzaron una abundancia relativa $>1 \%$, se consideraron nuevamente en un segundo recuento en 20 campos visuales distribuidos por toda la placa y seleccionados al azar.

\subsubsection{6.-Técnicas cuantitativas}

\section{- $\quad$ Cálculo de abundancias relativas (\%)}

Las abundancias relativas finales de las especies fueron recalculadas teniendo en cuenta las nuevas abundancias de las especies minoritarias después del segundo recuento.

- $\quad$ Cálculo de abundancias absolutas (N)

El cálculo de las abundancias absolutas de cada especie así como del total de cocolitos se realizó siguiendo el método propuesto por Flores y Sierro (1997):

$$
\mathrm{N}=\frac{\mathrm{n} * \mathrm{R}^{2} * \mathrm{~V}}{\mathrm{r}^{2} \mathrm{~g}^{*} \mathrm{v}}
$$

donde $\mathbf{N}$ es la abundancia absoluta (cocolitos ${ }^{*} \mathrm{~g}^{-1}$ ), $\mathbf{n}$ es el número de cocolitos contados por campo visual, $\mathbf{R}$ es el radio de la placa de Petri utilizada $(\mathrm{mm}), \mathbf{V}$ es el volumen de agua de la disolución inicial $(\mu \mathrm{L}), \mathbf{r}$ es el radio del campo visual del microscopio $(\mathrm{mm}), \mathbf{g}$ es el peso seco del sedimento de la disolución ( $\mathrm{g}$ ) y $\mathbf{v}$ es el volumen de muestra extraído con la micropipeta $(\mu \mathrm{L})$.

- Cálculo de las tasas de acumulación o flujos (Nannofossil Accumulation Rate $[N A R])$

Las tasas de acumulación hacen referencia al número de cocolitos acumulados por unidad de área $\left(\mathrm{cm}^{2}\right)$ y tiempo (ka) (Flores y Sierro, 1997):

\section{$\mathbf{N A R}=\mathbf{N} * \mathbf{d} * \mathbf{s}$}

donde NAR es el flujo o ratio de acumulación de cocolitos $\left(\operatorname{cocolitos} * \mathrm{~cm}^{-2} * \mathrm{ka}^{-1}\right)$, N la abundancia absoluta (cocolitos $\left.\mathrm{g}^{-1}\right)$, d la densidad de sedimento seco $\left(\mathrm{g}^{*} \mathrm{~cm}^{-3}\right)$ y s la tasa de sedimentación lineal $\left(\mathrm{cm}^{*} \mathrm{ka}^{-1}\right)$. 


\subsection{2.-Análisis de isótopos de oxígeno}

$\mathrm{El}$ análisis de isótopos estables de oxígeno $\left({ }^{16} \mathrm{O}\right.$ y $\left.{ }^{18} \mathrm{O}\right)$ se realizó en 315 muestras del testigo CEUTA10PC08 y 111 muestras del testigo HER-GC-T1. Las 315 muestras del primer testigo fueron preparadas por personal de la Universidad de Vigo, pero en ambos casos se adoptó el mismo protocolo. En todas las muestras se seleccionaron, al menos, 25 especímenes bien preservados del foraminífero planctónico Globigerina bulloides (Figura 3.10) pertenecientes a la fracción $>150 \mu \mathrm{m}$.

El procedimiento detallado adoptado para el análisis de isótopos de oxígeno puede consultarse en el Anexo III de esta tesis doctoral. Dicho procedimiento contempla los siguientes pasos más generales:

A.-Limpieza de viales

B.-Preparación de las muestras

C.-Picking y crushing

D.-Limpieza previa al análisis de isótopos estables
Figura 3.10. Imagen del foraminífero planctónico $G$. bulloides.

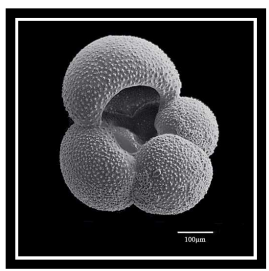

Los análisis se realizaron en la Universidad de Barcelona utilizando un espectrómetro de masas modelo SIRA equipado con un sistema de baño ácido VG Isocarb.

La relación entre ${ }^{16} \mathrm{O}$ y ${ }^{18} \mathrm{O}$ se representa mediante el ratio $\delta^{18} \mathrm{O}$, el cual viene dado en \%o y debe referenciarse a un estándar para asegurar que las estimaciones realizadas en diferentes laboratorios del mundo sean comparables:

$$
\delta{ }^{18} \mathrm{O}=\frac{\left({ }^{18} \mathrm{O} /{ }^{16} \mathrm{O}\right)_{\text {muestra }}-\left({ }^{18} \mathrm{O} /{ }^{16} \mathrm{O}\right)_{\text {estándar }}}{\left({ }^{18} \mathrm{O} /{ }^{16} \mathrm{O}\right)_{\text {estándar }}} \times 1000
$$

La calibración con la escala de estándares Vienna Pee Dee Belemnite (VPDB) (Coplen, 1996) se realizó usando el estándar NBS-19, siendo la precisión analítica superior a 0,06 \%o para el $\delta^{18} \mathrm{O}$.

Este método asume que el foraminífero sintetizó el carbonato cálcico con el que formó su concha en equilibrio con la composición isotópica del agua de mar, cuya temperatura también afectó a la composición isotópica del carbonato. De esta forma, el ratio $\delta^{18} \mathrm{O}$ aporta información sobre las variaciones en el volumen de hielo terrestre y la salinidad y temperatura del agua.

\subsection{3.-Análisis de compuestos orgánicos}

Para el análisis de compuestos orgánicos fósiles se muestrearon 114 muestras del testigo HER-GC-T1 cada 2 o $3 \mathrm{~cm}$ en los dos primeros metros del testigo desde techo y cada $5 \mathrm{~cm}$ el metro y medio restante a muro. 
El procedimiento detallado adoptado para el análisis de compuestos orgánicos, los cuales incluyen alquenonas de cadena larga, alcoholes e hidrocarbonos, está basado en el método experimental propuesto por Villanueva et al. (1997) y puede consultarse en el Anexo IV de esta tesis doctoral. Dicho procedimiento contempla los siguientes pasos generales:
A.-Pesada de la muestra
B.-Preparación del patrón
C.-Extracción con cloruro de metileno
D.-Extracción con hexano
E.-Derivatización

El procedimiento de preparación y análisis de las muestras fue realizado en el Instituto de Diagnóstico Ambiental y Estudios del Agua del CSIC de Barcelona. El análisis de las muestras se realizó con un cromatógrafo de gases Varian modelo 450, un inyector de columna en frío 1093 y un detector de ionización de llama. La fase móvil fue gas hidrógeno $\left(2,5 \mathrm{~mL}^{*} \mathrm{~min}^{-1}\right)$.

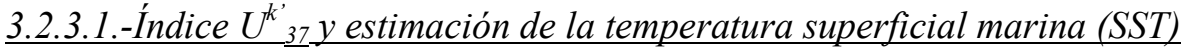

Las alquenonas son compuestos orgánicos, cetonas lineales de 37 átomos de carbono di y triinsaturadas (con 2 y 3 enlaces dobles respectivamente), sintetizados principalmente por los cocolitóforos E. huxleyi y G. oceanica (Volkman et al., 1980a; Volkman et al., 1980b; Volkman et al., 1995). El grado de insaturación de las alquenonas varía en función de la temperatura a la que se producen. Por tanto, la estimación de la abundancia de estos compuestos en los sedimentos marinos mediante el índice $\mathrm{U}^{\mathrm{k}^{\prime}}{ }_{37}$ permite el cálculo de la SST. El índice $\mathrm{U}^{\mathrm{k}^{\prime}}{ }_{37}$ se basa en las abundancias relativas de las alquenonas con 37 carbonos en su cadena $\left(\mathrm{C}_{37}\right)$ di- y triinsaturadas:

$$
\mathrm{U}^{\mathrm{k}^{\prime}}{ }_{37}=\left[\mathrm{C}_{37: 2}\right] /\left[\mathrm{C}_{37: 2}+\mathrm{C}_{37: 3}\right]
$$

La cuantificación de las alquenonas se realizó mediante la integración de sus picos en el cromatograma (Figura 3.11) obtenido para cada una de las muestras, usando el software Atlas Chromatography Data System (versión 8.2) de Thermo Electron Cooperation.

Para la estimación de la SST, el índice $\mathrm{U}^{\mathrm{k}^{\prime}}{ }_{37}$ se calibró mediante la ecuación propuesta por Müller et al. (1998):

$$
\mathrm{U}^{\mathrm{k}^{\prime}}{ }_{37}=0,033 \mathrm{~T}+0,069
$$

siendo $\mathbf{T}$ la temperatura marina a 10 metros de profundidad expresada en ${ }^{\circ} \mathrm{C}$.

\subsubsection{2.-Concentración total de alquenonas}

La concentración total de alquenonas se basa en el sumatorio de la concentración de las alquenonas $\mathrm{C}_{37}$ di- $\mathrm{y}$ tri-insaturadas: 


$$
\left[\mathrm{C}_{37}\right]=\left[\mathrm{C}_{37: 2}+\mathrm{C}_{37: 3}\right]
$$

El resultado se expresa en $\mathrm{ng}^{*} \mathrm{~g}^{-1}$ y se interpreta como un indicador del grado cualitativo de preservación de la materia orgánica en los sedimentos marinos. Así, un descenso en la concentración total de alquenonas indicará un declive en la preservación de materia orgánica.

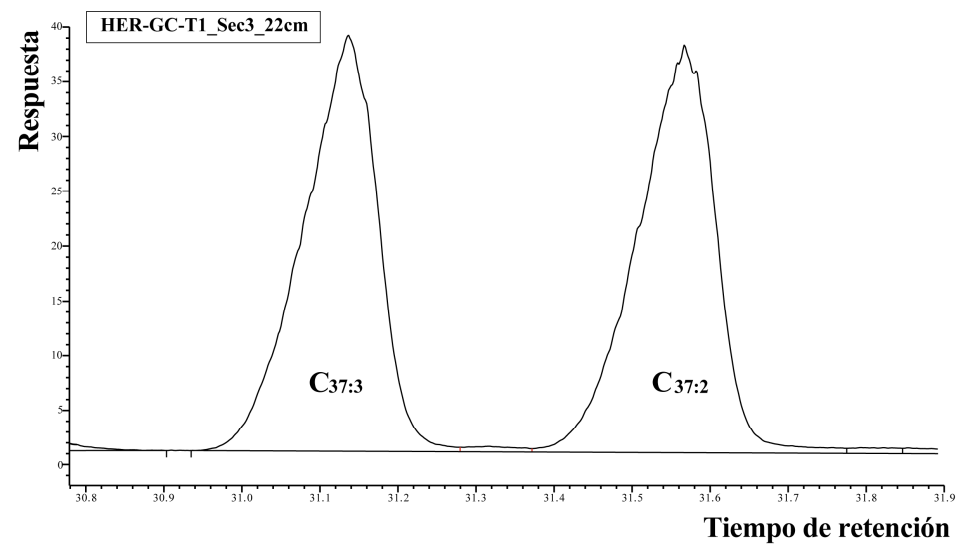

Figura 3.11. Ejemplo de los picos correspondientes a las alquenonas $\mathrm{C}_{37}$ tri- y di-insaturadas en el cromatograma obtenido para una de las muestras del testigo HER-GC-T1.

\subsubsection{3.-Índice alcohol}

El índice alcohol se basa en el ratio entre el $n$-hexacosan-1-ol $\left(\mathrm{C}_{26} \mathrm{OH}\right)$ y la suma del $\mathrm{C}_{26} \mathrm{OH}$ más el $n$-nonacosane (Martrat et al., 2007):

$$
\begin{aligned}
& \text { index } \\
& \text {-hexacosan-1-ol }
\end{aligned}=\frac{\mathrm{C}_{26} \mathrm{OH}}{\mathrm{C}_{26} \mathrm{OH}+\mathrm{C}_{29}}
$$

La cuantificación de estos compuestos se realizó, al igual que con las alquenonas, mediante la integración de sus correspondientes picos en cada uno de los cromatogramas.

El índice alcohol viene expresado en \% y es interpretado como un indicador de la resistencia de los restos de plantas vasculares a la degradación por oxigenación. Por este motivo, es utilizado como una estimación cualitativa indirecta del grado de ventilación de la cuenca por parte de las masas de agua profundas.

\subsection{4.- Técnicas estadísticas}

\subsubsection{1.-Correlación lineal simple}

La correlación lineal simple tiene como objetivo evaluar la fuerza de la relación lineal existente entre dos variables mediante el coeficiente de correlación muestral (r). Este coeficiente puede tomar valores entre $[-1,1]$, dependiendo el signo de si la relación entre las variables es 
directa $($ singo + ) o inversa (signo -). Cuanto mayor sea $\mathbf{r}$ en términos absolutos, mayor será la correlación entre las dos variables. Este análisis se realizó con la versión 3.01 del software PAST (Hammer et al., 2001).

\subsubsection{2.-Análisis de correspondencias sin tendencia (Detrended Correspondance Analyses} [DCA])

El análisis de correspondencias sin tendencia se desarrolló con el objetivo de eliminar el "efecto arco" del análisis canónico, un artefacto inherente a este último método (Lepš y Šmilauer, 2003). Sin embargo, el DCA también puede utilizarse para evaluar el tipo de respuesta de los organismos al gradiente ambiental en función de la longitud de éste. Esta respuesta puede ser de tipo lineal (si el gradiente no es muy largo) o de tipo unimodal (si el gradiente es relativamente largo). Determinar el tipo de respuesta es aconsejable para seguir después el método de ordenación (lineal o unimodal) que mejor se adecue a los datos con los que se trabaja. Este análisis se realizó con el software R (R Development Core Team, 2011) con el paquete VEGAN (Oksanen et al., 2007).

\subsubsection{3.-Análisis canónico de correspondencias (Canonical Correspondance Analyses}

\section{$[C C A])$}

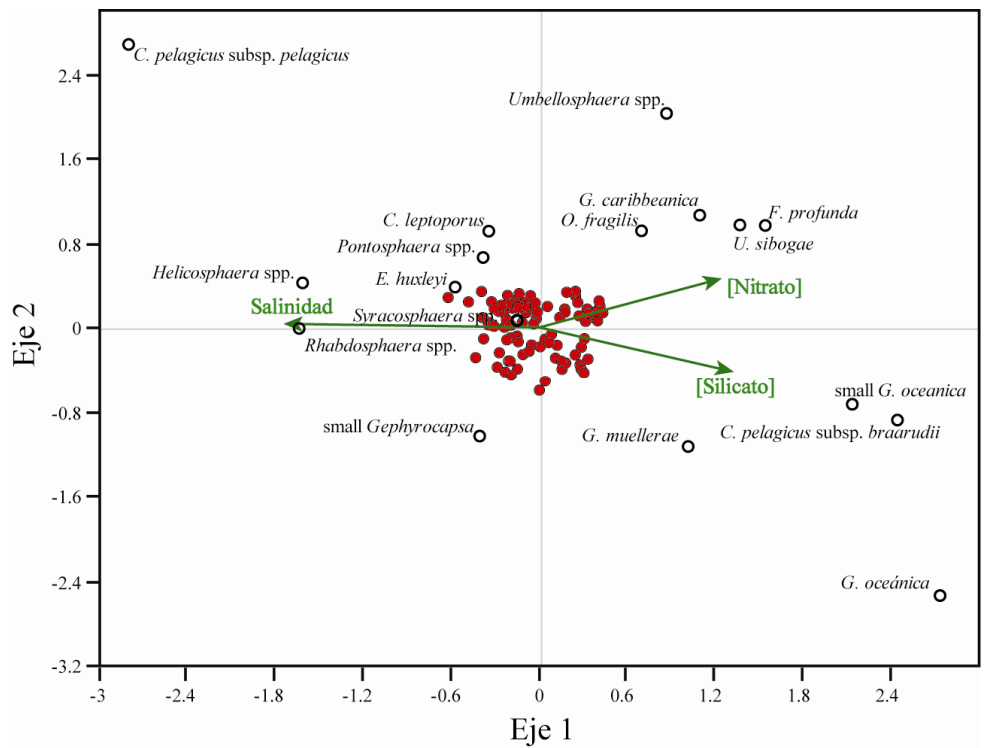

Figura 3.12. Ejemplo de un gráfico de correspondencias. Los puntos sólidos negros representan las muestras, las flechas simbolizan las tres variables consideradas en el estudio y los círculos representan cada uno de los taxones estudiados.

El análisis canónico de correspondencias es de tipo exploratorio y se resume en la obtención del gráfico de correspondencias (Figura 3.12) y su interpretación. Dicho gráfico permite representar las clases o "valores" que toman las distintas variables a comparar y concluir que los valores más próximos en esa representación son los más relacionados. Este análisis se realizó utilizando el software PAST versión 3.01 (Hammer et al., 2001). 


\subsubsection{4.-Análisis de componentes principales (Principal Component Analyses [PCA])}

El análisis de componentes principales consiste en describir la variación de las variables bajo estudio en términos de un conjunto menor de nuevas variables denominadas "Componentes Principales". Estas nuevas variables están incorreladas entre sí, pero cada una de ellas es una combinación lineal de parte de las variables originales. Las Componentes Principales son obtenidas por orden de importancia, de forma que la primera Componente Principal (CP1) incorpora la mayor cantidad posible de variación debida a las variables originales, y así sucesivamente con el resto de componentes y de información restante que aún no haya sido explicada por las CP anteriores. El objetivo de este análisis es reducir la dimensionalidad de los datos con un número de variables menor al original pero que reúna la mayor parte de su variación. Este análisis se realizó mediante el software R (R Development Core Team, 2011) con el paquete VEGAN (Oksanen et al., 2007).

\subsubsection{5.- "Forward selection"}

El análisis forward selection consiste en analizar la inclusión en el modelo de cada una de las variables así como su significatividad. Para ello, se añade repetidamente la variable con menor p-valor (siempre que éste sea menor que un valor previamente establecido; en este caso: 0,05 ), reajustando el modelo cada vez, hasta que todas las variables que se hayan incluido en el modelo tengan p-valores pequeños y sean significativas. Este método tiene un doble objetivo: i) identificar las variables correlacionadas o colineales, y ii) identificar las variables que, de entre las variables iniciales, explican por sí mismas (de manera aislada e independiente a las demás variables) una parte relevante de la varianza en la base de datos ambiental. Las variables colineales introducen información redundante y complican la interpretación de los análisis, por lo que su identificación permite excluirlas en posteriores análisis. Sólo las variables que explican información por sí mismas y son significativas por debajo de un cierto nivel de significación serán incluidas en el modelo. Este análisis se realizó mediante el software R (R Development Core Team, 2011) con el paquete VEGAN (Oksanen et al., 2007).

\subsubsection{6.-Partición de la varianza (variance partitioning)}

El análisis de partición de la varianza sirve para estimar qué proporción de la varianza observada en la base de datos biológica es explicada por cada una de las variables que resultaron significativas tras la forward selection. Este análisis se realizó con el software $\mathrm{R}$ ( $\mathrm{R}$ Development Core Team, 2011) usando el paquete VEGAN (Oksanen et al., 2007).

\subsubsection{7.-Análisis "down-core variance"}

El objetivo de este análisis es identificar qué reconstrucción, de todas las realizadas a diferentes profundidades de la columna de agua y a diferentes estaciones (verano, invierno, anual, etc.), explica una mayor cantidad de varianza observada en las asociaciones de 
cocolitóforos del testigo de sedimento. La calibración para la función de transferencia se basará en la profundidad y estación cuya reconstrucción explique una mayor cantidad de varianza y sea estadísticamente significativa. Este análisis se realizó siguiendo el método descrito por Telford et al. (2013) mediante el paquete palaeoSig v.1.1-1 (Telford, 2012) para el software R (R Development Core Team, 2011).

\subsubsection{8.-Funciones de transferencia}

Las funciones de transferencia se basan en la calibración de la relación actual entre ciertos organismos y las condiciones ambientales en las que viven. La calibración se realiza a partir de una base de datos actual (sección 3.3). Después, esta información se aplica sobre los datos de asociaciones fósiles de un testigo de sedimento con el fin de reconstruir la variabilidad de una determinada variable ambiental en el pasado. Un esquema de este proceso se muestra en la figura 3.13 .

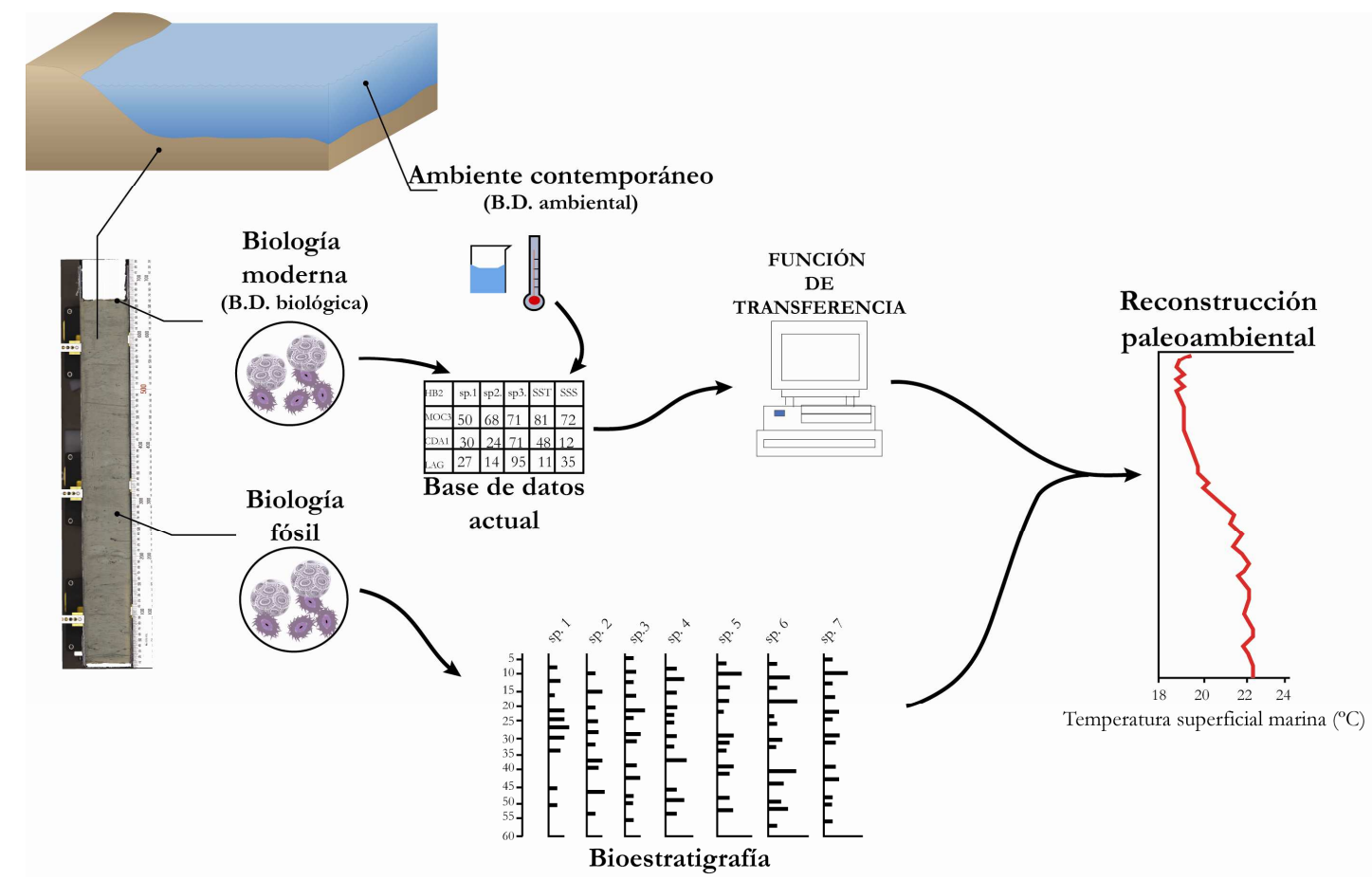

Figura 3.13. Esquema explicativo del desarrollo y aplicación de una función de transferencia. Leyenda: B.D.: base de datos.

Para el desarrollo de una función de transferencia es necesario que se cumplan dos asunciones:

i) La variable ambiental a reconstruir es, o está linealmente relacionada con, una variable ecológicamente importante del sistema.

ii) Otras variables ambientales que no sean la variable a reconstruir tienen una influencia despreciable en las variaciones observadas en las asociaciones de 
organismos, o bien, la relación de estas variables con la variable de interés ha permanecido constante a lo largo del tiempo.

En esta tesis doctoral se han empleado dos tipos de funciones de transferencia.

- $\quad$ Técnica de Análogos Modernos (Modern Analog Technique [MAT])

La Técnica de Análogos Modernos (Prell, 1985) consiste en comparar asociaciones modernas de ciertos organismos con asociaciones pertenecientes al registro sedimentario con el objetivo de encontrar las más semejantes, es decir, determinar los análogos modernos. Para aplicar este método deben cumplirse primero dos supuestos:

-Asociaciones similares de fauna o flora están ligadas a condiciones ambientales similares.

-La variable a reconstruir debe ser la más influyente en las variaciones observadas en las asociaciones de organismos o, al menos, estar correlacionada con esa variable influyente.

La "calidad" de los análogos modernos para las muestras fósiles puede calcularse estimando la distancia (disimilaridad) que separa las asociaciones pertenecientes a cada muestra moderna de las pertenecientes a cada muestra fósil (Overpeck et al., 1985).

- $\quad$ Regresión parcial por mínimos cuadrados ponderados (Weighted-averaging partialleast square regression [WA-PLS])

El método de regresión parcial por mínimos cuadrados ponderados (ter Braak y Juggins, 1993; ter Braak et al., 1993) fue concebido como una combinación del método de regresión y calibración ponderadas (adecuado para respuestas unimodales de los organismos) y del método de regresión parcial por mínimos cuadrados (indicado para respuestas lineales). La combinación de ambos métodos utiliza la estructura residual en los datos para mejorar los parámetros (u óptimos) de las especies, mejorando así el poder predictivo del método (ter Braak et al., 1993).

Ambos tipos de funciones de transferencia así como la estimación de la disimilaridad de los análogos se realizaron utilizando el software C2 (Juggins, 2007).

La calidad de la predicción que realiza cada modelo o función de transferencia puede evaluarse mediante tres coeficientes:

-Coeficiente de determinación $\left(\mathrm{R}^{2}\right)$. Éste es una estimación de la proporción de varianza explicada por el modelo. Cuanto más elevado sea este coeficiente, mayor será la proporción de varianza explicada por el modelo y por tanto, mejor su ajuste.

-Sesgo máximo (máximum bias). Es una medida de la tendencia del modelo a sobrevalorar o infraestimar los valores predichos en alguna parte del gradiente ambiental (ter Braak et al., 1993). 
-Error cuadrático medio de predicción (root-mean square error of prediction [RMSEP]). Éste es una medida de la variabilidad de la diferencia entre los valores observados y los valores predichos por el modelo. Es una estimación de la diferencia que podría darse entre los valores predichos y los reales cuando se utiliza el modelo para realizar predicciones, y viene expresado en las mismas unidades que la variable bajo estudio.

Estos tres coeficientes fueron validados mediante el método de validación cruzada conocido como bootstrap, un método robusto de remuestreo que consiste en utilizar muestras extraídas de la muestra aleatoria simple para obtener conclusiones acerca de la población de donde se extrajo dicha muestra aleatoria. Así, los datos son remuestreados repetidamente y los análisis se replican un número elevado (999 en este caso) de ciclos. Esto permite que los estimadores finales (en este caso los tres coeficientes descritos anteriormente) sean robustos ante la presencia de datos anómalos.

Además de evaluar la calidad de la predicción, es necesario realizar un diagnóstico del modelo para analizar si éste se ajusta adecuadamente a los datos. De ser así, el modelo puede utilizarse finalmente para realizar predicciones.

-El análisis de la bondad del ajuste se analiza mediante el valor de $\mathrm{R}^{2} \mathrm{y}$ de forma gráfica, representando los valores observados contra los predichos por el modelo y evaluando si éstos se aproximan a la diagonal de pendiente de valor 1 que significa predicciones perfectas.

-El análisis de homocedasticidad tiene como objetivo evaluar si la varianza de los residuos o residuales es constante. Una forma gráfica de realizarlo es mediante la representación de los residuales de los valores predichos contra los valores predichos de la variable dependiente. Esta representación debería ser más o menos constante alrededor de cero, pues de otro modo no se cumpliría la asunción de homocedasticidad sugiriendo la necesidad de transformar los datos previamente.

\subsubsection{9.-Análisis espectral REDFIT}

Este tipo de análisis espectral se basa en la aplicación del procedimiento REDFIT de Schulz y Mudelsee (2002). Es una versión más avanzada del periodograma de Lomb (Press et al., 1992), de forma que la serie temporal puede dividirse en un número de segmentos superpuestos al $50 \%$, promediando sus espectros y reduciendo así el ruido de la señal. Este análisis incluye un modelo autorregresivo de primer orden (AR1), a modo de hipótesis nula en un test de significación, para representar el espectro correspondiente al ruido de fondo rojo. De manera similar, también calcula las llamadas líneas de "falsa alarma" basadas en aproximaciones paramétricas $\left(\mathrm{chi}^{2}\right)$ y Monte Carlo, en este caso para los niveles de confianza al $95 \%$ y al $99 \%$ (Figura 3.14). El análisis espectral REDFIT se realizó con la versión 3.02 del software PAST (Hammer et al., 2001). 


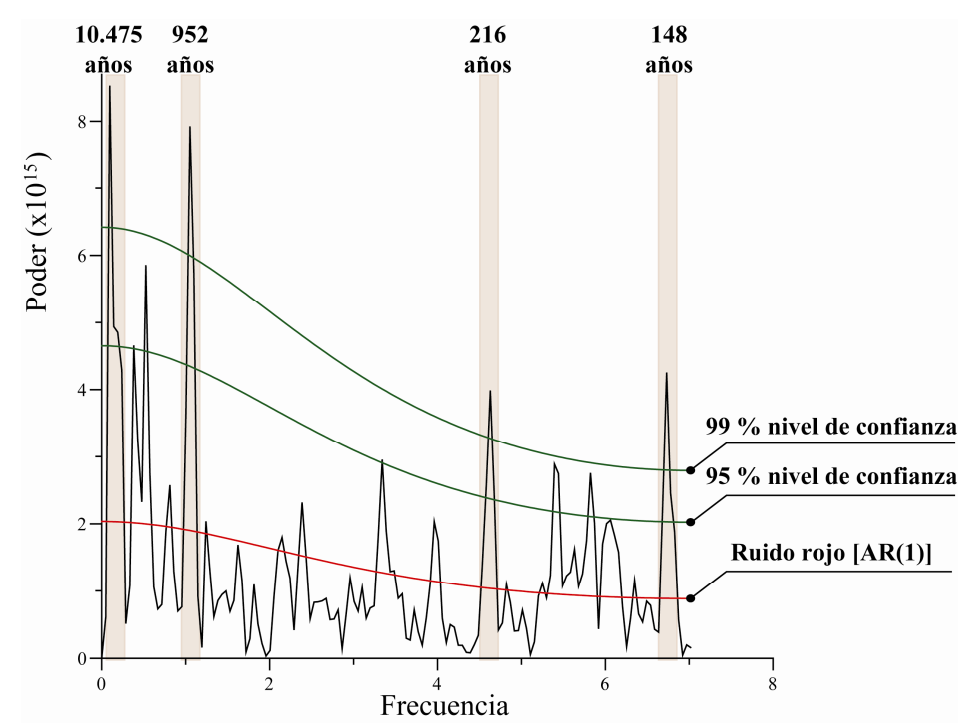

Figura 3.14. Ejemplo de periodograma obtenido mediante análisis espectral REDFIT. Las líneas verdes representan el límite inferior a partir del cual los picos resultan significativos al $95 \%$ y al $99 \%$. La línea roja representa el límite superior bajo el cual la señal es considerada ruido rojo. Las bandas beiges indican los ciclos significativos a un nivel de confianza del $99 \%$.

\section{Referencias}

Boyer TP, Antonov JI, Baranova OK, Coleman C, García HE, Grodsky A, Johnson DR, Locarnini RA, Mishonov AV, O'Brien TD, Paver CR, Reagan JR, Seidov D, Smolyar IV y Zweng MM. (2013) World Ocean Database 2013, NOAA Atlas NESDIS 72.

Bronk RC. (2008) Deposition models for chronological records. Quaternary Science Reviews 27: $42-60$.

Coplen TB. (1996) Editorial: More uncertainty than necessary. Paleoceanography 11: 369-370.

Ercilla G, Baraza J, Alonso B, Estrada F, Casas D y Farrán M. (2002) The Ceuta Drift, The Alboran Sea (Southwestern Mediterranean), Quaternary. En: Stow DAV, Pudsey CJ y Faugeres J.C. (eds) Atlas of Deep Water Contourite Systems: Modern Drifts and Ancient Series, Seismic and Sedimentary Characteristics. IGCP Publications, 155-170.

Fatela F y Taborda R. (2002) Confidence limits of species proportions in microfossil assemblages. Marine Micropaleontology 45: 169-174.

Faugères JC, Stow DAV y Imbert P. (1999) Seismic features diagnostic of contourite drifts. Marine Geology 162: 1-38.

Flores JA y Marino M. (2002) Pleistocene calcareous nannofossil stratigraphy for ODP Leg 177 (Atlantic sector of the Southern Ocean). Marine Micropaleontology 45: 191-224.

Flores JA y Sierro FJ. (1997) Revised technique for calculation of calcareous nannofossil accumulation rates. Micropaleontology 43: 321-324.

García HE, Locarnini RA, Boyer TP, Antonov JI, Baranova OK, Zweng MM, Reagan JR y Johnson DR. (2014a) World Ocean Atlas 2013, Volume 3: Dissolved Oxygen, Apparent Oxygen Utilization, and Oxygen Saturation. En: Levitus S y Mishonov A (eds) NOAA Atlas NESDIS 75, 27 pp. 
García HE, Locarnini RA, Boyer TP, Antonov JI, Baranova OK, Zweng MM, Reagan JR y Johnson DR. (2014b) World Ocean Atlas 2013, Volume 4: Dissolved Inorganic Nutrients (phosphate, nitrate, silicate). En: Levitus S y Mishonov A (eds) NOAA Atlas NESDIS 76, $25 \mathrm{pp}$.

Hammer Ø, Harper DAT y Ryan PD. (2001) PAST: Paleontological statistics software package for education and data analysis. Palaeontologia Electronica. http://palaeoelectronica.org/2001 1/past/issue1_01.htm 4: 9.

Juggins S. (2007) C2 Version 1.5 Software for ecological and palaeoecological data analysis and visualisation. En: University N (ed). Newcastle upon Tyne, UK.

Lepš J y Šmilauer P. (2003) Multivariate analysis of ecological data using CANOCO. Cambridge university press.

Locarnini, RA, AV. Mishonov, JI. Antonov, TP. Boyer, HE. García, OK. Baranova, MM. Zweng, CR. Paver, JR. Reagan, DR. Johnson, M. Hamilton, y D. Seidov (2013). World Ocean Atlas 2013, Volume 1: Temperature. En: S. Levitus y A. Mishonov (eds) NOAA Atlas NESDIS 73, $40 \mathrm{pp.}$

Müller PJ, Kirst G, Ruhland G, von Storch I y Rosell-Melé A. (1998) Calibration of the alkenone paleotemperature index $\mathrm{U}_{37}{ }^{\mathrm{K}^{\prime}}$ based on core-tops from the eastern South Atlantic and the global ocean $\left(60^{\circ} \mathrm{N}-60^{\circ} \mathrm{S}\right)$. Geochimica et Cosmochimica Acta 62 : 1757-1772.

Oksanen J, Kindt R, Legendre P, O’Hara B, Stevens MHH, Oksanen MJ y Suggests MASS. (2007) The vegan package. Community ecology package.

Overpeck JT, Webb T y Prentice IC. (1985) Quantitative interpretation of fossil pollen spectra: Dissimilarity coeffi cients and the method of modern analogs. Quaternary Research 23: $87-108$.

Prell WL. (1985) The stability of low-latitude sea-surface temperatures: An evaluation of the CLIMAP reconstruction with emphasis on the positive SST anomalies. Department of Energy. Washington, D. C., 60.

Press WH, Teukolsky SA, Vetterling WT y Flannery BP. (1992) Numerical Recipes in C. Cambridge. University Press.

R Development Core Team. (2011) R: A language and environment for statistical computing. R Foundation for Statistical Computing.

Reimer PJ, Bard E, Bayliss A, Beck JW, Blackwell PG, Bronk Ramsey C, Grootes PM, Guilderson TP, Haflidason H, Hajdas I, HattŽ C, Heaton TJ, Hoffmann DL, Hogg AG, Hughen KA, Kaiser KF, Kromer B, Manning SW, Niu M, Reimer RW, Richards DA, Scott EM, Southon JR, Staff RA, Turney CSM y van der Plicht J. (2013) IntCal13 and Marine13 Radiocarbon Age Calibration Curves 0-50,000 Years cal BP. Radiocarbon. Radiocarbon 55. 
Schlitzer R. (2014) Ocean Data View, http://odv.awi.de.

Schulz M y Mudelsee M. (2002) REDFIT: estimating red-noise spectra directly from unevenly spaced paleoclimatic time series. Computers \& Geosciences 28: 421-426.

Siani G, Paterne M, Arnold M, Bard E., Métivier B, Tisnerat N y Bassinot F. (2000) Radiocarbon reservoir ages in the Mediterranean Sea and Black Sea. Radiocarbon 42: 271-280.

Stuiver M y Reimer PJ. (1993) Extended 14C data base and revised CALIB 3.0 14C Age calibration program. Radiocarbon 35: 215-230.

Telford R. (2012) palaeoSig: significance tests of quantitative palaeoenvironmental reconstructions. R Package Version, 1.1-1.

Telford RJ, Li C y Kucera M. (2013) Mismatch between the depth habitat of planktonic foraminifera and the calibration depth of SST transfer functions may bias reconstructions. Climate of the Past 9: 859-870.

ter Braak CJF y Juggins S. (1993) Weighted averaging partial least squares regression (WAPLS): an improved method for reconstructing environmental variables from species assemblages. Hydrobiologia 269-270: 485-502.

ter Braak CJF, Juggins S, Birks HJB y van der Voet. H. (1993) Weighted averaging partial least squares regresion (WA-PLS): definition and comparison with other methods for species-environment calibration. Chapter 25. En: Patil GP y Rao CR (eds) Multivariate Environmental Statistics. North-Holland, Amsterdam.

Villanueva J, Pelejero C y Grimalt JO. (1997) Clean-up procedures for the unbiased estimation of $\mathrm{C}_{37}$ alkenone sea surface temperatures and terrigenous n-alkane inputs in paleoceanography. Journal of Chromatography A 757: 145-151.

Volkman JK, Barrerr SM, Blackburn SI y Sikes EL. (1995) Alkenones in Gephyrocapsa oceanica: Implications for studies of paleoclimate. Geochimica et Cosmochimica Acta 59: 513-520.

Volkman JK, Eglinton G, Corner EDS y Forsberg TEV. (1980b) Long-chain alkenes and alkenones in the marine coccolithophorid Emiliania huxleyi. Phytochemistry 19: 26192622 .

Volkman JK, Eglinton G, Corner EDS y Sargent JR. (1980a) Novel unsaturated straight-chain $\mathrm{C}_{37}-\mathrm{C}_{39}$ methyl and ethyl ketones in marine sediments and a coccolithophore Emiliania huxleyi. En: Douglas AG y Maxwell JR (eds) Advances in Organic Geochemistry. Pergamon, 219-227.

Zweng MM, Reagan JR, Antonov JI, Locarnini RA, Mishonov AV, Boyer TP, García HE, Baranova OK, Johnson DR, Seidov D y Biddle MM. (2013) World Ocean Atlas 2013, Volume 2: Salinity. En: Levitus S y Mishonov A (eds) NOAA Atlas NESDIS 74, 39 pp. 



$$
\text { CAPÍTULO } 4
$$





\section{4.-COCCOLITHOPHORE PRODUCTIVITY AND SURFACE WATER DYNAMICS IN THE ALBORAN SEA DURING THE LAST 25 KYR}

B. Ausín ; J-A. Flores ${ }^{\text {a }}$ F-J. Sierro $;$ M-A. Bárcena ${ }^{a}$; I. Hernández-Almeida ; G. Francés ; E. Gutiérrez-Arnillas $^{\text {c }}$; B. Martrat ${ }^{\text {d }}$ J. O. Grimalt ${ }^{\text {and I. Cacho }}{ }^{\mathrm{e}}$

${ }^{a}$ Department of Geology, University of Salamanca, La Merced, 37008 Salamanca, Spain.

${ }^{b}$ Institute of Geography and Oeschger Centre for Climate Change Research, University of Bern, Erlachstrasse 9a, CH-3012 Bern, Switzerland.

${ }^{c}$ Department of Marine Geosciences, University of Vigo, Campus As Lagoas, Marcosende, 36310 Vigo, Spain.

${ }^{\mathrm{d}}$ Department of Environmental Chemistry. Institute of Environmental Assessment and Water Research, Jordi Girona 18, 08034 Barcelona, Spain.

${ }^{e}$ Department of Stratigraphy, Paleontology and Marine Geosciences, University of Barcelona, Martí i Franquès, 08028 Barcelona, Spain.

Correspondence to: $\underline{\mathrm{b}}$ ausin@usal.es

Published in Palaeogeography, Palaeoclimatology, Palaeoecology (DOI: 10.1016/j.palaeo.2014.11.011). 


\section{Resumen}

La productividad de cocolitóforos y las dinámicas del agua superficial durante los últimos 25 ka en el mar de Alborán (Mediterráneo occidental) han sido descritas en un estudio a alta resolución de registros sedimentarios procedentes de los testigos HER-GC-T1 y CEUTA10PC08, cuyas localizaciones se caracterizan actualmente por condiciones hidrográficas diferentes. Los registros de asociaciones de cocolitóforos fósiles, isótopos de oxígeno y temperatura superficial marina $(\mathrm{SST})^{3}$ obtenida a partir de alquenonas y foraminíferos planctónicos permitieron reconstruir las propiedades del influjo de agua atlántica (AW), la cual ha probado ejercer un control de primer orden sobre las variaciones de la productividad en las zonas más cercanas al estrecho de Gibraltar. En áreas más distantes, la productividad se vio influida por otros factores locales, como la descarga fluvial y el afloramiento de agua profunda inducido por el viento o por los remolinos oceánicos. La entrada de AW fría y menos salina durante los estadiales asociados con los Eventos Heinrich 2 y 1 previno la productividad primaria. Ésta aumentó durante el Último Máximo Glacial, probablemente debido a una mayor descarga fluvial. Durante las Terminaciones la y 1b, la estratificación de la parte superior de la columna de agua no impidió el afloramiento local inducido por el viento. El Bølling-Allerød estuvo caracterizado por un incremento gradual de la productividad y el desarrollo de la Organic-Rich Layer. El Younger Dryas presenta una primera fase más fría seguida por una segunda fase más cálida y húmeda. Las diferencias en la productividad entre ambas localizaciones durante ambas fases pudieron deberse a descargas fluviales puntuales y a la variación en las propiedades de AW. La hidrografía local, como las dinámicas del giro anticiclónico occidental, cobraron mayor relevancia para determinar la productividad y sus variaciones durante el Holoceno, siendo éste el periodo más productivo.

Palabras clave: productividad de cocolitóforos, SST, isótopos de oxígeno, mar de Alborán, Último Máximo Glacial, deglaciación.

\footnotetext{
${ }^{3}$ Los acrónimos siguen la nomenclatura anglosajona por razones de consistencia con el resto del texto.
} 


\begin{abstract}
Coccolithophore productivity and surface water dynamics for the last $25 \mathrm{kyr}$ in the Alboran Sea (Western Mediterranean) are described in a study of high-resolution sedimentary records from two cores, HER-GC-T1 and CEUTA10PC08, whose locations are currently characterized by different hydrographic conditions. Fossil coccolithophore assemblages and oxygen isotopes and alkenone- and planktonic foraminifera-derived sea surface temperature (SST) records allowed a reconstruction of the properties of the inflowing Atlantic Water (AW), which have proved to be a primary control of the variations in productivity in the neighborhood of the Strait of Gibraltar. Other local factors, such as fluvial discharge, wind-induced and eddy-induced upwelling, are proposed to have influenced marine productivity in more distant areas. The entrance of cold and less saline AW during the stadials associated with Heinrich Events 2 and 1 prevented primary productivity, which increased along the Last Glacial Maximum, probably due to a greater fluvial discharge. During Terminations 1a and 1b, the upper water column was affected by stratification, although wind-induced upwelling occurred locally. The Bølling-Allerød was characterized by a gradual increase in productivity and the development of the Organic-Rich Layer. Two phases of the Younger Dryas are recognized: a first phase, which was colder, followed by a second phase, which was warmer and wetter. Differences in productivity between both locations during these two phases can be attributed to fluvial discharge and the changing properties of the AW. Local hydrography, such as the dynamics of the western anticyclonic gyre, gained greater importance in determining productivity and its variations during the Holocene, which was the most productive period.
\end{abstract}

Keywords: coccolithophore productivity, SST, oxygen isotopes, Alboran Sea, Last Glacial Maximum, deglaciation. 


\section{1.-INTRODUCTION}

The Alboran Sea is a transitional region where the Atlantic Water (AW) encounters the Mediterranean Sea. Within its small area, it exhibits most of the physical-biological interaction seen in the open ocean (García-Gorriz and Carr, 1999). Thus, current phytoplankton abundance and distribution in the Alboran Sea are governed directly by: (i) the physical, chemical and biological properties (rate of flow, SST, salinity content, nutrient concentration, etc.) of the AW that enters the Strait of Gibraltar (Navarro et al., 2011), (ii) the hydrodynamic configurations determined by the Atlantic Jet (AJ) and its two associated gyres, where vertical mixing is a primary factor (Gómez et al., 2000; Echevarría et al., 2002; Navarro et al., 2011) and (iii) upwelling processes determined by the westerly winds blowing along the south Iberian continental shelf (Sarhan et al., 2000; Navarro et al., 2011).

In this study, the variations in primary productivity during the last $25 \mathrm{kyr}$ are described by using fossil coccolithophore assemblages and placing emphasis on local changes.

Because coccolithophores are strongly influenced by nutrient availability, SST and other environmental factors, fossil coccoliths are successfully used as a widespread proxy in paleoproductivity and paleoenvironmental reconstructions (Thierstein and Young, 2004). Apart from the specific biogeography of each species, coccolithophores are sensitive to coastal currents, gyres, eddies, river discharge, and upwelling (Baumann et al., 2005; Guerreiro et al., 2013) and hence their variability in the sedimentary records allows changes in the factors that determine their local production to be tracked over time.

We have studied material from two sites: core CEUTA10PC08, located at the entrance of the Alboran Sea under the direct influence of the incoming AW, and core HER-GC-T1, located off the coast of Malaga under the influence of the semi-permanent productive cell determined by the geostrophic front of the Western Anticyclonic Gyre (WAG) (Ruiz et al., 2001) (Fig. 4.1).

Along with the high-resolution fossil coccolithophore records from these two sites, SST reconstructions (derived from $\mathrm{U}^{\mathrm{k}^{\prime}}{ }_{37^{-}}$and MAT- paleothermometers), oxygen isotopes, total concentration of $\mathrm{C}_{37}$ alkenones and the $n$-hexacosan-1-ol index were used as tracers of the environment in which coccolithophores proliferated.

Our aim was to identify the local processes determining paleoproductivity in the western Alboran basin for the last $25 \mathrm{kyr}$, together with the properties of surface (e.g. nutrient availability, temperature and salinity variability) and deep waters (ventilation) in order to gain a comprehensive understanding of the water column dynamics as a whole. 


\section{2.-STUDY AREA AND OCEANOGRAPHIC SETTING}

The Alboran Sea is the westernmost basin of the Mediterranean Sea, connected to the Atlantic Ocean through the narrow Strait of Gibraltar (Fig. 4.1a). The incoming surface flux of AW (the so-called Atlantic Jet (AJ)) describes two semi-permanent anticyclonic gyres (Fig. 4.1): the Western and the Eastern Anticyclonic Gyres (WAG and EAG, respectively) (Heburn and La Violette, 1990). The AW mixes with surface Mediterranean waters on its way to the east, forming the Modified Atlantic Water (MAW) (0-200 m) (Fig. 4.1b). At depth, Levantine Intermediate Water (LIW) (200-800 m) flows along with Western Mediterranean Deep Water (WMDW) (800-3000 m) in the opposite direction, outflowing as the Mediterranean Outflowing Water (MOW) (Fig. 4.1b). At the northern edges of the anticyclonic gyres, the mixing of AW and MAW produces a frontal system that favors vertical mixing and productive events, forming quasi-permanent areas of upwelling (García-Gorriz and Carr, 1999) (Fig. 4.1a).

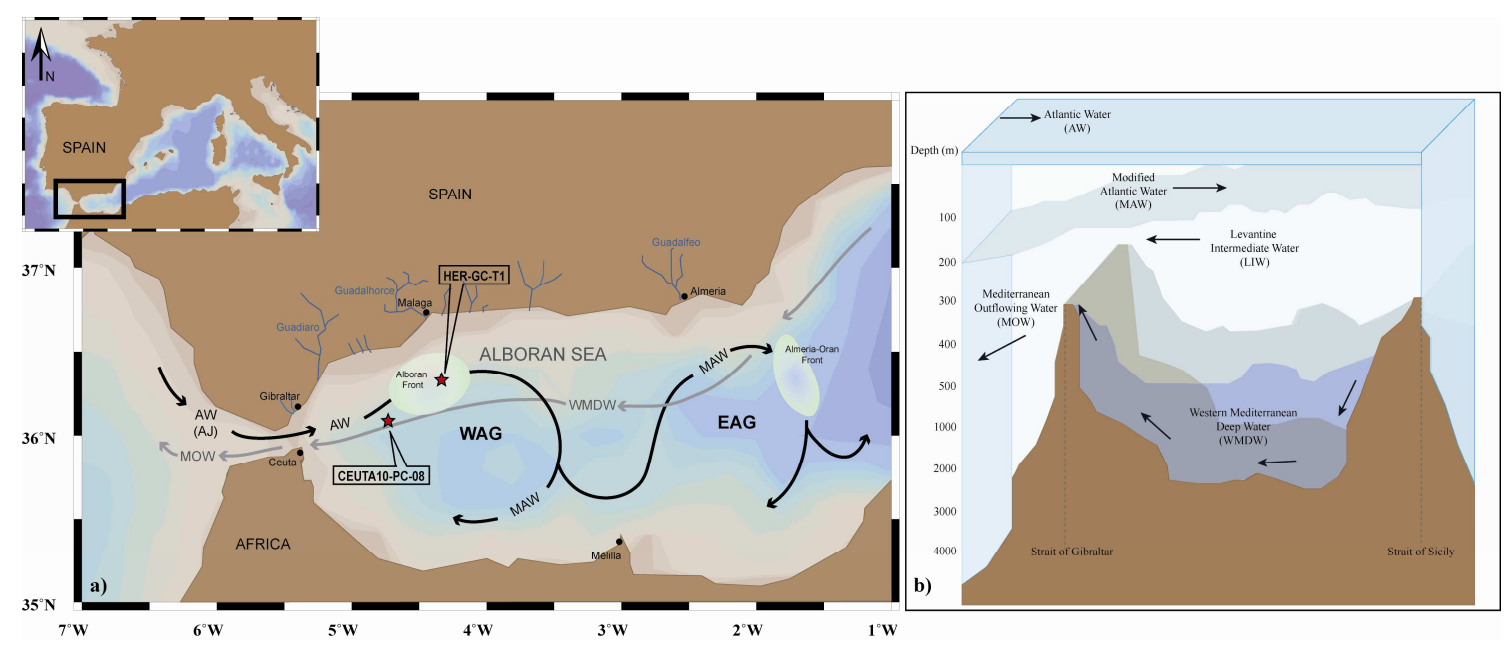

Figure 4.1. Current oceanographic setting in the Alboran Sea. a) Locations of cores CEUTA10PC08 and HER-GC-T1. Black arrows trace the general surface circulation. Gray arrows trace the general deep circulation. b) Vertical distribution of the water masses in the Western Mediterranean. AW: Atlantic Water, entering the Alboran Sea as the Atlantic Jet: AJ. MAW: Modified Atlantic Water. WMDW: Western Mediterranean Deep Water. MOW: Mediterranean Outflowing Water. WAG: Western Anticyclonic Gyre. EAG: Eastern Anticyclonic Gyre.

\section{3.-MATERIALS AND METHODS}

We analyzed two cores in the Alboran Sea: the gravity core HER-GC-T1 $\left(36^{\circ} 22^{\prime} 12^{\prime \prime} \mathrm{N}\right.$, $4^{\circ} 17^{\prime} 57^{\prime \prime} \mathrm{W}$; taken at $658.9 \mathrm{~m}$ below sea level (mbsl)), and the piston core CEUTA10PC08 $\left(36^{\circ} 12^{\prime} 22^{\prime \prime} \mathrm{N}, 4^{\circ} 52^{\prime} 3^{\prime \prime} \mathrm{W} ; 914 \mathrm{mbsl}\right.$ ) (Fig. 4.1). Core HER-GC-T1 has a length of $374 \mathrm{~cm}$ and consists of dark greenish-gray mud rich in calcareous oozes. Core CEUTA10PC08 is $648 \mathrm{~cm}$ long and is mainly composed of brown silt-mudstone. 
CAPÍTULO 4: coccolithophore productivity and surface water dynamics in the Alboran Sea during the last $25 \mathrm{kyr}$

\subsection{1.-Age model}

Table 4.1

Age model for core CEUTA-10-PC-08.

\begin{tabular}{|c|c|c|c|c|}
\hline Laboratory code & Foram type & $\begin{array}{l}\text { Depth } \\
\text { (cm) }\end{array}$ & $\begin{array}{c}\text { Radiocarbon } \\
\text { age (a) }\end{array}$ & $\begin{array}{c}\text { Calendar age } \\
\text { (a cal BP })\end{array}$ \\
\hline $\begin{array}{c}\text { CEUTA10PC08_22/ } \\
\text { Poz-56516 }^{\mathrm{a}}\end{array}$ & G. inflata & 22 & $5870 \pm 40$ & $6312 \pm 56$ \\
\hline $\begin{array}{c}\text { CEUTA10PC08_46/ } \\
\text { Poz-56517 }\end{array}$ & N. pachyderma (r.c.) & 46 & $7940 \pm 40$ & $8426 \pm 56$ \\
\hline $\begin{array}{c}\text { CEUTA10PC08_82/ } \\
\text { Poz-56518 }\end{array}$ & N. pachyderma (r.c.) & 82 & $9190 \pm 50$ & $10006 \pm 100$ \\
\hline $9977^{b}$ & N. pachyderma (r.c.) & 122 & $9900 \pm 30$ & $10885 \pm 91$ \\
\hline $9552^{b}$ & G. bulloides & 156 & $11410 \pm 30$ & $12890 \pm 79$ \\
\hline $10602^{\mathrm{b}}$ & N. pachyderma (r.c.) & 174 & $12240 \pm 70$ & $13717 \pm 107$ \\
\hline $9979^{\mathrm{b}}$ & N. pachyderma (r.c.) & 281.5 & $14270 \pm 40$ & $16818 \pm 125$ \\
\hline $9980^{b}$ & N. pachyderma (r.c.) & 323.5 & $14920 \pm 40$ & $17720 \pm 97$ \\
\hline $9981^{b}$ & N. pachyderma (r.c.) & 406 & $16910 \pm 40$ & $19936 \pm 104$ \\
\hline $9982^{b}$ & N. pachyderma (r.c.) & 452 & $18110 \pm 50$ & $21462 \pm 128$ \\
\hline $9983^{b}$ & N. pachyderma (r.c.) & 474 & $18360 \pm 50$ & $21773 \pm 106$ \\
\hline $9984^{b}$ & N. pachyderma (r.c.) & 541.5 & $20170 \pm 50$ & $23820 \pm 115$ \\
\hline $10603^{b}$ & G. bulloides & 579.5 & $20480 \pm 60$ & $24162 \pm 113$ \\
\hline $10604^{b}$ & G. bulloides & 603.5 & $21100 \pm 60$ & $24967 \pm 173$ \\
\hline $10605^{\mathrm{b}}$ & N. pachyderma (r.c.) & 615.5 & $21540 \pm 60$ & $25500 \pm 111$ \\
\hline
\end{tabular}

${ }^{\mathrm{a}}$ Refers to those ${ }^{14} \mathrm{C}$ AMS ages measured at Poznan Radiocarbon Laboratory.

${ }^{\mathrm{b}}$ Refers to those ${ }^{14} \mathrm{C}$ AMS ages measured Woods Hole Oceanographic Institution.

Fifteen radiocarbon ages for core CEUTA10PC08 (Table 4.1) and seven for core HER-GCT1 (Table 4.2) were determined on picked foraminifera shells, using the accelerator mass spectrometry (AMS) technique at the Poznan Radiocarbon Laboratory, the Center for Applied Isotope Studies of University of Georgia, and the Woods Hole Oceanographic Institution. These ${ }^{14} \mathrm{C}$ AMS ages were converted to calibrated calendar years using the OxCal 4.2 online software (Bronk, 2008) and the curve of the calibration dataset Marine13 (Reimer et al., 2013), which includes the correction of 400 years for the global marine reservoir effect. The regional difference from this global reservoir correction $(\Delta R)$ (Stuiver and Reimer, 1993) resulted in -22 82 
\pm 35 years (Siani et al., 2000) and was also considered. All dates reported here are given in calendar ages BP. The age model for core CEUTA10PC08 was based on linear interpolation between these fifteen calendar ages (Table 4.1), performed with the AnalySeries Version 1.1 (Paillard et al., 1996). This age model covers from 25.5 to $4.5 \mathrm{ka}$ (Fig. 4.2). We assigned an age of $4.5 \mathrm{ka}$ to the core top $(0 \mathrm{~cm})$ since we assumed a constant sedimentation rate between the first dated section and the core top. We are aware of the low accuracy of this assumption, but we consider that this is more reliable than assuming an age of $0 \mathrm{ka}$ at a depth of $0 \mathrm{~cm}$. The resulting average sedimentation rate was found to be $37.4 \mathrm{~cm} \mathrm{kyr}^{-1}$.

Table 4.2

Age model for core HER-GC-T1.

\begin{tabular}{|c|c|c|c|c|}
\hline $\begin{array}{c}\text { (Sample/Laboratory } \\
\text { code) }\end{array}$ & Foram Type & $\begin{array}{l}\text { Depth } \\
(\mathrm{cm})\end{array}$ & $\begin{array}{l}\text { Radiocarbon } \\
\text { age (a) }\end{array}$ & $\begin{array}{c}\text { Calendar age } \\
\text { (a cal. BP) }\end{array}$ \\
\hline SEC1_2/ Poz-53233 ${ }^{\mathrm{a}}$ & G. inflata & 2 & $440 \pm 25$ & $88 \pm 62$ \\
\hline SEC1_21/OS-87586 ${ }^{\mathrm{b}}$ & G. inflata & 21 & $1810 \pm 25$ & $1379 \pm 54$ \\
\hline SEC1_63/ Poz-53234 & G. inflata & 63 & $4175 \pm 35$ & $4284 \pm 76$ \\
\hline SEC2_17/ Poz-53235 & G. inflata & 107 & $6100 \pm 40$ & $6550 \pm 68$ \\
\hline SEC2_54/OS- $87587^{\mathrm{b}}$ & $\begin{array}{c}\text { G. inflata }+N . \\
\text { pachyderma (r.c.) }\end{array}$ & 144 & $7350 \pm 35$ & $7834 \pm 58$ \\
\hline SEC3_12/ Poz-53236 & N. pachyderma (r.c.) & 202 & $10400 \pm 60$ & $11539 \pm 162$ \\
\hline $\mathrm{SEC} 3{ }_{-} 48^{\mathrm{c}}$ & & 238 & & 14582 \\
\hline SEC3_6 $67^{\mathrm{c}}$ & & 257 & & 15217 \\
\hline $\mathrm{SEC} 3 \_77^{\mathrm{c}}$ & & 267 & & 16198 \\
\hline SEC3_92/Poz-53237 ${ }^{\mathrm{a}}$ & N. pachyderma (r.c.) & 282 & $15180 \pm 80$ & $18006 \pm 121$ \\
\hline SEC4_36 $6^{\mathrm{C}}$ & & 326 & & 21396 \\
\hline
\end{tabular}

\footnotetext{
${ }^{\mathrm{a}}$ Refers to those ${ }^{14} \mathrm{C}$ AMS ages measured at Poznan Radiocarbon Laboratory.

${ }^{\mathrm{b}}$ Refers to those ${ }^{14} \mathrm{C}$ AMS ages measured Woods Hole Oceanographic Institution.

${ }^{c}$ Refers to the tie points obtained from correlation of oxygen isotopic records.
}

The age model for HER-GC-T1 was also based on a linear interpolation between its respective seven calendar ages (Table 4.2). Three additional tie points (Table 4.2) were also considered. These were obtained by correlation between the oxygen isotopic records from this core and core CEUTA10PC08 (Fig. 4.2), since the latter has a more accurate age model. The 
resulting age model covers from 23.5 to 0.09 ka (Fig. 4.2) and the average sedimentation rate proved to be $16.6 \mathrm{~cm}^{*} \mathrm{kyr}^{-1}$.

\subsection{2.-Coccolithophore assemblage analysis}

A total of 297 samples from core CEUTA10PC08 and 143 samples from core HER-GC-T1 were considered for coccolithophore analysis, respectively providing time resolutions of 65 and 165 years, on average. Samples were prepared following the settling technique of Flores and Sierro (1997). A minimum number of 500 specimens per sample were counted and identified using a Nikon Eclipse 80-i petrographic microscope with a phase contrast device at $1000 \mathrm{x}$ magnification. A second count of 20 fields of view was performed in order to accurately estimate the abundance of rare species (those whose relative abundance was $<1 \%$ in the first count). The relative abundance (\%) and absolute abundance (number of coccoliths $\mathrm{g}^{-1}$ ) of each species were calculated for all samples. The total absolute abundance (total $\mathrm{N}$ ) was also calculated in each core as the sum of the absolute abundances of all the taxa in each sample except reworked specimens. Total $\mathrm{N}$ was interpreted as an indicator of coccolithophore productivity.

All species identified and counted in this study have been reported previously in the study area (Weaver and Pujol, 1988; Colmenero-Hidalgo et al., 2004; Álvarez et al., 2010) and are listed in Appendix A. The species Emiliania huxleyi, Gephyrocapsa aperta and Gephyrocapsa ericsonii were lumped together as "small placoliths" $(<3 \mu \mathrm{m})$ in this study owing to their common ecological significance (Winter and Siesser, 1994). Reworked specimens were taxa pertaining to older stratigraphic levels (regularly older than the Pliocene in these records), and their abundance was studied via their percentages relative to the other taxa.

According to visual criteria (little or no evidence of dissolution; diagnostic characters fully preserved) (Flores and Marino, 2002) the preservation of the coccoliths is good-to-moderate. The distal shields of some specimens were seen to have been affected by a slight degree of dissolution but this did not complicate their identification.

\subsection{3.-Oxygen stable isotopes}

Up to 20 well-preserved specimens of the planktonic foraminifer Globigerina bulloides were picked from the $>150 \mu \mathrm{m}$ size fraction in 315 samples from core CEUTA10PC08 and in 111 samples from core HER-GC-T1. Individuals were crushed, subjected to ultrasound, and cleaned with methanol before isotopic analyses were performed with a SIRA mass spectrometer at the University of Barcelona. Calibration to the Vienna Pee Dee Belemnite (VPDB) standard scale (Coplen, 1996) was accomplished using the NBS-19 standard, and analytical precision was better than $0.06 \%$ for $\delta^{18} \mathrm{O}$. 

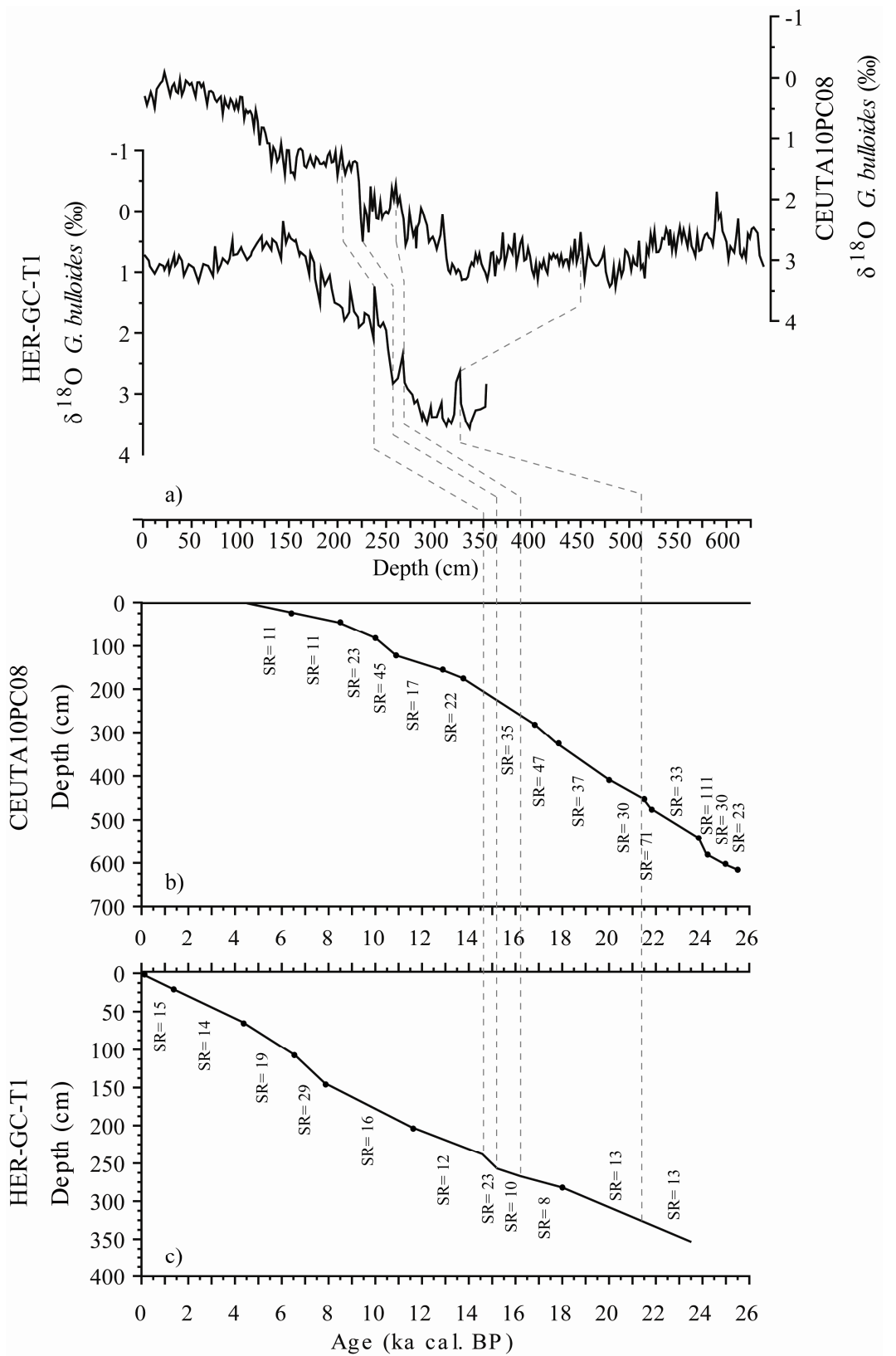

Figure 4.2. a) Oxygen isotope profiles from cores CEUTA10PC08 and HER-GC-T1. Dashed gray lines join tie points obtained from correlation of both profiles. Age-depth models from core CEUTA10PC08 (b) and core HER-GC-T1 (c). SR stands for sedimentation rate, given in $\mathrm{cm} \mathrm{kyr}^{-1}$.

\subsection{4.-Planktonic foraminifera-based SST reconstruction}

172 samples from core CEUTA10PC08 were selected and sampled every $4 \mathrm{~cm}$ for the analysis of planktonic foraminifera. Samples were first wet-sieved through a $63-\mu \mathrm{m}$ mesh, and then dry-sieved through a $150-\mu \mathrm{m}$ mesh. At least 350 specimens from a representative aliquot of the $>150 \mu \mathrm{m}$ sieved fraction were identified in each sample and the relative abundance (\%) of the species was calculated. 
Multiple SST reconstructions using planktonic foraminifera census counts were performed in order to determine the season and depth at which temperature variability appeared to be the most important for the fossil assemblage from core CEUTA10PC08. On the basis of this foraminifera census we performed a SST reconstruction using the modern analogue technique (MAT) (Prell, 1985). For the calibration we used the 862-site North Atlantic and Mediterranean planktonic foraminifera census counts compiled by Kucera et al. (2005a). Summer, winter and annual ocean temperatures for the calibration set sites were extracted at the 14 standard World Ocean Atlas (Locarnini et al., 2010) depths (between 10 and 500 m), using Ocean Data View software (Schlitzer, 2008). The calculations for MAT were performed using the C2 software (Juggins, 2003). The statistical significance of the reconstructions at different depths was tested following the procedure described by Telford et al. (2013) and performed using the palaeoSig package v.1.1-1 (Telford, 2012) for R (R Development Core Team, 2011).

\subsection{5.-Molecular biomarkers and $\mathrm{U}^{\mathrm{k}^{\prime}}{ }_{37}$-based SST}

A set of 114 samples from core HER-GC-T1 was selected for the analysis of fossil organic compounds (long chain alkenones, alcohols and hydrocarbons). A more detailed sampling was carried on the first $2 \mathrm{~m}$ of the core, sampled every $2-3 \mathrm{~cm}$, while the remaining $1.5 \mathrm{~m}$ of the core was sampled every $5 \mathrm{~cm}$. The experimental procedures used are described in Villanueva et al. (1997). Samples were analyzed with a Varian Gas Chromatograph model 450, a Cold OnColumn Injector 1093 and a Flame Ionization Detector. The carrier gas was hydrogen (2.5 $\mathrm{mL} / \mathrm{min}$ ). The identification and quantification of the $\mathrm{C}_{37}$ di- and tri-unsaturated alkenones, which are synthesized by coccolithophorid flora, allowed the calculation of the $\mathrm{U}^{\mathrm{k}^{\prime}}{ }_{37}$ index. In order to measure SST, this index was calibrated using the equation proposed by Müller et al. (1998). The total concentration of $\mathrm{C}_{37}$ alkenones $\left(\left[\mathrm{C}_{37: 2}+\mathrm{C}_{37: 3]}\right]\right)$ was also calculated. The resistance of vascular plant debris to degradation by oxygenation was examined via the relative ratio between $n$-hexacosan-1-ol $\left(\mathrm{C}_{26} \mathrm{OH}\right)$ and the sum of $\left(\mathrm{C}_{26} \mathrm{OH}\right)$ plus $n$-nonacosane $\left(\mathrm{C}_{29}\right)$ (Martrat et al., 2007). The concentration of each compound was determined using $n$ hexatriacontane as internal standard.

\subsection{6.-Statistical analyses}

The REDFIT spectral analysis method can be successfully used in unevenly sampled temporal data. PAST 3.01 software (Hammer et al., 2001) uses an implementation of the REDFIT procedure of Schulz and Mudelsee (2002). This method was applied to some of the proxies reported here in order to identify the dominant frequencies of their signals, as well as the red noise and the "false-alarm levels" (95\% and $99 \%$ ) based on parametric Chi-squared approximations at which periodicities are considered significant. 


\section{4.-RESULTS}

\subsection{1.-Coccolithophore assemblages}

Relative abundances were similar in both cores (Figs. 4.3, 4.4). Small placoliths comprise up to $90 \%$ of the fossil assemblage in some intervals while their relative abundance decreases to $40 \%$ during the stadials associated with Heinrich Events 2 and 1 (H2 and H1). Gephyrocapsa muellerae is relatively more abundant up to H1, while Gephyrocapsa oceanica and Florisphaera profunda (Fig. 4.3) begin an increasing trend from the Bølling-Allerød (B-A) onwards up-core. The relative abundance of reworked specimens is higher up to the B-A (Fig. $4.3 \mathrm{i}, \mathrm{j}$ ), showing two peaks of up to $25 \%$ during $\mathrm{H} 2$ and $\mathrm{H} 1$ in core CEUTA10PC08. E. huxleyi $(>4 \mu \mathrm{m})$, Helicosphaera spp., and Syracosphaera spp. show coeval peaks of relative abundance during $\mathrm{H} 2$ and $\mathrm{H} 1$ (Fig. 4.4). The relative abundance of Oolithotus fragilis in both cores and of Umbellosphaera irregularis in core CEUTA10PC08 peaks during Termination 1a (T1a) (Fig. $4.4 \mathrm{~g}, \mathrm{~h}, \mathrm{i})$. These species undergo an increasing trend from the onset of the YD onwards in both cores. The absolute abundance of small placoliths increases up-core in both records (Fig. 4.3a, b), except during $\mathrm{H} 2$ and $\mathrm{H} 1$, where they reach low values. G. muellerae shows an increasing trend from $9 \mathrm{ka}$ up-core in core CEUTA10PC08 (Fig. 4.3c), while in core HER-GC-T1, it shows several peaks of high values from 20 to $17.3 \mathrm{ka}$ and from 16.2 to $12.8 \mathrm{ka}$. G. oceanica shows very low values until the onset of T1a in both cores (Fig. 4.3e, f). From that time up-core, this species shows an increasing trend in core CEUTA10PC08 while several peaks of lower values are seen in core HER-GC-T1. The values of F. profunda (Fig. 4.3g, h) are very low until the onset of $\mathrm{T} 1 \mathrm{~b}$ in both cores, showing an increasing trend from $11 \mathrm{ka}$ up-core in core CEUTA10PC08 (Fig. 4.3g) and an increasing trend and large oscillations from 7.7 ka up-core in core HER-GC-T1 (Fig. 4.3h). The absolute abundance of E. huxleyi $(>4 \mu \mathrm{m})$ peaks at the onset of $\mathrm{H} 2$ in core CEUTA10PC08 and shows higher values from 19.5 to $17.5 \mathrm{ka}$ in both cores and during H1 (Fig. 4.4a, b). In core HER-GC-T1 this species also shows a peak during the YD. In core CEUTA10PC08 the absolute abundance of Helicosphaera spp. and Syracosphaera spp. (Fig. 4.4c, e, ) peaks during the H2 and the YD. From the onset of the T1b, Syracosphaera spp. display an increasing trend and high values, while Helicosphaera spp. show several peaks but no remarkable trend. In core HER-GC-T1, the profiles of both species display low values (Fig. 4.4d, f). In core CEUTA10PC08, O. fragilis and Umbellosphaera spp. (Fig. 4.4g, i) have higher absolute abundances during $\mathrm{T} 1 \mathrm{a}$ and show an increasing trend from $12.5 \mathrm{ka}$ up-core, with maximum values between 8.5 and $6.5 \mathrm{ka}$. In core HER-GC-T1, the absolute abundance of $O$. fragilis shows an increasing trend and high variability from T1a up-core (Fig. 4.4h), while Umbellosphaera spp. increases from 10 to $8.4 \mathrm{ka}$ and follows a decreasing trend from that time along the Holocene (Fig. 4.4j). 
Age (ka cal. BP)

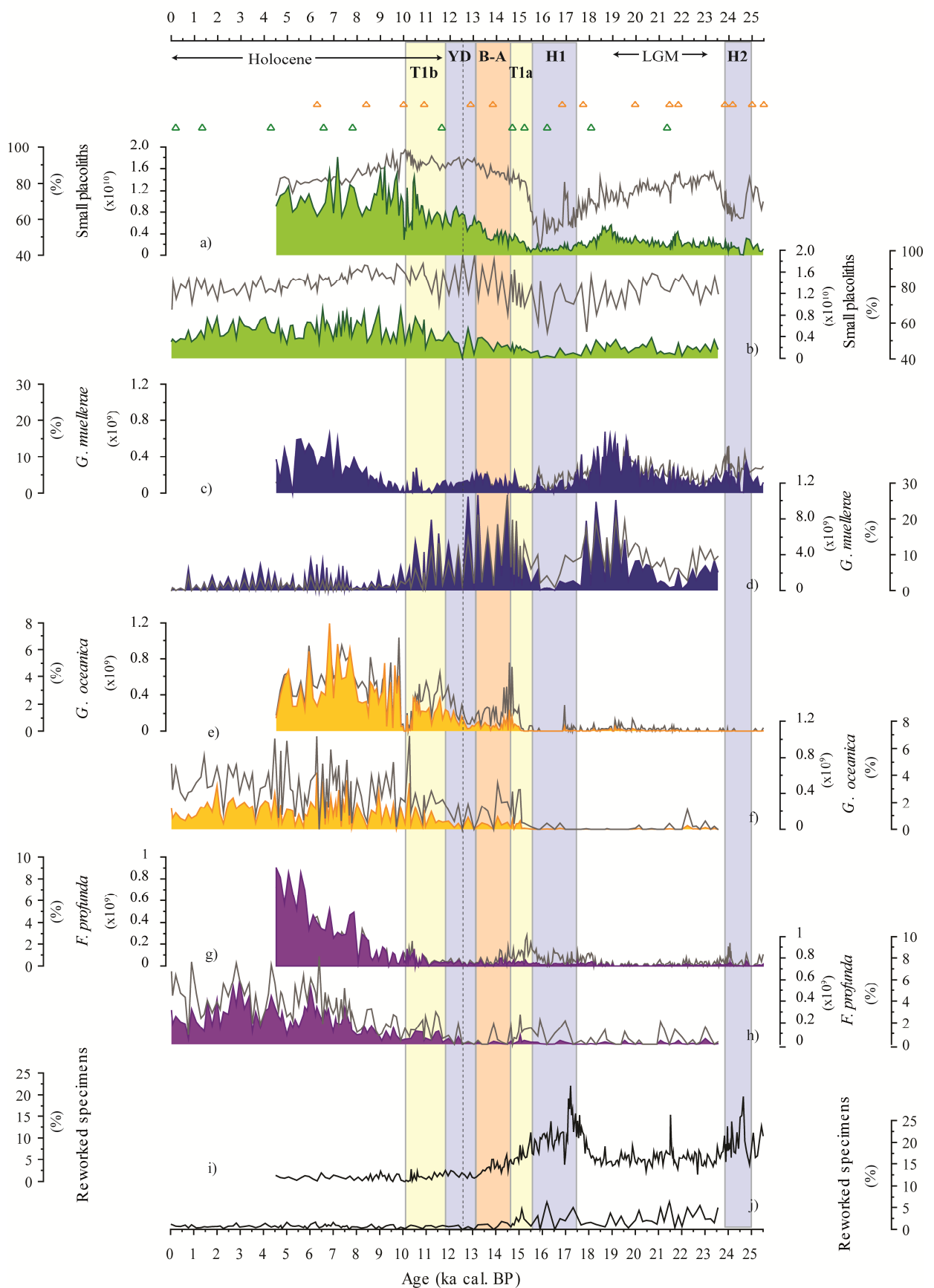

Figure 4.3. Colored logs represent absolute abundances of the main species (given in number of coccoliths $\mathrm{g}^{-1}$ ) of the nannofossil assemblages from CEUTA10PC08 (left axes) and HER-GC-T1 (right axes). The relative abundances (given in \%) of each species are represented by a dark gray solid line. H2 and H1: stadials associated with Heinrich events 2 and 1; LGM: Last Glacial Maximum; T1a: Termination 1a; B-A: Bøling-Allerød; YD: Younger Dryas. The dashed gray bar separates the YDa and YDb phases; T1b: Termination 1b. Triangles stand for age control points (kyr cal. BP) for core CEUTA10PC08 (orange) and core HER-GC-T1 (green). 
Age (ka cal. BP)

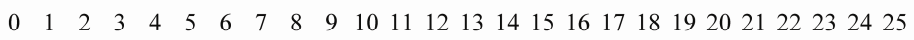

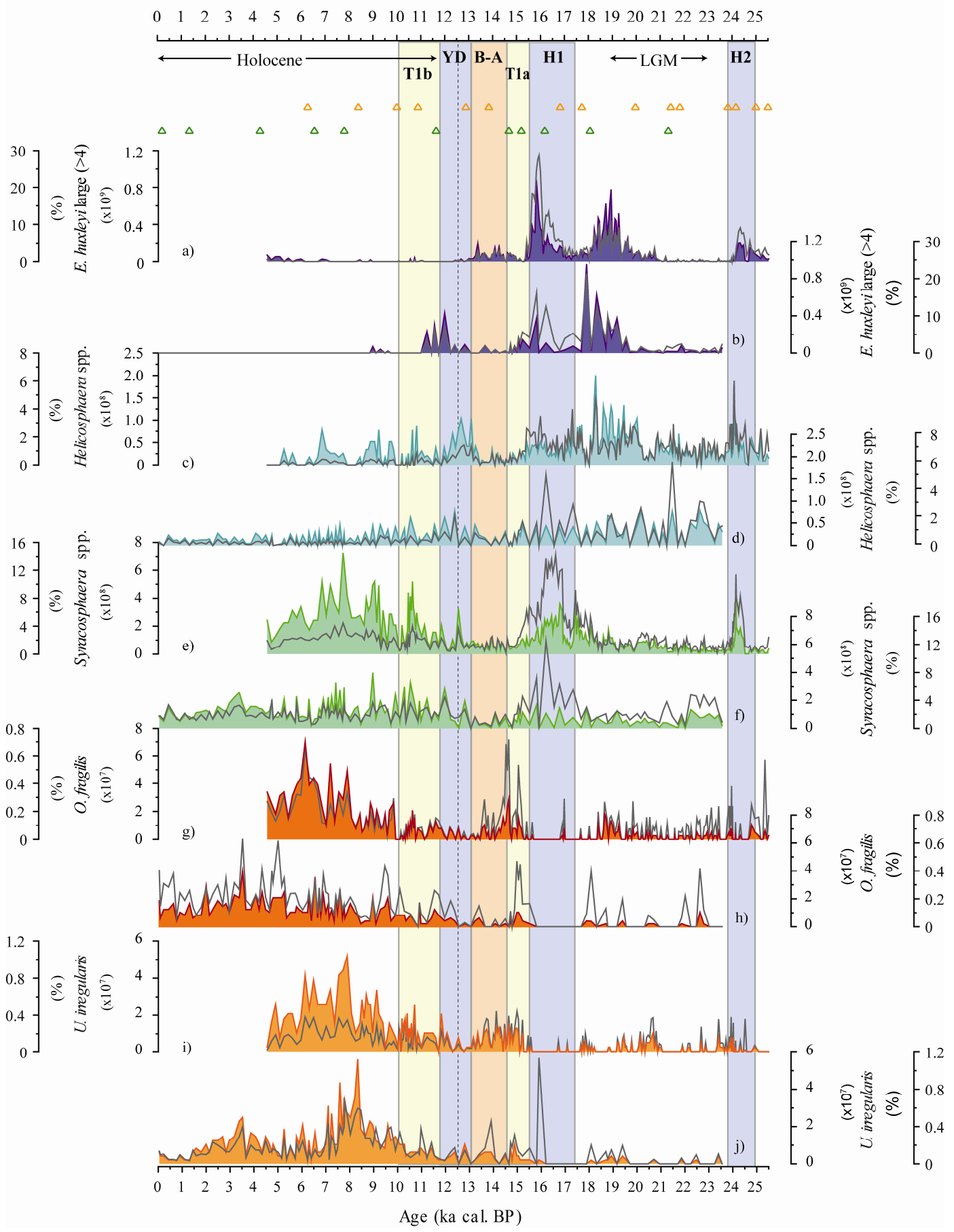

Figure 4.4. Absolute abundances (colored $\operatorname{logs}$ ) of the main nannofossil species (given in number of coccoliths $\mathrm{g}^{-1}$ ) from CEUTA10PC08 (left axes) and HER-GC-T1 (right axes). The relative abundances (given in \%) of each species are represented by a dark gray solid line. Triangles stand for age control points for core CEUTA10PC08 (orange) and core HER-GC-T1 (green).

The total N profiles of both cores (Fig. 4.5d, e) show similar general trends: low values from 25 to $15 \mathrm{ka}$ and a peak at $18.8 \mathrm{ka}$, an increasing trend and high variability from 15 to $7.5 \mathrm{ka}$, and 
high values and marked variability from $7.5 \mathrm{ka}$ up-core. It is worth noting that total $\mathrm{N}$ absolute values in core CEUTA10PC08 are double those found in core HER-GC-T1.

\subsection{2.-Oxygen Isotope Record}

Common overall patterns are observed in both records (Fig. 4.5b): higher values from 23.5 to $17.5 \mathrm{ka}$, a trend to lower values from 23.5 to $10 \mathrm{ka}$, and lower values from 10 to $4.5 \mathrm{ka}$ (minimum values being seen at $8 \mathrm{ka}$ ). The last $4.5 \mathrm{ka}$ is only available in the oxygen isotopic profile of core HER-GC-T1, which is stabilized and shows no remarkable trend.

With regard to short-term changes, a fast depletion $(0.9 \%$ ) is seen during $\mathrm{H} 2$, a period only available in core CEUTA10PC08. In addition, both records are punctuated by depletions at 21 ka (1.6\%), at $19 \mathrm{ka}$ (better seen in core CEUTA10PC08), at $16.2 \mathrm{ka}$ during the H1 (0.5\%o in core HER-GC-T1 and $0.9 \%$ in core CEUTA10PC08), and at 15 ka during T1a (1.6\%o).

\subsection{3.-Sea surface temperature (SST)}

4.4.3.1.-Planktonic foraminifera-based SST reconstruction: calibration depth and significance

Planktonic foraminifera live in a broad range of depths in the upper ocean. Therefore, the reconstruction that best explains the variability in the fossil record reflects the depth and season that most influenced the faunal composition. Telford et al. (2013) demonstrated that planktonic foraminifera-based SST reconstructions calibrated against a fixed depth may be biased if the thermal structure of the upper ocean changed over time. We assessed the most suitable calibration depth and season for use in the reconstruction by analyzing the proportion of variance in the fossil data explained by reconstructions derived for each of them (Fig. 4.6a).

In order to test the statistical significance of the SST reconstruction in core CEUTA10PC08 we used the method proposed by Telford and Birks (2011). This reconstruction is compared with 999 alternative models trained with random environmental variables. Figure 4.6a shows that the reconstruction of warm season temperatures at $10 \mathrm{~m}$ depth (Fig. 4.6c) explains $39 \%$ of the down-core variance, and indeed explains more variance than the alternative models $(95 \%$ significance level at 0.1) (Telford and Birks, 2011). These results may possibly reflect the notion that shallow-dwelling taxa bearing photosynthetic symbionts that are constrained to the upper photic-zone (e.g. G. bulloides and Globigerinoides ruber) are more abundant in the fossil assemblage, and statistically more significant in the reconstruction. The amount of variance explained by the reconstructions from core CEUTA10PC08 declines with depth (Fig. 4.6a). 


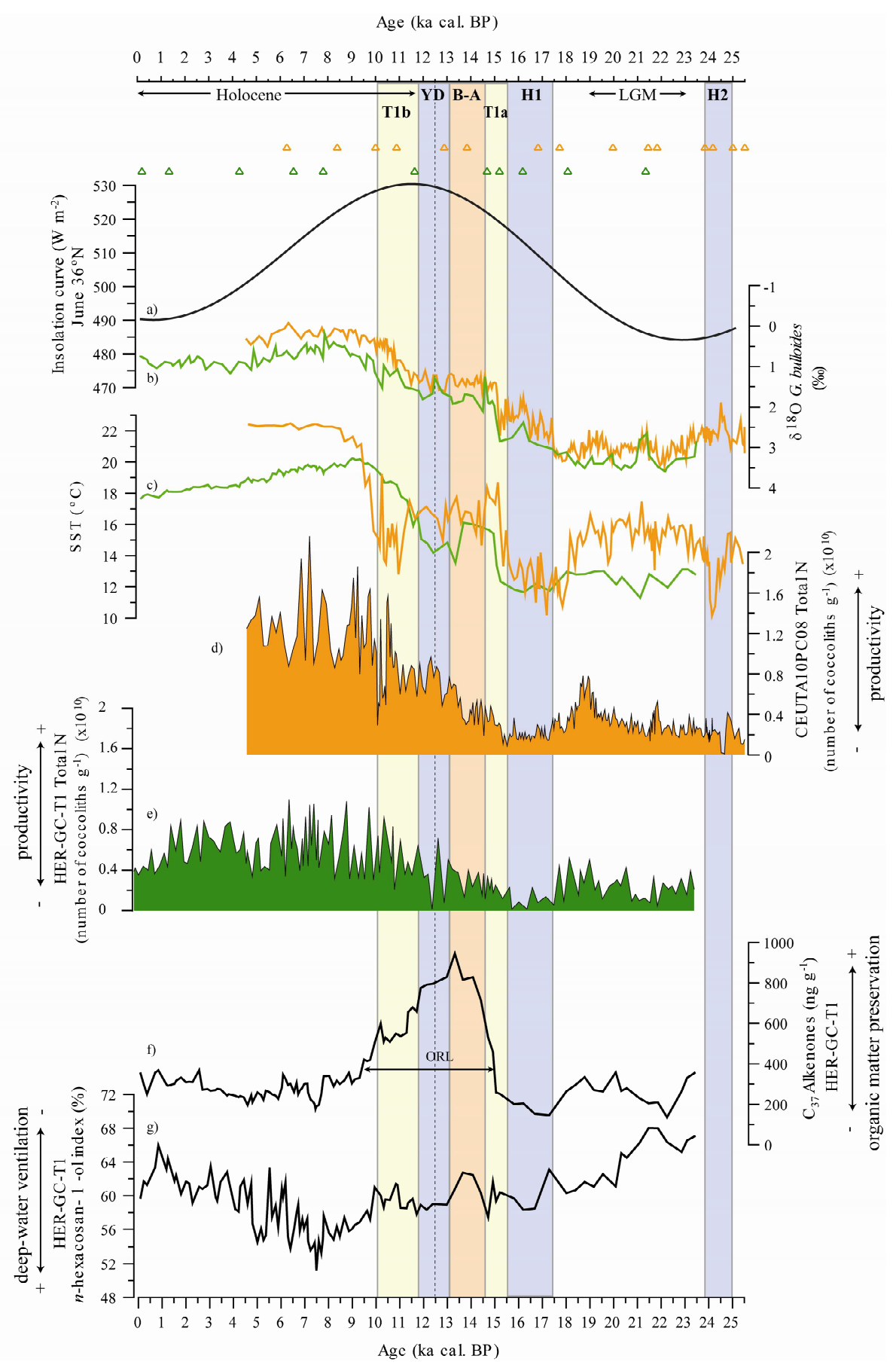

Figure 4.5. a) Insolation curve (June, $36^{\circ} \mathrm{N}$ ) (Berger, 1978). b) $\delta^{18} \mathrm{O}$ record from core CEUTA10PC08 (orange) and core HER-GC-T1 (green) (Note that the vertical axis is reversed). c) MAT-estimated SST (summer season, $10 \mathrm{~m}$ depth) of core CEUTA10PC08 (orange) and $\mathrm{U}^{\mathrm{k}^{\prime}}{ }_{37}$-estimated SST from core HERGC-T1 (green). d) Total N (total absolute abundance) from core CEUTA10PC08. e) Total N from core HER-GC-T1. f) Concentration of $\mathrm{C}_{37}$ alkenones $\left(\left[\mathrm{C}_{37: 2}+\mathrm{C}_{37: 3}\right]\right)$ from core HER-GC-T1. $\mathrm{g}$ ) $n$-hexacosan-1ol index from core HER-GC-T1. ORL: Organic-Rich Layer. Triangles stand for age control points for core CEUTA10PC08 (orange) and core HER-GC-T1 (green).

Squared chord distances between all modern assemblages were calculated to identify dissimilarities between modern and fossil assemblages. Figure $4.6 \mathrm{~b}$ shows that most of the fossil assemblages are below the $5^{\text {th }}$ percentile (minimum dissimilarity) and none are above the $10^{\text {th }}$ 
percentile (square chord distance of 21.8; non-analogue assemblages) of all distances between calibration set assemblages (Overpeck et al., 1985), indicating good analogue quality for the reconstruction. The highest dissimilarity values are seen around $10 \mathrm{ka}$ and $17 \mathrm{ka}$.
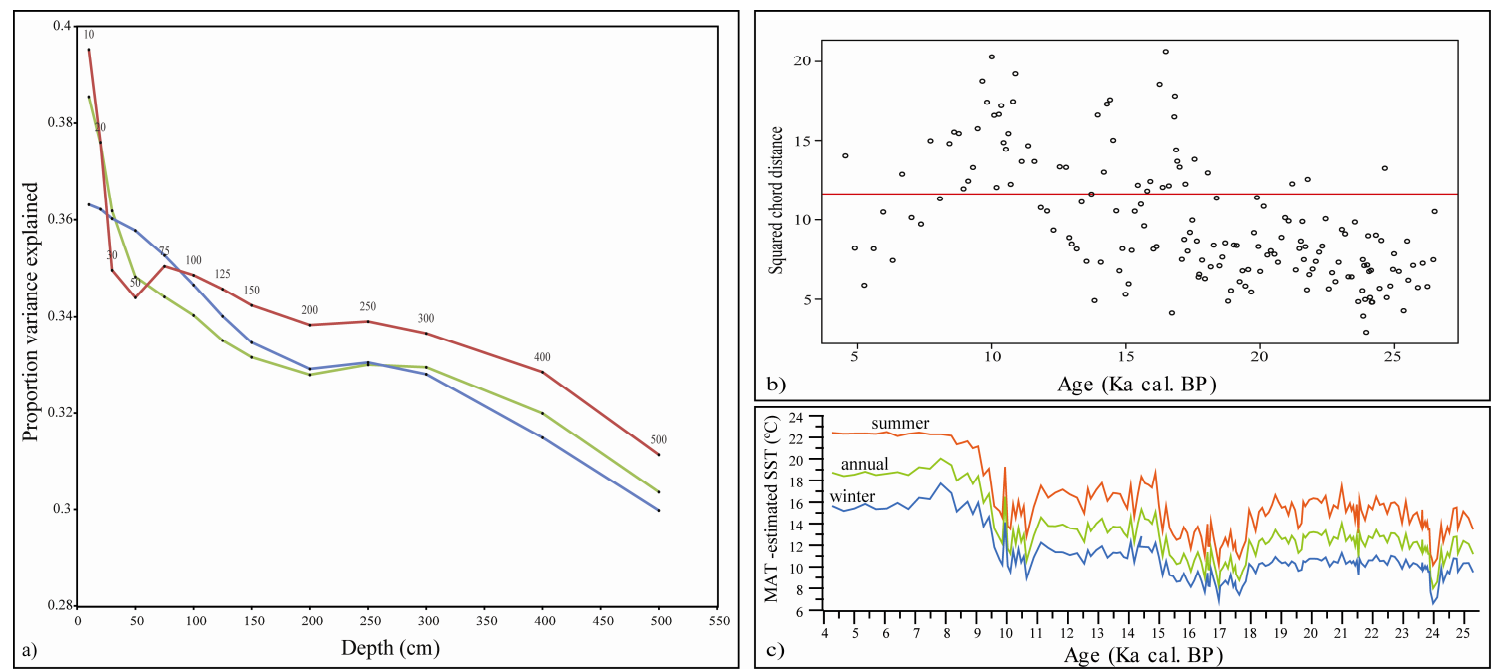

Figure 4.6. a) Proportion of variance in the fossil data explained by the reconstructions of summer (red), annual (green) and winter (blue) temperatures at different water depths. The $95 \%$ significance level is assessed at 0.1 by finding the proportion of variance explained by reconstructions of random environmental data. b) Dissimilarity between modern and core CEUTA10PC08 fossil assemblages measured by squared chord distance, plotted against age ( $\mathrm{ka}$ cal. BP). The red line represents the $5^{\text {th }}$ percentile. c) Summer, annual and winter SST reconstructions at $10 \mathrm{~m}$ water depth.

MAT-estimated summer-SST (Fig. 4.5c) dropped during $\mathrm{H} 2$ from $15.2{ }^{\circ} \mathrm{C}$ to its minimum value of $10{ }^{\circ} \mathrm{C}$ SST, and then increased and oscillated around $15.5^{\circ} \mathrm{C}$ during the LGM. Lower values are recorded during $\mathrm{H} 1$, while an increase of $6{ }^{\circ} \mathrm{C}$ is seen during T1a. SST fell by $2{ }^{\circ} \mathrm{C}$ at the onset of both the B-A and YD and fell again by $4.7^{\circ} \mathrm{C}$ at the onset of $\mathrm{T} 1 \mathrm{~b}$ followed by a rise of $8{ }^{\circ} \mathrm{C}$ from 10.5 to $9.5 \mathrm{ka}$. During the Holocene, SST oscillated slightly around $22{ }^{\circ} \mathrm{C}$.

\subsubsection{2.-Alkenone-based SST reconstruction}

The $\mathrm{U}^{\mathrm{k}^{\prime}}{ }_{37}$-estimated SST from core HER-GC-T1 (Fig. 4.5c) shows low values from $23.5 \mathrm{ka}$ to the onset of the T1a, its absolute minimum $\left(11.1^{\circ} \mathrm{C}\right)$ being seen at $21.2 \mathrm{ka}$. During T1a, SST records a fast rise of $3.6^{\circ} \mathrm{C}$ and plateaus during the first 1000 years of the $\mathrm{B}-\mathrm{A}$. SST drops by $2.5^{\circ} \mathrm{C}$ at $13.5 \mathrm{ka}$ and shows lower values during the YD followed by a rise of $5.2{ }^{\circ} \mathrm{C}$ down to 9 $\mathrm{ka}$, its maximum value being reached $\left(20.1^{\circ} \mathrm{C}\right)$ at that time. From 9 ka up-core, SST records a decreasing, smooth and constant general trend. 


\subsection{4.-Total concentration of $C_{37}$ alkenones and the $n$-hexacosan-1-ol index}

The total concentration of $\mathrm{C}_{37}$ alkenones (Fig. 4.5f) drops from 23.5 to 22.5 ka and during $\mathrm{H} 1$. A sharp increase is seen at $15 \mathrm{ka}$, peaking during the $\mathrm{B}-\mathrm{A}$, and showing high values until $9.5 \mathrm{ka}$. From that time up-core it shows several peaks of low values.

The general trend of the $n$-hexacosan-1-ol index from core HER-GC-T1 (Fig. 4.5g) decreases from 23.5 to $7.5 \mathrm{ka}$, interrupted by several peaks, such as that observed during the B-A. At 7.5 ka this profile reaches its lowest values, undergoing an increasing trend from that time up-core.

Table 4.3

Periodicities (given in years and separated by semicolons) resulting from the spectral analyses showing statistical significance at the 95 and at 99 confidence levels (\%) from core CEUTA10PC08 and core HER-GC-T1.

\begin{tabular}{|c|c|c|c|c|}
\cline { 2 - 5 } \multicolumn{1}{c|}{} & \multicolumn{2}{c|}{ Core CEUTA10PC08 } & \multicolumn{2}{c|}{ Core HER-GC-T1 } \\
\hline \hline Record & $\mathbf{9 5 \%}$ & $\mathbf{9 9} \%$ & $\mathbf{9 5 \%}$ & $\mathbf{9 9} \%$ \\
\hline small placoliths & 10000 & $308 ; 185 ; 174$ & 760 & 11700 \\
\hline G. oceanica & $185 ; 171$ & $215 ; 148$ & & 341 \\
\hline G. muellerae & & $5000 ; 179$ & & $5800 ; 428 ; 399$ \\
\hline F. profunda & & $10000 ; 176$ & 3900 & $11000 ; 360 ; 346$ \\
\hline Syracosphaera spp. & & $10000 ; 183,171$ & $7800 ; 427 ; 341$ & \\
\hline Helicosphaera spp. & 5000 & 195 & 636 & $427 ; 412$ \\
\hline Oxygen isotopes & 7000 & $180 ; 173$ & 1000 & $725 ; 416$ \\
\hline SST & & $7000 ; 303 ; 275$ & & $758 ; 534 ; 489$ \\
\hline
\end{tabular}

\subsection{5.-Spectral analyses}

Spectral analyses results are shown in Table 4.3, revealing several centennial and millennial cyclicities significant at the 0.01 and 0.05 confidence levels. Common periodicities $(\sim 175 \pm 5$ years) for the coccolithophore and the oxygen isotopic records are found in core CEUTA10PC08. This periodicity is not seen in core HER-GC-T1, where SST, oxygen isotopes and some of the coccolithophore records show a different common cyclicity ( $\sim 440 \pm 50$ years).

\section{5.-DISCUSSION}

\subsection{1.-Stadials associated with Heinrich Events 2 and 1 (H2 and H1)}

E. huxleyi $(>4 \mu \mathrm{m}$ ) peaks during H2 (only recorded by core CEUTA10PC08) (Fig. 4.4a), as well as during $\mathrm{H} 1$ in both cores (Fig. 4.4a, b), pointing to colder conditions, since it is considered a cold SST paleoindicator (Colmenero-Hidalgo et al., 2002; Colmenero-Hidalgo et al., 2004). These peaks are concurrent with a drop of $5.2^{\circ} \mathrm{C}$ during H2 (Fig. $4.5 \mathrm{c}$ ) and decreases 
of $2{ }^{\circ} \mathrm{C}$ and $1.3{ }^{\circ} \mathrm{C}$ in core CEUTA10PC08 and core HER-GC-T1, respectively, during H1 (Fig. $4.5 \mathrm{c})$, pointing to a colder SST during these events of northern ice surges.

Peaks of E. huxleyi $(>4 \mu \mathrm{m})$ match negative excursions of $\delta^{18} \mathrm{O}$ from both records (Fig. 4.5b). These isotopic depletions, not expected from the drops in SST, have been extensively explained as low-salinity surface waters entering the Alboran Sea, linked to massive North Atlantic iceberg melting (Cacho et al., 1999; Sierro et al., 2005; Melki, 2011). Smaller peaks of Syracosphaera spp. and Helicosphaera spp. are seen during these periods (Fig. 4.4c, d, e, f). The absolute abundance of Syracosphaera spp. has been reported as a fresh-water input indicator (Bukry, 1974; Weaver and Pujol, 1988), while the isolated appearance of Helicosphaera carteri has been linked to high-productivity waters in other paleoceanographic works (Giraudeau, 1992; Flores et al., 1997), records from sediment traps (Hernández-Almeida et al., 2011) and surface sediment samples (Álvarez et al., 2010). However, this latter statement is at variance with the scant absolute abundance of small placoliths (Fig. 4.3a, b), wellestablished classic indicators of high-nutrient availability (Okada and Honjo, 1973). We interpreted coeval peaks of Syracosphaera spp. and Helicosphaera spp. as being linked to the low-salinity inflowing AW. Simultaneous peaks of both species have been interpreted as less saline waters by other authors in the study area (Flores et al., 1997; Colmenero-Hidalgo et al., 2004) and in other latitudes (Álvarez et al., 2005; Flores and Sierro, 2007; Scherer et al., 2008; Maiorano et al., 2009). Thus, it is reasonable to assume that E. huxleyi ( $>4 \mu \mathrm{m})$ would have also found optimal conditions for its development not only in cold but also in low-salinity waters.

The total $\mathrm{N}$ from both cores (Fig. 4.5d, e) shows its lowest values, revealing low productivity. This is in agreement with inflowing cold and less saline waters, which are expected to have promoted stratification of the upper layers and hampered upwelling. As a consequence, only E. huxleyi ( $>4 \mu \mathrm{m})$, Syracosphaera spp. and Helicosphaera spp. would have bloomed.

The relative abundance of reworked nannofossils in core HER-GC-T1 shows several peaks but low values between $\mathrm{H} 2$ and $\mathrm{H} 1$ (Fig. 4.3j), while in core CEUTA10PC08 two striking peaks are seen at the beginning of these stadials (Fig. 4.3i). The different patterns mean that nearbottom redistribution of the material between both locations can be discarded. Downward transport from the exposed continental margin could account for the arrival of reworked material to the HER-GC-T1 core location. This mechanism proved to be useful to explain particle flux distribution in the Malaga area (Fabres et al., 2002; Masqué et al., 2003) and was invoked by Flores et al. (1997) and Colmenero-Hidalgo et al. (2004) to explain the higher relative abundance of reworked specimens during more arid periods in the study area. Although the large peaks of reworked specimens found in core CEUTA10PC08 could be also explained 94 
with this mechanism, transport and deposition by deep-water currents is another reasonable interpretation. Located farther from the continental margin, core CEUTA10PC08 was retrieved from an elongated-separated drift that is associated with a moat. Seismic stratigraphic sequence studies show that the acceleration of the deep-water currents eroded the moat, depositing the resuspended material over the core location (pers. com., Ercilla, 2014). In keeping with this hypothesis, Frigola et al. (2008) found high thermohaline circulation during the stadials associated with the Heinrich Events. Nevertheless, it is not possible to rule out downward transport from the continental margin as another likely explanation.

The $n$-hexacosan-1-ol index from core HER-GC-T1 (Fig. 4.5g) fell during H1, suggesting higher deep-water ventilation, probably due to high paleocurrent intensity during the early and late phases of the Heinrich Events (Frigola et al., 2008). From 16.2 ka this index underwent an increasing trend, pointing to a worsening of the deep-water ventilation, coeval with an isotopic depletion (Fig. 4.5c), and in line with the slowdown of the thermohaline circulation reported by Sierro et al. (2005) and Frigola et al. (2008) for several Heinrich Events attributed to the entry of less saline waters. We conclude that these conditions would have affected productivity strongly, encouraging upper water column stratification and preventing the upwelling of nutrient-rich waters during $\mathrm{H} 2$ and $\mathrm{H} 1$ (Table 4.4).

\subsection{2.-Last Glacial Maximum (LGM)}

During this period, defined between 23 and 19 ka following MARGO (Kucera et al., 2005b), the SST was higher than in the previous $\mathrm{H} 2$ and the following H1 (Fig. 4.5c). Productivity rose along the interval at both locations, as shown by higher values of total $\mathrm{N}$ (Fig. $4.5 \mathrm{~d}$, e). The absolute abundance of G. muellerae increased substantially in both records between 21 and 18 ka (Fig. $4.3 \mathrm{c}, \mathrm{d})$. Its relative abundance has been used as a cold-water indicator in the study area (Weaver and Pujol, 1988). However, it should be noted that the use of percentages to describe species variability over time must be taken with caution since they are only a relative measure. The lack of agreement among the general patterns of the absolute abundance of G. muellerae and SST profiles suggests that the former cannot be controlled only by the latter. Studies from sediment trap samples in the study area (Bárcena et al., 2004; Hernández-Almeida et al., 2011) have revealed the highest fluxes of G. muellerae during upwelling periods, controlled directly by colder and highly fertilized waters, as we proposed here. In addition, in core CEUTA10PC08 this rise is coeval with smaller peaks of small placoliths and H. carteri (Fig. 4.4c), pointing to productive waters.

From 19.5 to18 ka, peaks of E. huxleyi $(>4 \mu \mathrm{m}$ ) are seen in both records (Fig. $4.4 \mathrm{a}, \mathrm{b}$ ), possibly indicating cold and less saline surface waters, as pointed out in section 5.1., via inflowing AW and/or due to the pooling of water from fluvial discharges. Stanford et al. (2011) 
deduced meltwater release events in northern latitudes at $\sim 19 \mathrm{ka}$, although their effect in lower latitudes remains unknown. With regard to cold conditions, some authors have found that the Alboran Sea was several degrees colder than the Atlantic side of the Strait of Gibraltar and the eastern Mediterranean basin during the LGM (Hayes et al., 2005; Essallami et al., 2007; Kuhlemann et al., 2008). Owing to the local nature of this cooling, it seems unlikely that inflowing Atlantic waters could account for the bloom of cold and less saline water species, river discharges being a reasonable explanation. This mechanism is a possible nutrient source in keeping with the enhanced productivity at that time, since less saline waters are expected to favor upper water column stability and hence to hinder the upwelling of deeper waters. We therefore propose that an increase in river discharges would have occurred between 21 and 18 ka, favored by wetter conditions during the LGM (Table 4.4). This interpretation is in agreement with studies of pollen records (Fletcher and Sánchez Goñi, 2008; CombourieuNebout et al., 2009; Fletcher et al., 2010) and model-data comparisons (Kageyama et al., 2005), where the authors report more humid conditions during the LGM in the Alboran Sea as compared with the previous $\mathrm{H} 2$ and the following $\mathrm{H} 1$. The $\mathrm{C}_{37}$ alkenone profile (Fig. 4.5f) points to an increase in organic matter preservation between 21 and $18 \mathrm{ka}$, although the $n$ hexacosan-1-ol index (Fig. 4.5g) shows a trend towards increasing deep-water ventilation along this time span. This discrepancy may be due to the aforementioned high productivity that, despite better ventilation of the deep basin, could have hindered the mineralization of organic matter.

\subsection{3.-Termination 1a (T1a)}

Total $\mathrm{N}$ from both cores increases during T1a (Fig. 4.5d, e), indicating increasing productivity, although absolute values are still low. Simultaneous prominent SST rise and oxygen isotope depletion suggest warmer conditions (Fig. 4.5c) coeval with a transition from cold-water coccolithophorid flora (E. huxleyi $(>4 \mu \mathrm{m})$ ) to warm-water and oligotrophic flora: O. fragilis and Umbellosphaera spp. (McIntyre and Bé, 1967; McIntyre et al., 1970; Okada and Honjo, 1973; Colmenero-Hidalgo et al., 2004) (Fig. 4.4). Interestingly, this transition is more visible in core CEUTA10PC08 (Fig. 4.4a, g, i), where the higher SST matches increase in warm-water taxa. By contrast, in core HER-GC-T1 the peaks of these taxa are smaller (Fig. $4.4 \mathrm{~h}, \mathrm{j}$ ) and G. muellerae shows a conspicuously increasing trend (Fig. 4.3d), suggesting enhanced productivity.

Two mutually exclusive scenarios are proposed to explain the discrepancies between the CEUTA10PC08 and HER-GC-T1 sites regarding coccolithophore production: 
Table 4.4

Summary of the main results and general interpretation. $\mathrm{H} 2$ and $\mathrm{H} 1$ : stadials associated with Heinrich events 2 and 1; LGM: Last Glacial Maximum; T1a: Termination 1a; B-A: Bøling-Allerød; YD: Younger Dryas. AW: Atlantic Water; DW: Deep water; ORL: Organic-Rich Layer

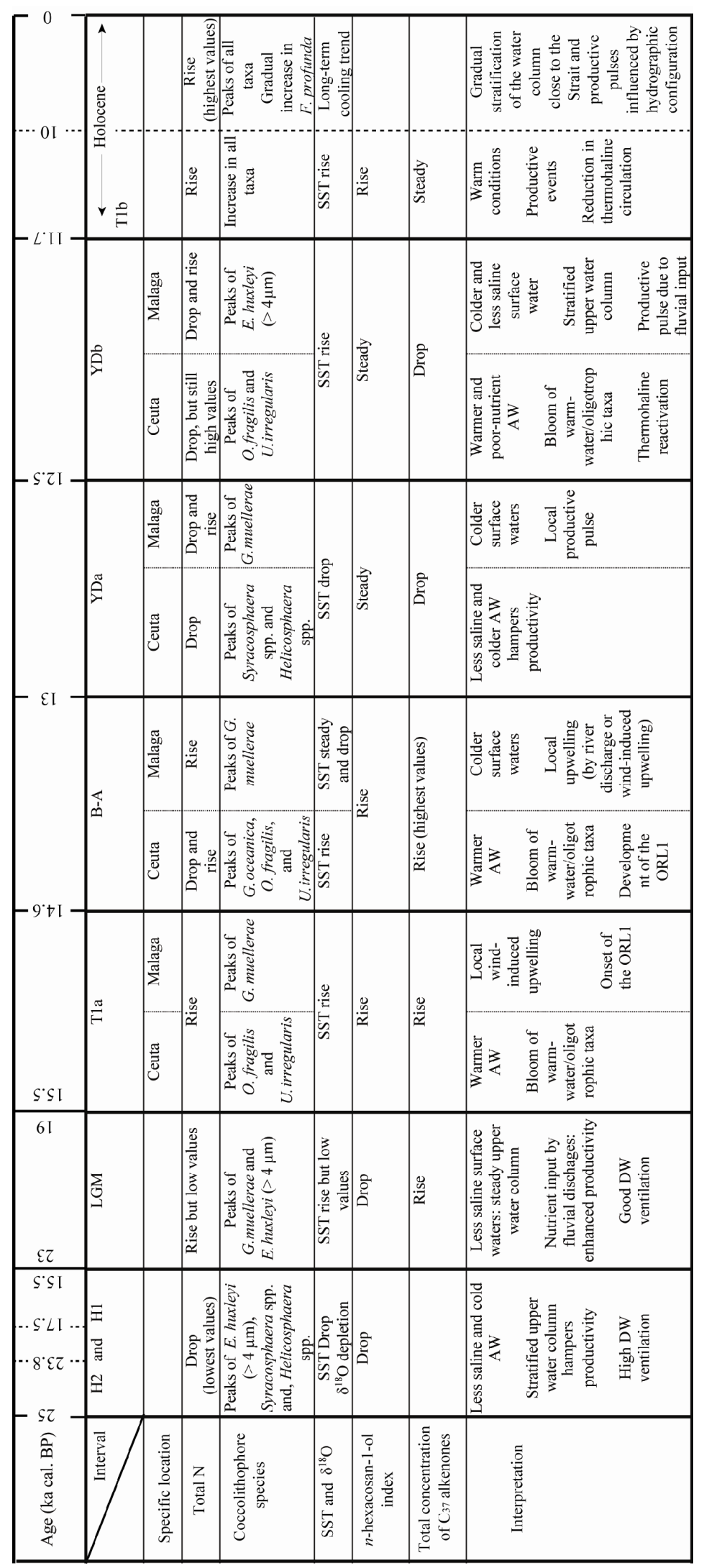


(I) Blowing westerly winds near the coast of Malaga would have induced local upwelling of cold nutrient-rich waters, favoring the blooming of G. muellerae and hampering the appearance of warm-water taxa. However, a relatively warm and poor-nutrient AW entering through the Strait would have promoted the increase in oligotrophic and warm-water taxa at the CEUTA10PC08 core location. This configuration is illustrated by the SST satellite images shown in figure 4.4 in Macías et al. (2008). Regarding this issue, it is worth mentioning that these authors found a significant negative correlation between mean nutrient concentration and the SST.

(II) A northward migration of the AJ along the northern edge of the Alboran Sea would have carried colder and nutrient-rich AW to the HER-GC-T1 core location. By contrast, at the CEUTA10PC08 core location, the warmer and impoverished-nutrient MAW would have allowed the development of warm-water and oligotrophic taxa. The satellite SST images shown in figure 4.7 in Macías et al. (2008) illustrates this hypothesis.

It is widely accepted that the contemporary hydrographic conditions with distinct geostrophic fronts separating the $\mathrm{AJ}$ from ambient Mediterranean waters occurred at around 8 ka (Rohling et al., 1995; Pérez-Folgado et al., 2003; Colmenero-Hidalgo et al., 2004), when the AW inflow rate was close to its present value. Although hypothesis II cannot be discarded, we consider that hypothesis I is a more plausible scenario (Table 4.4).

The $n$-hexacosan-1-ol index increased until 15 ka (Fig. 4.5g), indicating a weakening of the deep-water ventilation. From $15 \mathrm{ka}$, the total concentration of $\mathrm{C}_{37}$ alkenones shifts to higher values, determining the onset of the well-known organic-rich layer (ORL-1) (Sierro et al., 1998; Cacho et al., 2002; Rogerson et al., 2008) that spans 5500 years in our records. Sierro et al., (1998) and Colmenero-Hidalgo et al., (2004) described T1a as an interval with a stratified water column due to the input of deglacial waters during the sea-level rise. This scenario would have prevailed across the basin, supporting the notion that the wind-induced upwelling in the HERGC-T1 area was local.

\subsection{4.-Bølling-Allerød (B-A)}

Total $\mathrm{N}$ from both cores shows an increasing general trend, suggesting a rise in productivity along this period (Fig. 4.6d, e). In core CEUTA10PC08, G. oceanica, O. fragilis, and Umbellosphaera spp. (Figs. 4.3e, 4.4g, i) show higher absolute and relative abundances, while in core HER-GC-T1 the absolute abundance of G. muellerae increases substantially (Fig. 4.3d). In the former core, the SST persisted at the same level from 13.5 to $13 \mathrm{ka}$, while it underwent a decreasing trend in core HER-GC-T1 (Fig. 4.5c). Local factors such as river discharge or local upwelling likely promoted a SST decrease and a productivity rise at the HER-GC-T1 core 
location off the coast of Malaga, while the CEUTA10PC08 core location conditions remained steady.

The total concentration of $\mathrm{C}_{37}$ alkenones reached its maximum values along the $\mathrm{B}-\mathrm{A}$ (Fig. $4.5 \mathrm{f}$ ), indicating the high content and good preservation of organic matter that characterizes the ORL-1 (Cacho et al., 2002). This interval corresponds to high insolation (Fig. 4.5a), relatively high SST (Fig. 4.5c), and an impoverishment of the oxygen content in deep seawater as shown by the peak of $n$-hexacosan-1-ol index (Fig. 4.5g) (Table 4.4). Several authors have reported a similar scenario along with progressively humid conditions, higher river discharge, and a stable water column (Bárcena et al., 2001; Frigola et al., 2008; Fletcher et al., 2010; Rodrigo-Gámiz et al., 2011). These combined conditions, together with increasing productivity along the period, are sufficient to provide organic matter to the sediment and hamper mineralization. However, the process of ORL formation might not necessarily be so simple. Rogerson et al., (2008) studied multiproxy records from four cores in the Alboran Sea and concluded that ORL1 formation was due to a combination of reduced-density surface waters and a shoaling of the interface between intermediate and deep waters, while primary productivity was suggested to act as a secondary control.

\subsection{5.-Younger Dryas (YD)}

The cooling associated with this interval is marked by a SST drop of $2{ }^{\circ} \mathrm{C}$ in core CEUTA10PC08 and lower SST values in core HER-GC-T1 (Fig. 4.5c). In general terms, the total $\mathrm{N}$ profiles from both cores show high values pointing to high productivity (Fig. $4.5 \mathrm{~d}$, e), in agreement with previous fossil diatom and coccolithophore records (Bárcena et al., 2001; Colmenero-Hidalgo et al., 2004).

In greater detail, the SST profiles point to the occurrence of two steps during the YD: a first colder one (named here as YDa), from 13 to $12.5 \mathrm{ka}$, and a second warmer one (YDb), from 12.5 to $11.7 \mathrm{ka}$. Cool/arid and warm/humid conditions have been respectively invoked to describe the YD as a two-phase interval in the Mediterranean Sea (Cacho et al., 2002; Sbaffi et al., 2004; Combourieu-Nebout et al., 2009; Rodrigo-Gámiz et al., 2011). Coccolithophore abundances and variability also point to both phases, although differing from one core to the other, probably representing more local features than the general superimposed arid/dry and warm/humid conditions.

During YDa, core CEUTA10PC08 reflects a drop in SST (Fig. 4.5c) and in the total $\mathrm{N}$ profile (Fig. 4.5d) and coeval peaks of the absolute abundance of Syracosphaera spp. and Helicosphaera spp. (Fig. 4.4c, e). Such a scenario likely represents the entrance of colder and less saline AW, promoting a steady water column and hampering upwelling pulses. In core HER-GC-T1 total N values drop and then recover at the end of this phase (Fig. 4.5e), while $G$. 
muellerae shows high absolute abundance (Fig. 4.3d), pointing to a local productivity pulse in that area (Table 4.4).

During YDb, core CEUTA10PC08 shows higher SST values (Fig. 4.5c), small peaks of warm-water/oligotrophic taxa (Fig. $4.4 \mathrm{~g}$, i), and a small drop in the total $\mathrm{N}$ profile, although the absolute values are still high (Fig. 4.5d). This was probably due to the entrance of warmer and relatively nutrient-poor AW. Core HER-GC-T1 shows an initial drop in the total N profile,

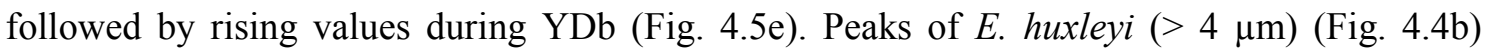
suggest local colder and less saline surface waters, expected to produce upper water column stratification. Therefore, an external nutrient source is necessary to explain the increase in local productivity. A feasible hypothesis would be the discharge of colder waters by rivers flowing into the study area (Guadalfeo River and Guadalhorce River (Fig. 4.1)). Rodrigo-Gámiz et al., (2011) described YDb as a wetter phase affected by an increase in local river discharge with increased fluvial erosion. In this situation, rivers would account for the nutrient input into the stratified upper water layer, as proposed by Bárcena et al. (2001) from the study of fossil diatom assemblages.

Although the $n$-hexacosan-1-ol index plateaus (Fig. 4.5g) indicate constant deep-water ventilation, $\mathrm{C}_{37}$ alkenones decrease gradually (Fig. 4.5f), pointing to thermohaline reactivation, as suggested by previous authors (Sierro et al., 1998; Rodrigo-Gámiz et al., 2011) (Table 4.4).

\subsection{6.-Termination $1 \mathrm{~b}(\mathrm{~T} 1 \mathrm{~b})$}

The SST reconstruction from both cores reveals opposite trends and points to a gradient of 5 ${ }^{\circ} \mathrm{C}$ between locations (Fig. 4.5c). However, similar discrepancies have been found on comparing the SST profiles obtained with both methods (alkenones and MAT) from the same core (MD45-2043 in the Alboran Sea) for this period (Pérez-Folgado et al., 2003). Moreover, comparison of $\delta^{18} \mathrm{O}$ profiles (Fig. 4.5b) does not reflect substantial offsets. These findings rule out geographical involvement and point to the different nature of the methods (i.e. ecological repercussions from the different planktonic groups employed) used to account for these discrepancies. The isotopic profiles from both cores (Fig. 4.5b) are in agreement with- $\mathrm{U}^{\mathrm{k}^{\prime}}{ }_{37^{-}}$ estimated SST (Fig. 4.5c), while the highest dissimilarity (although still good) between modern and fossil assemblages is seen for the MAT-estimated SST during this period (Fig. 4.6b). It seems likely that the SST drop recorded by the MAT-estimated SST profile would be an understimation inherent to the methodology used.

Nevertheless, total $\mathrm{N}$ profiles from both cores (Fig. $4.5 \mathrm{~d}$, e) reveal high productivity. The post-glacial sea level rise (Bard et al., 1996) is expected to have prompted a deepening of the nutricline (Colmenero-Hidalgo et al., 2004). Therefore, nutrient input from deeper water layers (i.e. upwelling or vertical mixing) can be discounted as a process responsible for maintaining 
primary productivity. Eutrophic conditions could have been promoted by a higher rate of inflow of AW enriched in nutrients. The opportunistic and eurithermal small placoliths (Okada and Wells, 1997) would bloom to the detriment of warm-water/oligotrophic taxa, despite the warmer SST (Fig. 4.5c). River runoff is another possible nutrient source, but does not exclude the previous one. Increased rainfall and more humid conditions during $\mathrm{T} 1 \mathrm{~b}$ were deduced from geochemical ratios by Frigola et al. (2008). We propose that fertilization by the inflowing AW and/or river discharge would have provided enough nutrients to maintain productivity in the photic zone despite the stratification of the water column (Table 4.4).

In agreement with this scenario, the $n$-hexacosan-1-ol index (Fig. $4.5 \mathrm{~g}$ ) increases slightly and the total concentration of $\mathrm{C}_{37}$ alkenone levels off (Fig. 4.5f), i.e., deep-water ventilation declines while organic matter preservation remains steady, in keeping with the reduction of the Mediterranean overturning during the T1b (Frigola et al., 2008).

\subsection{7.-Holocene}

The short-term variability in core HER-GC-T1 for the proxies shown here is discussed in detail in Ausín et al. (submitted for publication). In core CEUTA10PC08, the SST shows an abrupt increase until $9.5 \mathrm{ka}$ and a plateau of high values from that time onwards up-core (Fig. 4.5c). In contrast, a general cooling trend has been reported for the HER-GC-T1 core for the Holocene (Ausín et al., submitted for publication). The SST from CEUTA10PC08, which corresponds to the summer MAT-estimated SST, does not support this cooling trend. These differences may have been due to a more profound effect of seasonality during the Holocene, meaning warmer summers and cooler winters. The MAT-estimated winter and annual SST plotted in figure $4.6 \mathrm{c}$ for comparison, reveals a long-term cooling trend, supporting the notion of an amplification of seasonality. However, this interpretation disagrees with those based on pollen records, which point to cooler summers and warmer winters during this period (Fletcher and Sánchez Goñi, 2008).

Despite the high resolution, none of the proxies from core CEUTA10PC08 shows any striking variability associated with the cold and arid 8.2 ka Event (Alley et al., 1997), suggesting that its repercussions were imperceptible in the Mediterranean context (Wiersma and Renssen, 2006; Zanchetta et al., 2007).

Coccolithophore productivity in core CEUTA10PC08 shows the highest values during the Holocene (Fig. 4.5d), this being the most productive period of the last $25 \mathrm{kyr}$. The absolute abundance profiles of most taxa show the same pattern: maximum values between 7.5 and 6.5 $\mathrm{ka}$ and a decreasing trend from then onwards up-core (Fig. 4.3a, c, e and Fig. 4.4e, g, i). In contrast, $F$. profunda shows a constant increasing trend along the whole interval in core CEUTA10PC08 (Fig. 4.3g). This species inhabits the lower photic zone and blooms when the 
nutricline is located at a deeper position (Molfino and McIntyre, 1990; McIntyre and Molfino, 1996; Beaufort et al., 1997). Its gradual increasing trend along with decreasing values of other taxa suggests a growing stratification of the upper water column close to the Strait of Gibraltar, likely related to a northward migration of the AJ once in the Alboran Sea.

In terms of absolute values, coccolithophore absolute abundances show higher values in core CEUTA10PC08 than in core HER-GC-T1, not only during the Holocene but also over the last 25 kyr (Fig. 4.5d, e). Nevertheless, current productivity distribution in the Alboran Sea is greater at the HER-GC-T1 core location since it is affected by a high-productive cell off the coast of Malaga (Sarhan et al., 2000). This paradox could be explained if the increasing trend of F. profunda and decreasing trend of the other taxa seen from 7 to $4.5 \mathrm{ka}$ had persisted for the last $4.5 \mathrm{kyr}$ up-core, implying a more stratified water column characterized by low productivity at the CEUTA10PC08 core location. However, the lack of the last $4.5 \mathrm{ka}$ in core CEUTA10PC08 prevents further interpretation.

During the Holocene, the patterns shown by coccolithophore absolute abundances differ markedly between both locations (Figs. 4.3, 4.4). The WAG, depicted by a well-defined AJ that flows eastward, is one of the most notable features of the present hydrographic configuration of the Alboran Sea (Fig. 4.1), established at around 8 ka (e.g. Rohling et al., 1995). These surface dynamics entailed new factors influencing primary productivity, such as eddy-induced upwelling at the northern edge of the WAG (Sarhan et al., 2000; Ruiz et al., 2001) where HERGC-T1 is located. In turn, productivity at the CEUTA10PC08 core location would have been more influenced by the properties of the transitional AW due to its proximity to the Strait of Gibraltar. This configuration was probably responsible for the different patterns of coccolithophore absolute abundances recorded for both cores (Table 4.4).

\subsection{8.-Periodicities}

The SST and oxygen isotope records from core HER-GC-T1 reveal a common periodicity of around $740 \pm 20$ years, similar to that of $730 \pm 40$ years observed in a SST record from the Alboran Sea for the Holocene (Cacho et al., 2001). This cyclicity corresponds to the occurrence of short-term cooling events transmitted to the Mediterranean by Atlantic inflowing waters during the Holocene. Nevertheless, CEUTA10PC08 does not show such periodicity, despite being located closer to the Atlantic. Cacho et al. (2001) have reported an amplifying effect of these cooling events eastwards along the Mediterranean due to intense winds, which could account for the lack of such cyclicity in core CEUTA10PC08.

Spectral analyses do not reveal common periodicities between the two studied cores and, except for that of $740 \pm 20$ years mentioned above, to our knowledge no similar cycles have been found in nearby cores in the study area nor in the Atlantic Ocean for the last 25 kyr. Rodrigo- 
Gámiz et al. (2014) identified significant cycles of 1300, 1515, 2000, and 5000 years and secondary harmonics of 650,1087 , and 3000 years in a core from the Western Mediterranean for the last $20 \mathrm{kyr}$. These periodicities have been associated with solar activity, monsoonal regimes, orbital forcing, oceanic-atmospheric processes related to North Atlantic climate variability and African monsoon systems. Nevertheless, the authors stated that only periodicities between 500 and 7000 years have been interpreted since cyclicities outside that range could be simply a consequence of the method employed owing to the time span and the sampling interval considered, similar to those considered here. None of the above periodicities match that of $\sim$ $175 \pm 5$ years frequently found in the records of core CEUTA10PC08 or of $\sim 440 \pm 50$ years in several records of core HER-GC-T1, suggesting that the processes that took place at both locations would have been affected by several not necessarily common forcing mechanisms, possibly of local nature.

\section{6.-CONCLUSIONS}

According to the results of the present work, coccolithophores are highly sensitive to local hydrographic and environmental conditions. From the study of two oceanic cores, it may be concluded that the Atlantic water entering the Alboran Sea would have exerted primary control over productivity in the areas close to the entrance of the Strait of Gibraltar during the last 25 kyr. Their physical and biological properties (rate of inflow, nutrients, temperature and salinity) are seen to have determined the stability of the upper water column as well as the environment in which coccolithophores bloom. On its way to the east, this effect was partly diluted and hence productivity in more distant locations would also have been affected by nutrient input from river discharges, wind-induced upwelling, and specific hydrographic configurations.

As summarized in Table 4.4, coccolithophore records in combination with other paleoenvironmental proxies have allowed primary productivity variations and water column dynamics to be determined for the last $25 \mathrm{kyr}$ in the Alboran Sea:

During H2 and H1, entering cold and less saline waters prompted the stratification of the water column and prevented primary productivity.

Increased river discharge is proposed from 21 to $18 \mathrm{ka}$ to explain the cold and less saline waters in the study area as well as the enhanced productivity.

Phases T1a and T1b of the deglaciation were characterized by a stratified upper water column, although this did not hamper local wind-induced upwelling.

The $\mathrm{B}-\mathrm{A}$ is marked by a slight increase in productivity and the development of the ORL. 
The YD had two phases: a colder first phase followed by a second, warmer and wetter phase. The properties of the inflowing AW and river discharges were responsible for the differences in productivity between both locations during these two phases.

Productivity increased markedly during the Holocene. The hydrographic configuration during this period, which persists today, played an important role in its productivity and its variations.

\section{Acknowledgements}

B. Ausín is sincerely grateful to Y. González and B. Hortelano (Department of Environmental Chemistry, IDAEA-CSIC, Barcelona) for their guidance and supervision during the geochemical analyses. This study was supported by the FPU grant AP2010-2559 of the Ministry of Education of Spain awarded to B. Ausín and by the Consolider Ingenio "GRACCIE" program CSD 2007-00067, the program CGL2011-26493 and VACLIODP339, CTM200806399-C04/MAR and CTM2012-39599-03-01 projects of the Spanish Ministry of Science and Innovation.

\section{APPENDIX A. Taxonomic appendix}

The list below includes the taxa of calcareous nannoplankton in alphabetical order identified and counted in this study. Some taxa were lumped together as described in the text.

Braarudosphaera bigelowii (Gran \& Braarud 1935)

Calcidiscus leptoporus (Murray \& Blackman 1898)

Calciosolenia murrayi Gran 1912

Coccolithus pelagicus ssp. braarudii (Gaarder 1962)

Coccolithus pelagicus (Wallich 1877) Schiller 1930 ssp. pelagicus

Discosphaera tubifera (Murray \& Blackman, 1898)

Emiliania huxleyi (Lohmann 1902)

Emiliania huxleyi Type B (pujosiae) $(>4 \mu \mathrm{m})$

Florisphaera profunda (Okada \& Honjo 1973)

Gephyrocapsa aperta Kamptner 1963

Gephyrocapsa caribbeanica Boudreaux \& Hay, in Hay et al. 1967

Gephyrocapsa ericsonii McIntyre \& Bé 1967

Gephyrocapsa muellerae Bréhéret 1978

Gephyrocapsa oceanica Kamptner 1943

Helicosphaera spp., (mainly H. carteri (Wallich 1877))

Oolithotus fragilis (Lohmann 1912)

Pontosphaera spp. Schiller 1925 
Rhabdospahera clavigera Murray \& Blackman 1898

Syracosphaera spp. (mainly S. pulchra, Lohmann 1902)

Umbellosphaera spp., (mainly U. tenuis (Kamptner 1937))

Umbilicosphaera sibogae (Weber-van Bosse 1901)

\section{References}

Alley, R.B., Mayewski, P.A., Sowers, T., Stuiver, M., Taylor, K.C. and Clark, P.U. (1997) Holocene climatic instability: a prominent, widespread event 8200 yr ago. Geology 25, 483-486.

Álvarez, M.C., Flores, J.A., Sierro, F.J., Diz, P., Francés, G., Pelejero, C. and Grimalt, J. (2005) Millennial surface water dynamics in the Ría de Vigo during the last 3000 years as revealed by coccoliths and molecular biomarkers. Palaeogeography, Palaeoclimatology, Palaeoecology 218, 1-13.

Álvarez, M.C., Amore, F.O., Cros, L., Alonso, B. and Alcántara-Carrió, J. (2010) Coccolithophore biogeography in the Mediterranean Iberian margin. Revista Española de Micropaleontología 42, 359-372.

Ausín, B., Flores, J.A., Sierro, F.J., Cacho, I., Hernández-Almeida, I., Martrat, B. and Grimalt, J.O. (2014) Atmospheric patterns driving Holocene productivity in the Alboran Sea (Western Mediterranean): a multiproxy approach. The Holocene 25, 583-595.

Bárcena, M.A., Cacho, I., Abrantes, F., Sierro, F.J., Grimalt, J.O. and Flores, J.A. (2001) Paleoproductivity variations related to climatic conditions in the Alboran Sea (western Mediterranean) during the last glacial-interglacial transition: the diatom record. Palaeogeography, Palaeoclimatology, Palaeoecology 167, 337-357.

Bárcena, M.A., Flores, J.A., Sierro, F.J., Pérez-Folgado, M., Fabres, J., Calafat, A. and Canals, M. (2004) Planktonic response to main oceanographic changes in the Alboran Sea (Western Mediterranean) as documented in sediment traps and surface sediments. Marine Micropaleontology 53, 423-445.

Bard, E., Hamelin, B., Arnold, M., Montaggioni, L., Cabioch, G., Faure, G. and Rougerie, F. (1996) Deglacial sea-level record from Tahiti corals and the timing of global meltwater discharge. Nature 382, 241-244.

Baumann, K.H., Andruleit, H., Boeckel, B., Geisen, M. and Kinkel, H. (2005) The significance of extant coccolithophores as indicators of ocean water masses, surface water temperature, and palaeoproductivity: a review. Paläontologische Zeitschrift 79, 93-112.

Beaufort, L., Lancelot, Y., Camberlin, P., Cayre, O., Vincent, E., Bassinot, F. and Labeyrie, L. (1997) Insolation cycles as a major control of equatorial Indian ocean primary production. Science 278, 1451-1454. 
Berger, A. (1978) Long-termvariations of daily insolation and quaternary climatic changes. Journal of Atmospheric Sciences 35, 2362-2367.

Bronk, R.C. (2008) Deposition models for chronological records. Quaternary Science Reviews $27,42-60$.

Bukry, D. (1974) Coccoliths as paleosalinity indicators-evidence fromthe Black Sea. American Association of Petroleum Geologists 20, 353-363.

Cacho, I., Grimalt, J.O., Pelejero, C., Canals, M., Sierro, F.J., Flores, J.A. and Shackleton, N. (1999) Dansgaard-Oeschger and Heinrich event imprints in Alboran Sea paleotemperatures. Paleoceanography 14, 698-705.

Cacho, I., Grimalt, J.O., Canals, M., Sbaffi, L., Shackleton, N.J., Schönfeld, J. and Zahn, R. (2001) Variability of the western Mediterranean Sea surface temperature during the last 25,000 years and its connection with the Northern Hemisphere climatic changes. Paleoceanography 16, 40-52.

Cacho, I., Grimalt, J.O. and Canals, M. (2002) Response of the Western Mediterranean Sea to rapid climatic variability during the last 50,000 years: a molecular biomarker approach. Journal of Marine Systems 33-34, 253-272.

Colmenero-Hidalgo, E., Flores, J.A. and Sierro, F.J. (2002) Biometry of Emiliania huxleyi and its biostratigraphic significance in the Eastern North Atlantic Ocean and Western Mediterranean Sea in the last 20,000 years. Marine Micropaleontology 46, 247-263.

Colmenero-Hidalgo, E., Flores, J.A., Sierro, F.J., Bárcena, M.Á., Löwemark, L., Schönfeld, J. and Grimalt, J.O. (2004) Ocean surface water response to short-term climate changes revealed by coccolithophores from the Gulf of Cadiz (NE Atlantic) and Alboran Sea (W Mediterranean). Palaeogeography, Palaeoclimatology, Palaeoecology 205, 317-336.

Combourieu-Nebout, N., Peyron, O., Dormoy, I., Desprat, S., Beaudouin, C., Kotthoff, U. and Marret, F. (2009) Rapid climatic variability in the west Mediterranean during the last 25 000 years from high resolution pollen data. Climate of the Past 5, 503-521.

Coplen, T.B. (1996) Editorial: more uncertainty than necessary. Paleoceanography 11, 369-370. Echevarría, F., García-Lafuente, J., Bruno, M., Gorsky, G., Goutx, M., González, N., García, C.M., Gámez, F., Vargas, J.M., Picheral, M., Striby, L., Varela, M., Alonso, J.J., Reul, A., Cózar, A., Prieto, L., Sarhan, T., Plaza, F. and Jiménez-Gámez, F. (2002) Physicalbiological coupling in the Strait of Gibraltar. Deep-Sea Research II Topical Studies in Oceanography 49, 4115-4130.

Essallami, L., Sicre, M.A., Kallel, N., Labeyrie, L. and Siani, G. (2007) Hydrological changes in the Mediterranean Sea over the last 30,000 years. Geochemistry, Geophysics, Geosystems 8, Q07002. 
Fabres, J., Calafat, A., Sánchez-Vidal, A., Canals, M. and Heussner, S. (2002) Composition and spatio-temporal variability of particle fluxes in the Western Alboran Gyre, Mediterranean Sea. Journal of Marine Systems 33-34, 431-456.

Fletcher, W.J. and Sánchez Goñi, M.F. (2008) Orbital- and sub-orbital-scale climate impacts on vegetation of the western Mediterranean basin over the last 48,000 yr. Quaternary Research 70, 451-464.

Fletcher,W.J., Sánchez Goñi, M.F., Allen, J.R.M., Cheddadi, R., Combourieu-Nebout, N., Huntley, B., Lawson, I., Londeix, L.,Magri, D., Margari, V., Müller, U.C., Naughton, F., Novenko, E., Roucoux, K. and Tzedakis, P.C. (2010) Millennial-scale variability during the last glacial in vegetation records from Europe. Quaternary Science Reviews 29, 2839-2864.

Flores, J.A. and Marino, M. (2002) Pleistocene calcareous nannofossil stratigraphy for ODP Leg 177 (Atlantic sector of the Southern Ocean). Marine Micropaleontology 45, 191224.

Flores, J.A. and Sierro, F.J. (1997) Revised technique for calculation of calcareous nannofossil accumulation rates. Micropaleontology 43, 321-324.

Flores, J.A. and Sierro, F.J. (2007) Pronounced mid-Pleistocene southward shift of the Polar Front in the Atlantic sector of the Southern Ocean. Deep Sea Research II 54, 24322442.

Flores, J.A., Sierro, F.J., Frances, G., Vázquez, A. and Zamarreno, I. (1997) The last 100,000 years in the western Mediterranean: sea surface water and frontal dynamics as revealed by coccolithophores. Marine Micropaleontology 29, 351-366.

Frigola, J., Moreno, A., Cacho, I., Canals, M., Sierro, F.J., Flores, J.A. and Grimalt, J.O. (2008) Evidence of abrupt changes in WesternMediterranean Deep Water circulation during the last 50 kyr: a high-resolution marine record from the Balearic Sea. Quaternary International 181, 88-104.

García-Gorriz, E. and Carr, M.E. (1999) The climatological annual cycle of satellite-derived phytoplankton pigments in the Alboran Sea: a physical interpretation. Geophysical Research Letters 26, 2985-2988.

Giraudeau, J. (1992) Distribution of recent nannofossils beneath the Benguela system: Southwest African continental margin. Marine Geology 108, 219-237.

Gómez, F., Echevarría, F., García, C.M., Prieto, L., Ruiz, J., Reul, A., Jiménez-Gómez, F. and Varela, M. (2000) Microplankton distribution in the Strait of Gibraltar: coupling between organisms and hydrodynamic structures. Journal of Plankton Research 22, 603-617. 
Guerreiro, C., Oliveira, A., de Stigter, H., Cachão, M., Sá, C., Borges, C., Cros, L., Santos, A., Fortuño, J.M. and Rodrigues, A. (2013) Late winter coccolithophore bloom off central Portugal in response to river discharge and upwelling. Continental Shelf Research 59, 65-83.

Hammer, Ø., Harper, D.A.T. and Ryan, P.D. (2001) PAST: paleontological statistics software 656 package for education and data analysis. Palaeontologia Electronica 4, 9.

Hayes, A., Kucera,M., Kallel, N., Sbaffi, L. and Rohling, E.J. (2005). GlacialMediterranean sea surface temperatures based on planktonic foraminiferal assemblages. Quaternary Science Reviews 24, 999-1016.

Heburn, G.W. and La Violette, P.E. (1990) Variations in the structure of the anticyclonic gyres found in the Alboran Sea. Journal of Geophysical Research 95, 1599-1613.

Hernández-Almeida, I., Bárcena, M.A., Flores, J.A., Sierro, F.J., Sánchez-Vidal, A. and Calafat, A. (2011) Microplankton response to environmental conditions in the Alboran Sea (Western Mediterranean): one year sediment trap record. Marine Micropaleontology 78, $14-24$.

Juggins, S. (2003) C2 user guide. Software for Ecological and Palaeoecological Data Analysis and Visualisation. University of Newcastle, Newcastle upon Tyne, UK, p. 69.

Kageyama, M., Nebout, N.C., Sepulchre, P., Peyron, O., Krinner, G., Ramstein, G. and Cazet, J.-P. (2005). The Last Glacial Maximum and Heinrich event 1 in terms of climate and vegetation around the Alboran Sea: a preliminary model-data comparison. Comptes Rendus Geoscience 337, 983-992.

Kucera, M., Rosell-Melé, A., Schneider, R., Waelbroeck, C. and Weinelt, M. (2005a) Multiproxy approach for the reconstruction of the glacial ocean surface (MARGO). Quaternary Science Reviews 24, 813-819.

Kucera, M., Weinelt, M., Kiefer, T., Pflaumann, U., Hayes, A., Weinelt, M., Chen, M.-T., Mix, A.C., Barrows, T.T., Cortijo, E., Duprat, J., Juggins, S. and Waelbroeck, C. (2005b) Reconstruction of sea-surface temperatures from assemblages of planktonic foraminifera:multitechnique approach based on geographically constrained calibration data sets and its application to glacial Atlantic and Pacific Oceans. Quaternary Science Reviews 24, 951-998.

Kuhlemann, J., Rohling, E., Krumrei, I., Kubik, P., Ivy-Ochs, S. and Kucera, M. (2008) Regional synthesis of Mediterranean atmospheric circulation during the Last Glacial Maximum. Science 321, 1338-1340.

Locarnini, R., Mishonov, A., Antonov, J., Boyer, T., García, H., Baranova, O., Zweng, M. and Johnson, D. (2010) World ocean atlas 2009. In: Levitus, S. (Ed.), Temperature vol. 1. US Gov. Print. Off, Washington, DC (184 pp.). 
Macías, D., Bruno, M., Echevarría, F., Vázquez, A. and García, C.M. (2008) Meteorologicallyinduced mesoscale variability of the North-western Alboran Sea (southern Spain) and related biological patterns. Estuarine, Coastal and Shelf Science 78, 250-266.

Maiorano, P., Marino, M. and Flores, J.A. (2009) Thewarminterglacialmarine isotope stage 31: evidences from the calcareous nannofossil assemblages at Site 1090 (Southern Ocean). Marine Micropaleontology 71, 166-175.

Martrat, B., Grimalt, J.O., Shackleton, N.J., Abreu, L., Hutterli, M.A. and Stocker, T.F. (2007) Four climate cycles of recurring deep and surface water destabilizations on the Iberian margin. Science 317, 502-507.

Masqué, P., Fabres, J., Canals, M., Sánchez-Cabeza, J.A., Sánchez-Vidal, A., Cacho, I., Calafat, A.M. and Bruach, J.M. (2003) Accumulation rates of major constituents of hemipelagic sediments in the deep Alboran Sea: a centennial perspective of sedimentary dynamics. Marine Geology 193, 207-233.

McIntyre, A. and Bé, A.W.H. (1967) Modern coccolithophoridae of the Atlantic Ocean. Placoliths and cyrtoliths. Deep Sea Research and Oceanographic Abstracts 14, 561597.

McIntyre, A. and Molfino, B. (1996) Forcing of Atlantic equatorial and subpolar millennial cycles by precession. Science 274, 1867-1870.

McIntyre, A., Bé, A.W.H. and Roche, M.B. (1970) Modern Pacific coccolithophorida: a paleoontological thermometer. Transactions of the New York Academy of Sciences 32, $720-731$.

Melki, T. (2011) Variation of deepwater convection in the western Mediterranean Sea (Gulf of Lion) during the last $28 \mathrm{ka}$. Quaternary International 241, 160-168.

Molfino, B. and McIntyre, A. (1990) Precessional forcing of nutricline dynamics in the equatorial Atlantic. Science 249, 766-769.

Müller, P.J., Kirst, G., Ruhland, G., von Storch, I. and Rosell-Melé, A. (1998) Calibration of the alkenone paleotemperature index $\mathrm{U}_{37}{ }^{\mathrm{K}^{\prime}}$ based on core-tops from the eastern South Atlantic and the global ocean $\left(60^{\circ} \mathrm{N}-60^{\circ} \mathrm{S}\right)$. Geochimica et Cosmochimica Acta 62 , $1757-1772$.

Navarro, G., Vázquez, Á., Macías, D., Bruno, M. and Ruiz, J. (2011) Understanding the patterns of biological response to physical forcing in the Alborán Sea (western Mediterranean). Geophysical Research Letters 38, L23606.

Okada, H. and Honjo, S. (1973) The distribution of oceanic coccolithophorids in the Pacific. Deep Sea Research and Oceanographic Abstracts 20, 355-374. 
Okada, H. and Wells, P. (1997) Late quaternary nannofossil indicators of climate change in two deep-sea cores associated with the Leeuwin current off Western Australia. Palaeogeography, Palaeoclimatology, Palaeoecology 131, 413-432.

Overpeck, J., Webb III, T. and Prentice, I. (1985) Quantitative interpretation of fossil pollen spectra: dissimilarity coefficients and the method of modern analogs. Quaternary Researc. 23, 87-108.

Paillard, D., Labeyrie, L.and Yiou, P. (1996) Macintosh program performs time-series analysis. EOS Transactions American Geophysical Union 77, 379.

Pérez-Folgado, M., Sierro, F.J., Flores, J.A., Cacho, I., Grimalt, J.O., Zahn, R. and Shackleton, N., (2003) WesternMediterranean planktonic foraminifera events and millennial climatic variability during the last 70 kyr. Marine Micropaleontology 48, 49-70.

Prell, W.L. (1985) Stability of low-latitude sea-surface temperatures: an evaluation of the CLIMAP reconstruction with emphasis on the positive SST anomalies. Technical Report 25 (DOE/ER/60167-1; Other: ON: DE85016190 United StatesOther: ON: DE85016190Wed Feb 06 21:52:10 EST 2008NTIS, PC A04/MF A01; 1.ERA-10042946; EDB-85-134447English, Washington D.C.).

R Development Core Team (2011) R: A Language and Environment for Statistical Computing. R Foundation for Statistical Computing.

Reimer, P.J., Bard, E., Bayliss, A., Beck, J.W., Blackwell, P.G., Bronk Ramsey, C., Grootes, P.M., Guilderson, T.P., Haflidason, H., Hajdas, I., Hatté, C., Heaton, T.J., Hoffmann, D.L., Hogg, A.G., Hughen, K.A., Kaiser, K.F., Kromer, B., Manning, S.W., Niu, M., Reimer, R.W., Richards, D.A., Scott, E.M., Southon, J.R., Staff, R.A., Turney, C.S.M. and van der Plicht, J. (2013) IntCal13 and Marine13 radiocarbon age calibration curves 0-50,000 years cal BP. Radiocarbon 55, 1869-1887.

Rodrigo-Gámiz, M., Martínez-Ruiz, F., Jiménez-Espejo, F.J., Gallego-Torres, D., NietoMoreno, V., Romero, O. and Ariztegui, D. (2011) Impact of climate variability in the western Mediterranean during the last 20,000 years: oceanic and atmospheric responses. Quaternary Science Reviews 30, 2018-2034.

Rodrigo-Gámiz, M., Martínez-Ruiz, F., Rodríguez-Tovar, F.J., Jiménez-Espejo and F.J., PardoIgúzquiza, E. (2014) Millennial- to centennial-scale climate periodicities and forcing mechanisms in the westernmost Mediterranean for the past 20,000 yr. Quaternary Research 81, 78-93.

Rogerson, M., Cacho, I., Jiménez-Espejo, F., Reguera, M.I., Sierro, F.J., Martínez-Ruiz, F., Frigola, J. and Canals, M. (2008) A dynamic explanation for the origin of the western Mediterranean organic-rich layers. Geochemistry, Geophysics, Geosystems 9, Q07U01. 
Rohling, E.J., Den Dulk, M., Pujol, C. and Vergnaud-Grazzini, C. (1995) Abrupt hydrographic change in the Alboran Sea (western Mediterranean) around 8000 yrs BP. Deep Sea Research Part I: Oceanographic Research Papers 42, 1609-1619.

Ruiz, J., Echevarría, F., Font, J., Ruiz, S., García, E., Blanco, J.M., Jiménez-Gómez, F., Prieto, L., González-Alaminos, A., García, C.M., Cipollini, P., Snaith, H., Bartual, A., Reul and A., Rodríguez, V. (2001) Surface distribution of chlorophyll, particles and gelbstoff in the Atlantic jet of the Alborán Sea: from submesoscale to subinertial scales of variability. Journal of Marine Systems 29, 277-292.

Sarhan, T., García-Lafuente, J., Vargas, M., Vargas, J.M. and Plaza, F. (2000) Upwelling mechanisms in the northwestern Alboran Sea. Journal of Marine Systems. 23, 317-331.

Sbaffi, L., Wezel, F.C., Curzi, G. and Zoppi, U. (2004) Millennial- to centennial-scale palaeoclimatic variations during Termination I and the Holocene in the central Mediterranean Sea. Global Planetary Change 40, 201-217.

Scherer, R.P., Bohaty, S.M., Dunbar, R.B., Esper, O., Flores, J.A., Gersonde, R., Harwood, D.M., Roberts, A.P. and Taviani, M. (2008) Antarctic records of precession-paced insolation driven warming during early Pleistocene Marine Isotope Stage 31. Geophysical Research Letters 35, L03505.

Schlitzer, R. (2008) Ocean Data View. http://odv.awi.de.

Schulz, M. and Mudelsee, M. (2002) REDFIT: estimating red-noise spectra directly from 768 unevenly spaced paleoclimatic time series. Computers \& Geosciences 28, 421-426.

Siani, G., Paterne, M., Arnold, M., Bard, E., Métivier, B., Tisnerat, N. and Bassinot, F. (2000) Radiocarbon reservoir ages in the Mediterranean Sea and Black Sea. Radiocarbon 42, 271-280.

Sierro, F.J., Bárcena, M.Á., Flores, J.A., Cacho, I., Pelejero, C., Grimalt, J. and Shackleton, N. (1998) Origin of the youngest western Mediterranean organic-rich layer: productivity or stagnation. In: Abrantes, F. (Ed.), Reconstructing Ocean History, a Window Into the Future. 6th International Conference on Paleoceanography, Lisbon, p. 211.

Sierro, F.J., Hodell, D.A., Curtis, J.H., Flores, J.A., Reguera, I., Colmenero-Hidalgo, E., Bárcena, M.A., Grimalt, J.O., Cacho, I., Frigola, J.and Canals, M., (2005) Impact of iceberg melting on Mediterranean thermohaline circulation during Heinrich events. Paleoceanography 20, PA2019.

Stanford, J.D., Rohling, E.J., Bacon, S., Roberts, A.P., Grousset, F.E. and Bolshaw, M. (2011) A new concept for the paleoceanographic evolution of Heinrich event 1 in the North Atlantic. Quaternary Science Reviews 30, 1047-1066.

Stuiver, M. and Reimer, P.J. (1993) Extended 14C data base and revised CALIB 3.0 14C age calibration program. Radiocarbon 35, 215-230. 
Telford, R. (2012) palaeoSig: significance tests of quantitative palaeoenvironmental reconstructions. R Package Version, 1.1-1.

Telford, R.J. and Birks, H.J.B. (2011) A novel method for assessing the statistical significance of quantitative reconstructions inferred from biotic assemblages. Quaternary Science Reviews 30, 1272-1278.

Telford, R.J., Li, C. and Kucera, M. (2013) Mismatch between the depth habitat of planktonic foraminifera and the calibration depth of SST transfer functions may bias reconstructions. Climate of the Past 9, 859-870.

Thierstein, H.R. and Young, J.R. (Eds.). (2004) Coccolithophores: From Molecular Processes to Global Impact. Springer, Berlin (565 pp.).

Villanueva, J., Pelejero, C. and Grimalt, J.O. (1997) Clean-up procedures for the unbiased estimation of $\mathrm{C} 37$ alkenone sea surface temperatures and terrigenous n-alkane inputs in paleoceanography. Journal of Chromatography A 757, 145-151.

Weaver, P.P.E. and Pujol, C. (1988) History of the last deglaciation in the Alboran Sea (western Mediterranean) and adjacent north Atlantic as revealed by coccolith floras. Palaeogeography, Palaeoclimatology, Palaeoecology 64, 35-42.

Wiersma, A.P. and Renssen, H. (2006) Model-data comparison for the 8.2 ka BP event: confirmation of a forcing mechanism by catastrophic drainage of Laurentide Lakes. Quaternary Science Reviews 25, 63-88.

Winter, A. and Siesser, W.G. (Eds.). (1994) Coccolithophores. Cambridge Univ. Press, Cambridge, U. K. (242 pp.).

Zanchetta, G., Drysdale, R.N., Hellstrom, J.C., Fallick, A.E., Isola, I., Gagan, M.K. and Pareschi, M.T. (2007) Enhanced rainfall in theWestern Mediterranean during deposition of sapropel S1: stalagmite evidence fromCorchia cave (Central Italy). Quaternary Science Reviews 26, 279-286. 


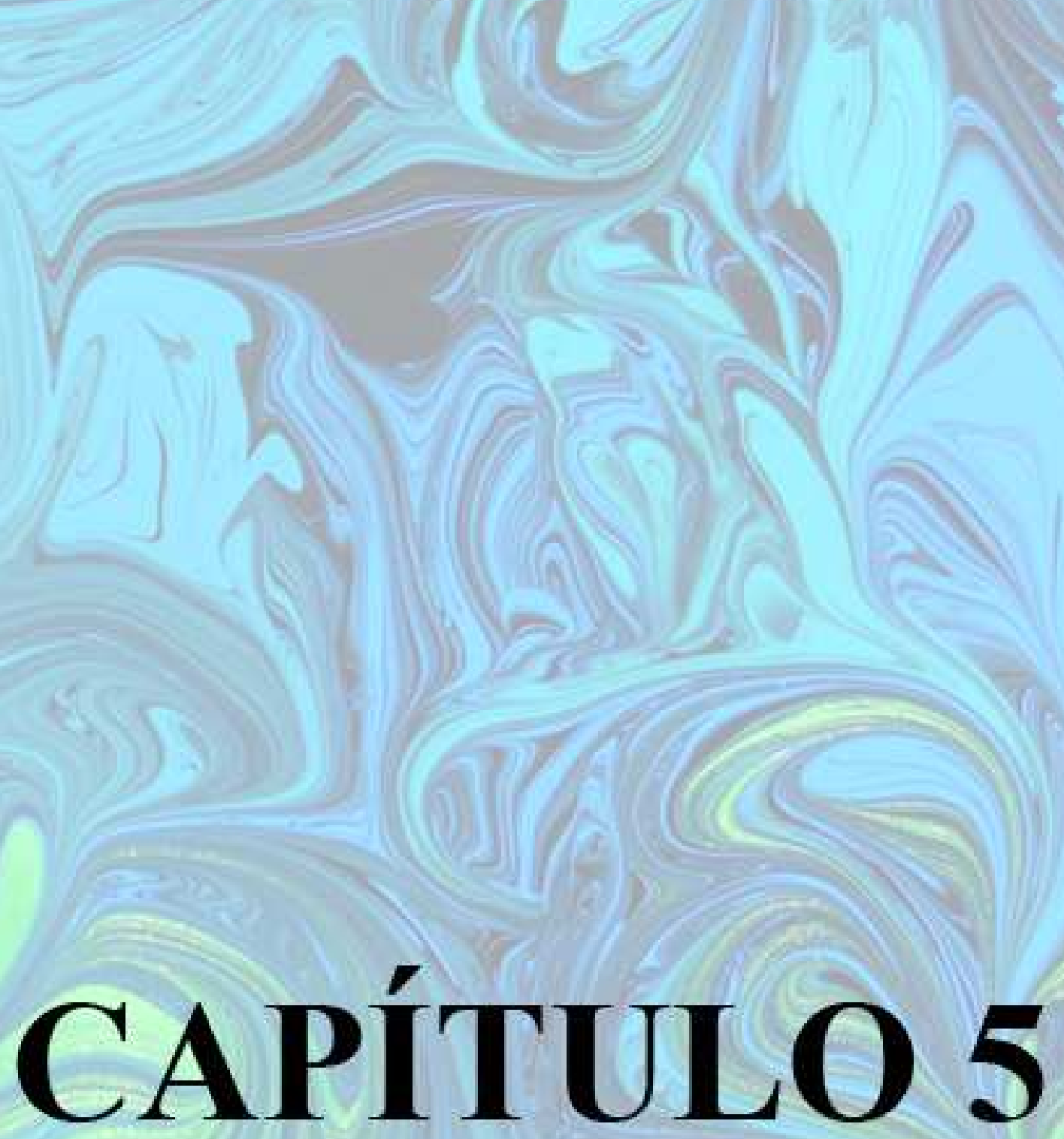





\section{5.-ATMOSPHERIC PATTERNS DRIVING HOLOCENE PRODUCTIVITY IN THE} ALBORAN SEA (WESTERN MEDITERRANEAN): A MULTIPROXY APPROACH

Blanca Ausín ${ }^{1}$; Jose A Flores ${ }^{1}$; Francisco J Sierro ${ }^{1}$; Isabel Cacho²; Iván Hernández-Almeida ${ }^{3}$; Belén Martrat ${ }^{4}$; Joan O Grimalt ${ }^{4}$

${ }^{1}$ Department of Geology, University of Salamanca. Pza/ de la Merced s/n, 37008 Salamanca, Spain.

${ }^{2}$ Department of Stratigraphy, Paleontology and Marine Geosciences, University of Barcelona, C/Martí i Franquès s/n, 08028 Barcelona, Spain.

${ }^{3}$ Institute of Geography and Oeschger Centre for Climate Change Research, University of Bern, Erlachstrasse 9a, CH-3012 Bern, Switzerland.

${ }^{4}$ Department of Environmental Chemistry. Institute of Environmental Assessment and Water Research, C/Jordi Girona 18, 08034 Barcelona, Spain.

Email: b_ausin@usal.es

Published in The Holocene (DOI: 10.1177/0959683614565952) 


\section{Resumen}

Las variaciones en la paleoproductividad en una célula productiva del mar de Alborán durante el Holoceno han sido reconstruidas a alta resolución. Para ello se han estudiado las asociaciones de cocolitóforos fósiles junto a la temperatura superficial marina derivada de alquenonas y otros indicadores paleoambientales. La aparición de esta célula se propone en 7,7 ka cal. BP y ha sido ligada al establecimiento del giro anticiclónico occidental. A partir de 7,7 ka hasta el presente, la tasa de acumulación de Florisphaera profunda revela episodios sucesivos de debilitamiento y refuerzo de las condiciones de afloramiento en el mar de Alborán, simultáneos a cambios en la formación de agua mediterránea profunda occidental (WMDW) ${ }^{4}$ en el golfo de León. Se ha propuesto un escenario con dos fases alternantes para explicar las variabilidades climática y oceanográfica encontradas a escala milenaria y secular: (1) contemporáneos con condiciones climáticas más áridas, vientos Mistral y Tramontana relativamente débiles habrían desencadenado una reducción en la formación de WMDW. Este proceso, junto a un menor influjo atlántico $(\mathrm{AJ})$ en el mar de Alborán habría dado lugar a una menor mezcla vertical y, por tanto, a una columna de agua más estable en el área de estudio y una menor productividad; (2) condiciones climáticas más húmedas habrían prevalecido en la región mientras vientos Mistral y Tramontana relativamente fuertes habrían promovido un refuerzo en la producción de WMDW en el golfo de León simultáneo con una intensificación del AJ que en consecuencia, habría migrado hacia el sur. Esto habría incrementado la mezcla vertical, intensificando las condiciones de afloramiento en el área de estudio. Aquí, el patrón invernal de la Oscilación del Atlántico Norte (NAO) es considerado como un mecanismo de forzamiento relevante para la variabilidad encontrada, influenciando la formación de WMDW, que a su vez ha sido ligada a las variaciones a corto plazo de productividad durante los últimos 7,7 ka en el mar de Alborán.

Palabras clave: mar de Alborán, productividad de cocolitóforos, Holoceno, Oscilación del Atlántico Norte, Agua Mediterránea Profunda Occidental.

\footnotetext{
Abstract

${ }^{4}$ Los acrónimos siguen la nomenclatura anglosajona por razones de consistencia con el resto del texto.
} 
High-resolution paleoproductivity variations have been reconstructed in a productive cell in the Alboran Sea for the Holocene. Fossil coccolithophore assemblages have been studied along with the $\mathrm{U}^{\mathrm{k}^{\prime}}{ }_{37}$-estimated sea-surface temperature (SST) and other paleoenvironmental proxies. The appearance of this cell is suggested at $7.7 \mathrm{ka}$ cal BP and was linked to the establishment of the western anticyclonic gyre. From that time until the present, the nannofossil accumulation rate of Florisphaera profunda has revealed successive episodes of weakening and strengthening of upwelling conditions in the Alboran Sea that have been simultaneous to changes in Western Mediterranean Deep Water (WMDW) formation in the Gulf of Lions. A two-phase scenario operating at millennial-centennial time-scale is proposed to explain this climatic and oceanographic variability: [1] coeval with more arid climate conditions, weaker northerlies/north-westerlies blowing over the Gulf of Lions would have triggered a slackening of WMDW formation. This together with a minor Atlantic Jet (AJ) inflowing into the Alboran Sea would have led to less vertical mixing, and hence, a more stable water column in the study area; [2] wetter climate conditions would have prevailed in the region while stronger northerlies/north-westerlies would have enabled WMDW reinforcement in the Gulf of Lions simultaneous to an intensification of the AJ that migrated southward. This would have increased vertical mixing, intensifying upwelling conditions in the study area. Here, the winter North Atlantic Oscillation (NAO) is considered to be an important forcing mechanism for this variability, influencing WMDW formation, which in turn has been linked to short-term productivity variations during the last $7.7 \mathrm{kyr}$ in the Alboran Sea.

Keywords: coccolithophore productivity, Holocene, Western Mediterranean Deep Water, North Atlantic Oscillation, Alboran Sea.

\section{1.-INTRODUCTION}


In recent years, climatic variability during the Holocene (11.7 kyr to present) has been a challenging issue. Recent studies have shown that this period has been characterised by several abrupt climatic events as well as pervasive short-term oscillations (Mayewski et al., 2004; Wanner et al., 2011), ruling out the perception of the Holocene as having been stable. As the most recent geological period, the variability recorded by the natural system during this time must be taken into account when attempting to project future climatic scenarios (IPCC, 2013). Identifying the causes of this variability, as well as the mechanisms transferring it from one region to another, is crucial if we are to gain an overall understanding of the system.

The Western Mediterranean is an essential region as regards determining climatic teleconnections with the North Atlantic area (Cacho et al., 1999; Martrat et al., 2004; Sierro et al., 2005). Current sea-level, temperature and precipitation variability in the Western Mediterranean have been linked to the fluctuation of the atmospheric gradient formed by the Azores high- and the Icelandic low- pressure centers: the North Atlantic Oscillation (NAO) (Tsimplis y Josey, 2001; Trigo et al., 2006), a natural mode of atmospheric variability that has a pronounced effect on the climate of western central Europe at decadal time scales (Hurrell, 1995). The intensity of a NAO-like pattern has already been suggested as a likely driving mechanism for several short-term environmental oscillations in the Western Mediterranean during the Holocene (Jalut et al., 1997; Jalut et al., 2000; Goy et al., 2003; Frigola et al., 2007; Fletcher y Zielhofer, 2011; Fletcher et al., 2012). However, little evidence is available concerning the impact of short-term climatic oscillations on past ocean productivity in the Mediterranean Sea (Abrantes et al., 2012) or regarding its probable connection with North Atlantic climatic processes. Within the generalized oligotrophic character of the Mediterranean Sea, the Alboran Sea is considered an exception, exhibiting quasi-permanent areas of upwelling (Sarhan et al., 2000) where local vertical mixing is the main factor controlling marine productivity (Dafner et al., 2003). Upwelling dynamics are steered by local hydrography and atmospheric circulation (García-Gorriz y Carr, 1999). The pattern is as follows: offshore upwelling associated with southward drifting of the Atlantic Jet (AJ), and wind-induced coastal upwelling on the shore, promoted by winds blowing along the Spanish coast (Sarhan et al., 2000). The semi-enclosed features of the Alboran Sea lead to partial isolation of these phenomena, making it an ideal region for the study of the impact of short-term climatic oscillations on ocean productivity. Its latitudinal position and its connection with the Atlantic Ocean also provide a reasonable area for the study of ocean-climate teleconnections between northern processes and lower latitudes.

Coccolithophores are Haptophyte algae with calcified scales (coccoliths). Living forms are one of the major oceanic primary producers, and they are strongly influenced by nutrient 
availability, dissolved $\mathrm{CO}_{2}$ concentrations in sea water, and SST, among others (Thierstein y Young, 2004). Thus, fossil forms of coccoliths preserved in deep-sea sediments are commonly used as a widespread proxy to reconstruct some of these variables as well as productivity (Giraudeau, 1992; Flores et al., 1999; Flores et al., 2000; Baumann y Freitag, 2004; ColmeneroHidalgo et al., 2004).

The main objective of the present study was to reconstruct past productivity in an upwelling area of the Alboran Sea during the Holocene and to elucidate the climatic and oceanographic mechanisms involved in past variations in productivity, especially those related to North Atlantic climatic and oceanographic dynamics. For this reason, we report here a marine productivity record reconstructed from fossil coccolithophores along with data on oxygen isotopes, alkenone-estimated SST and other organic biomarkers for the last $12 \mathrm{kyr}$ and correlate them with data referring to paleoenvironmental variations.

\section{2.-AREA OF STUDY: MODERN WATER MASSES AND CLIMATIC DYNAMICS}

Core HER-GC-T1 was recovered off the coast of Malaga (Fig. 5.1b) in the Alboran Sea (Western Mediterranean). This is a transitional region where Atlantic Water (AW) enters the Mediterranean Sea through the Strait of Gibraltar as a jet of water called the Atlantic Jet (AJ) (García-Lafuente et al., 2000), and becomes two quasi-permanent anticyclonic gyres on its way to the east: the Western Anticyclonic Gyre (WAG) and the eastern one (EAG) (Heburn y La Violette, 1990). The interaction between AW and the more saline and warmer Mediterranean water (MW) results in the formation of a geostrophic front at the northern limit of the WAG, called the Alboran Front (where HER-GC-T1 is located) (Minas et al., 1991) (Fig. 5.1b). Southward migrations of the AJ allow the water from below, relatively warm and fresh, to upwell in the study area (Sarhan et al., 2000) forming a high-productivity cell referred to here as the "Malaga cell".

At depth, water circulation may be simplified with a three-layer model: on the surface, the mixing of AW and MW forms the Modified Atlantic Water (MAW), occupying the photic zone (100-200 m). Below, Levantine Intermediate Water (LIW), formed in the eastern part of the Mediterranean Sea, flows at a depth of 200-600 m towards the Strait of Gibraltar. Below the LIW, Western Mediterranean Deep Water (WMDW) flows at 600-3000 $\mathrm{m}$ depth in the same direction (Millot, 1999). WMDW is formed in the open sea off the Gulf of Lions (Fig. 5.1c) (MEDOCGROUP, 1970). This deep water convection has a thermohaline origin and is linked to buoyancy preconditions determined by the heat flux, which in turn is steered by the blowing winds: the Tramontana (northerlies) and/or Mistral (north-westerlies) (Mertens y Schott, 1998; Rixen et al., 2005; Font et al., 2007; Smith et al., 2008). These cold dry winds blowing over the 
area induce heat losses and the evaporation of the MAW, which becomes saltier and colder and finally sinks owing to its high density, to form the WMDW (Font et al., 2007).
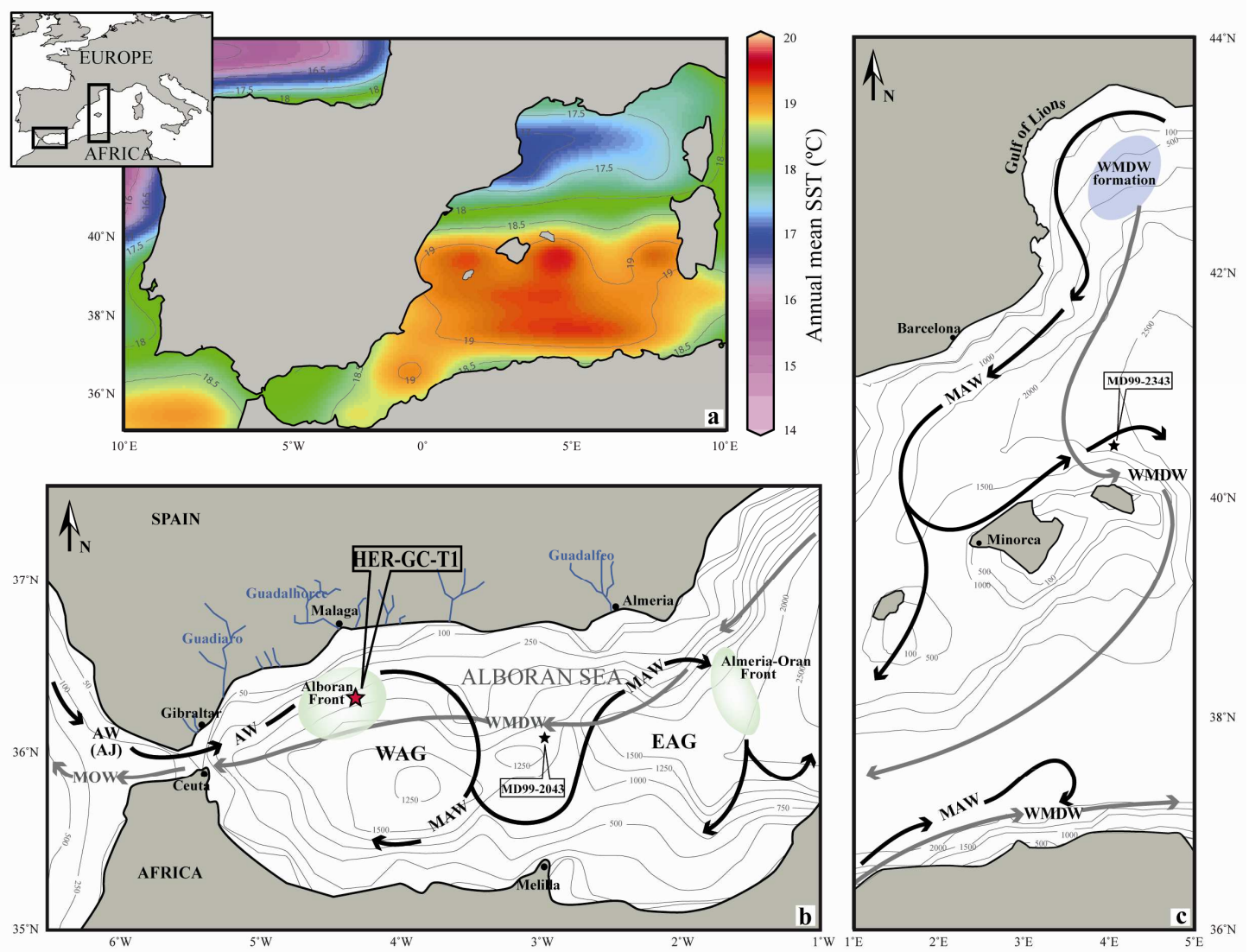

Figure 5.1. Study area. a) Map of the annual mean SST $\left({ }^{\circ} \mathrm{C}\right)$ in the Western Mediterranean Sea plotted with Ocean Data View (Brown, 1998). b) Study area and HER-GC-T1 core location in the Alboran Sea. c) Gulf of Lions area where WMDW formation occurs. Black arrows represent general superficial circulation. Grey arrows trace general deep circulation. AW: Atlantic Water, entering the Alboran Sea as the Atlantic Jet: AJ. MAW: Modified Atlantic Water. WMDW: Western Mediterranean Deep Water. MOW: Mediterranean Outflowing Water. WAG: Western Anticyclonic Gyre. EAG: Eastern Anticyclonic Gyre.

Present climate conditions in the region are influenced by an atmospheric high-pressure center above the Azores archipelago in the Atlantic Ocean resulting in hot dry summers and wetter winters (Sumner et al., 2001). At decadal and inter-annual time-scales, climatic variability in the North Atlantic region is modulated by the NAO (Hurrell, 1995). Currently, winter anomalies of the NAO in the Mediterranean have been shown to influence sea level variability (Tsimplis y Josey, 2001), wave climate (Cañellas et al., 2010) and temperature and precipitation trends to a significant extent (Trigo et al., 2006). The NAO has been suggested to exert an indirect influence on net water flux in the Strait of Gibraltar via its high correlation with regional evaporation, precipitation and runoff (Fenoglio-Marc et al., 2013). The winter- 
NAO has also been correlated with heat flux anomalies that determine the buoyancy preconditions of deep water convection in the Gulf of Lions (Rixen et al., 2005), although Josey et al. (2011), using a broader definition of winter (October-March), concluded that the NAO only plays a secondary role when other modes of variability related to the heat flux are considered.

\section{3.-MATERIALS AND METHODS}

We analyzed the top $183 \mathrm{~cm}$ of gravity core HER-GC-T1 (Lat. $36^{\circ} 22^{\prime} 12^{\prime \prime} \mathrm{N}$, Long. $\left.4^{\circ} 17^{\prime} 57^{\prime \prime} \mathrm{W}\right)$, recovered by the BIO Hespérides during the Hermesione research cruise in 2009 , from a depth of 658.9 meters below sea level (mbsl). The sediments recovered are mainly composed of dark greenish-gray mud rich in planktonic foraminifers.

\subsection{1.-Age model}

Table 5.1

Age model for core HER-GC-T1. ${ }^{\mathrm{a} P o z n a n}$ Radiocarbon Laboratory. ${ }^{\mathrm{b}}$ Woods Hole Oceanographic Institution.

\begin{tabular}{|c|c|c|c|c|}
\hline $\begin{array}{c}\text { Radiocarbon } \\
\text { (Sample/Laboratory code) }\end{array}$ & Foram Type & $\begin{array}{l}\text { Depth } \\
\text { (cm) }\end{array}$ & $\begin{array}{c}\text { Radiocarbon } \\
\text { Age } \\
(y r B P)\end{array}$ & $\begin{array}{c}\text { Calendar Age (2- } \\
\text { sigma error } \\
\text { range }) \\
\text { (yr cal. BP }) \\
\end{array}$ \\
\hline SEC1_2/ Poz-53233 ${ }^{\mathrm{a}}$ & G. inflata & 2 & $440 \pm 25$ & $88 \pm 62$ \\
\hline SEC1_21/OS-87586 ${ }^{\mathrm{b}}$ & G. inflata & 21 & $1,810 \pm 25$ & $1,379 \pm 54$ \\
\hline SEC1_63/ Poz-53234 & G. inflata & 63 & $4,175 \pm 35$ & $4,284 \pm 76$ \\
\hline SEC2_17/ Poz-53235 & G. inflata & 107 & $6,100 \pm 40$ & $6,550 \pm 68$ \\
\hline SEC2_54/OS-87587 & $\begin{array}{l}\text { G. inflata and } \\
N . \text { pachyderma } \\
\text { (dex) }\end{array}$ & 144 & $7,350 \pm 35$ & $7,834 \pm 58$ \\
\hline SEC3_12/ Poz-53236 & $\begin{array}{c}\text { N. pachyderma } \\
\text { (dex) }\end{array}$ & 202 & $10,400 \pm 60$ & $11,539 \pm 162$ \\
\hline
\end{tabular}

Radiocarbon ages were determined on six samples of selected foraminiferal shells using the accelerator mass spectrometry (AMS) technique (Table 5.1) at the Poznan Radiocarbon Laboratory and Woods Hole Oceanographic Institution. Conversion from radiocarbon ages to calibrated calendar years was performed using the OxCal 4.2 online software (Bronk Ramsey, 2008) and the Marine13 calibration dataset curve (Reimer et al., 2013), which includes a correction of $400 \mathrm{yr}$ for the global marine reservoir effect. The regional difference from this global reservoir correction $(\Delta \mathrm{R})$ (Stuiver y Braziunas, 1993) proved to be $-22 \pm 35$ years (Siani 
et al., 2000) and was also considered. The age model for the last $12 \mathrm{kyr}$ was based on linear interpolation between these six calendar ages (Fig. 5.2) performed with the AnalySeries Version 1.1 (Paillard et al., 1996). The average sedimentation rate was found to be $18.41 \pm 5.4 \mathrm{~cm}^{*} \mathrm{kyr}^{-1}$. All dates reported in the text are given in calendar ages BP.

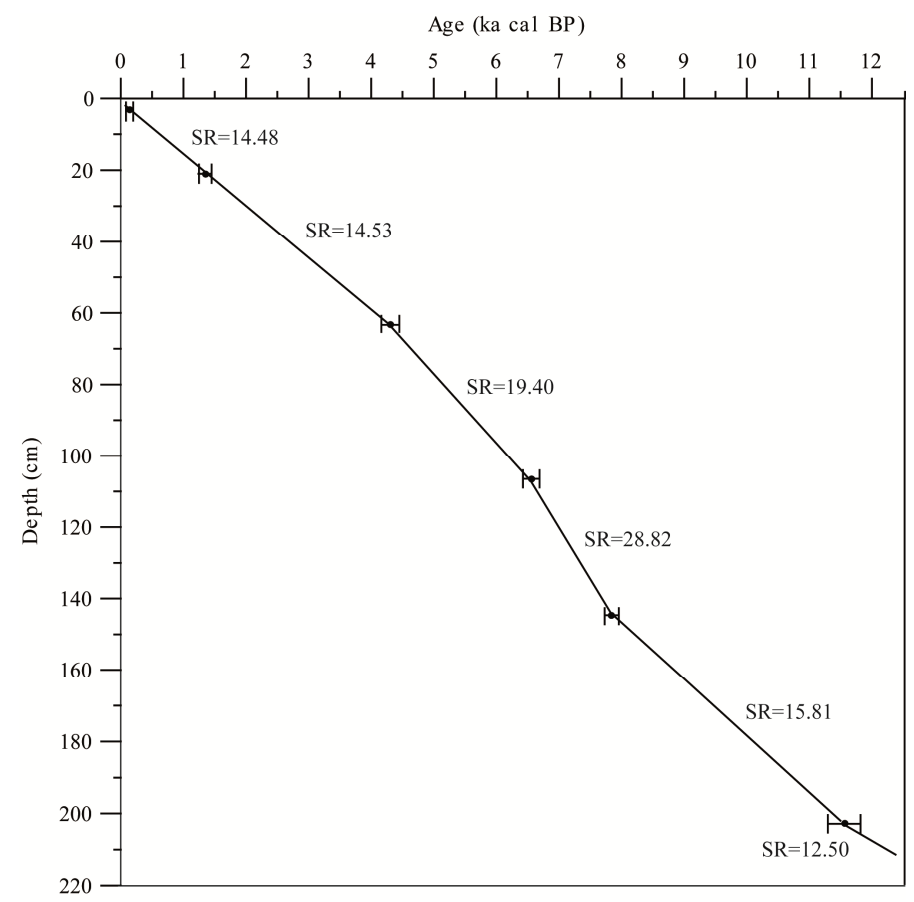

Figure 5.2. Age-depth model. Age control points are marked by a black dot and associated error bars. The solid line joining them is the age given in ka cal. BP. SR stands for sedimentation rate, given in $\mathrm{cm}^{*} \mathrm{kyr}^{-1}$.

\subsection{2.-Coccolithophore analysis and taxonomy}

Eighty-five samples were taken systematically every $2-3 \mathrm{~cm}$. The resulting sampling gives an average time resolution of $\sim 140$ years. Slides for micropaleontological analyses were prepared following the settling technique proposed by Flores and Sierro (1997). Qualitative and quantitative analyses were performed using a Nikon Eclipse 80-i petrographic microscope with a phase contrast device at 1000x magnification. Nannofossil census counts were based on at least 500 specimens identified in a first count, which is representative for studying the main species (Fatela y Taborda, 2002). In a second count, 25 fields of view were observed in order to avoid underestimation and/or overestimation of the taxa whose abundance was less than $1 \%$ in the first count. The Nannofossil Accumulation Rate (NAR) is given in numbers of coccoliths $* \mathrm{~cm}^{-2} * \mathrm{kyr}^{-1}$, and was calculated in each sample by considering dry-sediment density, the sedimentation rate and the absolute abundance of each species (number of coccoliths $\mathrm{g}^{-1}$ ). The total NAR was interpreted as a proxy of primary productivity. Relative abundance (\%) was also calculated. All taxa identified in this study have been reported previously for the sampling 
location (Weaver y Pujol, 1988; Álvarez et al., 2010). The "small Gephyrocapsa" group is integrated by all Gephyrocapsa specimens smaller than $3 \mu \mathrm{m}$ (Flores et al., 1999). Along with Emiliania huxleyi $(<4 \mu \mathrm{m})$, they were lumped together as "small placoliths". Other taxa identified in this study were Gephyrocapsa muellerae, Gephyrocapsa oceanica, Helicosphaera carteri, Syracosphaera spp. and Florisphaera profunda (as dominant taxa). The rare taxa identified were Braarudosphaera bigelowii, Calcidiscus leptoporus, Calciosolenia murrayi, Coccolithus pelagicus subsp. braarudii, Coccolithus pelagicus subsp. pelagicus, Gephyrocapsa caribbeanica, Oolithotus fragilis, Pontosphaera spp., Rhabdosphaera clavigera, Umbilicosphaera sibogae, Umbellosphaera spp. and Discosphaera tubifera. The latter four were lumped together as the warm-water group (WWG) owing to their common and relatively high record in warm waters (McIntyre y Bé, 1967; Okada y Honjo, 1973). Taxa pertaining to older stratigraphic levels (regularly older than the Pliocene in this study) were deposited again after their resuspension and transport to the core location and were also counted as rare species and designated "reworked specimens".

The preservation of the coccolithophore assemblages is good (little or no evidence of dissolution; diagnostic features fully preserved) (Flores y Marino, 2002). Moreover, the NARs were transformed into coccoliths $* \mathrm{~m}^{-2 *} \mathrm{day}^{-1}$. The results are comparable to the annual flux found by Bárcena et al. (2004) in the same area for sediment-trap samples, showing that dissolution and taphonomic effects are negligible in this core.

\subsection{3.-Oxygen stable isotopes}

Up to 20 well-preserved specimens of the planktic foraminifer Globigerina bulloides were picked from the $>200 \mu \mathrm{m}$ size fraction in 66 samples. The individuals were crushed, subjected to ultrasound and cleaned with methanol before isotopic analyses were performed with a SIRA mass spectrometer equipped with a VG isocarb common acid bath system at the University of Barcelona. Calibration to the Vienna Pee Dee Belemnite (VPDB) standard scale (Coplen, 1996) was accomplished using the NBS-19 standard, and analytical precision was better than $0.06 \%$ for $\delta^{18} \mathrm{O}$.

\subsection{4.-Molecular biomarkers}

A total of 86 samples, taken every 2-3 cm, were selected for the analysis of fossil organic compounds (long chain alkenones, alcohols and hydrocarbons). The experimental procedures used are described in Villanueva et al. (1997). Samples were analysed with a Varian Gas Chromatograph (GC) model 450, an autoSampler 8400, a Cold On-Column (COC) Injector 1093 and a Flame Ionization Detector (FID). The carrier gas was hydrogen $(2.5 \mathrm{~mL} / \mathrm{min}) . \mathrm{C}_{37}$ unsaturated alkenones (di-unsaturated and tri-unsaturated) are synthesized by coccolithophorid 
flora. Their identification and quantification allow the calculation of the $\mathrm{U}^{\mathrm{k}^{\prime}}{ }_{37}$ index, which was calibrated according to the equation proposed by Müller et al. (1998) in order to measure SST. The total concentration of $\mathrm{C}_{37}$ alkenones $\left(\left[\mathrm{C}_{37: 2}+\mathrm{C}_{37: 3}\right]\right)$ was also calculated and is interpreted as a proxy for poor/good preservation of organic matter in deep sea sediments in relation to well/poorly ventilated deep waters (Cacho et al., 2002). The $n$-hexacosan-1-ol index was calculated through the relative ratio of $n$-hexacosan-1-ol $\left(\mathrm{C}_{26} \mathrm{OH}\right)$ to the sum of $\left(\mathrm{C}_{26} \mathrm{OH}\right)$ plus $n$ nonacosane $\left(\mathrm{C}_{29}\right)$. Since $n$-hexacosanol is more labile to degradation processes than $n$ nonacosane, decreases in the $n$-hexacosan-1-ol index can be interpreted as a higher ventilation of the deep basin (Cacho et al., 2000).

\subsection{5.-Statistical approaches}

In order to search for possible relationships between some of the proxies addressed here, statistical cross-correlation, which is suitable for temporal series, was carried out using PAST 3.01 software (Hammer, 2001). This method requires evenly sampled temporal data, which were interpolated regularly every 0.14 kyr (the lowest resolution among those from all of the records correlated).

\section{4.-RESULTS}

\subsection{1.-Calcareous nannoflora distribution}

On average, small placoliths constitute up to $80 \%$ of the nannofossil assemblage (Fig. 5.3). Their NAR records maximum values from 7.7 to $6.2 \mathrm{ka}$. G. muellerae (Fig. 5.3) is the only species that records its highest NAR from 12 to $10.5 \mathrm{ka}$, followed by a decreasing trend from $10.5 \mathrm{ka}$ onwards, interrupted only by higher values from 7.7 to $6.2 \mathrm{ka}$. The NAR of the WWG is scarce for the whole period studied, except from 7.7 to $6.4 \mathrm{ka}$ when it records higher values (Fig. 5.3). F. profunda and small G. oceanica exhibit similar general variability (Fig. 5.3): a low NAR up to $7.7 \mathrm{ka}$, when a large peak is observed; from then onwards up-core, they record higher NAR values as well as large oscillations.

Reworked specimens are mainly constituted by Upper Cretaceous, Paleogene and Neogene specimens. On average the percent of this allochthonous group relative to autochthonous taxa (Fig. 5.3) is $0.7 \%$ for the whole record. However, it shows five peaks up to $1.6 \%$ at $7.0,6.2$, 5.0, 4.1 and $2.8 \mathrm{ka}$.

The total NAR (Fig. 5.3) shows an increasing trend from 12.5 to 7.7 ka. From that time onwards up to $6.5 \mathrm{ka}$, it records its highest values followed by a decreasing trend up-core. 


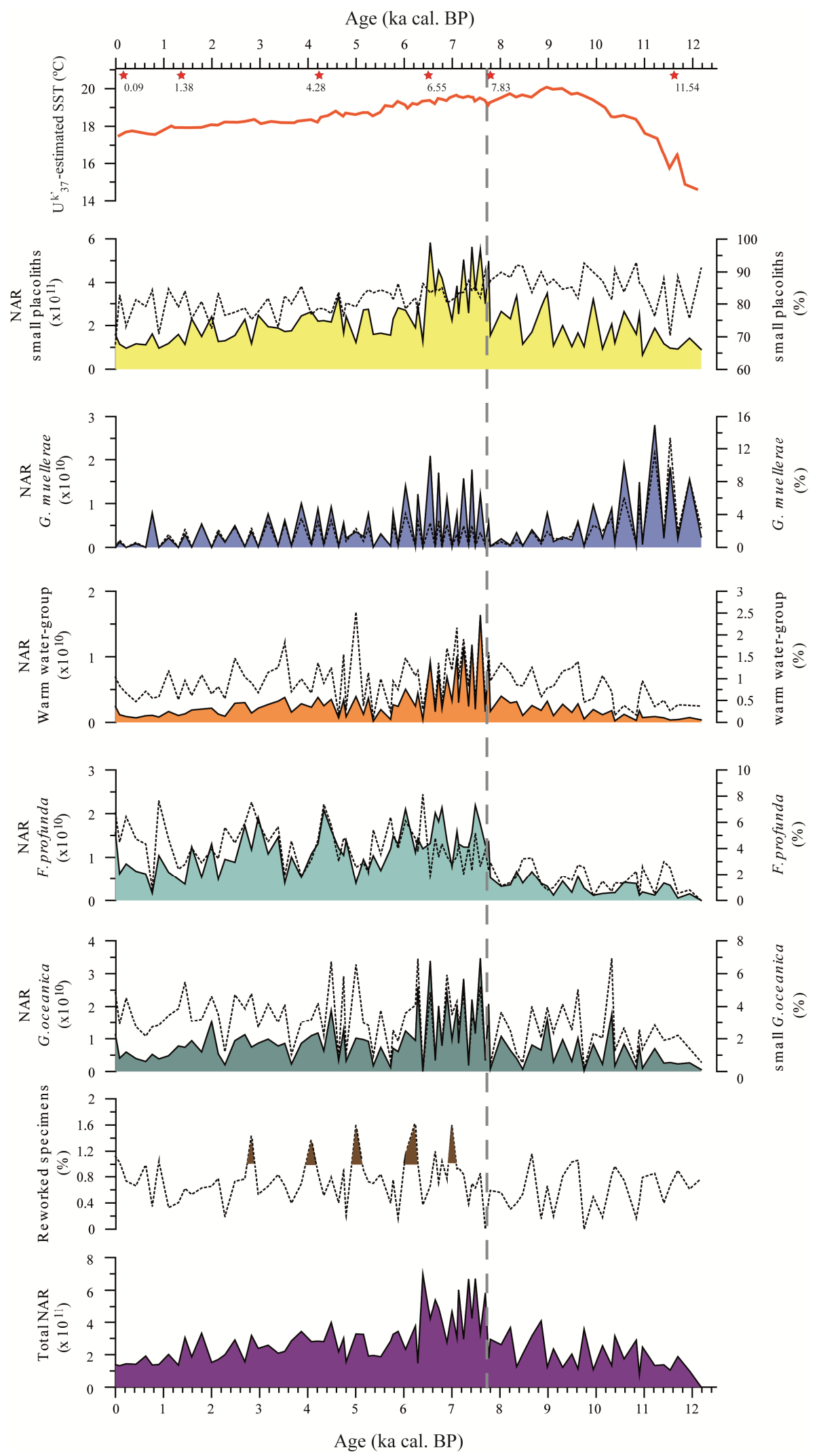

Figure 5.3. $\mathrm{U}^{\mathrm{k}^{\prime}}{ }_{37}$-estimated $\mathrm{SST}\left({ }^{\circ} \mathrm{C}\right)$. Nannofossil Accumulation Rate (NAR) (black lines) and relative abundance (\%) (dashed line) of the coccolithophore assemblage. The grey dashed vertical line indicates the $7.7 \mathrm{ka} \mathrm{cal}$. BP event. Red stars represent age control points (ka cal. BP) (Table 5.1). 


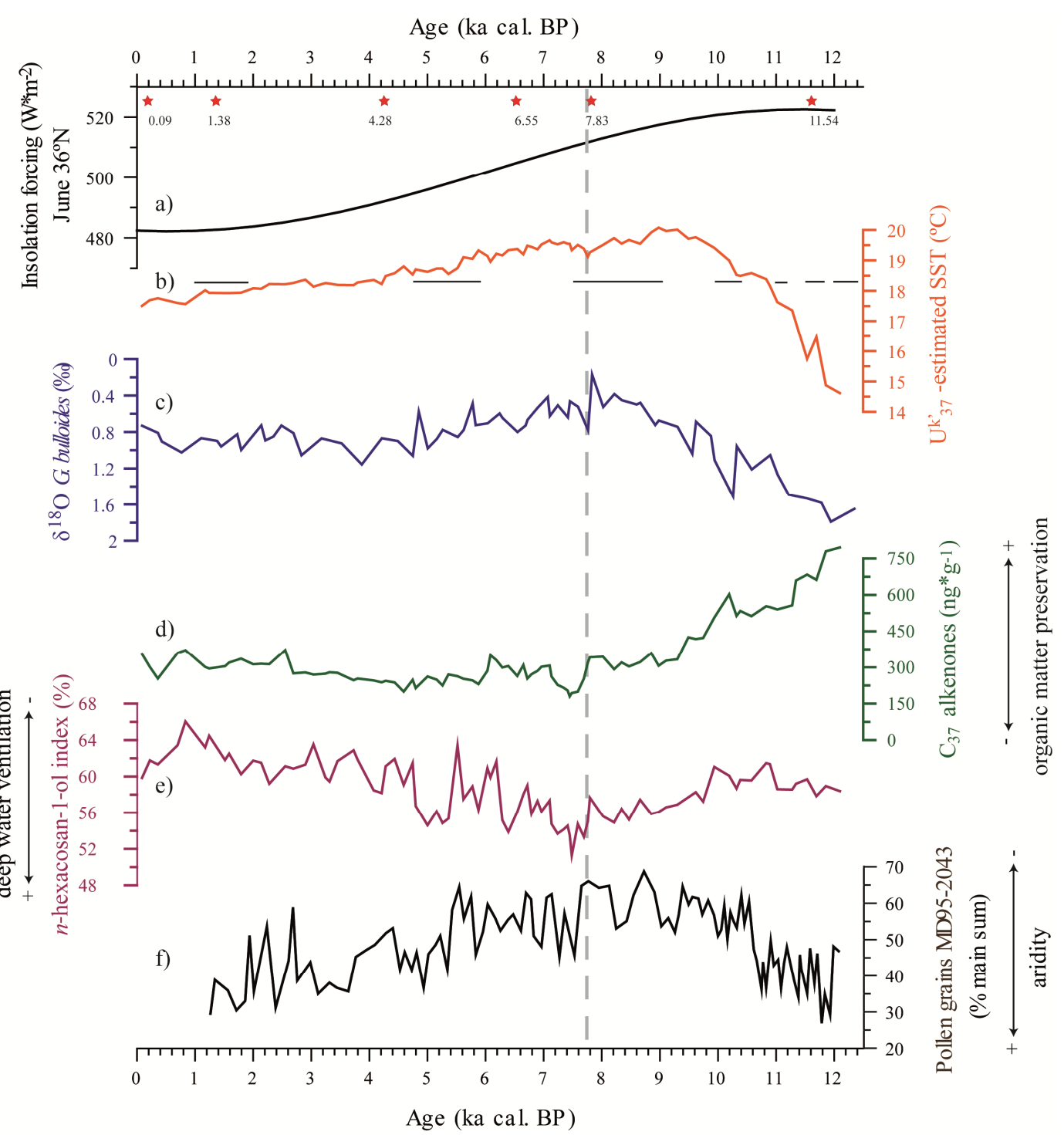

Figure 5.4. a) Insolation curve (June, $36^{\circ} \mathrm{N}$ ) (Berger, 1978). b) $\mathrm{U}^{\mathrm{k}^{\prime}}{ }_{37}$-estimated SST $\left({ }^{\circ} \mathrm{C}\right)$. Horizontal lines mark the rapid Holocene cooling events identified by Cacho et al. (2001). c) $\delta^{18} \mathrm{O}$ record (\%) (Note that the vertical axis is reversed). d) Concentration of $\mathrm{C}_{37}$ alkenones $\left(\left[\mathrm{C}_{37: 2}+\mathrm{C}_{37: 3}\right]\right)\left(\mathrm{ng}^{*} \mathrm{~g}^{-1}\right)$. e) $n$ hexacosan-1-ol index (\%). f) Pollen grains from core MD95-2043 in the Alboran Sea for all temperate and Mediterranean forest taxa (Fletcher et al., 2012).

\subsection{2.-Sea-Surface Temperature}

$\mathrm{U}^{\mathrm{k}^{\prime}}{ }_{37^{-}} \mathrm{SST}$ (Fig. 5.3) has its absolute minimum $\left(14.6^{\circ} \mathrm{C}\right)$ at $12 \mathrm{ka}$, and its absolute maximum $\left(20.1^{\circ} \mathrm{C}\right)$ at $8.9 \mathrm{ka}$. A decreasing trend is observed from $8.9 \mathrm{ka}$ up-core, characterized by a generalized smooth internal variability. More specifically, short cooling events (up to $1^{\circ} \mathrm{C}$ ) are seen centered at $11.5,10.4,8.5,7.7,5.3$, and $0.8 \mathrm{ka}$. 


\subsection{3.-Oxygen isotopic record}

As from $12 \mathrm{ka}$ (Fig. 5.4c) this record tends to lower values, its absolute minimum being reached at $7.8 \mathrm{ka}$ and its values varying up to $1.48 \%$ during that period. From 7.8 to $3.8 \mathrm{ka}$ several slight depletions interrupt a trend to higher values, increasing by a total of $0.98 \%$. From $3.8 \mathrm{ka}$ to the top, $\delta^{18} \mathrm{O}$ tends towards slightly lower values, with a range of $0.43 \%$.

\subsection{4.-Total concentration of $C_{37}$ alkenones and the $n$-hexacosan-1-ol index}

The total concentration of $\mathrm{C}_{37}$ alkenones (Fig. 5.4d) shows higher values from 12 to $9 \mathrm{ka}$. This record is affected by a decreasing trend up to $7.5 \mathrm{ka}$, and from that time onwards up-core it shows low values and little variability. The $n$-hexacosan-1-ol index (Fig. 5.4e) tends towards lower values from 12 to $7.5 \mathrm{ka}$. After the absolute minimum recorded at $7.5 \mathrm{ka}$, a trend towards higher values is recorded up-core, punctuated by several depletions.

\section{5.-DISCUSSION}

\subsection{1.-General primary productivity and SST trends}

Holocene productivity can be divided into three periods according to the total NAR variability (Fig. 5.3): from 25.5 to $7.7 \mathrm{ka}$, characterised by low productivity and a slight increasing trend; from 7.7 to $6.2 \mathrm{ka}$, when productivity reached its highest values; and from 6.2 ka onwards, a period affected by lower productivity and a slight decreasing trend up-core.

Specifically from 12 to $10.5 \mathrm{ka}$, low SST values correspond to a high G. muellerae NAR and relative abundances and a low WWG NAR and relative abundances (Fig. 5.3), customarily used as cold- and warm-water proxies, respectively (McIntyre y Bé, 1967; Okada y Honjo, 1973; Weaver y Pujol, 1988).

The long-term cooling trend shown by the $\mathrm{U}^{\mathrm{k}^{\prime}}{ }_{37}$-SSTs from 9 ka during the Holocene is in agreement with the findings of Marchal et al. (2002) from the study of seven cores from the northeast Atlantic and the Mediterranean Sea. However, from 7.7 to 6.2 ka there is no agreement between the total NAR and the SST cooling trend, suggesting that factors other than SST would have controlled coccolithophore production and variability during that time. Specifically, the signal of WWG (Fig. 5.3) results from the sum of all taxa with a preference for warm waters. This does not exclude the possibility that other environmental parameters such us salinity, nutrients, eddies and species-specific biogeography could affect their distribution and variability (Baumann et al., 2005) and hence their signal in palaeorecords.

Despite this general cooling trend, the G. muellerae NAR and relative abundances decrease from $6.2 \mathrm{ka}$ up-core, showing similar variability to that of the small placoliths, which are eurythermal species linked to the presence of nutrients (Okada and Wells, 1997). Both taxa 
could have been controlled by the same factors since they show similar responses. Flores et al. (1997) found low abundances of G. muellerae after the early part of the last Pleniglacial (73.9 kyr) in the Western Mediterranean, suggesting that the affinity of this species for cold-water conditions is unclear. We suggest that the abundance of G. muellerae has been responding not only to colder waters but also to nutrient availability as from $7.7 \mathrm{ka}$.

The NAR and relative abundances of $F$. profunda (Fig. 5.3) increase at $7.7 \mathrm{ka}$ and undergo several high-amplitude oscillations up-core as from that time. This species inhabits the lower photic zone (LPZ) and is abundant under a deep nutricline and an upper photic zone (UPZ) impoverished in nutrients (Molfino y McIntyre, 1990), a characteristic that affected the water column in the Alboran Sea during several periods of the last $7.7 \mathrm{ka}$.

The general trends shown by the SST profile are similar to that shown by the $\delta^{18} \mathrm{O}$ record (Fig. 5.4). From 8.9 ka both profiles follow a decreasing trend in agreement with that of the insolation curve (Fig. 5.4a) (Berger, 1978). This suggests that insolation could have played an important role from that time onwards along the Holocene as a long-term factor influencing the SST, which in turn would have had an important effect on the isotopic composition of oxygen recorded in foraminiferal shells.

With regard to deep basin conditions, a period of well-preserved organic matter from 12 to $9.5 \mathrm{ka}$ can be deduced from the higher concentration of $\mathrm{C}_{37}$ alkenones (Fig. 5.4d). This period coincides with the last part of the Organic-Rich Layer (ORL-1) reported by Cacho et al. (2002) in the Alboran Sea due to an oxygen-depleted environment during the deglaciation. The preservation of organic matter declines until $7.5 \mathrm{ka}$, coherent with the gradual increase in deep water ventilation shown by the $n$-hexacosan-1-ol index (Fig. 5.4e). From these conditions, we deduce a period of thermohaline reactivation that gradually decreases from 7.5 ka onwards. During this latter period, the preservation of the organic matter is relatively low, although a slight increasing trend up-core can be recognized in the total concentration of $\mathrm{C}_{37}$ alkenones, in agreement with the gradual reduction in deep water ventilation (Fig. 5.4e), while, according to the total NAR, lower productivity affects the photic zone (Fig. 5.3).

According to Fletcher et al. (2012), continental aridity is evidenced by polen analysis of the marine core MD95-2043 (Figs. 5.1b and 5.4f). This record shows a decreasing abundance of Mediterranean and temperate pollen grains up-core, pointing to a gradual decline in forest mass and more arid conditions during the Holocene. 


\subsection{2.-Short-term changes}

\subsubsection{1.-The 7.7 ka event}

At $7.7 \mathrm{ka}$ there is a simultaneous increase in the NAR of all dominant taxa (Fig. 5.3). The high sedimentation rate from 7.7 to $6.2 \mathrm{ka}$ (Fig. 5.2) is partly responsible for this increase in NARs, since these depend on the former for its calculation. Nevertheless, the absolute abundances (coccoliths $\mathrm{g}^{-1}$ ) of these taxa also reflect this sharp increase at that time (data not shown), from which it may be deduced that the simultaneous increase in the NAR at $7.7 \mathrm{ka}$ is not an artifact of the sedimentation rate. In addition, $F$. profunda NAR shows several highamplitude oscillations from $7.7 \mathrm{ka}$, pointing to successive periods of high productivity in the LPZ and therefore, a change in the configuration of the upper water column may be deduced from then onwards up-core. Because all taxa were affected, even though some of them have different and/or opposite ecological preferences (e.g. nutrient availability and SST), we argue that the same ecological factors (or at least one) control them. We propose an increase in the Atlantic inflow in the Western Mediterranean Sea, as shown by the maximum flooding of the Southern coast of Spain dated at $7.4 \mathrm{ka}$ (Zazo et al., 1994), and the ensuing establishment of the WAG in the Alboran basin as a possible cause for this simultaneous peak in all NAR profiles. Directly associated with the appearance of the WAG, the productive "Malaga cell" would have been settled at that time, implying a nutrient input that would favour the blooming of all species.

It is worth noting that at $7.5 \mathrm{ka}$ the $n$-hexacosan-1-ol index records its absolute minimum coeval with low values of the total concentration of $\mathrm{C}_{37}$ alkenones (Fig. 5.4d, e), pointing to a well-ventilated deep basin. In this regard, Naranjo et al. (2012) demonstrated that the intensification of the WAG is able to ventilate deep waters in the Alboran Sea. Rohling et al. (1995) reported the establishment of the WAG at around $8 \mathrm{ka}$ from the interpretation of an abrupt faunal change in the planktic foraminiferal assemblage, also identified and dated by Pérez-Folgado et al. (2003) at $7.7 \mathrm{ka}$. A change in the benthic foraminiferal assemblage was also recognised by Melki et al. (2009) at $8 \mathrm{ka}$ in the Gulf of Lions. Jiménez-Espejo et al. (2007) deduced a remarkable redox event in the Algero-Balearic basin between 7.5 and $7 \mathrm{ka}$, interpreted as the redoxcline reaching the seabed due to intensification of the thermohaline circulation. From the analyses of pollen records, Fletcher et al. (2012) reported a long-term decline in Mediterranean forest levels from $7.5 \mathrm{ka}$. Reviewing many palaeoenvironmental records from marine and continental sites in the study area, Cortés Sánchez et al. (2012), deduced a long-term environmental crisis due to sea-level rise and changes in the thermohaline circulation at that time. Based on our results, we suggest that the $7.7 \mathrm{ka}$ event observed in the NAR records would correspond to the appearance of the "Malaga cell" as a consequence of the establishment of the WAG. Ultimately, this would have been due to a major inflowing AJ along 
with an intensification of the thermohaline circulation in the Western Mediterranean at that time.

\subsubsection{2.-Variability of the productive "Malaga cell"}

The $F$. profunda NAR and relative abundances show large oscillations from the proposed appearance of the "Malaga cell" onwards. The relative abundance of this species has classically been used as a nutricline depth indicator in palaeoceanographic reconstructions in low latitudes, where $F$. profunda shows high relative abundances and may have dominated the assemblages during some periods (Molfino y McIntyre, 1990; Beaufort et al., 1997; Flores et al., 2000; Incarbona et al., 2008). In our records, F. profunda ranges up to $8 \%$ of the total assemblage and its average contribution (3.3\%) is low, in agreement with sediment-trap analyses from the Alboran Sea (4 \% and $2.5 \%$ ) (Bárcena et al., 2004; Hernández-Almeida et al., 2011). Regarding relative abundances, the decrease/increase of one species may occur when other species increase/decrease, even though the absolute value of the former does not change. Because the changes in the relative abundance of $F$. profunda are of small magnitude, we interpret the NAR as a more suitable expression of $F$. profunda abundance and variability in this study. This species requires nutrients available in the LPZ (deeper nutricline) and low turbidity (lower productivity in the UPZ) (Ahagon et al., 1993) to allow light to reach the LPZ, and hence high values of $F$. profunda NAR can be suggested to represent these conditions. This argument is supported by the study of sediment-trap samples in the Alboran Sea, where the highest fluxes of F. profunda have been reported, along with water column stratification (Hernández-Almeida et al., 2011). Similarly, in a recent study carried out in Bay of Bengal, Mergulhao et al. (2013) linked high fluxes of $F$. profunda to a deep nutricline when oligotrophic conditions prevailed at the surface.

Conversely, the decreases in the $F$. profunda NAR were likely due to a shallower nutricline and/or higher turbulence in the UPZ, characteristic conditions of upwelling intensification. Small placoliths are a classic indicator of rich-nutrient waters in the UPZ (Okada and Wells, 1997). However, they show scarce variability from $7.7 \mathrm{ka}$ onwards and, as is the case of the relative abundance of $F$. profunda, changes of small magnitude (Fig. 5.3), and hence it is not possible to deduce periods of higher productivity from their record. Nevertheless, we argue that decreases in the $F$. profunda NAR would have been linked to the intensification of the upwelling conditions, as demonstrated by Ziveri et al. (1995) from the study of sediment-trap samples, where the lowest fluxes of $F$. profunda were linked to a shoaling of the nutricline during the upwelling period in southern California. 
From this, we deduce that the high variability shown by $F$. profunda NAR points to pulses of weakening and intensification of upwelling conditions in the study area, implying that the semipermanent nature of the productive "Malaga cell" has characterized it for the last $7.7 \mathrm{kyr}$.

Upwelling intensity in the study area is linked to AJ dynamics (Sarhan et al., 2000). This implies that more factors would have influenced productivity, since these dynamics in turn are steered by the water exchange controlled hydraulically in the Strait of Gibraltar (GarcíaLafuente et al., 2002). This water exchange determines a net water inflow at the Strait that compensates the freshwater loss at the sea-surface induced by evaporation in the Mediterranean Sea (Fenoglio-Marc et al., 2013). With regard to outflow, this is essentially formed by the LIW and WMDW. The latter occupies the bottom layer and from the study of CTD profiles, the core location has been reported to have been under its direct influence (Ercilla, 2013, personal communication). Frigola et al. (2007) analyzed the UP10 fraction (fraction coarser than $10 \mu \mathrm{m}$ ) (Fig. 5.5a) from marine core MD99-2343 (Fig. 5.1c) and interpreted the occurrence of episodes of WMDW reinforcement in the Gulf of Lions for the last $12 \mathrm{kyr}$ called the "Minorca events"(Table 5.2). There is a general correspondance between periods of maximum UP10 fraction and minimum $F$. profunda NAR, indicating a WMDW reinforcement in the Gulf of Lions coeval with intensification of the upwelling conditions in the Alboran Sea (Table 5.2, Fig. 5.5). By contrast, during most of the periods in which the UP10 fraction records its lowest values, F. profunda NAR shows large peaks, indicating a relaxation of the upwelling and a more stable water column in the Alboran Sea during periods of WMDW weakening (Fig. 5.5). The cross-correlation between the UP10 fraction and the NAR of $F$. profunda is $\mathrm{R}=-0.56, \mathrm{n}=$ 85 , $\mathrm{p}$-value $=0.01$ (correlation significant at $99 \%$ ). This result highlights the notion that both proxies are anticorrelated at a moderate value. Because Holocene climate records are imperfect proxies for processes containing complicated mixtures of periodic and random signals (Thomson, 1990), complex effects difficult to identify, isolate and remove, can affect the correlation parameter. In addition, owing to chronological uncertainties another significant concern arises when comparing records based on different age models. Thus, it is important to bear in mind that this relatively moderate correlation value results from proxies of different nature (physical and biological), which were measured in different cores from adjacent basins.

According to García-Lafuente et al. (2007), the seasonal cycle of the outflow is linked to that of the annual formation of WMDW. The same authors (2002) demonstrated that the inflow and outflow fluctuate in-phase. In light of this, it could be suggested that a reinforcement of WMDW would have been simultaneous to an enhanced Atlantic inflow in order to compensate the evaporative losses that promoted WMDW formation. Changes in the AJ velocity forced a change in its incoming direction as follows: higher velocities would have forced the AJ to 
migrate southward and, as a consequence, upwelling would have occurred offshore since a volume of uplifted waters replaced the space left behind; conversely, lower velocities would have caused the northward displacement of the AJ and the development of the WAG (Cheney y Doblar, 1982; Sarhan et al., 2000; Vargas-Yáñez et al., 2002).

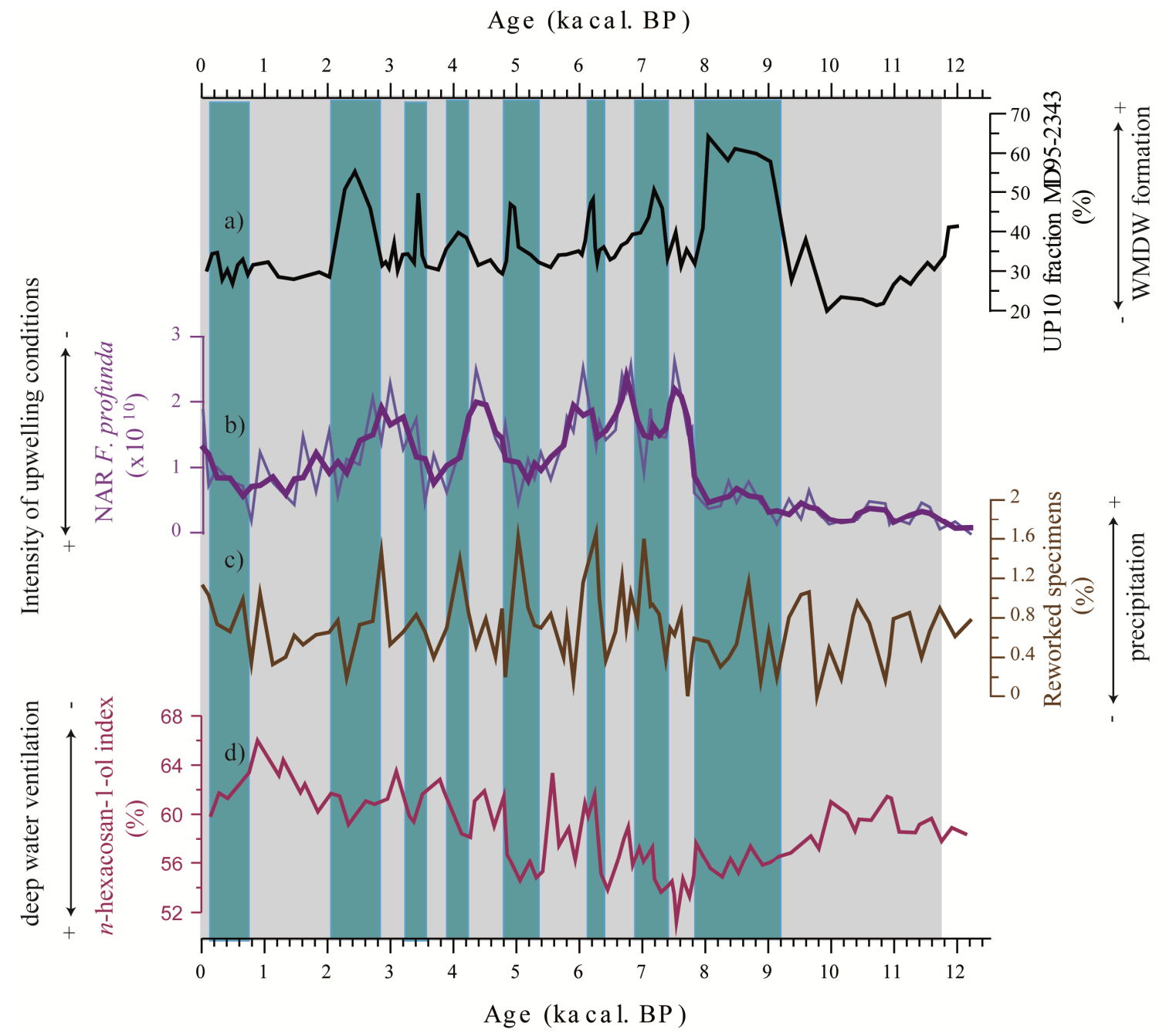

Figure 5.5. a) UP10 fraction record from core MD95-2343 north of Minorca (Frigola et al., 2007) (\%). b) F. profunda NAR from core HER-GC-T1 (coccoliths $* \mathrm{~cm}^{-2} * \mathrm{kyr}^{-1}$ ). The thin line represents original values while the thick line represents the original data fitted to a 3-point moving average smoothing spline. c) Relative abundance of reworked specimens (\%). d) $n$-hexacosan-1-ol index (\%). Blue bars represent the timing of WMDW reinforcement periods and intensified upwelling conditions in the Alboran Sea. Pale grey bars represent the timing of WMDW weakening periods along with the steadier water column deduced in this study. 
Table 5.2

Timing of Minorca Events (WMDW reinforcement) in the Gulf of Lions (Frigola et al., 2007) and timing of the lower values of $F$. profunda NAR in the Alboran Sea.

\begin{tabular}{c|cc}
\hline Minorca Event & $\begin{array}{c}\text { WMDW reinforcement periods in } \\
\text { Frigola et al. (2007) } \\
\text { Age interval (ka cal BP) }\end{array}$ & $\begin{array}{c}\text { F. profunda NAR decreases } \\
\text { in the Alboran Sea } \\
\text { Age interval (ka cal BP) }\end{array}$ \\
\hline M8 & $9.0-7.8$ & $9.2-7.8$ \\
M7 & $7.4-6.9$ & $7.4-6.9$ \\
M6 & $6.5-5.8$ & $6.6-6.1$ \\
M5 & $5.3-4.7$ & $5.7-4.7$ \\
M4 & $4.2-4.0$ & $4.2-3.1$ \\
M3 & $3.4-3.1$ & \\
M2 & $2.6-2.3$ & $2.6-2.1$ \\
M1 & $1.8-1.4$ & \\
M0 & $0.8-0.2$ & $0.9-0.2$ \\
\hline
\end{tabular}

The SST short-term variability shows changes of small amplitude (up to $1{ }^{\circ} \mathrm{C}$ ) that coincide with most of the six short cooling events $\left(1-1.5^{\circ} \mathrm{C}\right)$ recognized by Cacho et al. (2001) in a $\mathrm{U}^{\mathrm{k}^{\prime}}{ }_{37^{-}}$ SST record from the Alboran Sea. Cacho et al. (2001) studied these cooling events along a longitudinal transect, and observed that their amplitude was larger eastward. The authors argued that these were transmitted by North Atlantic inflowings but were amplified across the Mediterranean by strong winter winds. Taking into account the location of core HER-GC-T1 in this transect, the smaller magnitude of the SST cooling events is in agreement with the Mediterranean amplification effect reported by Cacho et al. (2001).

At millenial-centennial time-scale, SST and $\delta^{18} \mathrm{O}$ variations are not simultaneous. Owing to the oxygen isotope ratios within shells of G. bulloides are a function of the local temperature in which the shells forms and the variations in continental ice sheets (Emiliani, 1955), discrepancies between both records may be related to surface water salinity changes and/or ecological repercussions from the different planktic groups employed (i.e. the different depths at which G. bulloides calcifies and alkenones are produced).

The five largest peaks shown by the reworked specimens (Fig. 5.5d) may be negligible owing to their low relative abundance (up to $1.6 \%$ ). Nevertheless, we submit that they could have paleosignificance related to the processes described below due to their correspondence with periods of WMDW formation (Fig. 5.5). In the study area, reworked specimens have been linked to terrigenous input from exposed continental margins and fluctuation in the sea level 
(Flores et al., 1997; Colmenero-Hidalgo et al., 2004). We discard this hypothesis since the estimated changes in relative mean sea levels during the Holocene did not exceed $1.3 \mathrm{~m}$ in the Alboran Sea (Goy et al., 2003). Nevertheless, reworked specimens can be also transported by rivers flowing into the study area (Guadalfeo River and Guadalhorce River (Fig. 5.1b)). Southward migrations of the North Atlantic westerlies (i.e. blowing over the Gulf of Lions) allow the penetration of winter storm tracks in the Mediterranean region. This was observed for the Holocene by Fletcher et al. (2012), who linked southward displacements of the North Atlantic westerlies with forest expansion in the Western Mediterranean. Higher precipitation during times of prevailing westerlies would have involved enhanced fluvial discharges, supporting the transport of reworked specimens by rivers during periods of WMDW formation.

The several changes seen in the $n$-hexacosan-1-ol index (Fig. 5.5c) do not reveal any evident correlation with those of the UP10 fraction. It is expected that after its formation the WMDW would have ventilated the seafloor on its way to the Strait. However, fluvial discharges in the study area during those periods could have partly masked the short-term ventilation signal shown by the $n$-hexacosan-1-ol index.

From these results, we propose a two-phase scenario to explain the behavior of the productive Malaga cell at millenial-centennial time-scale from its appearance at $7.7 \mathrm{ka}$ : [1] more arid climate conditions and weaker northerlies/north-westerlies in the Gulf of Lions, the latter leading to a reduction in WMDW formation concurrent with a weakening of the AJ inflow. This would have promoted a reduction in vertical mixing (Fig. 5.6a), triggering a weakening of the upwelling conditions and leading to a more stable water column in the Alboran Sea; [2] wetter climate conditions and an intensification of northerly/north-westerly winds in the Gulf of Lions would have prompted a WMDW reinforcement simultaneous with a stronger AJ and its southward migration. This would have caused an increase in vertical mixing, leading to an intensification of the upwelling conditions in the Alboran Sea (Fig. 5.6b).

\subsection{3.-Proposed forcing mechanism}

Atmospheric pressure over the Mediterranean is the main driving force of water exchange in the Strait (García-Lafuente et al., 2002) as well as of the wind intensity, which determines the WMDW formation (Leaman y Schott, 1991; Rixen et al., 2005). On a longer time-scale, a NAO-like mode of atmospheric circulation could represent a mechanism influencing climate variability and productivity at a millenial-centennial time-scale in the study area due to the wellknown climatic teleconnection of the Western Mediterranean with northern latitudes (Cacho et al., 1999; Martrat et al., 2004). This relationship has already been suggested by several authors (Sánchez-Goñi et al., 2002; Goy et al., 2003; Moreno et al., 2004; Frigola et al., 2007; Fletcher 
et al., 2012), although the interpretations are limited by the absence of a robust paleo-NAO reconstruction. Trouet et al. (2009) reconstructed the winter-NAO circulation pattern for the past 900 years, and later, Olsen et al. (2012) expanded it to 5,200 years (Fig. 5.7). A comparison between the winter-NAO reconstruction and the UP10 fraction by Frigola et al. (2007) reveals a good visual match (Fig. 5.7b), supported by the reasonable value of its cross-correlation: $\mathrm{R}=$ $0.60, n=45, p$ value $=0.002$.
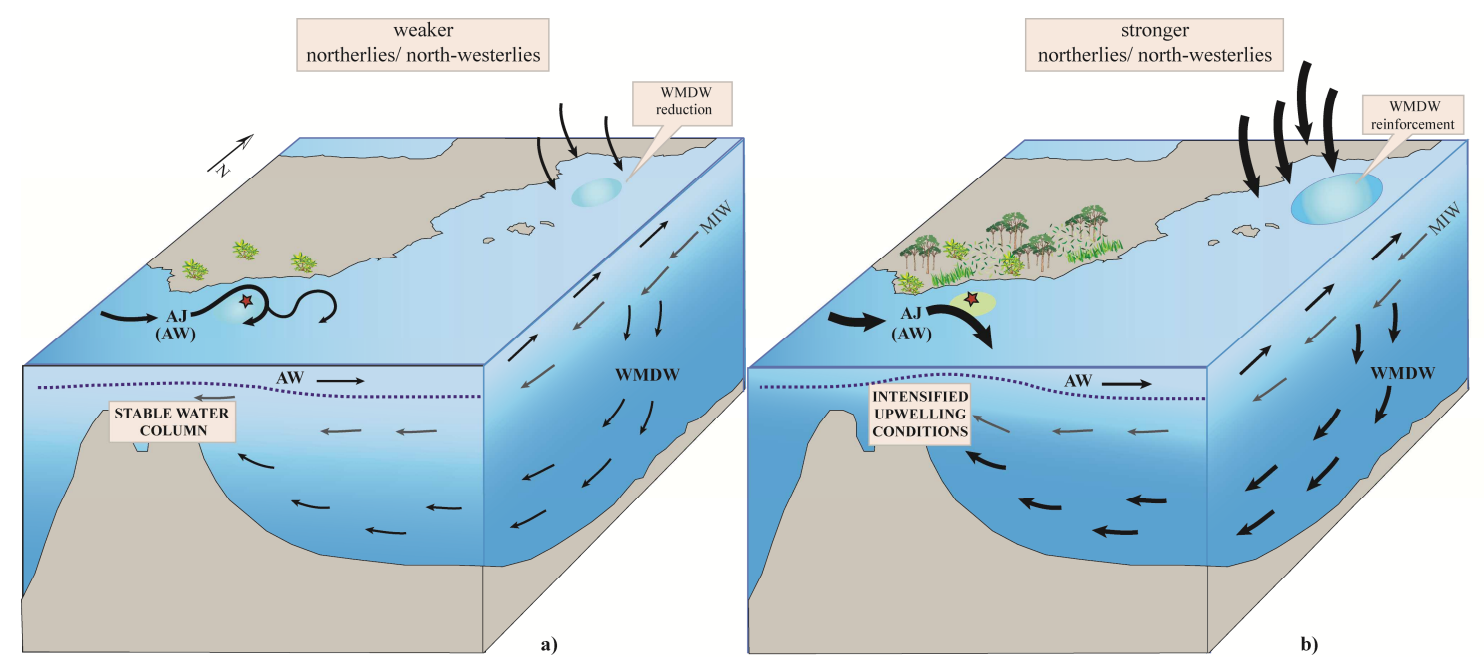

Figure 5.6. Proposed two-phase scenario: a) phase [1] and b) phase [2] as explained in the text. AW: Atlantic Water; AJ: Atlantic Jet; MIW: Intermediate Water; WMDW: Western Mediterranean Deep Water.

Trouet et al. (2009) and Olsen et al. (2012) also inferred the NAO index (Fig. 5.7a), which is the normalized December-to-March sea-level pressure between the Azores high- and the Icelandic low-pressure centers, expressed as a bipolar circulation pattern. Previous work suggests that northerly/westerly winds over the Gulf of Lions as well as enhanced precipitation in the Western Mediterranean would have been triggered during negative-like conditions (Combourieu Nebout et al., 2002; Trigo et al., 2004; Fletcher et al., 2012; Roberts et al., 2012). However, a firm relationship between the timing of periods of intensified upwelling conditions in the Alboran Sea and bimodality in the winter-NAO phases cannot be established from their comparison (Fig. 5.7, dark bars). Nevertheless, we propose the winter-NAO as a likely forcing mechanism directly involved in the intensity of northerly/north-westerly winds that drove WMDW formation, a physical process that here is in turn proposed to be related to the variability of the "Malaga cell" since its appearance at $7.7 \mathrm{ka}$. 


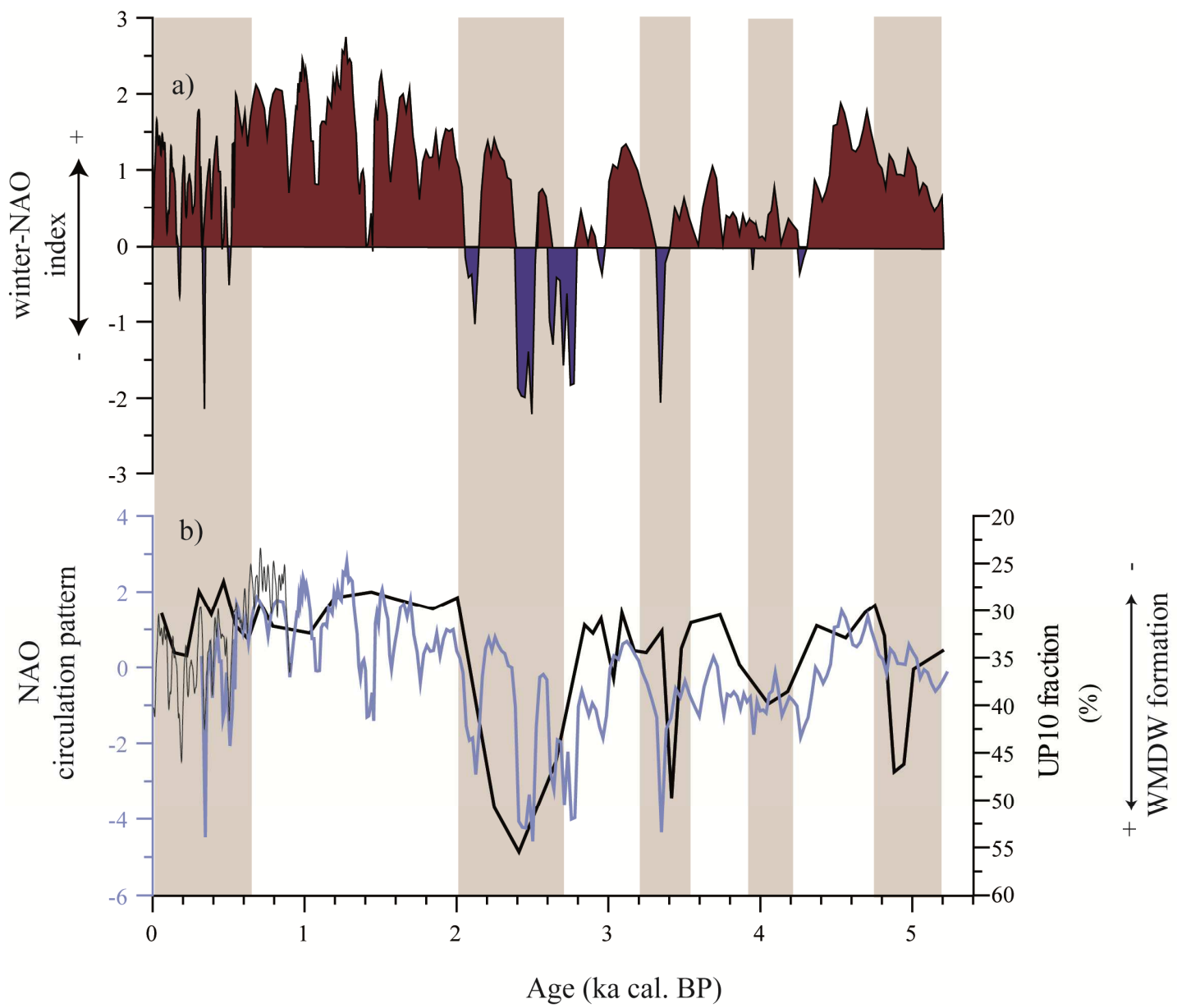

Figure 5.7. a) Winter-NAO index (Trouet et al., 2009; Olsen et al., 2012). Comparison of the winterNAO circulation pattern according to Trouet et al. (2009), in brown, and to Olsen et al. (2012), in blue, with: b) the UP10 fraction (reversed vertical axis) from core MD95-2343 (Frigola et al., 2007). Pale brown bars represent the timing of periods of WMDW reinforcement and intensified upwelling conditions in the Alboran Sea.

\section{6.-CONCLUSIONS}

Down-core variations in the NAR are tightly linked to changes in marine paleoproductivity. A dramatic change observed in the NAR of all coccolithophore taxa at $7.7 \mathrm{ka}$ has been related to a prominent fast-flowing $\mathrm{AJ}$ and the ensuing establishment of the WAG. These conditions would have favoured the establishment of the semi-permanent productive "Malaga cell" at 7.7 ka. From that time throughout the Holocene, the area was affected by a general cooling trend, as revealed by alkenone-estimated SST. Nannofossil records point to a general decreasing trend in productivity, altered by higher variability based on the alternation of events of weakened and intensified upwelling conditions since the establishment of the "Malaga cell". These variations in productivity have been found to be synchronous with periods of WMDW reinforcement in 
the Gulf of Lions, in such a way that whenever WMDW formation was strengthened, a productive event would have occurred in the Alboran Sea.

A two-phase scenario (Fig. 5.6) is proposed to describe the main climatic and oceanographic features prevailing in the Western Mediterranean along the Holocene at millennial-centennial time-scale: phase [1] together with more arid climate conditions, weaker northerly/northwesterly winds would have resulted in a reduction in WMDW formation in the Gulf of Lions, which would have coincided with a minor AJ influx. As a result, a weakening of the upwelling conditions would occur and a stable water colum would have characterized the study area; phase [2] together with wetter climate conditions, stronger northerlies/north-westerlies blowing over the Gulf of Lions would have driven a major WMDW formation simultaneous to an enhancement of the AJ influx that would have migrated southward. This would have prompted vertical mixing in the study area intensifying upwelling conditions.

The winter-NAO circulation pattern has proved to be a highly influential mechanism in the processes explained by both phases. These results highlight the sensitivity of the Western Mediterranean to high-latitude climatic systems and point to the "Malaga cell" as a location of high interest in the Alboran Sea, since it provides an information that in turn can be used to unravel the climatic and oceanographic patterns that characterized the Western Mediterranean in the past.

\section{Acknowledgements}

We thank two anonymous reviewers for their valuable contribution to improve this manuscript. B. Ausín is grateful to Y. González and B. Hortelano (Department of Environmental Chemistry, IDAEA-CSIC, Barcelona) for their guidance and supervision during the geochemical analyses. We thank G. Ercilla and J. Salat for their valuable comments and suggestions. This study was supported by FPU grant AP2010-2559 from the Ministry of Education of Spain awarded to B. Ausín and by the Consolider Ingenio "GRACCIE" CSD 2007-00067 program, the CGL2011-26493 program and the VACLIODP339 and MINECO CTM2012-38248 projects of the Spanish Ministry of Science and Innovation.

All original data in this study necessary to understand, evaluate and replicate this research are accessible via correspondence with the main author, who will willingly make them available for free to anyone upon request.

\section{References}

Abrantes F, Voelker A, Sierro FJ, Naughton F, Rodrigues T, Cacho I, Ariztegui D, Brayshaw D, Sicre M-A, Batista L y Lionello P. (2012) 1 - Paleoclimate Variability in the 
Mediterranean Region. The Climate of the Mediterranean Region. Oxford: Elsevier, 186.

Ahagon N, Tanaka Y and Ujiié H. (1993) Florisphaera profunda, a possible nannoplankton indicator of late Quaternary changes in sea-water turbidity at the northwestern margin of the Pacific. Marine Micropaleontology 22: 255-273.

Álvarez C, Amore FO, Cros L, Alonso B and Alcántara-Carrió J. (2010) Coccolithophore biogeography in the Mediterranean Iberian margin. Revista Española de Micropaleontología 42: 359-372.

Bárcena MA, Flores JA, Sierro FJ, Pérez-Folgado M, Fabres J, Calafat A and Canals M. (2004) Planktonic response to main oceanographic changes in the Alboran Sea (Western Mediterranean) as documented in sediment traps and surface sediments. Marine Micropaleontology 53: 423-445.

Baumann KH and Freitag T. (2004) Pleistocene fluctuations in the northern Benguela Current system as revealed by coccolith assemblages. Marine Micropaleontology 52: 195-215.

Baumann KH, Andruleit H, Boeckel B, Geisen M and Kinkel H. (2005) The significance of extant coccolithophores as indicators of ocean water masses, surface water temperature, and palaeoproductivity: a review. Palaeontologische Zeitschrift 79: 93-112.

Beaufort L, Lancelot Y, Camberlin P, Cayre O, Vincent E, Bassinot F and Labeyrie L. (1997) Insolation Cycles as a Major Control of Equatorial Indian Ocean Primary Production. Science 278: 1451-1454.

Berger A. (1978) Long-Term Variations of Daily Insolation and Quaternary Climatic Changes. Journal of the Atmospheric Sciences 35: 2362-2367.

Bronk Ramsey C. (2008) Deposition models for chronological records. Quaternary Science Reviews 27: 42-60.

Cacho I, Grimalt JO and Canals M. (2002) Response of the Western Mediterranean Sea to rapid climatic variability during the last 50,000 years: a molecular biomarker approach. Journal of Marine Systems 33-34: 253-272.

Cacho I, Grimalt JO, Canals M, Sbaffi L, Shackleton NJ, Schönfeld J and Zahn R. (2001) Variability of the western Mediterranean Sea surface temperature during the last 25,000 years and its connection with the Northern Hemisphere climatic changes. Paleoceanography 16: 40-52.

Cacho I, Grimalt JO, Pelejero C, Canals M, Sierro FJ, Flores JA and Shackleton N. (1999) Dansgaard-Oeschger and Heinrich Event Imprints in Alboran Sea Paleotemperatures. Paleoceanography 14: 698-705. 
Cacho I, Grimalt JO, Sierro FJ, Shackleton N and Canals M. (2000) Evidence for enhanced Mediterranean thermohaline circulation during rapid climatic coolings. Earth and Planetary Science Letters 183: 417-429.

Cañellas B, Orfila Förster A, Méndez Incera FJ, Álvarez Díaz A and Tintoré Subirana J. (2010) Influence of the NAO on the northwestern Mediterranean wave climate. Scientia Marina 74: 55-64.

Colmenero-Hidalgo E, Flores JA, Sierro FJ, Bárcena MÁ, Löwemark L, Schönfeld J and Grimalt JO. (2004) Ocean surface water response to short-term climate changes revealed by coccolithophores from the Gulf of Cadiz (NE Atlantic) and Alboran Sea (W Mediterranean). Palaeogeography, Palaeoclimatology, Palaeoecology 205: 317-336.

Combourieu Nebout N, Turon JL, Zahn R, Capotondi L, Londeix L and Pahnke K. (2002) Enhanced aridity and atmospheric high-pressure stability over the western Mediterranean during the North Atlantic cold events of the past 50 ky Geology 30: 863866.

Coplen TB. (1996) Editorial: More uncertainty than necessary. Paleoceanography 11: 369-370.

Cortés Sánchez M, Jiménez Espejo FJ, Simón Vallejo MD, Gibaja Bao JF, Carvalho AF, Martínez-Ruiz F, Gámiz MR, Flores JA, Paytan A, López Sáez JA, Peña-Chocarro L, Carrión JS, Morales Muñiz A, Roselló Izquierdo E, Riquelme Cantal JA, Dean RM, Salgueiro E, Martínez Sánchez RM, De la Rubia de Gracia JJ, Lozano Francisco MC, Vera Peláez JL, Rodríguez LL and Bicho NF. (2012) The Mesolithic-Neolithic transition in southern Iberia. Quaternary Research 77: 221-234.

Cheney RE and Doblar RA. (1982) Structure and variability of the Alboran sea frontal system. Journal of Geophysical Research: Oceans 87: 585-594.

Dafner EV, Boscolo R and Bryden HL. (2003) The N:Si:P molar ratio in the Strait of Gibraltar. Geophysical Research Letters 30: 1506.

Emiliani C. (1955) Pleistocene temperatures. Geology 63: 539-578.

Fatela F and Taborda R. (2002) Confidence limits of species proportions in microfossil assemblages. Marine Micropaleontology 45: 169-174.

Fenoglio-Marc L, Mariotti A, Sannino G, Meyssignac B, Carillo A, Struglia MV and M; R. (2013) Decadal variability of net water flux at the Mediterranean Sea Gibraltar Strait. Global and Planetary Change 100: 1-10.

Fletcher WJ, Debret M and Sánchez Goñi MF. (2012) Mid-Holocene emergence of a lowfrequency millennial oscillation in western Mediterranean climate: Implications for past dynamics of the North Atlantic atmospheric westerlies. The Holocene 23: 153-166.

Fletcher WJ and Zielhofer C. (2011) Fragility of Western Mediterranean landscapes during Holocene Rapid Climate Changes. CATENA. 
Flores JA, Bárcena MA and Sierro FJ. (2000) Ocean-surface and wind dynamics in the Atlantic Ocean off Northwest Africa during the last 140000 years. Palaeogeography, Palaeoclimatology, Palaeoecology 161: 459-478.

Flores JA, Gersonde R and Sierro FJ. (1999) Pleistocene fluctuations in the Agulhas Current Retroflection based on the calcareous plankton record. Marine Micropaleontology 37: $1-22$.

Flores JA and Marino M. (2002) Pleistocene calcareous nannofossil stratigraphy for ODP Leg 177 (Atlantic sector of the Southern Ocean) Marine Micropaleontology 45: 191-224.

Flores JA and Sierro FJ. (1997) Revised technique for calculation of calcareous nannofossil accumulation rates. Micropaleontology 43: 321-324.

Flores JA, Sierro FJ, Francés G, Vázquez A and Zamarreño I. (1997) The last 100,000 years in the western Mediterranean: sea surface water and frontal dynamics as revealed by coccolithophores. Marine Micropaleontology 29: 351-366.

Font J, Palanques A, Puig P, Salat J and Emelianov M. (2007) Sequence of hydrographic changes in NW Mediterranean deep water due to the exceptional winter of 2005. Scientia Marina 71: 339-346.

Frigola J, Moreno A, Cacho I, Canals M, Sierro FJ, Flores JA, Grimalt JO, Hodell DA and Curtis JH. (2007) Holocene climate variability in the western Mediterranean region from a deepwater sediment record. Paleoceanography 22: PA2209.

García-Gorriz E and Carr ME. (1999) The climatological annual cycle of satellite-derived phytoplankton pigments in the Alboran Sea: a physical interpretation. Geophysical Research Letters 26: 2985-2988.

García-Lafuente J, Álvarez Fanjul E, Vargas JM and Ratsimandresy AW. (2002) Subinertial variability in the flow through the Strait of Gibraltar. Journal of Geophysical Research: Oceans 107 (C10): 3168.

García-Lafuente J, Sánchez Román A, Díaz del Río G, Sannino G and Sánchez Garrido JC. (2007) Recent observations of seasonal variability of the Mediterranean outflow in the Strait of Gibraltar. Journal of Geophysical Research: Oceans 112: C10005.

García-Lafuente J, Vargas JM, Plaza F, Sarhan T, Candela J and Bascheck B. (2000) Tide at the eastern section of the Strait of Gibraltar. Journal of Geophysical Research: Oceans 105: 14197-14213.

Giraudeau J. (1992) Distribution of Recent nannofossils beneath the Benguela system: Southwest African continental margin. Marine Geology 108: 219-237.

Goy JL, Zazo C and Dabrio CJ. (2003) A beach-ridge progradation complex reflecting periodical sea-level and climate variability during the Holocene (Gulf of Almería, Western Mediterranean). Geomorphology 50: 251-268. 
Hammer Ø, Harper, D.A.T and Ryan, P.D. (2001) PAST: Paleontological statistics software package for education and data analysis. Palaeontologia Electronica 4: 9.

Heburn GW and La Violette PE. (1990) Variations in the Structure of the Anticyclonic Gyres Found in the Alboran Sea. Journal of Geophysical Research 95: 1599-1613.

Hernández-Almeida I, Bárcena MA, Flores JA, Sierro FJ, Sánchez-Vidal A and Calafat A. (2011) Microplankton response to environmental conditions in the Alboran Sea (Western Mediterranean): One year sediment trap record. Marine Micropaleontology 78: $14-24$.

Hurrell JW. (1995) Decadal Trends in the North Atlantic Oscillation: Regional Temperatures and Precipitation. Science 269: 676-679.

Incarbona A, Di Stefano E, Patti B, Pelosi N, Bonomo S, Mazzola S, Sprovieri R, Tranchida G, Zgozi S and Bonanno A. (2008) Holocene millennial-scale productivity variations in the Sicily Channel (Mediterranean Sea). Paleoceanography 23: PA3204.

IPCC. (2013) IPCC, 2013: Climate Change 2013: The Physical Science Basis. Contribution of Working Group I to the Fifth Assessment Report of the Intergovernmental Panel on Climate Change In: Stocker TF, D. Qin, G.K. Plattner, M. Tignor, S.K. Allen, J. Boschung, A. Nauels, Y. Xia, V. Bex and P.M. Midgley (ed) Cambridge University Press. Cambridge, United Kingdom and New York, NY, USA, 1535 pp.

Jalut G, Esteban Amat A, Bonnet L, Gauquelin T and Fontugne M. (2000) Holocene climatic changes in the Western Mediterranean, from south-east France to south-east Spain. Palaeogeography, Palaeoclimatology, Palaeoecology 160: 255-290.

Jalut G, Esteban Amat A, Riera i Mora S, Fontugne M, Mook R, Bonnet L and Gauquelin T. (1997) Holocene climatic changes in the western Mediterranean: installation of the Mediterranean climate. Comptes Rendus de l'Académie des Sciences - Series IIA - Earth and Planetary Science 325: 327-334.

Jiménez-Espejo FJ, Martínez-Ruiz F, Sakamoto T, Lijima K, Gallego-Torres D and Harada N. (2007) Paleoenvironmental changes in the western Mediterranean since the last glacial maximum: High resolution multiproxy record from the Algero-Balearic basin. Palaeogeography, Palaeoclimatology, Palaeoecology 246: 292-306.

Josey SA, Somot S and Tsimplis M. (2011) Impacts of atmospheric modes of variability on Mediterranean Sea surface heat exchange. Journal of Geophysical Research: Oceans 116: $\mathrm{C} 02032$.

Leaman KD and Schott FA. (1991) Hydrographic Structure of the Convection Regime in the Gulf of Lions: Winter 1987. Journal of Physical Oceanography 21: 575-598.

Marchal O, Cacho I, Stocker TF, Grimalt JO, Calvo E, Martrat B, Shackleton N, Vautravers M, Cortijo E, van Kreveld S, Andersson C, Koç N, Chapman M, Sbaffi L, Duplessy JC, 
Sarnthein M, Turon JL, Duprat J and Jansen E. (2002) Apparent long-term cooling of the sea surface in the northeast Atlantic and Mediterranean during the Holocene. Quaternary Science Reviews 21: 455-483.

Martrat B, Grimalt JO, Lopez-Martínez C, Cacho I, Sierro FJ, Flores JA, Zahn R, Canals M, Curtis JH and Hodell DA. (2004) Abrupt Temperature Changes in the Western Mediterranean over the Past 250,000 Years. Science 306: 1762-1765.

Mayewski PA, Rohling EE, Curt Stager J, Karlén W, Maasch KA, David Meeker L, Meyerson EA, Gasse F, van Kreveld S, Holmgren K, Lee-Thorp J, Rosqvist G, Rack F, Staubwasser M, Schneider RR and Steig EJ. (2004) Holocene climate variability. Quaternary Research 62: 243-255.

McIntyre A and Bé AWH. (1967) Modern coccolithophoridae of the Atlantic Ocean. Placoliths and cyrtoliths. Deep Sea Research and Oceanographic Abstracts 14: 561-597.

MEDOCGROUP. (1970) Observation of formation of deep water in the Mediterranean Sea, 1969. Nature 227: 1037-1040.

Melki T, Kallel N, Jorissen FJ, Guichard F, Dennielou B, Berné S, Labeyrie L and Fontugne M. (2009) Abrupt climate change, sea surface salinity and paleoproductivity in the western Mediterranean Sea (Gulf of Lion) during the last 28 kyr. Palaeogeography, Palaeoclimatology, Palaeoecology 279: 96-113.

Mergulhao LP, Guptha MVS, Unger D and Murty VSN. (2013) Seasonality and variability of coccolithophore fluxes in response to diverse oceanographic regimes in the Bay of Bengal: Sediment trap results. Palaeogeography, Palaeoclimatology, Palaeoecology 371: 119-135.

Mertens C and Schott F. (1998) Interannual Variability of Deep-Water Formation in the Northwestern Mediterranean. Journal of Physical Oceanography 28: 1410-1424.

Millot C. (1999) Circulation in the Western Mediterranean Sea. Journal of Marine Systems 20: 423-442.

Minas HJ, Coste B, Le Corre P, Minas M and Raimbault P. (1991) Biological and Geochemical Signatures Associated With the Water Circulation Through the Strait of Gibraltar and in the Western Alboran Sea. Journal of Geophysical Research 96: 8755-8771.

Molfino B and McIntyre A. (1990) Precessional forcing of nutricline dynamics in the equatorial Atlantic. Science 249: 766-769.

Moreno A, Cacho I, Canals M, Grimalt JO and Sánchez-Vidal A. (2004) Millennial-scale variability in the productivity signal from the Alboran Sea record, Western Mediterranean Sea. Palaeogeography, Palaeoclimatology, Palaeoecology 211: 205219. 
Müller PJ, Kirst G, Ruhland G, von Storch I and Rosell-Melé A. (1998) Calibration of the alkenone paleotemperature index U37K' based on core-tops from the eastern South Atlantic and the global ocean $\left(60^{\circ} \mathrm{N}-60^{\circ} \mathrm{S}\right)$. Geochimica et Cosmochimica Acta 62 : 1757-1772.

Naranjo C, García-Lafuente J, Sánchez Garrido JC, Sánchez Román A and Delgado Cabello J. (2012) The Western Alboran Gyre helps ventilate the Western Mediterranean Deep Water through Gibraltar. Deep Sea Research Part I: Oceanographic Research Papers 63: $157-163$.

Okada H and Honjo S. (1973) The distribution of oceanic coccolithophorids in the Pacific. Deep Sea Research and Oceanographic Abstracts 20: 355-374.

Olsen J, Anderson NJ y Knudsen MF. (2012) Variability of the North Atlantic Oscillation over the past 5,200 years. Nature Geosciences 5: 808-812.

Paillard D, Labeyrie L and Yiou P. (1996) Macintosh program performs time-series analysis. Eos Transactions American Geophysical Union 77: 379.

Reimer PJ, Bard E, Bayliss A, Beck JW, Blackwell PG, Bronk Ramsey C, Grootes PM, Guilderson TP, Haflidason H, Hajdas I, HattŽ C, Heaton TJ, Hoffmann DL, Hogg AG, Hughen KA, Kaiser KF, Kromer B, Manning SW, Niu M, Reimer RW, Richards DA, Scott EM, Southon JR, Staff RA, Turney CSM and Van der Plicht J. (2013) IntCal13 and Marine13 Radiocarbon Age Calibration Curves 0-50,000 Years cal BP. Radiocarbon, Radiocarbon 55.

Rixen M, Beckers JM, Levitus S, Antonov J, Boyer T, Maillard C, Fichaut M, Balopoulos E, Iona S, Dooley H, García MJ, Manca B, Giorgetti A, Manzella G, Mikhailov N, Pinardi N and Zavatarelli M. (2005) The Western Mediterranean Deep Water: A proxy for climate change. Geophysical Research Letters 32: L12608.

Roberts N, Moreno A, Valero-Garcés BL, Corella JP, Jones M, Allcock S, Woodbridge J, Morellón M, Luterbacher J, Xoplaki E and Türkes M. (2012) Palaeolimnological evidence for an east-west climate see-saw in the Mediterranean since AD 900. Global and Planetary Change 84-85: 23-34.

Rohling EJ, Den Dulk M, Pujol C and Vergnaud-Grazzini C. (1995) Abrupt hydrographic change in the Alboran Sea (western Mediterranean) around 8000 yrs BP. Deep Sea Research Part I: Oceanographic Research Papers 42: 1609-1619.

Sánchez-Goñi M, Cacho I, Turon J, Guiot J, Sierro FJ, Peypouquet J, Grimalt J and Shackleton N. (2002) Synchroneity between marine and terrestrial responses to millennial scale climatic variability during the last glacial period in the Mediterranean region. Climate Dynamics 19: 95-105. 
Sarhan T, García-Lafuente J, Vargas M, Vargas JM and Plaza F. (2000) Upwelling mechanisms in the northwestern Alboran Sea. Journal of Marine Systems 23: 317-331.

Siani G, Paterne M, Arnold M, Bard E, Métivier B, Tisnerat N and Bassinot F. (2000) Radiocarbon reservoir ages in the Mediterranean Sea and Black Sea. Radiocarbon 42: 271-280.

Sierro FJ, Hodell DA, Curtis JH, Flores JA, Reguera I, Colmenero-Hidalgo E, Bárcena MA, Grimalt JO, Cacho I, Frigola J and Canals M. (2005) Impact of iceberg melting on Mediterranean thermohaline circulation during Heinrich events. Paleoceanography 20: PA2019.

Smith RO, Bryden HL and Stansfield K. (2008) Observations of new western Mediterranean deep water formation using Argo floats 2004-2006. Ocean Science 2: 133-149.

Stuiver M and Braziunas TF. (1993) 14C Ages of Marine Samples to 10,000 BC Radiocarbon 35: 137-118.

Sumner G, Homar V and Ramis C. (2001) Precipitation seasonality in eastern and southern coastal Spain. International Journal of Climatology 21: 219-247.

Thierstein HR and Young JR. (2004) Coccolithophores: from molecular processes to global impact. Springer, Berlin (565 pp.).

Thomson DJ. (1990) Time Series Analysis of Holocene Climate Data. Philosophical Transactions of the Royal Society of London. Series A, Mathematical and Physical Sciences 330: 601-616.

Trigo RM, Pozo-Vázquez D, Osborn TJ, Castro-Díez Y, Gámiz-Fortis S and Esteban-Parra MJ. (2004) North Atlantic oscillation influence on precipitation, river flow and water resources in the Iberian Peninsula. International Journal of Climatology 24: 925-944.

Trigo R, Xoplaki E, Zorita E, Luterbacher J, Krichak SO, Alpert P, Jacobeit J, Sáenz J, Fernández J, González-Rouco F, García-Herrera R, Rodo X, Brunetti M, Nanni T, Maugeri M, Türke§ M, Gimeno L, Ribera P, Brunet M, Trigo IF, Crepon M, Mariotti A, P. Lionello PMR y Boscolo R. (2006) Chapter 3 Relations between variability in the Mediterranean region and mid-latitude variability. Developments in Earth and Environmental Sciences. Elsevier, 179-226.

Trouet V, Esper J, Graham NE, Baker A, Scourse JD and Frank DC. (2009) Persistent Positive North Atlantic Oscillation Mode Dominated the Medieval Climate Anomaly Science 324: $78-80$.

Tsimplis MN and Josey SA. (2001) Forcing of the Mediterranean Sea by atmospheric oscillations over the North Atlantic. Geophysical Research Letters 28: 803-806. 
Vargas-Yáñez M, Plaza F, García-Lafuente J, Sarhan T, Vargas JM and Vélez-Belchi P. (2002) About the seasonal variability of the Alboran Sea circulation. Journal of Marine Systems 35: 229-248.

Villanueva J, Pelejero C and Grimalt JO. (1997) Clean-up procedures for the unbiased estimation of $\mathrm{C}_{37}$ alkenone sea surface temperatures and terrigenous n-alkane inputs in paleoceanography. Journal of Chromatography A 757: 145-151.

Wanner H, Solomina O, Grosjean M, Ritz SP and Jetel M. (2011) Structure and origin of Holocene cold events. Quaternary Science Reviews 30: 3109-3123.

Weaver PPE and Pujol C. (1988) History of the last deglaciation in the Alboran Sea (western Mediterranean) and adjacent north Atlantic as revealed by coccolith floras. Palaeogeography, Palaeoclimatology, Palaeoecology 64: 35-42.

Zazo C, Goy JL, Somoza L, Dabrio CJ, Belluomini G, Improta S, Lario J, Bardají T and Silva PG. (1994) Holocene sequence of sea-level fluctuations in relation to climatic trends in the Atlantic-Mediterranean linkage coast. Journal of Coastal Research 10: 933-945.

Ziveri P, Thunell RC and Rio D. (1995) Export production of coccolithophores in an upwelling region: Results from San Pedro Basin, Southern California Borderlands. Marine Micropaleontology 24: 335-358. 

CAPÍTULO 6 



\section{6.-HIGH-RESOLUTION SEA SURFACE SALINITY RECONSTRUCTION FOR THE \\ LAST 25 KYR IN THE WESTERN MEDITERRANEAN FROM A \\ COCCOLITHOPHORE-BASED TRANSFER FUNCTION}

B. Ausín (b_ausin@usal.es); I. Hernández-Almeida ${ }^{\mathrm{b}}$ (ivan.hernandez@giub.unibe.ch); J-A. Flores $^{\mathrm{a}} \quad$ (flores@usal.es); $\quad$ F-J. $\quad$ Sierro $^{\mathrm{a}} \quad$ (sierro@usal.es); $\quad$ M. $\quad$ Grosjean $^{\mathrm{b}}$ (martin.grosjean@oeschger.unibe.ch); G. Francés ${ }^{c} \quad$ (gfrances@uvigo.es); B. Alonso ${ }^{\mathrm{d}}$ (belen@icm.csic.es).

${ }^{a}$ Department of Geology, University of Salamanca, La Merced s/n, 37008 Salamanca, Spain. Spain.

${ }^{\mathrm{b}}$ Institute of Geography and Oeschger Centre for Climate Change Research, University of Bern, Erlachstrasse 9a, 3012 Bern, CH-3012 Bern, Switzerland.

'Department of Marine Geosciences, University of Vigo, Campus As Lagoas - Marcosende, 36310 Vigo, Spain.

${ }^{\mathrm{d}}$ Department of Marine Geosciences, Instituto de Ciencias del Mar (CSIC), Passeig Marítim de la Barceloneta, 37-49. E-08003 Barcelona, Spain.

\section{Corresponding author:}

Blanca Ausín (b_ausin@usal.es)

Pza/ La Merced s/n, 37008 Salamanca, Spain.

Tel: (+34) 923294497

Fax: (+34) 923294514

Under review in Quaternary Science Reviews 


\section{Resumen}

Se estudió un nuevo conjunto de datos compuesto por 89 muestras de sedimento superficial y varias de las variables oceánicas correspondientes a las mismas (temperatura, salinidad, clorofila- $a$, oxígeno, etc.) para cuantificar la relación entre las asociaciones de cocolitóforos y las condiciones ambientales modernas en el mar Mediterráneo occidental y el lado atlántico del estrecho de Gibraltar. Los análisis estadísticos multivariantes revelaron que las especies de cocolitóforos estaban mayormente relacionadas con el gradiente de salinidad marina superficial $(\mathrm{SSS})^{5}$, la cual explica una proporción independiente y significativa de la varianza en los datos de recuentos de cocolitóforos. Se desarrolló una función de transferencia basada en cocolitóforos para estimar la SSS usando la técnica de análogos modernos (MAT) y la regresión parcial por mínimos cuadrados ponderados (WA-PLS). El coeficiente de regresión obtenido

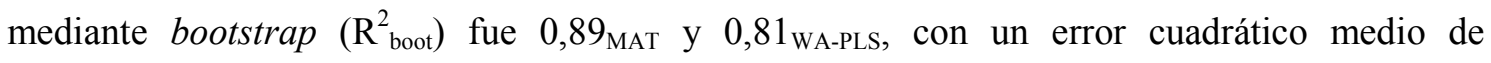

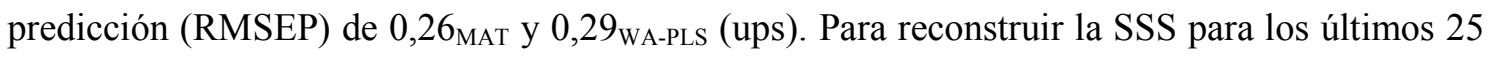
ka, la función de transferencia resultante se aplicó a las asociaciones fósiles de cocolitóforos en el testigo de sedimento CEUTA10PC08 del mar de Alborán (Mediterráneo occidental) con una resolución de $\sim 70$ años. La fiabilidad de la reconstrucción se evaluó mediante el estudio del grado de similitud entre las asociaciones cocolitoforales fósiles y las modernas y mediante la comparación con otros registros de SSS de zonas cercanas. Varios cambios de SSS a escala milenaria y secular fueron observados para los últimos $25 \mathrm{ka}$, asociados con variaciones en el influjo de agua atlántica, oscilaciones del nivel del mar y condiciones atmosféricas áridas o húmedas en el Mediterráneo occidental. El Último Máximo Glacial se caracterizó por valores más altos de SSS probablemente debido a una conexión más limitada entre el Atlántico y el Mediterráneo. Como resultado del descenso de SSS entre 15 y $13 \mathrm{ka}$, se propone que la consecuente reducción en la densidad del agua superficial y el aumento en la estratificación de la columna de agua pudieron promover la formación de la Organic-Rich Layer. A partir del Younger Dryas hasta el Holoceno, seis descensos bruscos de SSS fueron correlacionados con breves periodos de enfriamiento en el mar de Alborán y ligados a la inyección de agua noratlántica de deshielo y a condiciones continentales áridas en el Mediterráneo occidental.

Palabras clave: función de transferencia, cocolitóforos, salinidad, Último Máximo Glacial, mar Mediterráneo occidental.

\footnotetext{
${ }^{5}$ Los acrónimos siguen la nomenclatura anglosajona por razones de consistencia con el resto del texto.
} 


\begin{abstract}
A new dataset of 89 marine surface sediment samples and related oceanic environmental variables (temperature, salinity, chlorophyll- $a$, oxygen, etc.) was studied to quantify the relationship between assemblages of coccolithophore species and modern environmental conditions in the Western Mediterranean Sea and the Atlantic side of the Strait of Gibraltar. Multivariate statistical analyses revealed that coccolithophore species were primarily related to the sea surface salinity (SSS) gradient, which explains an independent and significant proportion of variance in the coccolithophore data. A quantitative coccolithophore-based transfer function to estimate SSS was developed using the Modern Analog Technique (MAT) and weighted-averaging partial-least square regression (WA-PLS). The bootstrapped regression

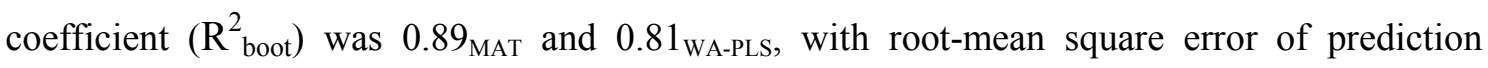
(RMSEP) of $0.26_{\mathrm{MAT}}$ and $0.29_{\text {WA-PLS }}$ (psu). In order to reconstruct SSS for the last $25 \mathrm{kyr}$, the resulting transfer function was applied to fossil coccolithophore assemblages in the highly resolved $(\sim 70$ yr) sediment core CEUTA10PC08 from the Alboran Sea (Western Mediterranean). The reliability of the reconstruction was evaluated by assessing the degree of similarity between fossil and modern coccolithophore assemblages and through comparisons with other published SSS records from nearby cores. Several millennial and centennial SSS changes were observed for the last $25 \mathrm{kyr}$, associated with variations in the Atlantic Water entering the Alboran Sea, sea-level oscillations, and arid or humid atmospheric conditions in the Western Mediterranean. The Last Glacial Maximum was characterized by higher SSS values likely due to a more limited Atlantic-Mediterranean connection. As a result of the decrease in SSS from 15 to $13 \mathrm{ka}$, a reduction in sea surface density and an increase in water column stratification are suggested to have triggered the formation of the Organic-Rich Layer. From the Younger Dryas to the Holocene, six abrupt SSS decreases were correlated with brief cooling periods in the Alboran Sea, linked to the injection of North Atlantic meltwater and continental aridity in the Western Mediterranean.
\end{abstract}

Key words: transfer function; coccolithophores; salinity; Last Glacial Maximum; Western Mediterranean Sea. 


\section{1.-INTRODUCTION}

After the Climate Long-Range Investigation Mapping and Prediction (CLIMAP) project used transfer functions to reconstruct global sea surface temperatures (SST) at $18 \mathrm{ka}$ (CLIMAP Project Members, 1976), this method has been routinely employed in an increasing number of paleoceanographic studies using microfossil census counts, such as planktonic foraminera, diatoms, radiolaria and dinoflagellates. Transfer functions are based on the calibration of the modern relationship between organisms and environmental conditions, and this information is in turn used to reconstruct past environmental variables. Unlike other marine organisms, coccolithophores have not been used systematically to develop transfer functions, even though they are one of the major components of phytoplankton. They are sensitive to coastal currents, gyres, upwelling episodes, and fluvial discharges (Baumann et al., 2005; Guerreiro et al., 2013) and their fossil coccoliths are widely used in qualitative paleoenvironmental studies. However, due to the ecological intricacies of coccolithophore species, the relationships of these organisms with their environment have barely been explored and are poorly understood. Different statistical approaches based on coccolithophores have been proposed in order to generate quantitative paleoreconstructions of different ecological variables. Giraudeau and Rogers (1994) used factor analyses and multiple regressions to estimate chlorophyll $a$ from coccolithophore census counts in surface sediment samples in the Benguela upwelling area. Several authors (Beaufort et al., 1997; Beaufort et al., 2001; Incarbona et al., 2008) calibrated the relative abundance of the coccolithophore Floriphaera profunda in surface sediment samples with respect to primary productivity and reconstructed past variations of this parameter in the Indian and Pacific oceans and in the central Mediterranean Sea. Saavedra-Pellitero et al. (2011, 2013) used linear regressions methods to derive past SST estimates in the southeast Pacific Ocean from coccolithophore census counts and accumulation rates. Bollmann and Herrle (2007) and Bollmann et al. (2009) applied multiple linear regressions to morphometric measurements of the coccolithophore Emiliania huxleyi from globally distributed core-top and plankton samples to obtain modern and past sea surface salinity (SSS) estimates.

To date, no coccolithophore-based transfer function has been applied in the Mediterranean Sea. In this confined basin, the estimation of SST and SSS changes is essential for determining Atlantic-Mediterranean water mass exchange through the Strait of Gibraltar. This exchange depends on variations in the hydrological cycle, ice-volume effects, and Mediterranean circulation patterns, which have a thermohaline origin. While quantitative SST paleoreconstructions using planktonic foraminifers and alkenones are common in the Mediterranean Sea (Cacho et al., 1999; Pérez-Folgado et al., 2003; Martrat et al., 2004; Martrat et al., 2007; Rodrigo-Gámiz et al., 2014), quantitative estimations of SSS are scarce (e.g. Kallel et al., 1997; Emeis et al., 2000; Essallami et al., 2007). The most common method is based on a 
two-step procedure: the conversion of foraminiferal $\delta^{18} \mathrm{O}$ in seawater $\delta^{18} \mathrm{O}$ through an independent SST record, followed by estimation of the salinity from its relationship with seawater $\delta^{18} \mathrm{O}$, which is approximated to its modern slope since it is unknown for the past. This technique depends strongly on the accuracy of the SST (Schmidt, 1998). Moreover, Rohling (1999) has demonstrated that in the Mediterranean context salinity and seawater $\delta^{18} \mathrm{O}$ have shown non-proportional responses to environmental changes over the past 20,000 yr. Consequently, this method is sensitive to several deviations and uncertainties (Rohling and Bigg, 1998; Schmidt, 1999; Rohling, 2000). Methods other than those currently used to estimate SSS such as the above ones would help to define salinity variations and changes in the Mediterranean water budget in the past (Rohling and Bigg, 1998; Schmidt, 1998). This would be a great opportunity for climate modeling purposes and especially, it would broaden our understanding of the Mediterranean climate in the past, where salinity changes were particularly relevant owing to the confined nature of this basin.

The aim of this study is to explore the potential of coccolithophores for the development of quantitative reconstructions in the Mediterranean Sea. We study the response of coccolithophore assemblages from surface sediment samples from Atlantic Ocean and Mediterranean Sea to environmental variables, such as SST and SSS. The resulting calibration model (transfer function) for SSS was used to reconstruct SSS changes at high-resolution in the Alboran Sea (Western Mediterranean) for the last $25 \mathrm{kyr}$. The reliability of the reconstruction was assessed by analysis of the similarity between fossil and modern coccolithophore assemblages and by comparison with other SSS records from nearby locations obtained from independent proxies. Finally, centennial and millennial SSS changes are described and discussed.

\section{2.-STUDY AREA AND GENERAL SURFACE CIRCULATION}

The Western Mediterranean Sea is a semi-enclosed basin situated at mid-latitudes (Fig. 6.1a). In this region evaporation exceeds precipitation plus runoff, such that water budgets tend to be balanced by the advection of relatively less saline Atlantic Water (AW) through the Strait of Gibraltar (Bèthoux, 1979).

The Alboran Sea located in the Western Mediterranean (Fig. 6.1a) is a transitional region where the AW flows eastward while mixing with Mediterranean water to form the Modified Atlantic Water (MAW) at the surface (100-200 m) (Millot, 1999). This distinctive feature affects the spatial distribution of some environmental parameters such as SST and SSS, leading to the existence of well-defined horizontal gradients in the Western Mediterranean in annual terms. Strong seasonal variability alters some of these gradients along the year: insolation in summer and early-fall causes a stratification of the water column, while during winter, vertical mixing is intense (Dafner et al., 2003). By contrast, the absolute values of other gradients such 
as SSS (which ranges from 36.2 practical salinity units (psu) near the Strait to $38.2 \mathrm{psu}$ in the Gulf of Lions) and their spatial distribution remain constant throughout the year (Fig. 6.1b) (WOA 13) (Zweng et al., 2013). The MAW flows along the Algerian coast and continues its path eastward to the Tyrrhenian Sea and then northward, to the Gulf of Lions. As a result, the MAW becomes warmer and more saline, (it is renamed the Northern Current (NC)), and flows southward along the Catalan-Balearic Sea (Millot, 1992). The morphology of the CatalanBalearic continental slopes modifies its direction, forming mesoscale eddies (Font et al., 1995). The NC is separated from less saline coastal waters by the Catalan Front (Fig. 6.1a) and from the younger MAW (less warm and fresher) by the Balearic Front (Font et al., 1988) (Fig. 6.1a).

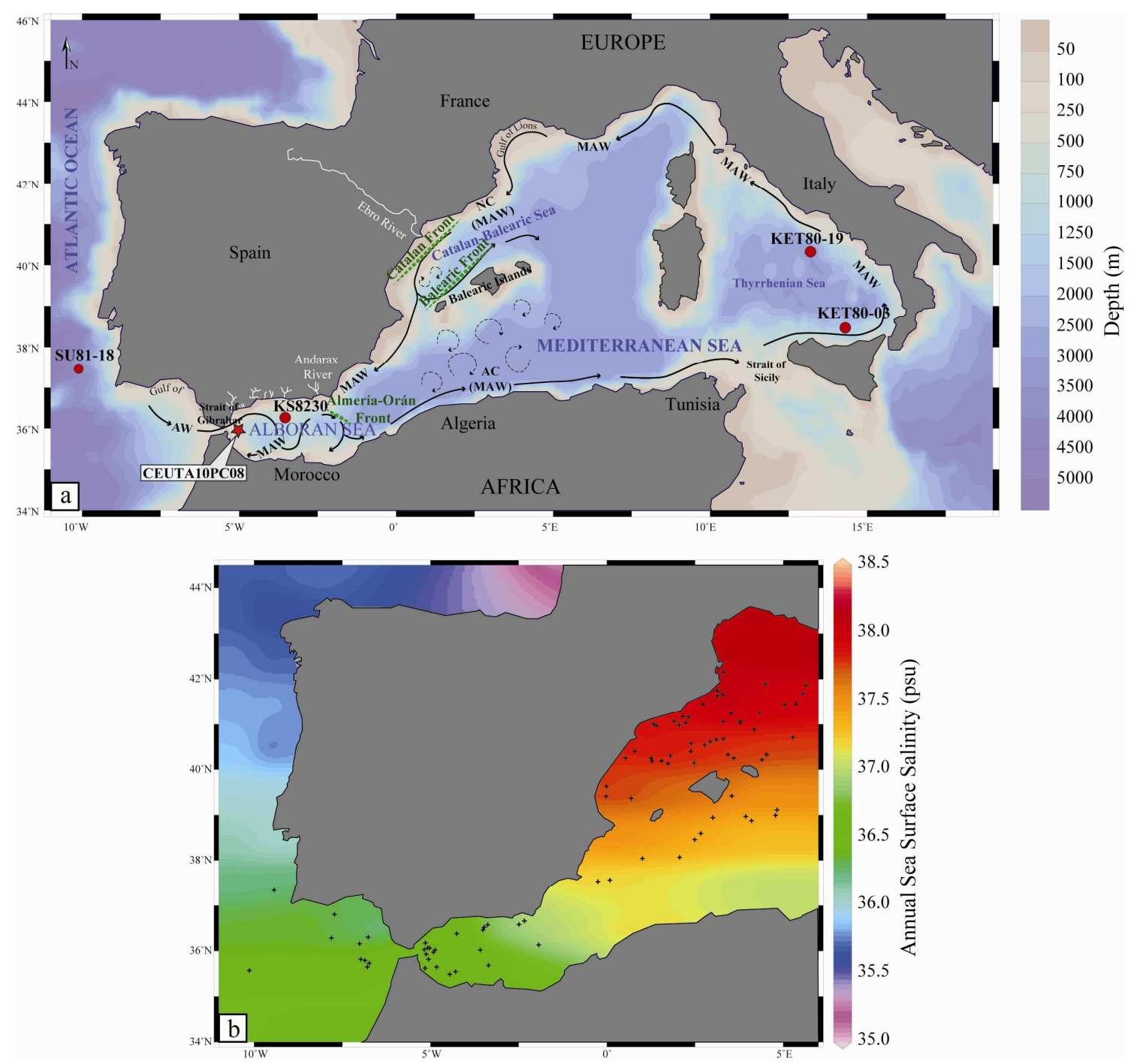

Figure 6.1. Maps of the Western Mediterranean Sea. a) Location of core CEUTA10PC08 in the Alboran Sea marked by a red star and other sediment cores in the Tyrrhenian Sea (KET80-03 and KET80-19; Kallel et al., (1997)), the Alboran Sea (KS8230; Emeis et al., (2000)) and the Atlantic Ocean (SU81-18; Duplessy et al., (1992)) used for validation of the reconstruction marked by red dots. Black arrows trace general surface circulation. Legend: AW: Atlantic Water. MAW: Modified Atlantic Water. NC: Northern Current. b) Location of the training set samples plotted over the annual mean SSS (psu) in the study area with Ocean Data View (Schlitzer, 2014). 


\section{3.-MATERIALS AND METHODS}

\subsection{1.-Modern training set}

\subsubsection{1.-Surface sediment samples}

Initially, 118 core tops located around a horizontal transect along the Western Mediterranean Sea and near the Gulf of Cadiz in the Atlantic Ocean (Fig. 6.1a) were selected. They had been retrieved at varying water depths ranging from 70 to $2620 \mathrm{~m}$ during several oceanographic surveys and were stored at the University of Vigo and at the Core Repository of the Institute of Marine Sciences- CSIC in Barcelona. The first $\mathrm{cm}$ (or the second, if the first was unavailable) of the 118 core tops was sampled, assuming that it essentially represented present-day conditions.

\subsubsection{2.-Environmental variables}

Data on temperature (Locarnini et al., 2013), salinity (Zweng et al., 2013), chlorophyll $a$ (Boyer et al., 2013), the oxygen content and saturation (García et al., 2014a), nitrate, phosphate, and silicate (García et al., 2014b) for the training set sites were taken from the 2013 World Ocean Atlas (WOA13) $1^{\circ}$ longitude by $1^{\circ}$ latitude grid, using weighted averaged gridding by Ocean Data View software (Schlitzer, 2014). These data have been averaged annually and seasonally (for summer and winter) from 1955 to 2012 and were selected at 10, 20, 30, 50, 75, $100,125,150,200$ and 300 meters water depth.

\subsection{2.-Fossil data set}

The fossil data set consisted of 297 samples taken systematically every $2 \mathrm{~cm}$ from core CEUTA10PC08, with a good-to-moderate degree of preservation. Coccolith census counts from this core previously published by Ausín et al. (2015) were used for the reconstruction. Core CEUTA10PC08 $\left(36^{\circ} 1^{\prime} 22^{\prime \prime} \mathrm{N}, 4^{\circ} 52^{\prime} 3^{\prime \prime} \mathrm{W}\right.$; $\left.914 \mathrm{mbsl}\right)$ was recovered from the Alboran Sea and its location lies under the modern path of the AW at the surface, near the Strait of Gibraltar (Fig. 6.1a). It consists mainly of calcareous oozes with silt. Sediment core chronostratigraphy was based on 15 radiocarbon ages and covered the time span from 25 to 4.5 ka calibrated BP at a $\sim 70$ yr temporal resolution (Ausín et al., 2015). All dates reported in this study are given in calibrated ages BP.

\subsection{3.-Micropaleontological analyses}

Both modern (surface data) and fossil (downcore) samples were prepared for coccolithophore analyses according to the techniques proposed by Flores and Sierro (1997). A Nikon Eclipse 80-i petrographic microscope with a phase contrast device at 1000x magnification was employed to identify and count at least 500 coccoliths in each sample, belonging to 21 different taxa. Species whose relative abundance was $<1 \%$ in the first count were considered later in 20 visual fields in order to estimate their abundance accurately. The 
final relative abundance of each species in each sample was then recalculated. Gephyrocapsa specimens smaller than $3 \mu \mathrm{m}$ were lumped together and designated "small Gephyrocapsa" (Flores et al., 1997). The "medium Gephyrocapsa" group was made up of Gephyrocapsa whose size was between 3 and $5 \mu \mathrm{m}$. The following species were split according to the size criteria: Emiliania huxleyi $(<4 \mu \mathrm{m}$ and $>4 \mu \mathrm{m})$ and Gephyrocapsa oceanica $(<5 \mu \mathrm{m}$ and $>5 \mu \mathrm{m})$. Other taxa identified in this study were Calcidiscus leptoporus, Florisphaera profunda, Gephyrocapsa caribbeanica, Gephyrocapsa muellerae, Helicosphaera spp., and Syracosphaera spp. (as dominant taxa). The rare taxa identified were Braarudosphaera bigelowii, Calciosolenia murrayi, Coccolithus pelagicus subsp. braarudii, Coccolithus pelagicus subsp. pelagicus, Discosphaera tubifera, Oolithotus fragilis, Pontosphaera spp., Rhabdosphaera clavigera, Umbilicosphaera spp. and Umbellosphaera spp.

Twenty-nine samples were finally eliminated from the initial modern data set owing to their high content in reworked nannofossils. These taxa belong to older stratigraphic levels (consistently older than the Pliocene in this study), meaning that they were resuspended and transported from their original location to the sample site. Hence, reworked nannofossils do not represent present-day conditions at the sample location and samples containing them in high quantities cannot be used to calibrate modern species-environment relationships.

The final training set (Table 6.1) comprised 89 surface samples: 78 from the Western Mediterranean and 10 from the Atlantic Ocean (Fig. 6.1b).

Table 6.1

Surface sample coordinates and environmental variables used in this study. Source: ${ }^{\text {a }}$ Core Repository of the Institute of Marines Sciences- CSIC of Barcelona and ${ }^{b}$ University of Vigo. *Outliers deleted from the final training set. SD: Standar Deviation units.

\begin{tabular}{|c|c|c|c|c|c|c|c|c|c|c|}
\hline Sample name & 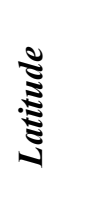 & 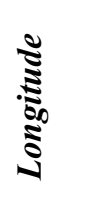 & $\tilde{\xi}$ & $\tilde{\xi}$ & 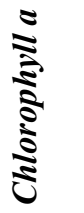 & 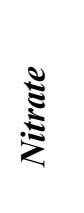 & $\frac{\mathfrak{s}}{\frac{5}{5}}$ & 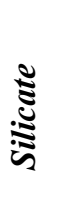 & 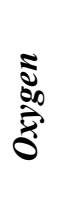 & 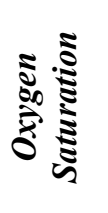 \\
\hline a STR-93/TG1 & 36.05 & -3.59 & 18.00 & 36.68 & 0.23 & 0.86 & 0.18 & 1.94 & 5.43 & 102.12 \\
\hline${ }^{* \mathrm{a}} \mathrm{VALSIS} / \mathrm{KF}-16$ & 41.25 & 3.53 & 17.35 & 38.00 & 0.10 & 0.99 & 0.14 & 1.25 & 5.39 & 99.63 \\
\hline${ }^{* \mathrm{a}} \mathrm{CO}-81-2 / \mathrm{TK}-2$ & 36.13 & -1.93 & 18.38 & 36.89 & 0.24 & 0.63 & 0.15 & 1.43 & 5.43 & 102.58 \\
\hline àMAY96/MTL-1 & 36.47 & -3.50 & 18.12 & 36.73 & 0.25 & 0.84 & 0.17 & 1.77 & 5.43 & 102.23 \\
\hline${ }^{\mathrm{a}} \mathrm{CO}-80-4 / \mathrm{K}-25$ & 38.60 & 2.68 & 19.02 & 37.38 & 0.10 & 0.71 & 0.12 & 1.36 & 5.30 & 101.53 \\
\hline${ }^{\mathrm{a} C G}-90-1 / \mathrm{TG}-5$ & 36.39 & -4.25 & 18.01 & 36.59 & 0.23 & 1.15 & 0.18 & 1.92 & 5.43 & 101.97 \\
\hline à STR93/TG-11 & 36.07 & -5.07 & 18.07 & 36.48 & 0.20 & 1.47 & 0.18 & 1.88 & 5.44 & 101.87 \\
\hline${ }^{\mathrm{a}} \mathrm{CO}-81-1 / \mathrm{K}-87$ & 41.06 & 3.31 & 17.65 & 37.93 & 0.08 & 0.90 & 0.14 & 1.16 & 5.39 & 100.09 \\
\hline
\end{tabular}




\begin{tabular}{|c|c|c|c|c|c|c|c|c|c|c|}
\hline${ }^{\mathrm{a}} \mathrm{GC}-82-1 / \mathrm{TR}-137$ & 41.01 & 1.34 & 18.06 & 37.88 & 0.08 & 0.65 & 0.13 & 0.96 & 5.46 & 101.60 \\
\hline${ }^{\mathrm{a}} \mathrm{CO}-83-1 / \mathrm{K}-29$ & 40.25 & 1.26 & 18.33 & 37.83 & 0.07 & 0.54 & 0.13 & 0.92 & 5.46 & 102.19 \\
\hline${ }^{\mathrm{a}} \mathrm{CO}-83-1 / \mathrm{K}-10$ & 40.18 & 1.29 & 18.38 & 37.81 & 0.07 & 0.52 & 0.12 & 0.93 & 5.45 & 102.22 \\
\hline${ }^{\mathrm{a}} \mathrm{CO}-81-1 / \mathrm{TK}-10$ & 40.31 & 1.81 & 18.27 & 37.83 & 0.07 & 0.59 & 0.13 & 0.92 & 5.45 & 101.90 \\
\hline${ }^{\mathrm{a}} \mathrm{GC}-84-6 / \mathrm{TR}-552$ & 41.64 & 3.14 & 17.11 & 38.06 & 0.12 & 1.06 & 0.14 & 1.30 & 5.40 & 99.37 \\
\hline${ }^{\mathrm{a}} \mathrm{GC}-84-6 / \mathrm{TR}-598$ & 41.75 & 3.14 & 17.09 & 38.07 & 0.12 & 1.06 & 0.14 & 1.30 & 5.40 & 99.29 \\
\hline a GC-84-6/TR-606 & 41.68 & 3.29 & 17.10 & 38.07 & 0.12 & 1.06 & 0.14 & 1.30 & 5.40 & 99.31 \\
\hline a⿳MAYC96/CD-2 & 36.04 & -5.17 & 18.09 & 36.47 & 0.19 & 1.51 & 0.18 & 1.87 & 5.44 & 101.86 \\
\hline${ }^{\mathrm{a}}$ VALSIS/KF-7 & 40.41 & 2.38 & 18.23 & 37.82 & 0.08 & 0.64 & 0.13 & 0.93 & 5.43 & 101.51 \\
\hline${ }^{\mathrm{a}} \mathrm{CO}-80-4 / \mathrm{K}-16$ & 38.07 & 2.07 & 19.02 & 37.28 & 0.10 & 0.79 & 0.13 & 1.38 & 5.30 & 101.54 \\
\hline${ }^{\mathrm{a}} \mathrm{CO}-80-4 / \mathrm{K}-37$ & 40.33 & 4.54 & 18.16 & 37.76 & 0.08 & 0.83 & 0.14 & 1.18 & 5.34 & 100.22 \\
\hline${ }^{\mathrm{a}} \mathrm{CO}-80-4 / \mathrm{K}-39$ & 40.33 & 3.44 & 18.20 & 37.78 & 0.09 & 0.74 & 0.13 & 1.02 & 5.38 & 100.78 \\
\hline${ }^{\mathrm{a}} \mathrm{CO}-80-4 / \mathrm{K}-10$ & 38.05 & 1.02 & 18.98 & 37.32 & 0.14 & 0.66 & 0.13 & 1.28 & 5.32 & 101.59 \\
\hline a80-CL/TK-33 & 39.63 & 0.00 & 18.74 & 37.77 & 0.11 & 0.31 & 0.10 & 0.95 & 5.43 & 103.01 \\
\hline${ }^{\mathrm{a}} \mathrm{CO}-80-4 / \mathrm{K}-4$ & 37.54 & -0.24 & 18.88 & 37.18 & 0.22 & 0.75 & 0.14 & 1.19 & 5.34 & 101.71 \\
\hline${ }^{\mathrm{a}} \mathrm{CO}-80-3 / \mathrm{K}-2$ & 39.42 & -0.02 & 18.77 & 37.76 & 0.12 & 0.30 & 0.10 & 0.96 & 5.42 & 103.00 \\
\hline${ }^{\mathrm{a}} \mathrm{CO}-80-4 / \mathrm{K}-33$ & 39.12 & 4.84 & 18.86 & 37.47 & 0.10 & 0.80 & 0.12 & 1.43 & 5.29 & 100.86 \\
\hline ªAYC-96/MTL-2 & 36.53 & -3.47 & 18.15 & 36.74 & 0.25 & 0.83 & 0.17 & 1.73 & 5.43 & 102.26 \\
\hline a'MAYC-96/MTL-18 & 36.58 & -3.38 & 18.19 & 36.77 & 0.25 & 0.80 & 0.17 & 1.67 & 5.44 & 102.31 \\
\hline${ }^{\mathrm{a}}$ MAYC-96/ALM-22 & 36.66 & -2.33 & 18.40 & 36.92 & 0.23 & 0.63 & 0.15 & 1.33 & 5.44 & 102.60 \\
\hline a'MAYC-96/ALM-19 & 36.59 & -2.48 & 18.38 & 36.90 & 0.24 & 0.64 & 0.15 & 1.38 & 5.44 & 102.57 \\
\hline ªAYC-96/GDR-4 & 36.18 & -5.14 & 18.11 & 36.45 & 0.20 & 1.57 & 0.18 & 1.82 & 5.43 & 101.80 \\
\hline${ }^{\mathrm{a}} \mathrm{GC}-93-1 / \mathrm{TC}-35$ & 41.07 & 1.92 & 17.91 & 37.90 & 0.08 & 0.74 & 0.13 & 1.02 & 5.44 & 101.08 \\
\hline a GC-88-1/TG-12 & 42.15 & 3.31 & 16.92 & 38.08 & 0.13 & 1.12 & 0.16 & 1.31 & 5.38 & 98.76 \\
\hline a'STR-93/TG-8 & 35.82 & -5.05 & 18.02 & 36.51 & 0.19 & 1.39 & 0.18 & 1.95 & 5.44 & 101.94 \\
\hline${ }^{\mathrm{a}}$ E-3D-78/35418 & 38.95 & 3.01 & 18.96 & 37.45 & 0.10 & 0.67 & 0.11 & 1.31 & 5.31 & 101.52 \\
\hline${ }^{\mathrm{a}} \mathrm{GC}-88-1 / \mathrm{TR}-70$ & 42.29 & 3.32 & 16.84 & 38.09 & 0.13 & 1.14 & 0.17 & 1.31 & 5.37 & 98.56 \\
\hline a $79 \mathrm{KEB} / \mathrm{K}-30$ & 40.41 & 0.79 & 18.30 & 37.84 & 0.09 & 0.53 & 0.13 & 0.92 & 5.47 & 102.25 \\
\hline a $80-C L / T K-61$ & 40.25 & 0.53 & 18.40 & 37.82 & 0.09 & 0.49 & 0.12 & 0.93 & 5.46 & 102.40 \\
\hline${ }^{\mathrm{a}} \mathrm{CL}-83-1 / \mathrm{K}-104$ & 41.18 & 2.15 & 17.72 & 37.93 & 0.08 & 0.82 & 0.14 & 1.09 & 5.43 & 100.62 \\
\hline${ }^{\mathrm{a}} \mathrm{E}-30-78 / 35411$ & 39.42 & 3.55 & 18.84 & 37.57 & 0.10 & 0.66 & 0.11 & 1.17 & 5.31 & 101.30 \\
\hline asTR93/TG-13 & 36.07 & -5.02 & 18.05 & 36.49 & 0.19 & 1.44 & 0.18 & 1.89 & 5.44 & 101.88 \\
\hline
\end{tabular}


CAPÍTULO 6: high-resolution sea surface salinity reconstruction for the last $25 \mathrm{kyr}$ in the Western Mediterranean from a coccolithophore-based transfer function

\begin{tabular}{|c|c|c|c|c|c|c|c|c|c|c|}
\hline${ }^{\mathrm{a}} \mathrm{CO}-80-4 / \mathrm{K}-30$ & 38.97 & 3.95 & 18.96 & 37.42 & 0.10 & 0.76 & 0.11 & 1.40 & 5.30 & 101.16 \\
\hline${ }^{\mathrm{b}}$ CEUTA/MC-1 & 35.93 & -5.12 & 18.06 & 36.49 & 0.19 & 1.46 & 0.18 & 1.90 & 5.44 & 101.90 \\
\hline${ }^{\mathrm{b}}$ CEUTA/MC-4 & 35.65 & -4.82 & 17.93 & 36.55 & 0.18 & 1.27 & 0.19 & 2.01 & 5.44 & 101.94 \\
\hline${ }^{\mathrm{b}}$ CEUTA/MC-8 & 36.02 & -4.87 & 18.00 & 36.52 & 0.19 & 1.36 & 0.18 & 1.94 & 5.44 & 101.90 \\
\hline${ }^{\mathrm{b}} \mathrm{CEUTA} / \mathrm{MC}-7$ & 35.97 & -4.91 & 18.01 & 36.52 & 0.19 & 1.37 & 0.18 & 1.94 & 5.44 & 101.91 \\
\hline${ }^{\mathrm{b}}$ CEUTA/BC-5 & 35.62 & -5.15 & 18.22 & 36.35 & 0.23 & 1.84 & 0.18 & 1.65 & 5.42 & 101.53 \\
\hline${ }^{\mathrm{b}} \mathrm{CEUTA} / \mathrm{MC}-2$ & 35.49 & -4.45 & 17.88 & 36.59 & 0.17 & 1.13 & 0.19 & 2.05 & 5.44 & 101.94 \\
\hline${ }^{\mathrm{b}} \mathrm{CADIZ10/BC}-4$ & 35.74 & -6.73 & 18.56 & 36.38 & 0.24 & 1.84 & 0.15 & 1.45 & 5.42 & 102.02 \\
\hline${ }^{\mathrm{b}}$ CADIZ10/MC-8 & 35.82 & -6.97 & 18.60 & 36.37 & 0.26 & 1.86 & 0.14 & 1.37 & 5.41 & 101.98 \\
\hline${ }^{\mathrm{b}} \mathrm{CADIZ10} / \mathrm{BC}-2$ & 35.80 & -6.85 & 18.58 & 36.37 & 0.25 & 1.85 & 0.15 & 1.41 & 5.42 & 102.00 \\
\hline 'CADIZ10/MC-6 & 36.30 & -6.76 & 18.42 & 36.27 & 0.28 & 2.11 & 0.16 & 1.40 & 5.41 & 101.43 \\
\hline${ }^{\mathrm{b}} \mathrm{CADIZ10/MC-1}$ & 35.65 & -6.78 & 18.58 & 36.38 & 0.24 & 1.82 & 0.15 & 1.43 & 5.42 & 102.06 \\
\hline${ }^{\mathrm{b}} \mathrm{IODP} / \mathrm{GC}-2 \mathrm{~A}$ & 36.29 & -7.81 & 18.53 & 36.34 & 0.34 & 1.89 & 0.13 & 1.18 & 5.41 & 101.79 \\
\hline${ }^{\mathrm{b}} \mathrm{IODP} / \mathrm{WI}-1 \mathrm{~B}$ & 37.34 & -9.42 & 17.97 & 36.24 & 0.29 & 1.34 & 0.14 & 1.06 & 5.52 & 102.56 \\
\hline 'bPL-SHACK4 & 35.57 & 10.13 & 18.82 & 36.42 & 0.35 & 1.09 & 0.10 & 1.03 & 5.41 & 102.53 \\
\hline${ }^{\mathrm{a}} \mathrm{GC} / 09 \mathrm{~A} / \mathrm{MC}$ & 36.81 & -7.72 & 18.44 & 36.31 & 0.34 & 1.94 & 0.14 & 1.19 & 5.42 & 101.68 \\
\hline${ }^{\mathrm{a}} \mathrm{CO}-83-2 / \mathrm{K}-16$ & 41.07 & 3.79 & 17.60 & 37.93 & 0.09 & 0.93 & 0.14 & 1.20 & 5.38 & 99.84 \\
\hline${ }^{\mathrm{a}} \mathrm{CO}-81-2 / \mathrm{TR}-62$ & 35.55 & -4.28 & 17.87 & 36.61 & 0.17 & 1.08 & 0.19 & 2.05 & 5.44 & 101.95 \\
\hline${ }^{\mathrm{a}} \mathrm{CO}-80-4 / \mathrm{K}-28$ & 38.88 & 4.11 & 18.98 & 37.38 & 0.10 & 0.79 & 0.12 & 1.47 & 5.29 & 101.14 \\
\hline${ }^{\mathrm{a}} \mathrm{CO}-81-1 / \mathrm{K}-66$ & 41.46 & 5.37 & 17.37 & 37.98 & 0.12 & 0.99 & 0.17 & 1.36 & 5.34 & 99.09 \\
\hline${ }^{\mathrm{a}} \mathrm{CO}-81-1 / \mathrm{K}-4$ & 40.19 & 1.55 & 18.37 & 37.81 & 0.07 & 0.55 & 0.12 & 0.94 & 5.45 & 102.08 \\
\hline${ }^{\mathrm{a}} \mathrm{CO}-81-1 / \mathrm{K}-69$ & 41.45 & 5.05 & 17.35 & 37.98 & 0.12 & 1.01 & 0.16 & 1.34 & 5.34 & 99.12 \\
\hline${ }^{\mathrm{a}} \mathrm{STR} 93 / \mathrm{TG}-21$ & 35.68 & -3.35 & 18.00 & 36.69 & 0.24 & 0.78 & 0.18 & 1.96 & 5.43 & 102.14 \\
\hline${ }^{\mathrm{a}} \mathrm{CO}-80-4 / \mathrm{K}-23$ & 38.46 & 2.49 & 19.02 & 37.36 & 0.10 & 0.71 & 0.12 & 1.36 & 5.31 & 101.55 \\
\hline${ }^{\mathrm{a}} \mathrm{CO}-83-2 / \mathrm{K}-28$ & 41.90 & 4.50 & 17.09 & 38.05 & 0.15 & 1.07 & 0.17 & 1.33 & 5.36 & 98.86 \\
\hline${ }^{* \mathrm{a}} \mathrm{GC}-88-3 / \mathrm{TG}-15$ & 36.16 & -7.00 & 18.48 & 36.30 & 0.29 & 2.02 & 0.15 & 1.35 & 5.41 & 101.62 \\
\hline${ }^{* \mathrm{a}} \mathrm{GC}-83-2 / \mathrm{TR}-440$ & 40.97 & 1.41 & 18.08 & 37.88 & 0.08 & 0.65 & 0.13 & 0.96 & 5.46 & 101.62 \\
\hline${ }^{\mathrm{a}} \mathrm{CO}-81-1 / \mathrm{K}-61$ & 41.69 & 5.57 & 17.29 & 38.00 & 0.13 & 0.99 & 0.18 & 1.39 & 5.33 & 98.96 \\
\hline${ }^{\mathrm{a}} \mathrm{CO}-83-2 / \mathrm{K}-17$ & 41.03 & 3.79 & 17.66 & 37.92 & 0.09 & 0.91 & 0.14 & 1.19 & 5.38 & 99.92 \\
\hline${ }^{\mathrm{a}}$ VALSIS/KF-2 & 39.37 & 0.69 & 18.77 & 37.73 & 0.11 & 0.34 & 0.11 & 0.98 & 5.41 & 102.85 \\
\hline${ }^{\mathrm{a}}$ VALSIS/KF-13 & 40.68 & 3.30 & 18.07 & 37.82 & 0.09 & 0.77 & 0.14 & 1.02 & 5.39 & 100.69 \\
\hline${ }^{\mathrm{a}} \mathrm{CO}-83-2 / \mathrm{K}-8$ & 40.66 & 3.11 & 18.10 & 37.82 & 0.09 & 0.74 & 0.14 & 0.99 & 5.40 & 100.85 \\
\hline
\end{tabular}




\begin{tabular}{|c|c|c|c|c|c|c|c|c|c|c|}
\hline${ }^{\mathrm{a}} \mathrm{CO}-83-2 / \mathrm{K}-49$ & 40.71 & 5.28 & 17.96 & 37.81 & 0.09 & 0.89 & 0.15 & 1.28 & 5.32 & 99.81 \\
\hline${ }^{\mathrm{a}}$ VALSIS/KF-5 & 40.16 & 2.48 & 18.36 & 37.78 & 0.09 & 0.62 & 0.13 & 0.96 & 5.41 & 101.56 \\
\hline${ }^{\mathrm{a}} \mathrm{CO}-81-17 / \mathrm{K}-60$ & 41.88 & 5.66 & 17.19 & 38.03 & 0.14 & 1.01 & 0.18 & 1.40 & 5.33 & 98.78 \\
\hline aGC-93-1/TG3 & 41.17 & 2.33 & 17.69 & 37.94 & 0.08 & 0.84 & 0.14 & 1.10 & 5.42 & 100.51 \\
\hline${ }^{\mathrm{a}} \mathrm{CO}-80-47 \mathrm{~K}-34$ & 40.22 & 4.41 & 18.24 & 37.74 & 0.09 & 0.81 & 0.14 & 1.17 & 5.33 & 100.35 \\
\hline${ }^{\mathrm{a}} \mathrm{E}-3 \mathrm{D}-78 / 35388$ & 39.00 & 4.78 & 18.90 & 37.43 & 0.10 & 0.81 & 0.12 & 1.48 & 5.29 & 100.94 \\
\hline${ }^{\mathrm{a}} \mathrm{CO}-80-4 / \mathrm{K}-38$ & 40.26 & 3.60 & 18.24 & 37.77 & 0.09 & 0.75 & 0.13 & 1.05 & 5.37 & 100.73 \\
\hline${ }^{\mathrm{a}}$ VALSIS/KF-17 & 41.28 & 4.33 & 17.39 & 37.98 & 0.11 & 1.00 & 0.15 & 1.29 & 5.37 & 99.39 \\
\hline${ }^{\mathrm{a}} \mathrm{GC}-93-1 / \mathrm{TC}-38$ & 40.99 & 2.06 & 17.97 & 37.88 & 0.08 & 0.72 & 0.13 & 1.00 & 5.44 & 101.15 \\
\hline${ }^{\mathrm{a}} \mathrm{CO}-83-2 / \mathrm{K}-7$ & 40.62 & 2.93 & 18.14 & 37.82 & 0.09 & 0.71 & 0.13 & 0.97 & 5.41 & 101.03 \\
\hline${ }^{\mathrm{a}}$ VALSIS/KF-12 & 41.03 & 2.24 & 17.88 & 37.90 & 0.08 & 0.77 & 0.14 & 1.04 & 5.43 & 100.90 \\
\hline a CO-80-4/K-9 & 37.57 & 0.09 & 18.91 & 37.18 & 0.21 & 0.77 & 0.14 & 1.19 & 5.33 & 101.63 \\
\hline a $\mathrm{CO}-83-2 / \mathrm{K}-6$ & 40.55 & 2.78 & 18.17 & 37.82 & 0.09 & 0.69 & 0.13 & 0.95 & 5.42 & 101.19 \\
\hline${ }^{\mathrm{a}} \mathrm{CO}-81-1 / \mathrm{K}-16$ & 40.14 & 1.74 & 18.40 & 37.79 & 0.08 & 0.55 & 0.12 & 0.95 & 5.43 & 102.02 \\
\hline${ }^{\mathrm{a}} \mathrm{CO}-81-1 / \mathrm{K}-74$ & 40.88 & 4.18 & 17.86 & 37.85 & 0.09 & 0.88 & 0.14 & 1.18 & 5.36 & 100.01 \\
\hline${ }^{\mathrm{a}}$ VALSIS/KF-9 & 40.58 & 2.39 & 18.18 & 37.83 & 0.08 & 0.65 & 0.13 & 0.93 & 5.44 & 101.46 \\
\hline & & $\begin{array}{l}\text { Mean } \\
\text { SD }\end{array}$ & 0.55 & 0.64 & 0.07 & 0.41 & 0.02 & 0.33 & 0.05 & 1.14 \\
\hline
\end{tabular}

\subsection{4.-Statistical analyses}

\subsubsection{1.-Relationship between coccolithophore assemblages and environmental variables}

Coccolithophore relative abundances were square-root transformed to stabilize their variances. None of the environmental variables under study showed a unimodal distribution. However, their $\log _{10}$ and square-root transformations did not cause noticeable changes, and consequently the variables were kept untransformed. The species Braarudosphaera sp., Discosphaera sp., and Calciosolenia sp., were excluded from the modern (and consequently from the fossil) coccolithophore assemblages since their maximum relative abundance was not $>1 \%$ in at least two samples.

Detrended Correspondence Analysis (DCA) was performed on the modern coccolithophore assemblage to estimate the length of the environmental gradient. A length of the first DCA axis $>2$ Standard Deviation (SD) units indicates the unimodal responses of the organisms (ter Braak and Prentice, 1988; Birks, 1995), while shorter lengths indicate linear responses. 
Canonical Correspondence Analysis (CCA) was used to evaluate the influence of the initial eight environmental variables to explain coccolithophore distribution in the modern training set.

The next step was to identify, by forward selection, the variables that explained the variance in the coccolithophore training set independently and to exclude strongly collinear variables. Because correlated variables entail redundant information, complicating the interpretation of the analyses, only significant variables at a p-value $<0.05$ were retained for subsequent analyses.

An individual CCA was also performed on the reduced environmental set to estimate the individual importance of each parameter in explaining the variability in the coccolithophore assemblage data. The ratio between the first constrained axis and the first unconstrained axis $\left(\lambda_{1} / \lambda_{2}\right)$ was calculated and used as an initial diagnosis to test the strength of a single environmental variable when the effects of those remaining were excluded from the analyses (ter Braak and Juggins, 1993). If $\lambda_{1} / \lambda_{2} \geq 1$, the variable under study is considered to be important one for explaining the distribution of the species.

The proportion of the variance in the coccolithophore training set explained uniquely by each significant environmental variable was calculated through variance partitioning.

Ordinations were performed using PAST (Hammer et al., 2001) and R software (R Development Core Team, 2011) with the add-on VEGAN package (Oksanen et al., 2007).

\subsubsection{2.-Transfer function}

The weighted average-partial least squares (WA-PLS) (ter Braak and Juggins, 1993; ter Braak et al., 1993) and the Modern Analog Technique (MAT) (Prell, 1985) were performed to test the calibration and regression models implemented with the C2 software (Juggins, 2007). All models were calculated for the cross-validation predictions by bootstrapping (999 permutation cycles) (Birks, 1995). In MAT, the number of analogs resulting in the maximum coefficient of determination $\left(\mathrm{R}_{\text {boot }}^{2}\right)$ between the observed and predicted values and the lowest root-mean square error of prediction (RMSEP) (Telford et al., 2004) was calculated using an optimization set together with the usual training and test sets implemented in the analoge package for R (R Development Core Team, 2007). In WA-PLS, a decrease of $5 \%$ or more in RMSEP was required to retain the next component (ter Braak et al., 1993; Birks, 1995). Thus, the residual structure in the data set was used to improve the prediction (ter Braak and Juggins, 1993).

Outliers may reduce the power of prediction of the calibration model as well as introducing undesirable effects in model coefficients (Birks, 1995). Potential outliers, determined as those whose absolute residual was higher than the mean SD of the observed values (Edwards et al., 2004), were excluded from the final models. 
Telford et al. (2013) reported that SST reconstructions based on planktonic foraminifera census counts calibrated at a fixed depth and for a particular season might be biased. The most suitable calibration should be based on the depth and season that most influenced the coccolithophore fossil assemblage from core CEUTA10PC08. These were determined by testing the statistical significance of the summer, winter, and annual reconstructions of the variable of interest at ten different depths of the upper photic zone from 10 to $125 \mathrm{~m}$. We followed the procedure described by Telford et al. (2013), using the palaeoSig package v.1.1-1 (Telford, 2012) for R (R Development Core Team, 2011).

A combination of the highest $\mathrm{R}_{\text {boot }}^{2}$ coefficient, the lowest maximum bias (Max_Bias ${ }_{\text {boot }}$ ), and the lowest RMSEP was used as a criterion for the quality prediction of the model. The graphical representation of the observed values against the values predicted by the model and the residuals against the predicted values were used as a diagnosis of the model.

\subsubsection{3.-SSS reconstruction and evaluation}

MAT and WA-PLS were applied to the fossil coccolithophore assemblages of core CEUTA10PC08, which were previously square-root transformed.

In order to assess the quality of the modern analogs for the fossil (downcore) samples, the squared chord distance between each fossil sample and each sample in the modern training set (Overpeck et al., 1985) was calculated with MAT using program C2 version 1.4.3 (Juggins, 2007). A good analog was defined as a fossil sample having a squared chord distance of less than the $5^{\text {th }}$ percentile of the distribution of all distances between modern samples in the training set (Simpson, 2007). A squared chord distance below the $10^{\text {th }}$ percentile would be considered good, while values above this cutoff would represent poor analog assemblages.

In order to test whether the reconstruction represented the most important variable for coccolithophore assemblages in the past, an ordination was carried out on the fossil coccolithophore assemblages. After Hellinger transformation of the data (Rao, 1995), Principal Component Analyses (PCA) were performed on the fossil assemblage dataset. PC1 summarizes the maximum variance in the fossil dataset. The reconstructed SSS was compared with the scores of PC1 to assess whether the reconstruction could be considered representative based on the major ecological changes of the fossil assemblage as shown in the ordination (Juggins, 2013).

Validation of the reconstruction obtained was carried out by comparing it with other published reconstructions based on independent proxies from the study area. 


\section{4.-RESULTS}

\subsection{1.-Geographical distribution of coccolithophores}
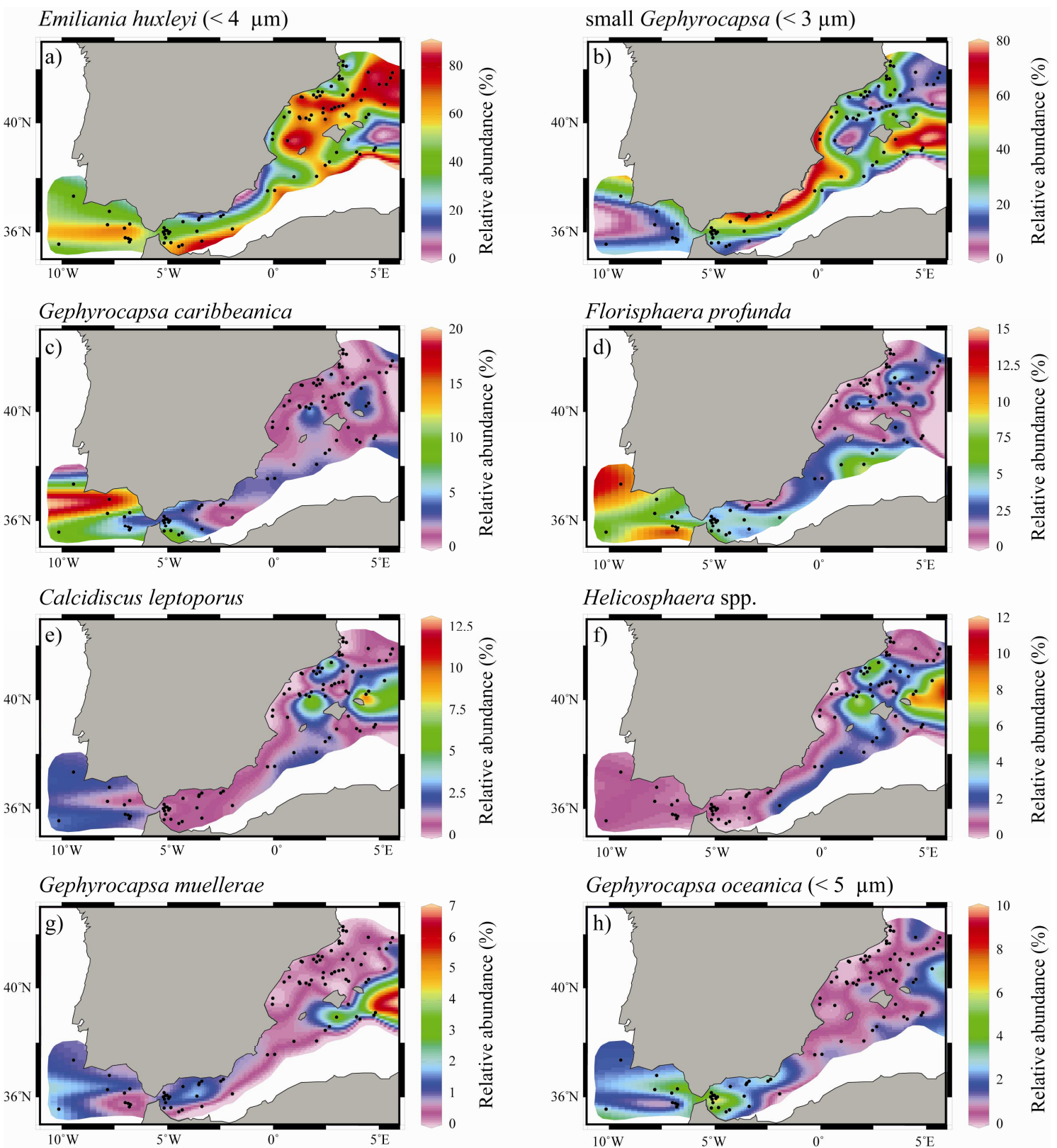

Figure 6.2. Distribution of the main coccolithophore taxa considered in the training set studied according to their relative abundance (\%) in the Western Mediterranean Sea and Atlantic Ocean, Gulf of Cadiz: a) Emiliania huxleyi $(<4 \mu \mathrm{m})$. b) small Gephyrocapsa $(<3 \mu \mathrm{m}) \mathrm{c})$. Gephyrocapsa caribbeanica. d) Florisphaera profunda. e) Calcidiscus leptoporus. f) Helicosphaera spp. g) Gephyrocapsa muellerae. h) Gephyrocapsa oceanica $(<5 \mu \mathrm{m})$.

The geographical distribution of the main coccolithophore taxa is shown in figure 2. This figure illustrates that the relative abundance of $E$. huxleyi $<4 \mu \mathrm{m}$ increases from the southern Spanish coast to the northern African coast, and high values are observed around the mouth of the Ebro River Delta (Fig. 6.2a). By contrast, the small Gephyrocapsa group (Fig. 6.2b) shows 
the opposite distribution: a decreasing relative abundance from the Spanish to the African coast and low values near the Ebro River Delta. E. huxleyi $<4 \mu \mathrm{m}$ and the small Gephyrocapsa group are small placoliths that on average constitute $83 \%$ of coccolithophore assemblages. $G$. caribbeanica and F. profunda (Fig. 6.2c, d) are more abundant in the Atlantic Ocean (up to 16 $\%$ and $13 \%$, respectively). C. leptoporus and Helicosphaera spp. (Fig. 6.2e, f) show patchy distributions and are more abundant (up to $7 \%$ ) around the Catalan margin and the Balearic Islands. G. muellerae (Fig. 6.2g) is more abundant southeast of the Balearic Islands and shows a patch of $2 \%$ abundance in the northern part of the Alboran Sea. G. oceanica $(<5 \mu \mathrm{m})$ (Fig. $6.2 \mathrm{~h}$ ) is mostly distributed near the Strait of Gibraltar, reaching up to $6 \%$ of relative abundance. It also shows a patch of $3 \%$ relative abundance near the Almería-Orán Front and around the Andarax River mouth (Figs. 1a, 2h).

\subsection{2.-Relationship between coccolithophores and environmental variables}

The first axis of the DCA performed on the modern coccolithophore assemblage was 2.6 SD units. Accordingly, unimodal methods were followed. The CCA (Fig. 6.3) reveals species distribution along the eight initial environmental variables (temperature, salinity, chlorophyll $a$, oxygen content and saturation, nitrate, phosphate, and silicate). Salinity exhibits the longest gradient and is more strongly correlated with axis 1, which explains $65.1 \%$ of the total variance in the data. Axis 2 is much weaker and explains $19.9 \%$ of the total variation.

Forward selection revealed that only salinity, nitrate, and silicate afford unique information, explaining variation in the training set alone, and they are significant at the $95 \%$ level when added individually to the model. Individual CCAs give $\lambda_{1} / \lambda_{2}=1.43$ for salinity, $\lambda_{1} / \lambda_{2}=0.81$ for nitrate, and $\lambda_{1} / \lambda_{2}=0.65$ for silicate, showing that salinity is the most important variable among those found to be significant. Variance partitioning revealed that these significant variables together explain $32.5 \%$ of the total variance in the coccolithophore training set and salinity explains almost half of this variance $(15.5 \%)$.

\subsection{3.-Transfer functions}

SSS was chosen to develop the coccolithophore-based transfer function because it explained the largest amount of variation in the coccolithophore assemblages. Among the WA-PLS models, the two-component model (WA-PLS2) was chosen as the most suitable since it afforded a reduction of $7 \%$ in the RMSEP. The ideal number of analogs for MAT was six.

Four samples (Table 6.1) were identified as potential outliers in preliminary models developed with both MAT and WA-PLS. Their removal from subsequent model implementations led to an improvement of the $\mathrm{R}_{\text {boot }}^{2}$ coefficient of $7.2 \%$ and $8 \%$, respectively, and reduced both Max_Bias boot $_{\text {and }}$ RMSEP. 
The analyses of the amount of down-core variance explained by the summer, winter, and annual SSS reconstructions at ten different depths and their statistical significance revealed that the mean-annual reconstruction at $10 \mathrm{~m}$ explained the highest variance. Hence, the SSS reconstruction for core CEUTA10PC08 was based on the mean-annual salinity data at $10 \mathrm{~m}$ depth.

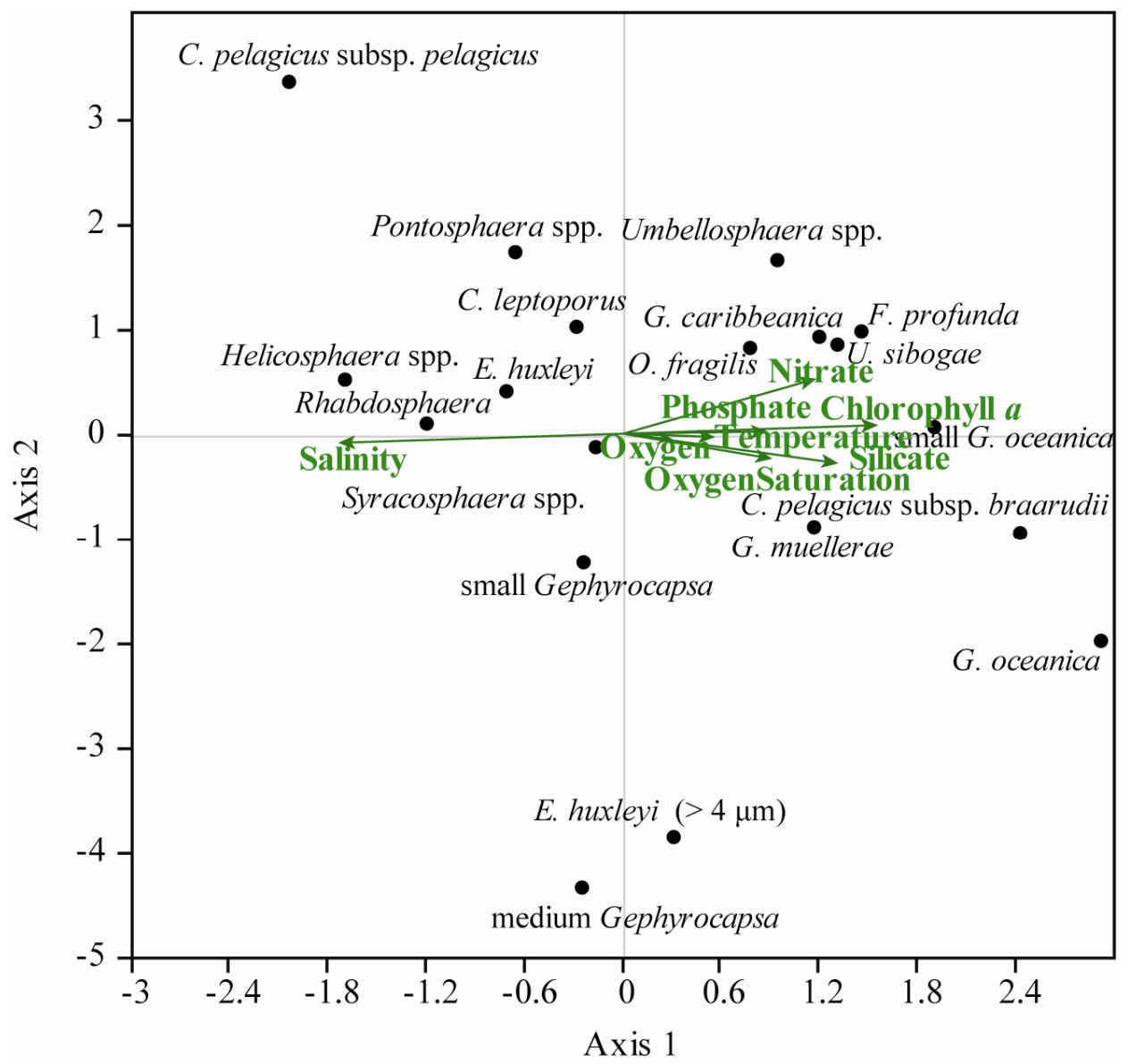

Figure 6.3. CCA plot based on the 19 taxa (black dots) and eight environmental variables (green arrows): temperature, salinity, chlorophyll $a$, oxygen content, oxygen saturation, nitrate, phosphate, and silicate from the 89 surface sample sites.

MAT and WA-PLS2 boot-strapped coefficients are shown in table 6.2. Both methods showed similar quality predictions, although MAT was seen to perform slightly better than WAPLS2 from a higher $\mathrm{R}_{\text {boot }}^{2}$ and a lower RMSEP. The predicted versus observed values from both models are illustrated in figures $4 \mathrm{a}, \mathrm{b}$. All points approach the diagonal of slope one reasonably well, which indicates perfect predictions. The salinity gradient ranges from 36.2 to $38.2 \mathrm{psu}$. Intermediate values (37.1- 37.6 psu) are less well represented by the observations. The residuals for MAT and WA-PLS models (Fig. 6.4c, d) are distributed around zero and show no apparent patterns. 

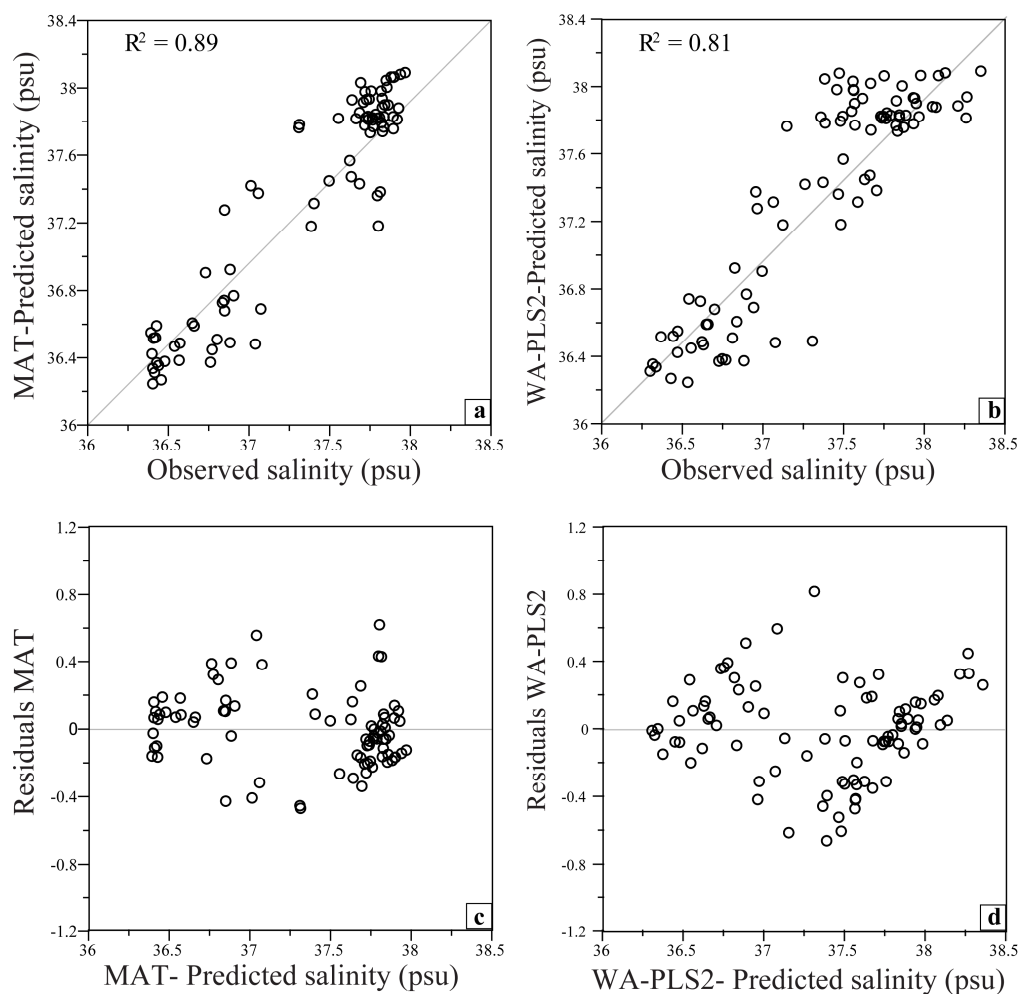

Figure 6.4. Diagnostic graphs of the models: a) Observed $v s$ MAT-predicted salinity values. b) Observed vs WA-PLS-predicted salinity values. c) MAT-predicted salinity values vs residuals. d) WA-PLS2predicted salinity values $v s$ residuals.

Table 6.2

Model coefficients from MAT and WA-PLS2 cross-validated by boot-strapping. $\mathrm{R}_{\text {boot }}^{2}$ : bootstrapped coefficient of determination between the observed and predicted values. Max_Bias boot: $_{\text {: bootstrapped }}$ maximum bias. RMSEP: root mean square error of prediction (psu).

\begin{tabular}{c|cc}
\hline & MAT & WA-PLS2 \\
\hline $\mathbf{R}_{\text {boot }}^{2}$ & 0.887 & 0.813 \\
Max_Bias $_{\text {boot }}$ & 0.195 & 0.182 \\
RMSEP & 0.257 & 0.287 \\
\hline
\end{tabular}

\subsection{4.-SSS reconstruction}

The SSS reconstruction derived from both MAT and WA-PLS are very similar (Fig. 6.5a, b), with same range of SSS values, and only differ in the trend during the stadials associated with Heinrich Events 2 and 1 (H2 and H1), when the WA-PLS-estimated SSS shows more pronounced salinity decreases. 

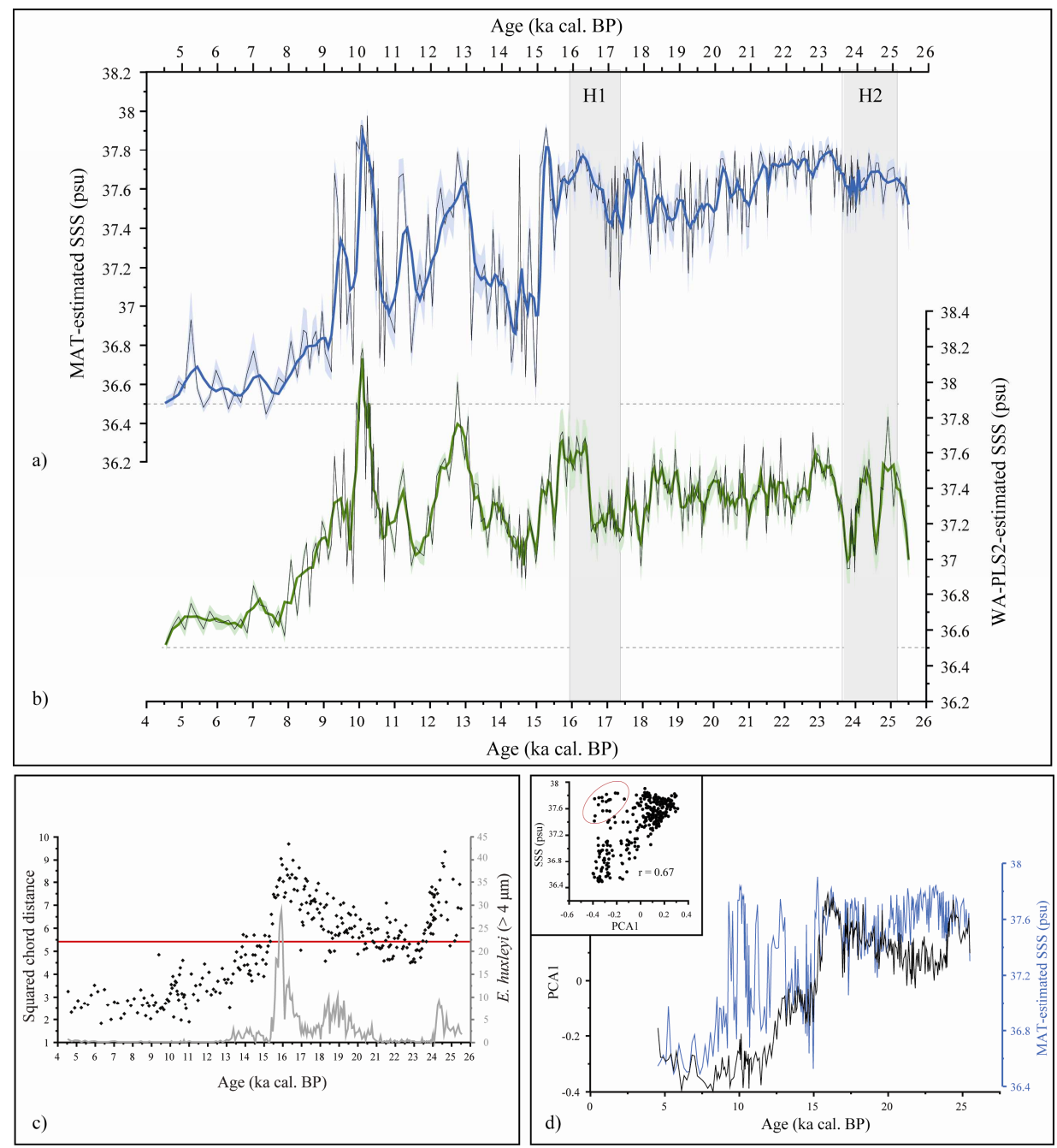

Figure 6.5. SSS reconstructions for core CEUTA10PC 08 derived from a) MAT and b) WA-PLS2. In both graphs, the thin black lines represent the estimated values while the thick blue/green lines represent these original data fitted to a 3-point moving average smoothing spline. Pale blue/green shadows represent the error range, and dashed lines indicate current annual mean SSS in the Alboran Sea from the World Ocean Atlas 2013 (WOA13) (Zweng et al., 2013). Grey bars delimit the stadials associated with Heinrich events 2 and 1 (H2 and H1). c) Dissimilarity between modern and fossil assemblages (black dots) measured by squared chord distance (left axis) plotted $v s$ age. The red line indicates the $10^{\text {th }}$ percentile (no analog). Relative abundance of the species E. huxleyi ( $>4 \mu \mathrm{m})(\%$; right axis). d) Scatter plot (top left corner) and profiles comparing the PC1 (black line) and MAT-estimated SSS (blue line). The red circle contains the 17 samples that deviate from the general good fit.

The SSS reconstructions obtained from core CEUTA10PC08 (Fig. 6.5a) can be divided into three intervals: i) the period from 25.5 to $15.5 \mathrm{ka}$ is characterized by higher values, ranging between 37.9 and $37 \mathrm{psu}$; ii) the period from 15.5 to 9 ka shows fast changes and large oscillations within a range of $1.5 \mathrm{psu}$; and iii) the period from 9 to $4.5 \mathrm{ka}$ records the lowest values, ranging between 36.5 and 37 psu. In greater detail, lower values are found from 20 to 18 ka followed by a drop of $0.8 \mathrm{psu}$ at $17.3 \mathrm{ka}$ (Fig. 6.5a). An abrupt decrease from 37.9 to 36.5 psu is recognized at $15 \mathrm{ka}$, followed by large peaks of high values at 12.8, 11.1, and $10.2 \mathrm{ka}$. 
During the last interval ( 9 to $4.5 \mathrm{ka}$ ), the SSS shows a decreasing trend interrupted by two peaks at 7 and $5.2 \mathrm{ka}$.

The errors associated with SSS reconstruction are of a similar magnitude for the last $25 \mathrm{kyr}$ (Fig. 6.5a). Squared chord distances between fossil and modern assemblages (Fig. 6.5b) revealed that many samples from the first interval ( 25.5 to $15 \mathrm{ka}$ ) were above the $10^{\text {th }}$ percentile, especially those around 25 and $16 \mathrm{ka}$. The length of DC1 from fossil coccolithophore assemblages was 1.26 SD, indicating a linear response (Birks, 1995). The PC1 from fossil data is shown in figure 5c. Its comparison with the SSS reconstruction shows good agreement. The scatter plot of these shows that both data are highly correlated, with an $r=0.67$, although a set of 17 of the total 297 fossil samples deviate from this general good fit (Fig. 6.5c).

\section{5.-DISCUSSION}

\subsection{1.-Geographic coccolithophore distribution and SSS}

The geographic coccolithophore distribution and SSS reveal that E. huxleyi $(<4 \mu \mathrm{m})$ and small Gephyrocapsa $(<3 \mu \mathrm{m})$ are very abundant $(83 \%$ on average) and widespread in the Western Mediterranean (Fig. 6.2a, b). Comparable abundances of small placoliths have already been reported for surface sediment samples in the Western Mediterranean by Álvarez et al. (2010). These taxa, especially E. huxleyi $(<4 \mu \mathrm{m})$, are cosmopolitan and tolerate wide ranges of temperature and salinity (Winter and Siesser, 1994). The higher relative abundance of $G$. caribbeanica, F. profunda and G. oceanica in the Atlantic Ocean and the Mediterranean waters near the entrance of the Strait of Gibraltar (Fig. 6.2c, d, h) suggests their preference for fresher waters. This notion is also indicated by the CCA (Fig. 6.3), where these species are located on the right part of the salinity gradient. C. leptoporus (Fig. 6.2e) and Helicosphaera spp. (Fig. 6.2f) show higher abundances in the Catalan-Balearic Sea and a similar spatial distribution. Nevertheless, the CCA (Fig. 6.3) suggests that Helicosphaera spp. prefer for more saline waters than C. leptoporus. This discrepancy might be related to the patchy spatial distribution of these species, possibly linked to the influence of common mesoscale structures in this area such as eddies or geostrophic fronts (Fig. 6.1a).

Multivariate analyses revealed that salinity explains a significant proportion of the variance in the coccolithophore data, regardless of other environmental gradients. Moreover, salinity proved to be the most important variable of those studied in explaining coccolithophore data variance in the modern training set. These results on the coccolithophore-salinity relationship might have been influenced by the effect of seasonality in the Western Mediterranean Sea. The plotted summer and winter mean values of SST, nutrients, oxygen, and chlorophyll $a$ from the WOA13 show strong seasonal variability, while those of SSS remain constant along the year (Locarnini et al., 2013). Because SSS is not subject to noticeable seasonality, its effect may be 
recorded in an equivalent manner by both the environmental and the biological training data sets, SSS being the most important variable in explaining coccolithophore distribution in the surface sediments of the Western Mediterranean.

The link between coccolithophore assemblages and environmental parameters, such as productivity and temperature, has been observed in some studies performed in the Indian and Pacific oceans (Beaufort et al., 1997; Saavedra-Pellitero et al., 2011). However, the relationship between coccolithophores and salinity is more uncertain, since salinity does not seem to have been reported previously as an explanatory variable for coccolithophore distribution. Nevertheless, this notion is ecologically plausible since salinity has proved to be important to other marine planktonic groups such as diatoms (Li et al., 2012; Jiang et al., 2014) and dinoflagellate cysts (Jansson et al., 2014, and references therein). Moreover, some studies have reported the importance of salinity variations in relation to certain coccolithophore species. $E$. huxleyi is the most abundant and ubiquitous extant coccolithophore (Cros and Fortuño, 2002). While Paasche et al. (1996), Green et al. (1998), Schouten et al. (2006), Fielding et al. (2009), Bollmann and Herrle, (2007) and Bollmann et al. (2009) failed to find any significant correlation between coccolithophores and SST, they did find a direct relationship between varying salinities and the morphology of $E$. huxleyi in both culture experiments and marine surface sediment samples.

In the Japan Sea, salinity has been proposed to have an ecological or physiological influence on the production of alkenone and alkenoates, which are organic compounds mainly produced by Emiliania and Gephyrocapsa genus (Fujine et al., 2006). In the Baltic Sea, alkenone unsaturation ratios have been found to be significantly correlated with salinity (Blanz et al., 2005). In the Mediterranean Sea, Knappertsbusch (1993) studied the distribution of extant coccolithophore species in relation to in situ temperature and salinity data. A good correspondence was only found between coccolithophore species and the environmental parameters under study, indicating that G. oceanica was linearly correlated with salinity.

\subsection{2.-Transfer function quality}

MAT afforded a slightly higher $\mathrm{R}_{\text {boot }}$ and lower RMSEP than WA-PLS2 (Table 6.2). A general good fit can be deduced for both models from the high correlation coefficients between the observed and predicted values (Fig. 6.4a, b). The intermediate values of the salinity gradient are less well represented than the more extreme values. According to Telford and Birks (2011), if the observations are very unevenly distributed along the environmental gradient the RMSEP may be biased, overestimating the predictive power of the model. While an even distribution would be always desirable, unevenness is a feature inherent to most training sets from oceanic environments. In this case, the unevenness is not severe and the observations are distributed 
along the gradient, leaving no apparent gaps. The distribution of the residuals (Fig. 6.4c, d) indicates the adequacy of the model.

\subsection{3.-Downcore SSS reconstruction}

The performance of MAT and WA-PLS2 (Table 6.2) and their derived SSS reconstructions (Fig. 6.5a, b) are very similar. Nevertheless, WA-PLS2 afforded more marked salinity decreases than MAT during the stadials associated with H2 (25.2-23.7 ka) and H1 (17.4-15.9 ka). Unlike WA-PLS, MAT uses the local rather than the global fit between the species and the environment and is strongly dependent upon on the analogs selected (Telford and Birks, 2009). Fossil samples lack good analogs for the $\mathrm{H} 2$ and $\mathrm{H} 1$ (Fig. 6.5 c), coinciding with large peaks of $E$. huxleyi $(>4 \mu \mathrm{m})$ (Fig. 6.5c), as previously shown in the Alboran Sea by Colmenero et al. (2004). H2 and H1 have been linked to the entry of fresher water originating from the North Atlantic ice melting in the Western Mediterranean Sea (Cacho et al., 1999; Sierro et al., 2005; Melki, 2011), suggesting a link between the high abundances of E. huxleyi $(>4 \mu \mathrm{m})$ and lower salinities in the past. By contrast, Bollmann and Herrle, (2007) reported a significant current correlation between E. huxleyi $(>4 \mu \mathrm{m})$ and higher salinities from the study of globally distributed core-top samples. Bollmann and Herrle, (2007) used this relationship to estimate salinity values during the LGM, but observed several overestimations with regard to other published values. Interestingly, those samples were characterized by high relative abundances of E. huxleyi $(>4 \mu \mathrm{m})$. These discrepancies suggest that E. huxleyi $(>4 \mu \mathrm{m})$ in ancient sediments lacks an analog in modern assemblages, as indicated by the high squared chord distances of the fossil samples with high percentages of this species (Fig. 6.5c). These samples add uncertainty to the reconstruction, and must be taken with caution when interpreting reconstructed values in $\mathrm{H} 2$ and H1and part of the LGM.

Transfer functions assume that the ecological response of organisms to either the environmental variable of interest or to the linear combination of this important variable with others has not changed significantly over the time span represented by the fossil assemblage (Birks, 1995). PC1 from the fossil data shows the most important changes in the composition of the fossil coccolithophore assemblage. The similarity of PC1 and reconstructed SSS patterns (Fig. 6.5d) demonstrates that the relationship between coccolithophore assemblages and SSS fulfills this assumption back to $25 \mathrm{kyr}$. No explanation has been found for the 17 samples that deviate from the good general fit (Fig. 6.5d), and hence these were retained in the fossil data set. 


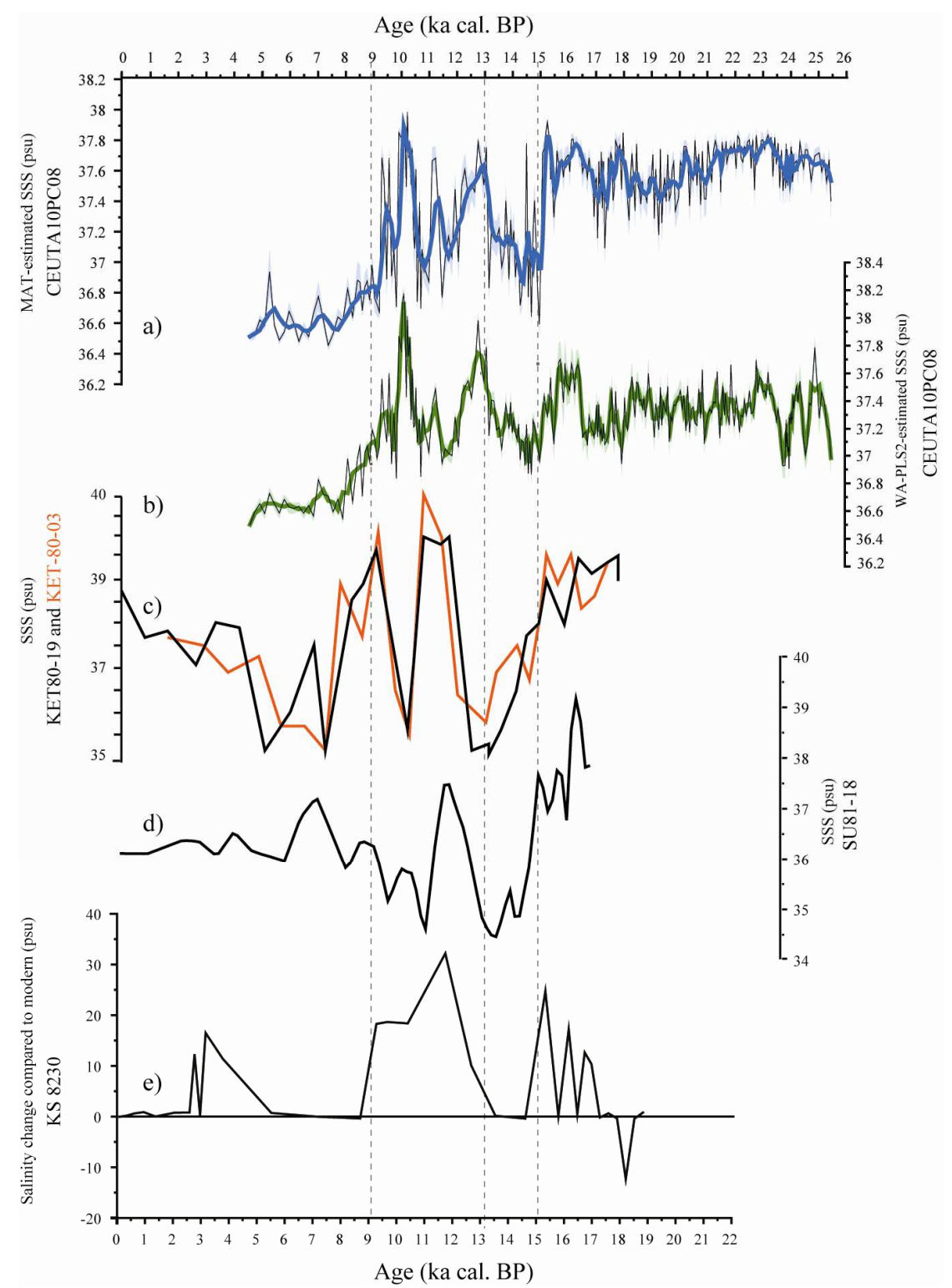

Figure 6.6. Comparison of sea surface salinity (SSS; in psu) reconstructions for core CEUTA10PC08 in the Alboran Sea derived from a) MAT and b) WA-PLS2; c) cores KET80-19 (black line) and KET80-03 (orange line) in the Tyrrhenian Sea (Kallel et al., 1997); d) core SU81-18 in the Atlantic Ocean (Duplessy et al., 1992); and e) salinity change compared to modern values from core KS 8230 in the Alboran Sea (Emeis et al., 2000).

\subsection{4.-SSS changes in the Alboran Sea over the last 25,000 yr}

SSS variability in the Alboran Sea over the last 25,000 yr in core CEUTA10PC08 shows similar patterns to those from nearby records (Duplessy et al., 1992; Kallel et al., 1997; Emeis et al., 2000) (Figs.1a, 6) estimated from oxygen isotope $\left(\delta^{18} \mathrm{O}\right)$ and SST data. MAT- and WAPLS2-estimated SSS reconstructions for core CEUTA10PC08 are most similar to that of its nearest core, KS 8230 (Figs.1a, 6a, b, e). All the profiles show salinity decreases during the 15- 
$13 \mathrm{ka}$ and 9-5 ka intervals. From 13 to $9 \mathrm{ka}$, the records show higher values affected by shortterm oscillations of high amplitude, not recorded in core KS8230 (Emeis et al., 2000) possibly owing to its lower resolution. The long-term variability of all the records is in good agreement with this, pointing to the reliability of the SSS reconstructions from core CEUTA10PC08 for the last $18 \mathrm{ka}$ (Fig. 6.6a, b).

\subsubsection{1.- $H 2$ and $H 1$}

From 25 to $18 \mathrm{ka}$ there are no records available for comparison. The MAT-SSS reconstruction (Fig. 6.6a) might involve additional uncertainty during $\mathrm{H} 2$ and $\mathrm{H} 1$, derived from the situation of the absence of analogs discussed above. Unlike what would be expected from the injection of fresher AW into the Alboran Sea (Cacho et al., 1999; Sierro et al., 2005), only slight decreases in salinity are seen at the end of $\mathrm{H} 2$ and at the beginning of $\mathrm{H} 1$. By contrast, WA-PLS2-estimated SSS (Fig. 6.6b) show more marked salinity decreases, being more coherent with a freshwater inflow scenario. Moreover, the Max_Bias boot is slightly higher for MAT (Table 6.2). This coefficient provides information about the tendency of the transfer function to over- or under-estimate the predicted values somewhere along the gradient (ter Braak and Juggins, 1993). Based on these observations, it seems that WA-PLS2 affords more reliable values than MAT for $\mathrm{H} 2$ and $\mathrm{H} 1$ (Fig. 6.7a).

\subsubsection{2.-Last Glacial Maximum (LGM)}

The period from 23.5 to 21 ka covers part of the LGM (23-19 ka), showing high SSS values (Fig. 6.7a). Global sea-level was estimated $120 \mathrm{~m}$ lower compared with present levels (Fairbanks, 1989), partially restricting the connection between the Mediterranean Sea and the Atlantic Ocean. Consequently, the reduced water exchange resulted in an increase in salinity during this interval.

\subsubsection{3.-Oldest Dryas}

An abrupt decrease in salinity of 0.8 psu occurred at $15.5 \mathrm{ka}$. This change is not supported by the findings of Fletcher and Sánchez Goñi (2008) or those of Combourieu Nebout et al. (2009) who, using pollen records from two sites in the Western Mediterranean, identified arid conditions in the southern Iberian Peninsula. The global sea-level rise of $\sim 20 \mathrm{~m}$ during meltwater pulse 1A (mwp-1A) has been dated at 14.6 ka (Bard et al., 1996; Weaver et al., 2003), simultaneous to the onset of the Bølling-Allerød Since this section covers 3,000 yr with no control point (Fig. 6.7), it could be an artifact of poorly constrained chronology for this time interval. Nevertheless, this seems unlikely since other authors (Duplessy et al., 1992; Kallel et al., 1997; Emeis et al., 2000) have reported similar abrupt SSS decreases at this time (Fig. 6.6). 


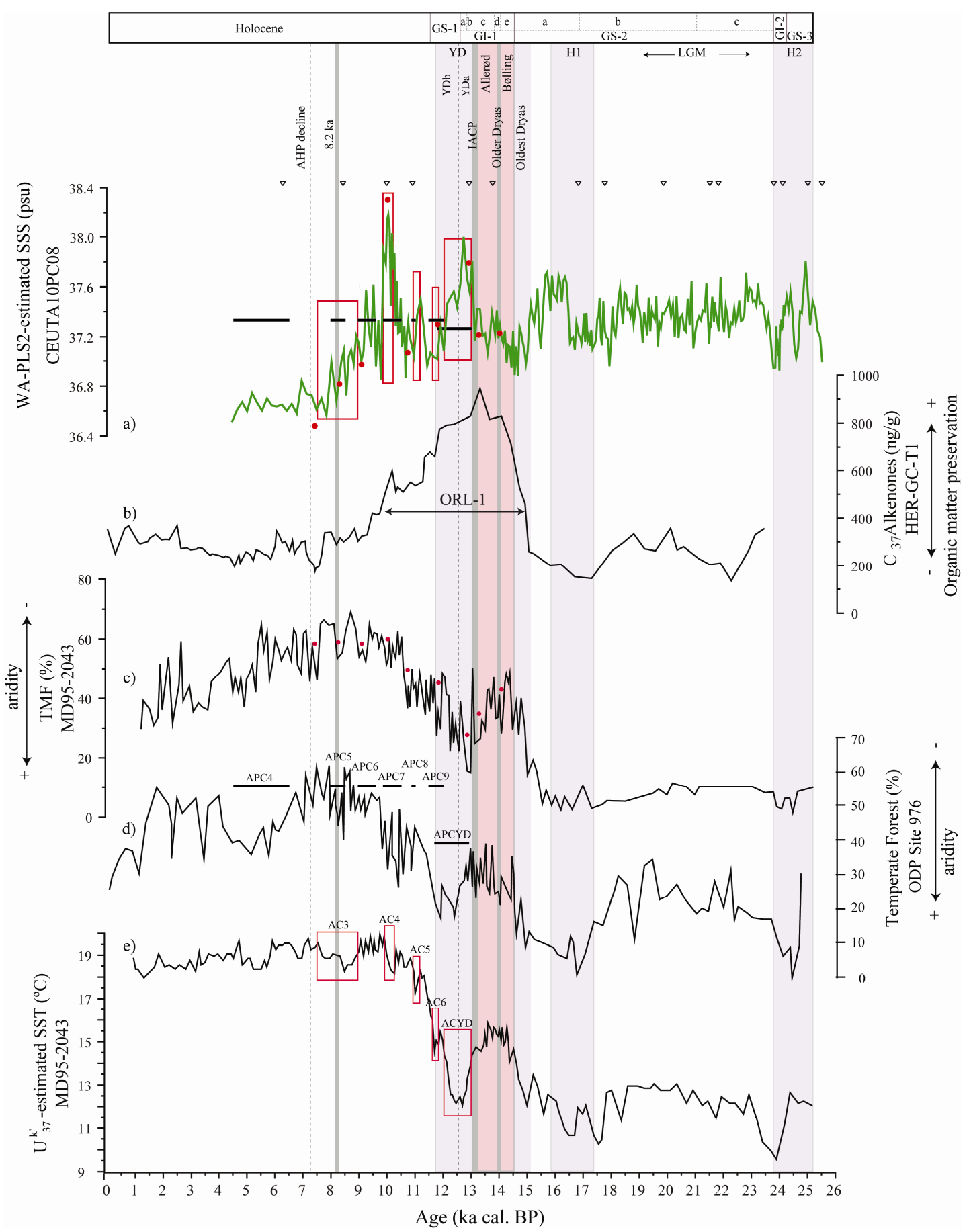

Figure 6.7. Paleoenvironmental records in the Alboran Sea for the last 25 kyr: a) SSS reconstruction (psu) for core CEUTA10PC08. b) $\mathrm{C}_{37}$ Alkenones (ng/g) from core HER-GC-T1 in the Alboran Sea (Ausín et al., 2015). c) Pollen percentages for temperate Mediterranean forest (TMF; \%) taxa from core MD95-2043 in the Alboran Sea (Fletcher et al., 2010). Red points indicate the central age of the periods of forest decline. d) Pollen record (\%) from ODP Site 976 in the Alboran Sea (Combourieu Nebout et al., 2009). Horizontal black lines cover the timing of periods of continental aridity (APC) from APCYD to APC4. e) SST $\left({ }^{\circ} \mathrm{C}\right)$ from core MD95-2043 (Cacho et al., 2001). Red boxes represent the timing of the brief Alboran cooling events (AC), from ACYD to AC3 (Table 6.3). H2 and H1: stadials associated with Heinrich events 2 and 1; LGM: Last Glacial Maximum; IACP: Intra-Alleröd Cold Period. YD: Younger Dryas. AHP: African Humid Period. 
In particular, Duplessy et al. (1992) (Fig. 6.6c) associated it with the Oldest Dryas in northwestern Europe and linked it to the meltwater release from the Barents and the Fennoscandinavian ice sheets. These authors also argued that such minor injections of freshwater would have been insufficient to trigger the observed drop in salinity, and proposed an additional feedback from changes in the hydrological cycle and water advection to promote changes in the thermohaline circulation and the observed changes in SSS.

\subsubsection{4.-Bølling-Allerød $(B-A)$}

The SSS values are generally low for the $\mathrm{B}-\mathrm{A}$, the Bølling being fresher than the Allerød, both separated by the Older-Dryas (GI-1d) interval (Fig. 6.7a). Owing to the global sea-level rise during the $\mathrm{B}-\mathrm{A}$, and specifically during the mwp-1A between 13.5 and $14.1 \mathrm{ka}$, a greater volume of AW would have entered through the Strait, decreasing the average SSS. In addition, wetter conditions would have contributed to this freshening. Enhanced rainfall and increased river discharge have already been inferred from diatom assemblages, sediment grain-size, pollen records, elemental ratios and coccolithophore records in the Western Mediterranean during the B-A (Bárcena et al., 2001; Frigola et al., 2008; Combourieu Nebout et al., 2009; RodrigoGámiz et al., 2011; Ausín et al., 2015; Martínez-Ruiz et al., 2015). This period of reduced salinity also coincides with the development of the Organic-Rich Layer (ORL-1) (Cacho et al., 2002) (Fig. 6.7b), which appears in many sediment cores from the Alboran Sea as a consequence of the accumulation of high levels of organic matter. Comparison of reconstructed SSS and the profile of the total concentration of $\mathrm{C}_{37}$ alkenones (a proxy of organic matter preservation) from a nearby core (Fig. 6.7a, b) allows the role of salinity in the formation of the ORL to be explored. Values below 37.25 psu match an increasing trend in organic matter preservation. The joint effect of a salinity reduction of $0.8 \mathrm{psu}$ and a temperature increase of 3 ${ }^{\circ} \mathrm{C}$ (Ausín et al., 2015) would have led to a significant reduction in sea surface density, possibly prompting stagnation of the upper water column. Moreover, Ausín et al. (2015) identified a rise in primary productivity in the Alboran Sea from fossil coccolithophore assemblages. Frigola et al. (2008) and Martínez-Ruiz et al. (2015) identified a reduction in deep water formation in the Gulf of Lion using sediment-grain size analyses and elemental ratios. Increased organic matter export to the seabed, along with reduced deep-basin ventilation, would have hampered organic matter mineralization, reinforcing the formation of the ORL. Rogerson et al. (2008) have used model simulations to explore the mechanisms driving ORL formation. These authors concluded that a strong reduction in seawater density, as demonstrated here, in conjunction with a rise of the interface between deep and intermediate waters, would be the most likely explanation for the origin of the Alboran ORL. 
The brief Intra-Alleröd Cold Period (IACP) (G1-1b) (Lehman and Keigmin, 1992) put an end to the low salinity values of the B-A. The SSS increasing trend is in agreement with the enhanced continental aridity reported for elemental ratios from a nearby core for this brief time span (Rodrigo-Gámiz et al., 2011) (Fig. 6.7a).

\subsubsection{6.-Younger Dryas (YD) and the Holocene}

The YD displays a shift from higher to lower SSS values, decreasing by $0.6 \mathrm{psu}$ in total along its two phases (YDa and $\mathrm{YDb}$ ). This is in keeping with a transition from arid to humid conditions (Cacho et al., 2001; Sbaffi et al., 2004; Combourieu Nebout et al., 2009; RodrigoGámiz et al., 2011). Several large short-term SSS fluctuations occurred as from the onset of the YD up to $8 \mathrm{ka}$ (Fig. 6.7a). This time span coincides exactly with the highest summer insolation values of the last $25 \mathrm{kyr}$ (threshold value above $\sim 7 \%$ of current values), with a period of continuous SST increasing trend (Cacho et al., 2001) (Fig. 6.7e) and the rise in the post-glacial sea level (Fairbanks, 1989). However, fast oscillations point to an intermittent influence of the forcing mechanisms. SSS changes in the Western Mediterranean can be driven by atmospheric and/or oceanic processes. With regard to atmospheric conditions, an alternation between moist and dry periods as a control for these SSS changes is not fully supported by pollen data from the Western Mediterranean (Combourieu Nebout et al., 2009; Dormoy et al., 2009; Fletcher et al., 2010). Some of these pollen profiles reveal the occurrence of periods of greater dryness in the Western Mediterranean, but they do not coincide exactly with SSS increases (Fig. 6.7a, c, d), as would be expected from reduced rainfall and decreased river discharge. Regarding oceanic processes, variations in the salinity and flux of the incoming AW are able to drive salinity changes in the study area. Owing to its proximity to the Strait, core CEUTA10PC08 is expected to be a sensitive record to salinity changes in the past driven by AW influxes. Thus, periods of less saline AW influx should be recognizable in the SSS reconstruction. Six brief periods of an SSS decreasing trend were identified at 13.07-12.06, 11.95-11.71, 11.36-11.00, 10.09-9.83, 9.57-9.21, and 8.95-7.72 ka (Fig. 6.7a). Except for 9.57-9.21 ka, all of them are accurately simultaneous to the brief Alboran cooling (AC) events (Table 6.3, Fig. 6.7e) that have been linked to North Atlantic influxes in the Alboran Sea (Cacho et al., 2001). The synchronicity of the SST and SSS drops suggests a common origin. Short North Atlantic cold and freshwater influxes are the most likely explanation. AC events have been correlated with those of declining forest (named APC) deduced from pollen analyses by Combourieu Nebout et al. (2009) (Table 6.3, Fig. 6.7a, d,) and Fletcher et al. (2010) (Fig. 6.7a, c), suggesting increased aridity inland at times of freshwater advection events (FA; Table 6.3). The APC6 dry period from 9.6 to $8.9 \mathrm{ka}$ (Table 6.3, Fig. 6.7d) (Combourieu Nebout et al., 2009) does not match any AC event, but coincides with FA2 (Table 6.3), demonstrating the common nature of these short-term oscillations. 
The last freshening episode (FA1; 8.95-7.72 ka) includes the 8.2 ka event (Alley et al., 1997), which has been linked to a sub-thermocline freshening of $0.5 \mathrm{psu}$ in the North Atlantic (Thornalley et al., 2009). However, no conspicuous changes are observed in reconstructed SSS at that precise time. Zanchetta et al. (2007) inferred episodes of increased rainfall from highresolution oxygen isotopic records in a stalagmite from the Corchia cave (Central Italy). These authors found no evidence of the $8.2 \mathrm{ka}$ event in relation to SSS. Similarly, we interpret that the $8.2 \mathrm{ka}$ event would have had a negligible effect on surface salinity in the Alboran Sea. The aforementioned FA1 ends at $7.4 \mathrm{ka}$, when the lowest values SSS are recorded. At this time, the sea level in the Alboran Sea reached maximum high-stand conditions (Zazo et al., 2008). Moreover, the African Humid Period (11-5.5 ka) affected the study area, especially up to $7.4 \mathrm{ka}$, when it started to decline (Ritchie et al., 1985; deMenocal et al., 2000; Renssen et al., 2003). The joint action of these processes certainly resulted in a decrease of SSS at 7.4 ka. From 7.4 to $4.5 \mathrm{ka}$, salinity values level off around $36.6 \mathrm{psu}$, close to present SSS values in the Alboran Sea.

\section{Table 6.3}

Timing (given in ka cal. BP) of: freshwater advection events (FA) deduced from SSS decreases in core CEUTA10PC08 (this study); cooling events from core MD 95-2043 (ACYD-AC3, Cacho et al. (2001)), and continental aridity periods from core ODP Site 976 (YD-APC5, Combourieu Nebout et al., (2009)). All cores from the Alboran Sea.

\begin{tabular}{c|cc}
\hline SSS decreases & Cooling events & $\begin{array}{c}\text { Continental aridity } \\
\text { periods }\end{array}$ \\
\hline \hline FA6 & ACYD & YD \\
$\mathbf{1 3 . 1 - 1 2 . 0}$ & $13.1-12.0$ & $12.8-11.75$ \\
FA5 & AC6 & APC9 \\
$\mathbf{1 1 . 9 - 1 1 . 7}$ & $11.9-11.65$ & $11.75-11.4$ \\
FA4 & AC5 & APC8 \\
$\mathbf{1 1 . 4 - 1 1 . 0}$ & $11.21-10.95$ & $11.0-10.8$ \\
FA3 & AC4 & APC7 \\
$\mathbf{1 0 . 1 - 9 . 8}$ & $10.34-9.95$ & $10.5-9.8$ \\
FA2 & --- & APC6 \\
$\mathbf{9 . 6 - 9 . 2}$ & & $9.6-8.9$ \\
FA1 & AC3 & APC5 \\
$\mathbf{9 . 0 - 7 . 7}$ & $9.08-7.56$ & $8.5-7.9$
\end{tabular}




\section{6.-CONCLUSIONS}

Statistical analyses show that coccolithophore distribution in this training set was mainly driven by annual mean salinity at $10 \mathrm{~m}$ depth. MAT and WA-PLS2 calibration models afford similar outcomes. These models were applied to coccolithophore assemblages from a fossil core to reconstruct SSS at high resolution for the last $25 \mathrm{kyr}$ in the Alboran Sea. Reconstructed SSS show similar patterns to other records from nearby locations and their validity and reliability can be demonstrated statistically by evaluating the degree of similarity between fossil and modern coccolithophore assemblages. Statistical analyses reveal assemblages lacking analogs in relation to the species E. huxleyi $>4 \mu \mathrm{m}$ during $\mathrm{H} 2$ and $\mathrm{H} 1$ and part of the LGM, preventing further interpretations for these periods. SSS was relatively high from 23.5 to $21 \mathrm{ka}$, likely due to a limited Atlantic-Mediterranean connection. A low SSS was found for the B-A, possibly due to the post-glacial sea-level rise and regionally wetter conditions in the adjacent continental areas. The consequent reduction in sea surface density is suggested to have triggered the formation of the ORL in the Alboran Sea. During the YD and Holocene, six brief, abrupt SSS decreases at 13.1-12.0, 11.9-11.7, 11.4-11.0, 10.1-9.8, 9.6-9.2, and 9.0-7.7 ka were linked to the advection of fresher AW related to meltwater pulses in the North Atlantic and continental aridity. No evidence of the $8.2 \mathrm{ka}$ event is found in the reconstructed SSS, which reached its lowest values at $7.4 \mathrm{ka}$, coinciding with high-stand conditions in the Alboran Sea and the onset of the decline of the African Humid Period. SSS remained low from 7.4 to $4.5 \mathrm{ka}$, close to its present values.

A broader understanding of the ecological link between coccolithophore species and environmental parameters would be desirable in order to be able to place coccolithophore-based transfer functions within the ecological context in future works. Nevertheless, the diverse statistical tests performed in this study and the strong emphasis placed on assessing the validity and reliability of both the model and the reconstruction do reveal the potential of coccolithophores for developing transfer functions. The information about the salinity preferences of coccolithophore species described here provides a potential independent proxy for salinity reconstructions. The derived transfer function could be a useful tool for quantitative reconstructions of SSS changes over time in other locations of the Western Mediterranean Sea, especially from $15 \mathrm{ka}$ onwards.

\section{Acknowledgements}

B. Ausín is sincerely grateful to the Core Repository of the Institute of Marines SciencesCSIC at Barcelona and the University of Vigo for the core-top samples supply. This study was supported by the FPU grant AP2010-2559 of the Ministry of Education of Spain given to B. Ausín and by the Consolider Ingenio "GRACCIE" program CSD 2007-00067, the program SA263U14 of Junta de Castilla y León, and the programs: CGL2011-26493, VACLIODP339, 
CTM2008-06399-C04/MAR, CTM2012-39599-03-01 and MOWER (CTM 2012-39599-CO302/03) of the Spanish Ministry of Science and Innovation.

\section{References}

Alley RB, Mayewski PA, Sowers T, Stuiver M, Taylor KC and Clark PU. (1997) Holocene climatic instability: A prominent, widespread event 8200 yr ago. Geology 25: 483-486.

Ausín B, Flores JA, Bárcena MA, Sierro FJ, Francés G, Gutiérrez-Arnillas E, HernándezAlmeida I, Martrat B, Grimalt JO and Cacho I. (2015) Coccolithophore productivity and surface water dynamics in the Alboran Sea during the last $25 \mathrm{kyr}$. Palaeogeography, Palaeoclimatology, Palaeoecology 418: 126-140.

Bárcena MA, Cacho I, Abrantes F, Sierro FJ, Grimalt JO and Flores JA. (2001) Paleoproductivity variations related to climatic conditions in the Alboran Sea (western Mediterranean) during the last glacial-interglacial transition: the diatom record. Palaeogeography, Palaeoclimatology, Palaeoecology 167: 337-357.

Bard E, Hamelin B, Arnold M, Montaggioni L, Cabioch G, Faure G and Rougerie F. (1996) Deglacial sea-level record from Tahiti corals and the timing of global meltwater discharge. Nature 382: 241-244.

Baumann KH, Andruleit H, Boeckel B, Geisen M and Kinkel H. (2005) The significance of extant coccolithophores as indicators of ocean water masses, surface water temperature, and palaeoproductivity: a review. Palaeontologische Zeitschrift 79: 93-112.

Beaufort L, de Garidel-Thoron T, Mix AC and Pisias NG. (2001) ENSO-like Forcing on Oceanic Primary Production During the Late Pleistocene. Science 293: 2440-2444.

Beaufort L, Lancelot Y, Camberlin P, Cayre O, Vincent E, Bassinot F and Labeyrie L. (1997) Insolation Cycles as a Major Control of Equatorial Indian Ocean Primary Production. Science 278: 1451-1454.

Bèthoux JP. (1979) Budgets of the Mediterranean Sea. Their dependance on the local climate and on the characteristics of the Atlantic waters. Oceanologica Acta 2: 157-163.

Birks HJB. (1995) Quantitative palaeoenvironmental reconstructions. In: Maddy DB, J.S. (ed) Statistical Modelling of Quaternary Science Data. Technical guide 5, Quaternary Research Association. Cambridge, 271.

Blanz T, Emeis KC and Siegel H. (2005) Controls on alkenone unsaturation ratios along the salinity gradient between the open ocean and the Baltic Sea. Geochimica et Cosmochimica Acta 69: 3589-3600.

Bollmann J and Herrle JO. (2007) Morphological variation of Emiliania huxleyi and sea surface salinity. Earth and Planetary Science Letters 255: 273-288. 
Bollmann J, Herrle JO, Cortés MY and Fielding SR. (2009) The effect of sea water salinity on the morphology of Emiliania huxleyi in plankton and sediment samples. Earth and Planetary Science Letters 284: 320-328.

Boyer TP, Antonov JI, Baranova OK, Coleman C, García HE, Grodsky A, Johnson DR, Locarnini RA, Mishonov AV, O'Brien TD, Paver CR, Reagan JR, Seidov D, Smolyar IV and Zweng MM. (2013) World Ocean Database 2013, NOAA Atlas NESDIS 72.

Cacho I, Grimalt JO and Canals M. (2002) Response of the Western Mediterranean Sea to rapid climatic variability during the last 50,000 years: a molecular biomarker approach. Journal of Marine Systems 33-34: 253-272.

Cacho I, Grimalt JO, Canals M, Sbaffi L, Shackleton NJ, Schönfeld J and Zahn R. (2001) Variability of the western Mediterranean Sea surface temperature during the last 25,000 years and its connection with the Northern Hemisphere climatic changes. Paleoceanography 16: 40-52.

Cacho I, Grimalt JO, Pelejero C, Canals M, Sierro FJ, Flores JA and Shackleton N. (1999) Dansgaard-Oeschger and Heinrich event imprints in Alboran Sea paleotemperatures. Paleoceanography 14: 698-705.

CLIMAP Project Members. (1976) The Surface of the Ice-Age Earth. Science 191: 1131-1137.

Colmenero-Hidalgo E, Flores JA, Sierro FJ, Bárcena MÁ, Löwemark L, Schönfeld J and Grimalt JO. (2004) Ocean surface water response to short-term climate changes revealed by coccolithophores from the Gulf of Cadiz (NE Atlantic) and Alboran Sea (W Mediterranean). Palaeogeography, Palaeoclimatology, Palaeoecology 205: 317-336.

Combourieu Nebout N, Peyron O, Dormoy I, Desprat S, Beaudouin C, Kotthoff U and Marret F. (2009) Rapid climatic variability in the west Mediterranean during the last 25000 years from high resolution pollen data. Climate of the Past 5: 503-521.

Cros L and Fortuño JM. (2002) Atlas of northwestern Mediterranean coccolithophores. Scientia Marina 66: 7-182.

Dafner EV, Boscolo R and Bryden HL. (2003) The N:Si:P molar ratio in the Strait of Gibraltar. Geophysical Research Letters 30: 1506.

deMenocal P, Ortiz J, Guilderson T, Adkins J, Sarnthein M, Baker L and Yarusinsky M. (2000) Abrupt onset and termination of the African Humid Period: rapid climate responses to gradual insolation forcing. Quaternary Science Reviews 19: 347-361.

Dormoy I, Peyron O, Combourieu Nebout N, Goring S, Kotthoff U, Magny M and Pross J. (2009) Terrestrial climate variability and seasonality changes in the Mediterranean region between 15,000 and 4,000 years BP deduced from marine pollen records. Climate of the Past 5: 615-632. 
Duplessy JC, Labeyrie L, Arnold M, Paterne M, Duprat J and van Weering TCE. (1992) Changes in surface salinity of the North Atlantic Ocean during the last deglaciation. Nature 358: 485-488.

Edwards RJ, van de Plassche O, Gehrels WR and Wright AJ. (2004) Assessing sea-level data from Connecticut, USA, using a foraminiferal transfer function for tide level. Marine Micropaleontology 51: 239-255.

Emeis KC, Struck U, Schulz HM, Rosenberg R, Bernasconi S, Erlenkeuser H, Sakamoto T and Martínez-Ruiz F. (2000) Temperature and salinity variations of Mediterranean Sea surface waters over the last 16,000 years from records of planktonic stable oxygen isotopes and alkenone unsaturation ratios. Palaeogeography, Palaeoclimatology, Palaeoecology 158: 259-280.

Essallami L, Sicre MA, Kallel N, Labeyrie L and Siani G. (2007) Hydrological changes in the Mediterranean Sea over the last 30,000 years. Geochemistry, Geophysics, Geosystems 8: Q07002.

Fairbanks RG. (1989) A 17,000-year glacio-eustatic sea level record; influence of glacial melting rates on the Younger Dryas event and deep-ocean circulation. Nature 342: 637642.

Fielding SR, Herrle JO, Bollmann J, Worden RH and Montagnesd DJS. (2009) Assessing the applicability of Emiliania huxleyi coccolith morphology as a sea-surface salinity proxy. Limnology and Oceanography 54: 1475-1480.

Fletcher WJ, Sánchez Goñi MF, Peyron O and Dormoy I. (2010) Abrupt climate changes of the last deglaciation detected in a Western Mediterranean forest record. Climate of the Past 6: 245-264.

Fletcher WJ and Sánchez Goñi MF. (2008) Orbital- and sub-orbital-scale climate impacts on vegetation of the western Mediterranean basin over the last 48,000 yr. Quaternary Research 70: 451-464.

Flores J, Sierro F, Francés G, Vázquez A and Zamarreño I. (1997) The last 100,000 years in the western Mediterranean: sea surface water and frontal dynamics as revealed by coccolithophores. Marine Micropaleontology 29: 351-366.

Flores JA and Sierro FJ. (1997) Revised technique for calculation of calcareous nannofossil accumulation rates. Micropaleontology 43: 321-324.

Font J, García-Ladona E and Gorriz E. (1995) The seasonality of mesoscale motion in the Northern Current of the western Mediterranean: several years of evidence. Oceanologica Acta 18: 207-219.

Font J, Salat J and Tintore J. (1988) Permanent features of the circulation in the Catalan Sea. Oceanologica Acta 9: 51-57. 
Frigola J, Moreno A, Cacho I, Canals M, Sierro FJ, Flores JA and Grimalt JO. (2008) Evidence of abrupt changes in Western Mediterranean deep water circulation during the last 50 kyr: a high-resolution marine record from the Balearic Sea. Quaternary International 181: 88-104.

Fujine K, Yamamoto M, Tada R and Kido Y. (2006) A salinity-related occurrence of a novel alkenone and alkenoate in Late Pleistocene sediments from the Japan Sea. Organic Geochemistry 37: 1074-1084.

García HE, Locarnini RA, Boyer TP, Antonov JI, Baranova OK, Zweng MM, Reagan JR and Johnson DR. (2014a) World Ocean Atlas 2013, Volume 3: Dissolved Oxygen, Apparent Oxygen Utilization, and Oxygen Saturation. In: Levitus S and Mishonov A (eds) NOAA Atlas NESDIS 75, $27 \mathrm{pp}$.

García HE, Locarnini RA, Boyer TP, Antonov JI, Baranova OK, Zweng MM, Reagan JR and Johnson DR. (2014b) World Ocean Atlas 2013, Volume 4: Dissolved Inorganic Nutrients (phosphate, nitrate, silicate). In: Levitus S and Mishonov A (eds) NOAA Atlas NESDIS 76, 25 pp. 27.

Giraudeau J and Rogers J. (1994) Phytoplankton biomass and sea-surface temperature estimates from sea-bed distribution of nannofossils and planktonic foraminifera in the Benguela upwelling system. Micropaleontology 40: 275-285.

Green JC, Heimdal BR, Paasche E and Moate R. (1998) Changes in calcification and the dimensions of coccoliths of Emiliania huxleyi (Haptophyta) grown at reduced salinities. Phycologia 37: 121-131.

Guerreiro C, Oliveira A, de Stigter H, Cachão M, Sá C, Borges C, Cros L, Santos A, Fortuño JM and Rodrigues A. (2013) Late winter coccolithophore bloom off central Portugal in response to river discharge and upwelling. Continental Shelf Research 59: 65-83.

Hammer Ø, Harper DAT and Ryan PD. (2001) PAST: Paleontological statistics software package for education and data analysis. Palaeontologia Electronica. http://palaeoelectronica.org/2001_1/past/issue1_01.htm 4: 9.

Incarbona A, Di Stefano E, Patti B, Pelosi N, Bonomo S, Mazzola S, Sprovieri R, Tranchida G, Zgozi S and Bonanno A. (2008) Holocene millennial-scale productivity variations in the Sicily Channel (Mediterranean Sea). Paleoceanography 23: PA3204.

Jansson IM, Mertens KN, Head MJ, de Vernal A, Londeix L, Marret F, Matthiessen J and Sangiorgi F. (2014) Statistically assessing the correlation between salinity and morphology in cysts produced by the dinoflagellate Protoceratium reticulatum from surface sediments of the North Atlantic Ocean, Mediterranean-Marmara-Black Sea region, and Baltic-Kattegat-Skagerrak estuarine system. Palaeogeography, Palaeoclimatology, Palaeoecology 399: 202-213. 
Jiang H, Knudsen MF, Seidenkrantz MS, Zhao M, Sha L and Ran L. (2014) Diatom-based reconstruction of summer sea-surface salinity in the South China Sea over the last 15,000 years. Boreas 43: 208-219.

Juggins S. (2007) C2 Version 1.5 Software for ecological and palaeoecological data analysis and visualisation. In: University $\mathrm{N}$ (ed). Newcastle upon Tyne, UK.

Juggins S. (2013) Quantitative reconstructions in palaeolimnology: new paradigm or sick science? Quaternary Science Reviews 64: 20-32.

Kallel N, Paterne M, Labeyrie L, Duplessy J-C and Arnold M. (1997) Temperature and salinity records of the Tyrrhenian Sea during the last 18,000 years. Palaeogeography, Palaeoclimatology, Palaeoecology 135: 97-108.

Knappertsbusch M. (1993) Geographic distribution of living and Holocene coccolithophores in the Mediterranean Sea. Marine Micropaleontology 21: 219-247.

Lehman SJ and Keigmin LD. (1992) Sudden changes in Norht Atlantic circulation during the last glaciation. Nature 356: 757-762.

Li D, Knudsen MF, Jiang H, Olsen J, Zhao M, Li T, Knudsen KL, Seidenkrantz M-S and Sha L. (2012) A diatom-based reconstruction of summer sea-surface salinity in the Southern Okinawa Trough, East China Sea, over the last millennium. Journal of Quaternary Science 27: 771-779.

Locarnini R, Mishonov A, Antonov J, Boyer T, García H, Baranova O, Zweng M, Paver CR, Reagan JR, Johnson DR, Hamilton M and Seidov D. (2013) World Ocean Atlas 2013, Volume 1: Temperature. In: Levitus S and Mishonov A (eds) NOAA Atlas NESDIS 73, $40 \mathrm{pp}$.

Martínez-Ruiz F, Kastner M, Gallego-Torres D, Rodrigo-Gámiz M, Nieto-Moreno V and Ortega-Huertas M. (2015) Paleoclimate and paleoceanography over the past 20,000 yr in the Mediterranean Sea Basins as indicated by sediment elemental proxies. Quaternary Science Reviews 107: 25-46.

Martrat B, Grimalt JO, Lopez-Martínez C, Cacho I, Sierro FJ, Flores JA, Zahn R, Canals M, Curtis JH and Hodell DA. (2004) Abrupt Temperature Changes in the Western Mediterranean over the Past 250,000 Years. Science 306: 1762-1765.

Martrat B, Grimalt JO, Shackleton NJ, de Abreu L, Hutterli MA and Stocker TF. (2007) Four Climate Cycles of Recurring Deep and Surface Water Destabilizations on the Iberian Margin. Science 317: 502-507.

Melki T. (2011) Variation of deepwater convection in the western Mediterranean Sea (Gulf of Lion) during the last $28 \mathrm{ka}$. Quaternary International 241: 160-168.

Millot C. (1992) Are there major differences between the largest mediterranean seas? A preliminary investigation. Bulletin de l'Institut Océanographique, Monaco 11: 3-25. 
Millot C. (1999) Circulation in the Western Mediterranean Sea. Journal of Marine Systems 20: 423-442.

Oksanen J, Kindt R, Legendre P, O’Hara B, Stevens MHH, Oksanen MJ and Suggests MASS. (2007) The vegan package. Community ecology package.

Overpeck JT, Webb T and Prentice IC. (1985) Quantitative interpretation of fossil pollen spectra: Dissimilarity coeffi cients and the method of modern analogs. Quaternary Research 23: 87-108.

Paasche E, Brubak S, Skattebøl S, Young JR and Green JC. (1996) Growth and calcification in the coccolithophorid Emiliania huxleyi (Haptophyceae) at low salinities. Phycologia: 35: 394-403.

Pérez-Folgado M, Sierro FJ, Flores JA, Cacho I, Grimalt JO, Zahn R and Shackleton N. (2003) Western Mediterranean planktonic foraminifera events and millennial climatic variability during the last $70 \mathrm{kyr}$. Marine Micropaleontology 48: 49-70.

Prell WL. (1985) The stability of low-latitude sea-surface temperatures: An evaluation of the CLIMAP reconstruction with emphasis on the positive SST anomalies. Department of Energy. Washington, D. C., 60.

R Development Core Team. (2007) R: A Language and Environment for Statistical Computing. R Foundation for Statistical Computing. Vienna, Austria.

R Development Core Team. (2011) R: A language and environment for statistical computing. R Foundation for Statistical Computing.

Rao CR. (1995) A review of canonical coordinates and an alternative to correspondence analysis using Hellinger distance. Qüestiió 19: 23-63.

Renssen H, Brovkin V, Fichefet T and Goosse H. (2003) Holocene climate instability during the termination of the African Humid Period. Geophysical Research Letters 30: 1184.

Ritchie JC, Eyles CH and Haynes CV. (1985) Sediment and pollen evidence for an early to midHolocene humid period in the eastern Sahara. Nature 330: 645-647.

Rodrigo-Gámiz M, Martínez-Ruiz F, Jiménez-Espejo FJ, Gallego-Torres D, Nieto-Moreno V, Romero O and Ariztegui D. (2011) Impact of climate variability in the western Mediterranean during the last 20,000 years: oceanic and atmospheric responses. Quaternary Science Reviews 30: 2018-2034.

Rodrigo-Gámiz M, Martínez-Ruiz F, Rampen SW, Schouten S and Sinninghe Damsté JS. (2014) Sea surface temperature variations in the western Mediterranean Sea over the last 20 kyr: A dual-organic proxy $\left(\mathrm{U}^{\mathrm{K}^{\prime}}{ }_{37}\right.$ and LDI) approach. Paleoceanography 29: 8798. 
Rogerson M, Cacho I, Jiménez-Espejo F, Reguera MI, Sierro FJ, Martínez-Ruiz F, Frigola J and Canals M. (2008) A dynamic explanation for the origin of the western Mediterranean organic-rich layers. Geochemistry, Geophysics, Geosystems 9: Q07U01.

Rohling EJ. (1999) Environmental control on Mediterranean salinity and $\delta^{18} \mathrm{O}$. Paleoceanography 14: 706-715.

Rohling EJ. (2000) Paleosalinity: confidence limits and future applications. Marine Geology 163: $1-11$.

Rohling EJ and Bigg GR. (1998) Paleosalinity and $\delta^{18} \mathrm{O}$ : A critical assessment. Journal of Geophysical Research: Oceans 103: 1307-1318.

Saavedra-Pellitero M, Baumann KH, Hernández-Almeida I, Flores JA and Sierro FJ. (2013) Modern sea surface productivity and temperature estimations off Chile as detected by coccolith accumulation rates. Palaeogeography, Palaeoclimatology, Palaeoecology 392: 534-545.

Saavedra-Pellitero M, Flores JA, Lamy F, Sierro FJ and Cortina A. (2011) Coccolithophore estimates of paleotemperature and paleoproductivity changes in the southeast Pacific over the past 27 kyr. Paleoceanography 26: PA1201.

Sbaffi L, Wezel FC, Curzi G and Zoppi U. (2004) Millennial- to centennial-scale palaeoclimatic variations during Termination I and the Holocene in the central Mediterranean Sea. Global and Planetary Change 40: 201-217.

Schlitzer R. (2014) Ocean Data View, http://odv.awi.de.

Schmidt GA. (1998) Oxygen-18 variations in a global ocean model. Geophysical Research Letters 25: 1201-1204.

Schmidt GA. (1999) Error analysis of paleosalinity calculations. Paleoceanography 14: 422429.

Schouten S, Ossebaar J, Schreiber K, Kienhuis MVM, Langer G, Benthien A and Bijma J. (2006) The effect of temperature, salinity and growth rate on the stable hydrogen isotopic composition of long chain alkenones produced by Emiliania huxleyi and Gephyrocapsa oceanica. Biogeosciences 3: 113-119.

Sierro FJ, Hodell DA, Curtis JH, Flores JA, Reguera I, Colmenero-Hidalgo E, Bárcena MA, Grimalt JO, Cacho I, Frigola J and Canals M. (2005) Impact of iceberg melting on Mediterranean thermohaline circulation during Heinrich events. Paleoceanography 20: PA2019.

Simpson GL. (2007) Analogue methods in palaeoecology: Using the analogue package. Journal of Statistical Software 22.

Telford R. (2012) palaeoSig: significance tests of quantitative palaeoenvironmental reconstructions. R Package Version, 1.1-1. 
Telford RJ, Andersson C, Birks HJB and Juggins S. (2004) Biases in the estimation of transfer function prediction errors. Paleoceanography 19: PA4014.

Telford RJ and Birks HJB. (2009) Evaluation of transfer functions in spatially structured environments. Quaternary Science Reviews 28: 1309-1316.

Telford RJ and Birks HJB. (2011) A novel method for assessing the statistical significance of quantitative reconstructions inferred from biotic assemblages. Quaternary Science Reviews 30: 1272-1278.

Telford RJ, Li C and Kucera M. (2013) Mismatch between the depth habitat of planktonic foraminifera and the calibration depth of SST transfer functions may bias reconstructions. Climate of the Past 9: 859-870.

ter Braak CJF and Juggins S. (1993) Weighted averaging partial least squares regression (WAPLS): an improved method for reconstructing environmental variables from species assemblages. Hydrobiologia 269-270: 485-502.

ter Braak CJF, Juggins S, Birks HJB and van der Voet. H. (1993) Weighted averaging partial least squares regresion (WA-PLS): definition and comparison with other methods for species-environment calibration. Chapter 25. In: Patil GP and Rao CR (eds) Multivariate Environmental Statistics. North-Holland, Amsterdam.

ter Braak CJF and Prentice IC. (1988) A Theory of Gradient Analysis. Advances in Ecological Research 18: 271-317.

Thornalley DJR, Elderfield H and McCave IN. (2009) Holocene oscillations in temperature and salinity of the surface subpolar North Atlantic. Nature 457: 711-714.

Weaver AJ, Saenko OA, Clark PU and Mitrovica JX. (2003) Meltwater Pulse 1A from Antarctica as a Trigger of the Bølling-Allerød Warm Interval. Science 299: 1709-1713.

Zanchetta G, Drysdale RN, Hellstrom JC, Fallick AE, Isola I, Gagan MK and Pareschi MT. (2007) Enhanced rainfall in the Western Mediterranean during deposition of sapropel S1: stalagmite evidence from Corchia cave (Central Italy). Quaternary Science Reviews 26: 279-286.

Zazo C, Dabrio CJ, Goy Goy JL, Lario J, Cabero del Río A, Silva Barroso PG, Bardají Azcárate T, Mercier N, Borja F and Roquero E. (2008) The coastal archives of the last $15 \mathrm{ka}$ in the Atlantic-Mediterranean Spanish linkage area: Sea level and climate changes. Quaternary International 181: 72-87.

Zweng MM, Reagan JR, Antonov JI, Locarnini RA, Mishonov AV, Boyer TP, García HE, Baranova OK, Johnson DR, Seidov D and Biddle MM. (2013) World Ocean Atlas 2013, Volume 2: Salinity. In: Levitus S and Mishonov A (eds) NOAA Atlas NESDIS 74, $39 \mathrm{pp}$. 


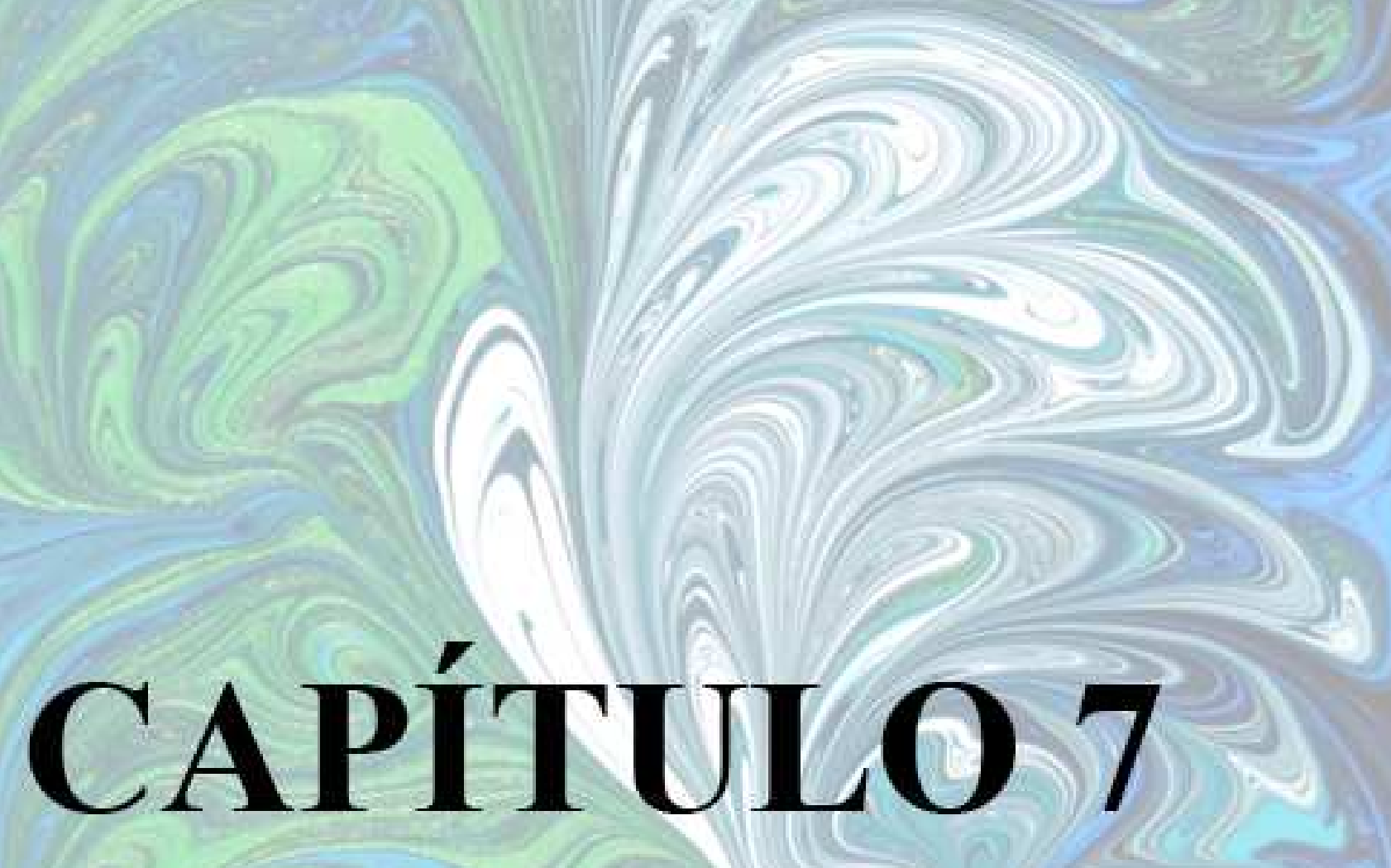





\section{7.-CONCLUSIONES}

El estudio a alta resolución de las asociaciones cocolitoforales presentes en dos testigos sedimentarios del mar de Alborán ha permitido obtener una valiosa información sobre los cambios en su productividad a lo largo de los últimos $25 \mathrm{ka}$ y evaluar la variabilidad de los factores que la controlan, como la disponibilidad de nutrientes y las condiciones e intensidad del proceso de afloramiento.

- El estudio de estos registros junto al de otros indicadores paleoambientales ha puesto de manifiesto la sensibilidad de los cocolitóforos a las variaciones hidrográficas y ambientales, tanto locales como regionales, permitiendo así deducir cambios en las dinámicas oceánicas y atmosféricas del Mediterráneo occidental durante los últimos $25 \mathrm{ka}$.

\section{1.-Variabilidad de la productividad de cocolitóforos y de la dinámica de la columna de agua durante los últimos 25 ka en el mar de Alborán}

- Los registros de abundancia absoluta de cocolitóforos, en combinación con registros de isótopos de oxígeno y SST a partir de foraminíferos planctónicos y alquenonas, han permitido reconstruir las propiedades del AW que entra a través del estrecho de Gibraltar. De este modo, se ha demostrado que esta masa de agua ejerce un control de primer orden sobre las variaciones de la productividad de cocolitóforos en las zonas más próximas al estrecho de Gibraltar. En localizaciones más distantes, la influencia de las propiedades del AW se disipa, ejerciendo un mayor control sobre la productividad de los cocolitóforos procesos más locales como la entrada de nutrientes por descarga fluvial, el afloramiento de agua profunda inducido por el viento o la hidrografía local.

- Estos registros, propios de la parte superior de la columna de agua, junto a los de algunos marcadores orgánicos (concentración de alquenonas e índice alcohol) relativos a las condiciones de la parte inferior de la columna de agua, han permitido describir los cambios en la productividad de cocolitóforos y en las dinámicas de la columna de agua para los últimos $25 \mathrm{ka}$ :

-Durante el H2 y el H1, la entrada de agua más fría y menos salina habría promovido la estratificación de la parte superior de la columna de agua impidiendo la productividad de cocolitóforos.

-Entre 21 y $18 \mathrm{ka}$, se propone el incremento de la descarga fluvial en el mar de Alborán como el mecanismo que explica la presencia conjunta de aguas más frías y menos salinas y un aumento en la productividad de cocolitóforos.

-Las fases T1a y T1b de la deglaciación se habrían caracterizado por una tendencia creciente de la SST y una columna de agua estable, aunque este hecho no habría impedido eventos productivos, posiblemente producidos por afloramientos costeros. 
-El Bølling-Allerød se caracterizó por un pequeño incremento de la productividad y por el desarrollo de la ORL.

-Se observan dos fases del Younger Dryas: una primera (YDa) más fría y una segunda (YDb) más cálida y húmeda. Durante ambas fases se observan diferencias en la productividad de cocolitóforos entre ambas localizaciones, posiblemente debidas a la influencia local de las propiedades del AW y las descargas fluviales.

-El Holoceno, lejos de ser un periodo estable en términos de productividad, presenta grandes diferencias entre una y otra localización, posiblemente debidas a la configuración hidrográfica que persiste actualmente en el mar de Alborán.

\section{2.-Variabilidad de la productividad de cocolitóforos durante el Holoceno en el mar de} Alborán

- El estudio a alta resolución de los registros de las tasas de acumulación de cocolitóforos (NAR) en la "célula productiva de Málaga" muestra que su productividad está estrechamente ligada a otros procesos de naturaleza física. El establecimiento de esta célula se propone a los 7,7 ka a partir de un aumento brusco en el NAR de la mayoría de las especies y en la ventilación del fondo de la cuenca.

- A partir de 7,7 ka en adelante, el registro de SST muestra una tendencia de enfriamiento. También se observa una disminución en la productividad de cocolitóforos en términos generales, aunque afectada por una mayor variabilidad, alternando periodos de debilitamiento e intensificación de las condiciones de afloramiento que provocan la eclosión de estos organismos. Estas variaciones muestran gran sincronía con los periodos de intensificación de la formación de la WMDW en el golfo de León y por tanto con los patrones de variabilidad atmosférica.

- Se han propuesto dos fases alternantes de un mismo escenario para describir las principales características de la variabilidad climática y oceanográfica y su relación con la de la productividad de cocolitóforos prevalecientes en el mar Mediterráneo occidental durante los últimos 7,7 ka a escala secular:

i) Junto a condiciones climáticas más áridas, un debilitamiento de los vientos Mistral y Tramontana en el golfo de León habría resultado en una reducción de la formación de WMDW, que habría coincidido con un menor volumen o intensidad del AJ. Estas condiciones favorecerían la prevalencia de una columna de agua más estable dificultando eventos productivos.

ii) Junto a condiciones climáticas más húmedas, un fortalecimiento de los vientos Mistral y Tramontana habría provocado una intensificación en la producción de 
WMDW, sincrónico con un mayor volumen o intensidad del AJ. En consecuencia, el AJ migraría hacia el sur promoviendo en el norte el afloramiento de aguas más profundas que fertilizarían la zona y favorecerían los eventos productivos.

- El patrón invernal de la NAO es propuesto como un mecanismo altamente influyente sobre los procesos explicados por ambas fases del escenario. Estos resultados resaltan la sensibilidad del mar Mediterráneo occidental a los patrones climáticos de las latitudes altas.

7.3.-Desarrollo de funciones de transferencia para el cálculo de la salinidad superficial marina a partir de cocolitóforos

- Los cocolitóforos han probado ser organismos con un gran potencial para el desarrollo de funciones de transferencia.

- La distribución de los cocolitóforos en la base de datos actual del mar Mediterráneo occidental probó estar influenciada principalmente por la salinidad, permitiendo desarrollar y aplicar una función de transferencia a un testigo sedimentario del mar de Alborán y reconstruir esta variable para los últimos $25 \mathrm{ka}$. Tanto la adecuación del modelo de regresión y calibración como la fiabilidad de la reconstrucción fueron evaluados estadísticamente mostrando buenos resultados.

- La reconstrucción para la SSS muestra varios cambios a escala secular y milenaria:

-Durante el H2 y el H1 se observa una disminución de la SSS. Sin embargo, estos periodos deben interpretarse con precaución debido a la falta de buenos análogos para la especie E. huxleyi $(>4 \mu \mathrm{m})$.

-Entre 23,5 y 21 ka la SSS era relativamente elevada. La disminución de 120 metros del nivel del mar respecto a su valor actual posiblemente provocó una reducción del intercambio de aguas entre el Atlántico y el Mediterráneo, favoreciendo un aumento de la salinidad en la cuenca mediterránea.

-La reducción de SSS observada durante el Bølling-Allerød parece haberse debido a la subida del nivel del mar y a condiciones atmosféricas más húmedas en la zona continental adyacente. Esta reducción de la SSS, junto a un aumento de la SST de $3{ }^{\circ} \mathrm{C}$, habría provocado un marcado descenso de la densidad en la parte superior de la columna de agua y la estratificación severa de ésta. Este proceso se propone como el principal desencadenante de la formación de la ORL en el mar de Alborán.

-A partir del Younger Dryas y durante el Holoceno se han identificado seis descensos bruscos de SSS durante 13,1-12,0;11,9-11,7; 11,4-11,0;10,1-9,8; 9,6-9,2; y 9,0-7,7 ka, sincrónicos con los periodos de enfriamiento del mar de Alborán y periodos de aridez continental. Esta sincronía sugiere un origen común, y se ha propuesto como explicación más 
plausible pulsos de advección de AW procedente del deshielo en el Atlántico norte. Estos resultados ponen de manifiesto una vez más la estrecha interrelación climática y oceanográfica entre la cuenca mediterránea y el Atlántico norte.

-Los valores de SSS más bajos se alcanzan en 7,4 ka, momento en el cual ha sido registrado el estadio de mayor nivel del mar en la cuenca de Alborán. A partir de 7,4 ka hasta la última parte del registro $(4,5 \mathrm{ka})$ los valores de SSS permanecen bajos y próximos a los valores actuales.

- El modelo de calibración de salinidad obtenido es la primera función de transferencia basada en cocolitóforos desarrollada en el mar Mediterráneo occidental, y se configura como una nueva herramienta para la obtención de registros de paleosalinidad a alta resolución en esta región, especialmente para los últimos $15 \mathrm{ka}$. A este respecto, se abre una línea de investigación futura que persiga los siguientes objetivos: 1) refinar el ajuste y capacidad predictiva de la función de transferencia mediante la ampliación de la base de datos actual y 2) aplicar la función a las asociaciones cocolitoforales fósiles de otros testigos sedimentarios del área de estudio para obtener nuevos registros de paleosalinidad pertenecientes al Mediterráneo occidental y estudiar su variabilidad. 
ANEXOS 



\section{ANEXO I.-LÁMINA FOTOGRÁFICA}

Imágenes de los cocolitos pertenecientes a los taxones más relevantes contemplados en este estudio y descritos en el capítulo 2
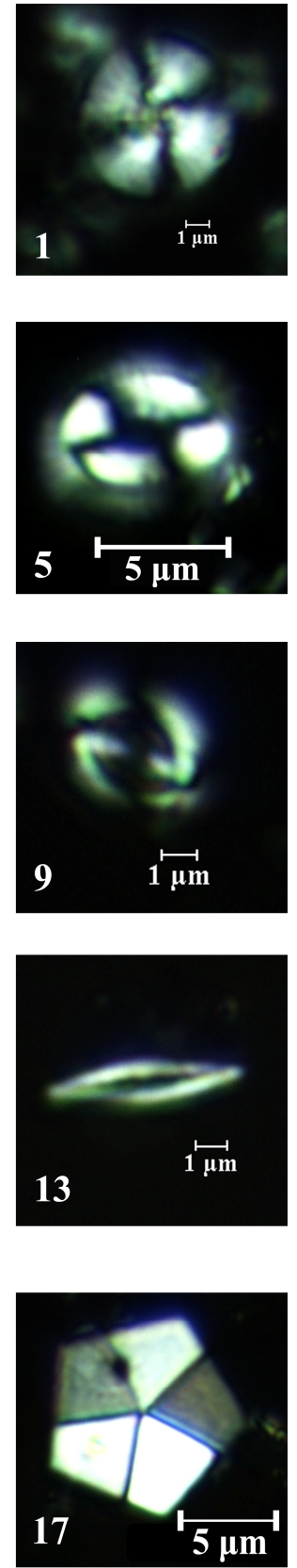
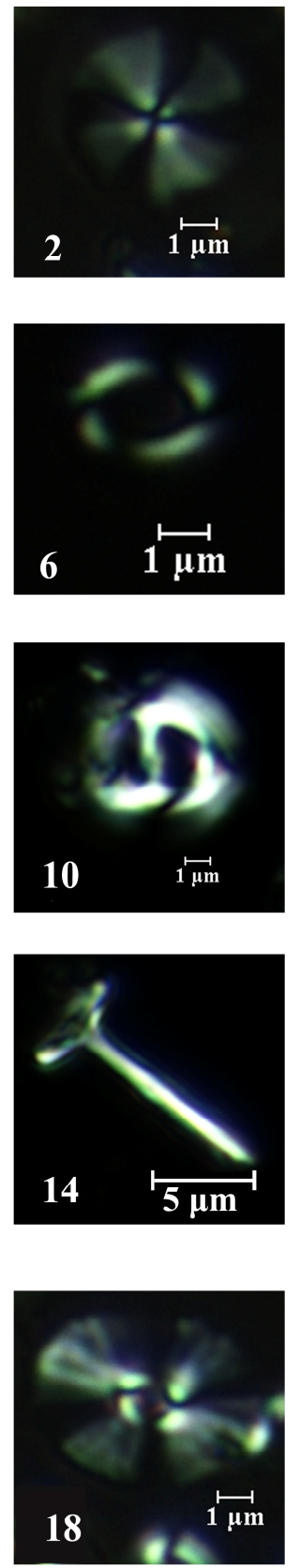
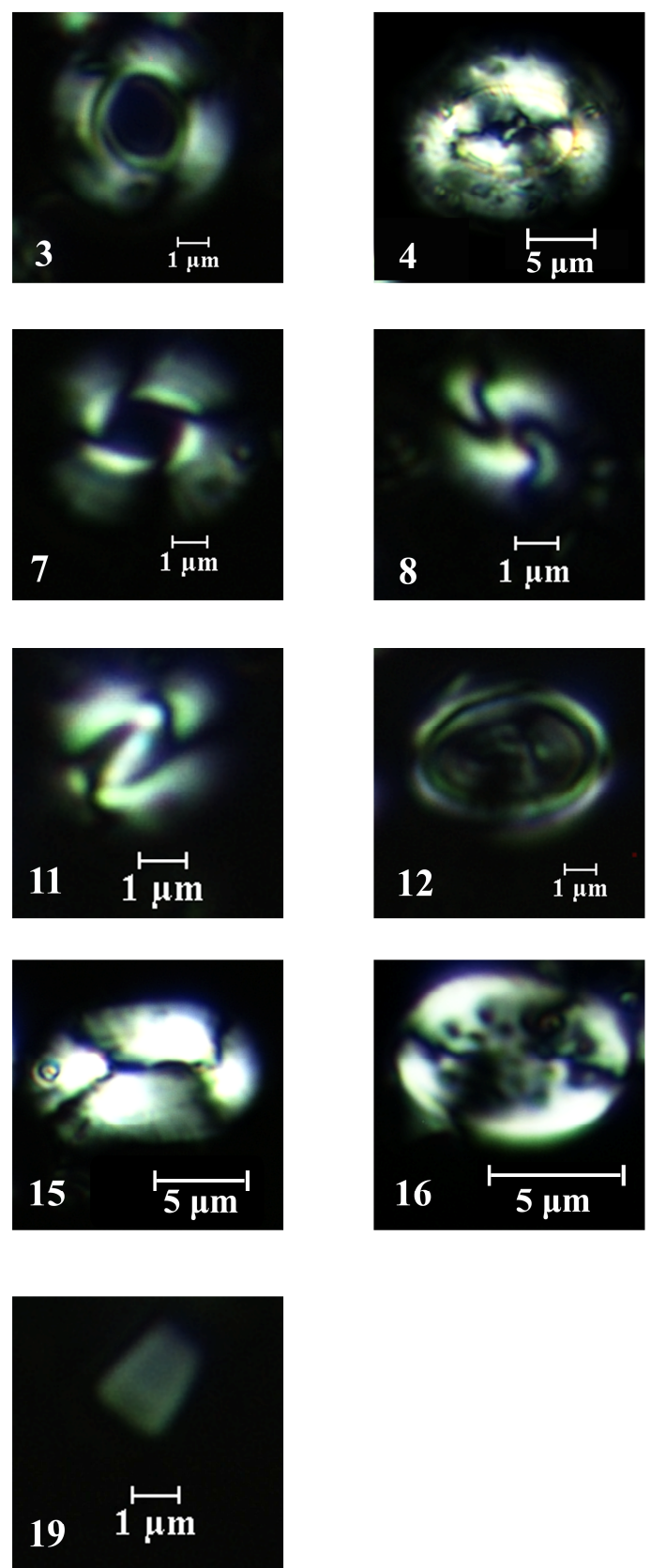

1: Calcidiscus leptoporus; 2: Oolithotus fragilis; 3: Umbilicosphaera sibogae; 4: Coccolithus pelagicus subespecie pelagicus; 5: Coccolithus pelagicus subespecie braarudii; 6: Emiliania huxleyi; 7: Emiliania huxleyi > $4 \mu \mathrm{m}$, 8: Gephyrocapsa caribbeanica; 9: Gephyrocapsa muellerae; 10: Gephyrocapsa oceanica; 11: "small" Gephyrocapsa; 12: Syracosphaera pulchra; 13: Calciosolenia murrayi; 14: Rhabdosphaera clavigera; 15: Helicosphaera carteri; 16: Pontosphaera multipora; 17: Braarudosphaera bigelowii; 18: Umbellosphaera irregularis; 19: Florisphaera profunda. 


\section{ANEXO II.-PROCEDIMIENTOS ADOPTADOS PARA LOS ANÁLISIS MICROPALEONTOLÓGICOS}

\section{Consideraciones previas:}

- Liofilización de las muestras: las 143 muestras de sedimento pertenecientes al testigo HER-GC-T1, las 297 muestras del testigo CEUTA10PC08 y las 118 que conforman la base de datos biológica y que se encontraban congeladas fueron liofilizadas por tandas de 35-40 muestras durante 17 horas aproximadamente para retirar todo el agua del sedimento húmedo.

- Con el fin de evitar la posible contaminación entre muestras será necesario enjaguar la espátula después de cada muestra en una disolución acuosa con unas gotas de ácido clorhídrico $(\mathrm{HCl})$ al $37 \%$, que disuelve cualquier resto de calcita adherido a la espátula. Después se enjuaga de nuevo la espátula con agua destilada y se seca con papel.

- Preparación del agua tamponada: disolver $0,15 \mathrm{~g}_{\text {de }} \mathrm{Na}_{2} \mathrm{CO}_{3}$ y $0,20 \mathrm{~g}$ de $\mathrm{NaHCO}_{3}$ en $1 \mathrm{~L}$ de agua destilada.

- Preparación de la gelatina: añadir 0,08 g de gelatina en $1 \mathrm{~L}$ de agua tamponada. Para facilitar la correcta disolución de la gelatina es conveniente que parte del agua tamponada se haya templado previamente. Es importante que no se caliente demasiado o las proteínas de la gelatina podrían desnaturalizarse. Una vez preparada, guardar en la nevera durante al menos 8 horas.

Técnica de decantación (Flores y Sierro 1997):

1. Para cada una de las muestras, pesar $0,1 \mathrm{~g}$ de sedimento seco en la balanza de precisión con la ayuda de una espátula metálica y un vidrio de reloj.

2. Introducir los 0,1 g de sedimento en un vial de cristal previamente etiquetado con el nombre de la muestra.

3. Añadir en cada vial $10 \mathrm{~mL}$ de agua tamponada.

4. Transcurridas más de 12 horas, agitar cada uno de los viales de vidrio y someterlos a ultrasonidos durante 15-20 segundos para disgregar la muestra.

5. Numerar una placa de Petri por cada muestra, colocar un cubre en su fondo y rellenarla con gelatina. La gelatina evita que la elevada tensión superficial del agua acumule partículas en el borde de la placa.

6. Dejar reposar cada bote unos segundos para seguidamente extraer $100 \mu \mathrm{L}$ de la parte media con ayuda de una micropipeta, evitando extraer así partículas de mayor tamaño que los cocolitos. 
7. Inyectar el volumen pipeteado en la gelatina de la placa de Petri, procurando que la disolución resultante sea homogénea con ayuda de la micropipeta para mezclar ambas disoluciones sin tocar ni la placa ni el cubre.

8. Dejar reposar las placas en una superficie horizontal durante 12 horas aproximadamente para favorecer la decantación de los cocolitos sobre el cubre. Es importante que la temperatura ambiente no sea muy elevada o parte de la disolución podría evaporarse rápidamente provocando una distribución heterogénea de los cocolitos sobre el cubre.

9. Extraer el líquido sobrante mediante capilaridad con ayuda de pequeñas tiras de papel secante.

10. Dejar secar la placa de Petri unos minutos.

11. Retirar el cubre del fondo de la placa y posarlo sobre una placa térmica a $80-90{ }^{\circ} \mathrm{C}$ aproximadamente.

12. Poner sobre el cubre una gota de bálsamo de Canadá disuelto al $30 \%$ con xileno y cubrirlo con el porta con cuidado para que no queden burbujas.

13. Etiquetar la muestra y dejarla en la estufa a $50{ }^{\circ} \mathrm{C}$ aproximadamente durante al menos 24 horas para que el bálsamo se seque, elimine las posibles burbujas y selle la muestra. 


\section{ANEXO III.-PROCEDIMIENTOS ADOPTADOS PARA LOS ANÁLISIS DE ISÓTOPOS DE OXÍGENO}

\section{A) Protocolo de limpieza de viales}

1. Preparar dos disoluciones con ácido nítrico al $10 \%$ (9 partes de agua destilada y una parte de $\mathrm{HNO}_{3}$ ).

2. Limpiar una micropipeta con la disolución preparada bajo la campana de extracción y siempre con los guantes puestos.

3. Llenar cada vial y su tapón con esta disolución con ayuda de la micropipeta y con cuidado de no dejar burbujas en el fondo.

4. Meter los viales en posición vertical en un bote con la disolución de ácido nítrico. Tapar el bote y dejarlo bajo la campana toda la noche.

5. Transcurrido ese tiempo, extraer la disolución del bote, dejando los viales dentro.

6. Llenar el bote con agua destilada, enjuagar y extraer el agua.

7. Limpiar la micropipeta con agua destilada y uno por uno enjuagar dos veces los viales y su tapón. Depositarlos en un cuenco cubierto con dos capas de papel poco fibroso.

8. Limpiar la micropipeta con agua miliQ y uno por uno, enjuagar dos veces los viales que ya habían sido enjuagados con agua destilada.

9. Sacudir bien los viales para que se sequen antes y dejarlos en otro cuenco cubierto con papel.

10. Meter el cuenco con los viales ya limpios bajo la campana de extracción, cubierto con un papel.

\section{B) Preparación de las muestras}

1. Pesar el sedimento seco disponible en cada muestra y anotar el resultado.

2. Echar el sedimento en un vaso de precipitados rotulado con el nombre de la muestra y verter agua del grifo (aproximadamente $300 \mathrm{~mL}$ ).

3. Someter a ultrasonidos el tamiz de $63 \mu \mathrm{m}$ (este paso se ha de realizar entre muestra y muestra para limpiar el tamiz).

4. Verter el contenido del vaso de precipitados sobre el tamiz mientras se deja correr sobre éste el agua del grifo también, y lavarlo poco a poco hasta vaciar el vaso.

5. El sedimento recogido en el tamiz se deposita con ayuda de agua destilada en un cuenco de cerámica previamente rotulado con el nombre de la muestra.

6. Dejar el cuenco en la estufa toda la noche a $50-60{ }^{\circ} \mathrm{C}$ aproximadamente.

7. Transcurrido ese tiempo, sacar el cuenco de la estufa y dejarlo a temperatura ambiente.

8. Para cada muestra, se han de etiquetar dos cajitas, una para la facción $>63 \mu \mathrm{m}$ y otra para la fracción $>150 \mu \mathrm{m}$. 
9. Colocar el tamiz de $150 \mu \mathrm{m}$ sobre un cuenco vacío rotulado con el nombre de la muestra. Dar ligeros golpecitos al tamiz contra las paredes del cuenco, habiendolo tapado previamente con un vidrio de reloj para evitar la pérdida de material. En el cuenco caerá la fracción de $>63 \mu \mathrm{m}$ y en el tamiz quedará la fracción de $>150 \mu \mathrm{m}$.

10. Pesar ambas fracciones en la báscula de precisión y depositar en sus respectivas cajas.

\section{C) Picking y crushing}

El picking es el proceso por el cual, con ayuda de un pincel y unas gotas de agua destilada, se seleccionan bajo una lupa los especímenes de la especie (o especies) de interés que se utilizarán en el análisis de isótopos estables. Para los análisis de isótopos estables realizados en esta tesis doctoral se "picaron" como mínimo 25 especímenes bien preservados de G. bulloides de la fracción $>150 \mu \mathrm{m}$.

El crushing de los foraminíferos se realiza para que el proceso de limpieza sea más exhaustivo. Esta técnica consiste en abrir las cámaras de los foraminíferos. Para ello se colocan los especímenes de una misma muestra sobre un porta y con otro porta se aprietan hasta que se rompen sus cámaras, con cuidado de no pulverizarlas.

\section{D) Protocolo de limpieza previa al análisis de isótopos estables}

1. Bajo la campana de extracción de gases, añadir unas gotas de metanol a cada vial, llenando del todo la parte cónica (unas 3 gotas).

Someter a ultrasonidos durante un minuto colocando encima una gradilla para evitar que los tapones salten.

2. Limpiar la punta de la pipeta dos veces con $\mathrm{HNO}_{3}$ al $10 \%$ y tres veces con agua miliQ. Retirar al máximo el excedente de metanol con la pipeta Pasteur, siempre bajo la vitrina de extracción.

3. Dejar los viales abiertos bajo la vitrina de gases entre dos y tres horas para permitir que se sequen las muestras.

4. Es conveniente revisar el contenido de los viales bajo la lupa ya que en ocasiones pequeñas fibras pueden haberse introducido en los mismos. 


\section{ANEXO IV.-PROCEDIMIENTOS ADOPTADOS PARA EL ANÁLISIS DE COMPUESTOS ORGÁNICOS}

\section{A) Pesada de la muestra:}

1. Sacar las muestras del congelador y dejarlas en la liofilizadora durante 12-18 horas.

2. Una vez liofilizadas, congelarlas hasta que se vayan a utilizar para su análisis.

3. Sacar las muestras y dejarlas una hora a temperatura ambiente.

4. Poner la muestra en dos capas de papel de aluminio y aplastarla con un mortero para que quede el sedimento fino, posteriormente homogeneizar con la espátula.

5. Pesar 2,5 g aproximadamente y echarlos en un tubo de centrífuga debidamente etiquetado. Limpiar la espátula con diclorometano entre muestra y muestra.

6. Tapar con papel de aluminio y cerrar con su tapón.

7. Adicionalmente, también se rotulará un tubo para el blanco que ha de seguir los mismos pasos que las muestras de sedimento.

\section{B) Preparación del patrón:}

1. Sacar el patrón del congelador y dejar a temperatura ambiente durante 10-20 minutos y rotular tantos viales como patrones se vayan a preparar.

2. Vortear el patrón y limpiar la aguja de la jeringa de la pipeta electrónica con tolueno, con dos tubos de ensayo. Limpiarla 10 veces con el contenido de un tubo y 10 veces con el contenido del otro tubo.

3. Añadir en cada vial $10 \mu \mathrm{L}$ de patrón (en este caso fue el 29B) con ayuda de la pipeta.

4. Dejar evaporar a sequedad en el SpeedVack durante 30 minutos.

5. Añadir $50 \mu \mathrm{L}$ de BSTF habiendo limpiado la jeringa previamente.

6. Tapar cada vial con el séptum y el tapón.

7. Agitar cada vial en el vórtex y el patrón ya está listo para ser inyectado en pasos posteriores.

\section{C) Extracción con cloruro de metileno $\left(\mathrm{CH}_{2} \mathrm{Cl}_{2}\right)$ :}

1. Si el patrón preparado en el paso B) se encuentra en el congelador, sacarlo y esperar a que esté a temperatura ambiente (aproximandamete 30 minutos).

2. Vortearlo durante unos segundos.

3. Limpiar la jeringa con tolueno, repartido en dos tubos de ensayo. Con el contenido del primer tubo se ha de limpiar 10 veces y después otras 10 veces con el contenido del segundo tubo.

4. Destapar los tubos con el sedimento y añadirles $10 \mu \mathrm{L}$ de patrón con la jeringa, incluido el blanco.

5. Añadir $6 \mathrm{~mL}$ de $\mathrm{CH}_{2} \mathrm{Cl}_{2}$ y volver a tapar con el papel de aluminio y el tapón. 
6. Agitar el tubo en el vórtex durante 15 segundos.

7. Dejar el tubo junto a otros ya vorteados en el baño de ultrasonidos durante 15 minutos, teniendo en cuenta que a los 7 minutos hay que sacarlos y voltearlos para que se mezcle el sedimento.

8. Mientras tanto, limpiar el evaporador pasando un tubo de $\mathrm{CH}_{2} \mathrm{Cl}_{2}$ por cada aguja.

9. Al terminar los ultrasonidos, volver a vortear y centrifugar los tubos (esta vez sin el tapón pero tapados con el papel de aluminio) durante 5 minutos.

10. Mientras tanto, preparar las pipetas y los tubos de ensayo en los que se pondrá el extracto. Cubrir la gradilla con papel de aluminio y evitar que las pipetas se toquen entre sí en todo momento para evitar la contaminación entre muestras. Preparar también una gradilla con tubos numerados y el nombre de la tanda en la gradilla.

11. Extraer con una pipeta Pasteur la fase líquida de cada tubo y depositarla en otro tubo.

12. Abrir la llave de $\mathrm{N}_{2}$ y las clavijas. Poner a evaporar los tubos con las muestras con corriente de $\mathrm{N}_{2}$ hasta que quede un dedo de muestra. Cerrar la llave de $\mathrm{N}_{2}$.

13. Volver a repetir todo el procedimiento desde el paso número 5 hasta la evaporación con $\mathrm{N}_{2}$ un total de 3 veces, de forma que se realicen 3 extracciones en total.

14. Tras la última extracción, dejar evaporar a sequedad, es decir, por completo, en atmósfera de $\mathrm{N}_{2}$.

15. Añadir con la micropipeta $100 \mu \mathrm{L}$ de tolueno y agitar en el vórtex.

16. Añadir $2 \mathrm{~mL}$ de potasa metabólica al $10 \%$.

17. Agitar en el vórtex y dejar los tubos en ultrasonidos durante 15 minutos.

18. Dejar reaccionar toda la noche.

\section{D) Extracción con hexano $\left(\mathrm{C}_{6} \mathrm{H}_{14}\right)$}

1. A la mañana siguiente, añadir a cada tubo 4 o 5 gotas de agua miliQ para parar la reacción.

2. Añadir $2 \mathrm{~mL}$ de $\mathrm{C}_{6} \mathrm{H}_{14} \mathrm{y}$ poner en el vórtex y en el baño de ultrasonidos durante 5 minutos aproximadamente.

3. Preparar las pipetas y los tubos de ensayo con la gradilla como en el paso C) 10.

4. Tras el baño de ultrasonidos, volver a vortear durante 15 segundos.

5. Extraer el extracto y ponerlo en un tubo de ensayo (identificado con "H").

6. Añadir $2 \mathrm{~mL}$ de $\mathrm{C}_{6} \mathrm{H}_{14}$ y vortear unos segundos.

7. Realizar la segunda extracción.

8. Añadir otros $2 \mathrm{~mL}$ de $\mathrm{C}_{6} \mathrm{H}_{14}$, vortear unos segundos y realizar la tercera extracción.

9. Acumular el extracto en el mismo tubo, en total 3 extractos.

10. Tras la tercera extracción, evaporar en atmósfera de $\mathrm{N}_{2}$ hasta que quede un dedo. 
11. Añadir $2 \mathrm{~mL}$ de agua miliQ para arrastrar los restos de potasa, agitar en el vórtex y extraer la fase orgánica a los tubos de ensayo identificados con "H". Se ha de pipetear por encima de la línea de burbujas.

12. Añadir $2 \mathrm{~mL}$ de $\mathrm{C}_{6} \mathrm{H}_{14}$, vortear y extraer.

13. Realizar el paso anterior una vez más, un total de tres veces (una extracción con agua miliQ y dos extracciones con $\left.\mathrm{C}_{6} \mathrm{H}_{14}\right)$.

14. Dejar evaporar a sequedad.

\section{E) Derivatización}

1. Identificar los viales con el nombre del testigo, la profundidad y el código del tubo de ensayo.

2. Añadir a los tubos de ensayo $100 \mu \mathrm{L}$ de solución $\mathrm{CH}_{2} \mathrm{Cl}_{2} / \mathrm{C}_{6} \mathrm{H}_{14}(8: 2)$.

3. Vortear dentro de la campana para recuperar lo que pueda haber en las paredes y trasvasar a un vial debidamente etiquetado con el nombre de la muestra con ayuda de una pipeta.

4. Una vez vaciado el tubo volver a verter la solución con la pipeta pero esta vez enjuagando también las paredes del tubo para recuperar el máximo de material posible. Durante la tercera o cuarta vez coger todo lo posible y transvasar al vial, que ha de haberse llenado no más de tres cuartas partes.

5. Repetir dos veces más desde el paso número 2 hasta el 5 hasta haber añadido $300 \mu \mathrm{L}$ en total (realizar el proceso 3 veces en total).

6. Poner a secar en el SpeedVac hasta su sequedad total (aproximadamente 30 minutos).

7. Poner los tapones con sus septums a cada uno de los viales.

8. Cambiar la jeringuilla de la pipeta electrónica y poner la aguja de BSTFA. Limpiarla como siempre con tolueno como se ha hecho en pasos anteriores.

9. Añadir con la pipeta electrónica $50 \mu \mathrm{L}$ de BSTFA en cada vial (incluido el blanco) y en el patrón (donde ya había $10 \mu \mathrm{L}$ de patrón que ya se habrán evaporado y a los que se le añaden otros $50 \mu \mathrm{L}$ para obtener los $60 \mu \mathrm{L}$ finales).

10. Vortear los tubos unos segundos. 


\section{ANEXO V.-LISTA DE ACRÓNIMOS}

Recopilación de acrónimos y abreviaturas utilizados en esta tesis doctoral. Para el significado de los acrónimos anglosajones se presenta, entre paréntesis, su equivalente en castellano cuando éste también ha sido utilizado en el texto.

AC: $\quad$ Alboran cooling events (eventos de enfriamiento de Alborán)

AC: $\quad$ Algerian Current (corriente argelina)

AJ: $\quad$ Atlantic Jet (chorro atlántico)

AMS: $\quad$ accelerator mass spectrometry

APC: $\quad$ periods of continental aridity

AW: $\quad$ Atlantic Water (agua atlántica)

B-A: $\quad$ Bølling-Allerød

BP: $\quad$ Before Present

CCA: Canonical Correspondance Analyses (análisis canónico de correspondencias)

CSIC: $\quad$ Consejo Superior de Investigaciones Científicas

CTD: $\quad$ Conductivity-Temperature Depth

DCA: Detrended Correspondance Analyses (análisis de correspondencias sin tendencia)

DMS: dimetilsulfuro

DW: $\quad$ Deep Water (agua profunda)

EAG: $\quad$ Eastern Anticyclonic Gyre (giro anticiclónico oriental)

ENSO: $\quad$ El Niño-Southern Oscillation (Oscilación del Sur de El Niño)

FA: $\quad$ freshwater advection events

H2 y H1: estadiales asociados con los Eventos Heinrich 2 y 1

IACP: Intra-Alleröd Cold Period

IPCC: Intergovernmental Panel on Climate Change (Panel Intergubernamental sobre el Cambio Climático)

LGM: $\quad$ Last Glacial Maximum (Último Máximo Glacial)

LIW: $\quad$ Levantine Intermediate Water (Agua Intermedia Levantina)

LPZ: $\quad$ lower photic zone (zona fótica inferior)

M8-M0: $\quad$ Minorca Events

MAT: $\quad$ Modern Analog Technique (técnica de análogos modernos)

MAW: $\quad$ Modified Atlantic Water (Agua Atlántica Modificada)

MOW: $\quad$ Mediterranean Outflowing Water (Flujo de Salida Mediterráneo)

MW: $\quad$ Mediterranean Water (Agua Mediterránea)

N: $\quad$ absolute abundance of coccoliths (abundancia absoluta de cocolitos) 
NADW: North Atlantic Deep Water (Agua Profunda Noratlántica)

NAO: $\quad$ North Atlantic Oscillation (Oscilación del Atlántico Norte)

NAR: Nannofossil Accumulation Rate (tasa o flujo de acumulación de nanofósiles)

NC: $\quad$ Northern Current (corriente del Norte)

ODV: $\quad$ Ocean Data View

ORL: $\quad$ Organic-Rich Layer

PC1: $\quad$ First Principal Component (primera componente principal)

PCA: $\quad$ Principal Component Analyses (análisis de componentes principales)

r: $\quad$ coeficiente de correlación muestral

$\mathbf{R}^{2}$ : $\quad$ coefficient of determination (coeficiente de determinación)

RMSEP: root-mean square error of prediction (error cuadrático medio de predicción)

SD: $\quad$ Standard Deviation (desviación estándar)

SMS: $\quad$ salinidad marina superficial

SSS: $\quad$ Sea surface salinity (salinidad marina superficial)

SST: $\quad$ Sea surface temperature (temperatura superficial del mar)

ST: $\quad$ sedimentation rate (tasa de sedimentación)

T1a: $\quad$ Termination 1a (Terminación 1a)

T1b: $\quad$ Termination $1 b$ (Terminación 1b)

TMF: $\quad$ Temperate Mediterranean Forest

TS: $\quad$ tasa de sedimentación

TSM: $\quad$ temperatura superficial marina

UPZ: $\quad$ upper photic zone (zona fótica superior)

VPDB: $\quad$ Vienna Pee Dee Belemnite

WAG: Western Anticyclonic Gyre (giro anticiclónico occidental)

WA-PLS: weighted-averaging partial-least square regression (regresión parcial por mínimos cuadrados ponderados)

WMDW: Western Mediterranean Deep Water (Agua Mediterránea Profunda occidental)

WOA: World Ocean Atlas

WWG: Warm-water Group (grupo de aguas cálidas)

YD: $\quad$ Younger Dryas

YDa: $\quad$ Phase a of the Younger Dryas (primera fase del Younger Dryas)

YDb: $\quad$ Phase b of the Younger Dryas (segunda fase del Younger Dryas)

ZFI: $\quad$ zona fótica inferior

ZFM: $\quad$ zona fótica media

ZFS: $\quad$ zona fótica superior 


\section{ANEXO VI.-ÍNDICE GENERAL}

Alcohol index/ índice alcohol (n-hexacosan-1-ol index): 63, 84, 91, 93, 95, 98, 121, 124, 126, 127,131

Alkenones/ alquenonas: 20, 34, 61, 62, 84, 90, 98, 121,

Atlantic Jet (AJ): 2, 4, 7, 9, 78, 79, 96, 99, 116, 117, 127, 130, 131, 132, 134

Atlantic Water/ Agua Atlántica (AW):2, 7, 76, 77, 78, 79, 92, 94, 96-101, 117, 149, 167-172, $183-185$

Bølling-Allerød (B-A): 85, 94, 96, 169

Freshwater advection events (FA): 170-171

H2 y H1/ stadials associated with Heinrich Events 2 and 1: 85, 94, 91-95, 167

Last Glacial Maximum/ Último Máximo Glacial (LGM): 93-95, 167

Lower Photic Zone/ zona fótica inferior (LPZ): 27, 125, 127-128

Modern Analog Techniquel técnica de análogos modernos (MAT): 66, 84, 146-147, 156-157, 159-167

North Atlantic Oscillation/ Oscilación del Atlántico Norte (NAO): 10-11, 114-116, 118-119, $132-133,185$

Organic-Rich Layer (ORL): 94-97, 169, 184-185

Oxygen isotopes/ isótopos de oxígeno $\left(\delta^{18} \mathrm{O}\right): 61,82,88,91,121-122,192$

Reworked specimens/ especímenes retrabajados: 20, 82, 92-93, 131, 152

Sea surface salinity/ salinidad marina superficial (SSS): 146-149, 157-159, 161-172

Sea surface temperature/ temperatura superficial del mar (SST): 83-83, 88-91, 94-100

Termination 1a/ Terminación 1a (T1a): 85, 94-96, 183

Termination 1b/ Terminación 1b (T1b). 84-85, 98, 183

Total concentration of alkenones/ concentración total de alquenonas $\left(C_{37}\right.$ alkenones): 62, 95, 96, $98,121,124,126,127,169$

Transfer function/ función de transferencia: 65-67, 146-149, 156, 159, 164-165, 172, 185-186

Upper Photic Zone/ zona fótica superior (UPZ): 27, 128

Western Anticyclonic Gyre/ giro anticiclónico occidental (WAG): 3, 6-8, 78-79, 117-118, 127 , 130,134 
Weighted-averaging partial-least square regression/ regresión parcial por mínimos cuadrados ponderados) (WA-PLS): 66-67, 146-147, 156-171

Western Mediterranean Deep Water/ Agua Mediterránea Profunda occidental (WMDW): 5-6, $48,79,114-115,117,128-134,184$

Younger Dryas (YD): 94, 97, 170, 184-185 


\section{Agradecimientos}

Ha llegado el momento de dar las gracias a todas las personas que han contribuido de una forma u otra, en mayor o menor medida, a la realización de esta tesis doctoral y a su resultado final.

Gracias a los Dres. Jose Abel Flores y Francisco Javier Sierro por supervisar esta tesis. Abel, gracias por darme la oportunidad de embarcarme en el apasionante mundo de la ciencia, por dejarme tomar la iniciativa, por confiar en mí siempre, por todos los lugares maravillosos que he conocido gracias a ti y por ese optimismo científico con el que una y otra vez demuestras tener razón. Gracias Paco por tener siempre un rato para darme tu visión científica, pero sobre todo, por tu fantástico sentido del humor y la cantidad de anécdotas con las que los becarios siempre soñamos escribir un libro. Lines, gracias por valorarme y confiarme a tus biólogos, por estar siempre dispuesta a echar una mano y por poner al GGO algo de ese carácter del norte que yo tantísimo echo de menos cuando estoy fuera de mi tierra.

Gracias a mis compañeros y colegas. A los que estaban cuando llegué, a los que vinieron de paso y a las nuevas incorporaciones. A Iván, qué decirte que quepa aquí... Gracias por todas las carcajadas, por ayudarme tantísimo y por ser tan buena gente debajo de esas gafotas de pasta; ojalá la ciencia contara con más científicos tan valiosos, críticos y entregados como tú. Gracias a Miguel Ángel ... este año he echado en falta tu risilla maliciosa y tus cosas de Jimmy Neutrón. A Eloy, por prestarte siempre a echar una mano en todo lo que hiciera falta; todo es mucho más fácil si al llegar a un sitio nuevo hay compañeros como tú. Gracias Aleix por todo lo que me has hecho reír en la oficina. A Marta por las veces que saca de paseo su humor fricazo iy por las inmersiones lingüísticas en cacereño! Gracias Anita, ese soplo de aire fresco en forma de risa constante y clase de buena gente con la que da gusto trabajar. A Margarita, por cuidar de todos en tantos sentidos, aunque de todos, mi favorito es el que incluye tortas y arepitas. A Bas, por transmitir esa buena vibra. A Marlies, por hacer de la oficina un sito aún más agradable. A Gloria, por tu simpatía, y tu trabajo tan meritorio que es toda una inspiración. A Roci, porque es un gustazo entrar en el laboratorio y saber que tú vas a estar ahí derrochando majeza. Y gracias a Jose Ignacio, porque es un placer saber que aún quedan naturalistas de los de antes en este mundo.

Gracias a la Dra. Beatriz Mouriño-Carballido, por todo, por abrirme una puerta a este mundo fascinante, dejarme probar y animarme a dar mis primeros pasitos. Una vez me 
dijiste que "es un lujo que te paguen por hacer lo que te apasiona", no lo he olvidado y con el tiempo lo he comprobado por mí misma. GRACIAS.

Gracias a la Dra. Isabel Cacho de la Universidad de Barcelona y al Dr. Joan Grimalt del Instituto de Diagnóstico Ambiental y Estudios del Agua (CSIC, Barcelona) por hacerme un hueco en sus respectivos laboratorios. Gracias a la Dra. Belén Martrat por supervisar mi estancia en el CSIC de Barcelona, siempre tan amable y dispuesta a echarme una mano en todo, durante y después, igracias! Y gracias a ese grupo tan majo (Bibi, Marta, Óscar, Aleix, David, Anuar, Yolanda, etc.) que tantos buenos ratos me hicieron pasar entre extracción y extracción. Gracias a la Dra. Belén Alonso por hacerme un hueco en el repositorio de muestras del fondo marino del Instituto de Ciencias Marinas (CSIC, Barcelona). Me acordé mucho de Forest Gum y su cajita de bombones muestreando esos testigos oceánicos, pero mereció mucho la pena. Gracias a la Dra. Gemma Ercilla, por echarme más de una mano desinteresada a vuelta de email con la morfología de mi zona de estudio. Gracias al Dr. Guillermo Francés de la Universidad de Vigo, por tu disposición para ayudarme en lo que hiciera falta. I would also like to thank Dra. Antje Voelker, for giving me the opportunity to get on board the García del Cid and enjoying oceanography on the field. Herzlich bedanken möchte ich mich bei Dr. Martin Grosjean von der Universität Bern. Er ermöglichte mir, meine Dissertation bei ihm und seiner fantastischen Forschungsgruppe im Rabmen eines Forschungsaufenthaltes weitervuentwickeln. Thank you so much Tobi, Iván, Krystina, Christopher, Valentin and Ben, with who I had the real pleasure of enjoying my research stay in Switzerland.Thank you guys for the warm welcome, the bicycles, the BBQs...it was great to work with you! Y cómo no, a Iván y Olaia, por hacer de su casa y su gente las nuestras y jacompañarnos a batir los tiempos de todas las rutas del libro verde de Oli!

Gracias a Julio, mi profe de dibujo en cuboespaciocreativo, por ayudarme con la ilustración de la tesis, y enseñarme siempre con tanta paciencia y una sonrisa. ¡Pinturas de palo forever!

Gracias a mis charritos y apegados: María, Hugueles, Elena, Mati y David. Por las ganas de cambiar el mundo, las risas, los lunes de aguas, los audios, las pedradas desde medio campo que se convierten en triplazos y ipor la próxima! Gracias a Esther, el tesoro que me llevé de Vigo y que no pienso devolver. ¡Construye ese establo de una vez!, que me muero por verte la cara cuando aparezca con Blanquita en brazos.

Y cómo no, ahora le toca el turno a la banda sonora de fondo de esta tesis. A todos los que, sin tener ni idea de lo que es un cocolito ni una oceanógrafa, son las mejores personas de las que me he rodeado. 
Gracias a mi familia, por apoyarme siempre, porque a pesar de padecer todos mis viajes, de verme regresar a sabiendas de que no voy a quedarme, seguís viniendo a recibirme a la estación, sin importar la hora del día o de la noche. A mi tía Toñi y mi prima Eva, a las que tanto quiero y se lo digo poco. Gracias Mamá por demostrarme siempre estar orgullosa de mí; espero que nunca dejes de estarlo, porque hay mucho de ti en todo lo que hago bien. Gracias Tati, porque me basta con silbar si hay monstruos debajo de mi cama, y porque te sobra corazón para que los demás no nos pongamos tristes. Te quiero, y te admiro, y vice versa. Gracias a Víctor, el hermano que nunca tuve y digno competidor por las sobras de los domingos. A Jorge, ese torbellino que lo llena todo con su risa; ojalá por mucho que pase el tiempo sigas gritando mi nombre cada vez que llame a la puerta. ¡Y al pequeño Leo! ¡Qué ganas de verte crecer!

Gracias a mi otra familia de fantásticas criaturas: Yeray, Rebe, Víctor, Leti, Javi, Paju, Pas, Keko, Hispano, Helen, Dani y Pe. Cómo pasa el tiempo y qué feliz me hace ver que seguimos ahí, como el primer día en algún banco del parque Santiago echándonos unas risas. Estáis para lo malo y lo más importante, sois responsables de gran parte de lo bueno. Sois muy importantes para mí y en cada uno de vosotros encuentro una inspiración: la alegría, la sencillez, la superación, la honestidad, el wanderlust, la lucha, el "no risk, no fun", la nobleza y una lista infinita de cosas que no caben aquí. En fin, me gusta pensar que soy también un poco de vosotros e inevitablemente eso me llena de orgullo. Gracias por hacerme sentir tan afortunada.

Gracias a la maravillosa familia de Hugo: a los abuelos, a Loli, a Diego, a July y a Iñaki e Iván que también son familia. Gracias por tratarme siempre con tanto cariño y hacer que sea un lujazo ir a León. Gracias inmensamente a Merce, por todo, por mimarme tantísimo (iiiy!!! por hacer que nunca me falte un túper de esas lentejas que tanto me alegran el día); e infinitamente a Pablo, porque me hacías una falta inmensa. Y en definitiva, gracias a ambos por apoyarnos siempre en todas nuestras decisiones, en las sensatas, y en las que no lo son tanto.

Cómo no dedicar aquí unas líneas a Hugo, mi compañero y siempre la mejor de las personas que han pasado por mi estima. Gracias por hacer del pasado el lugar al que no me canso de echar la vista, del presente bendita felicidad y del futuro una hermosa taquicardia. Darte las gracias por todo no es más que parte de la deuda que tengo contigo, si es que existen deudas en el querer más infinito. 





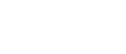
(
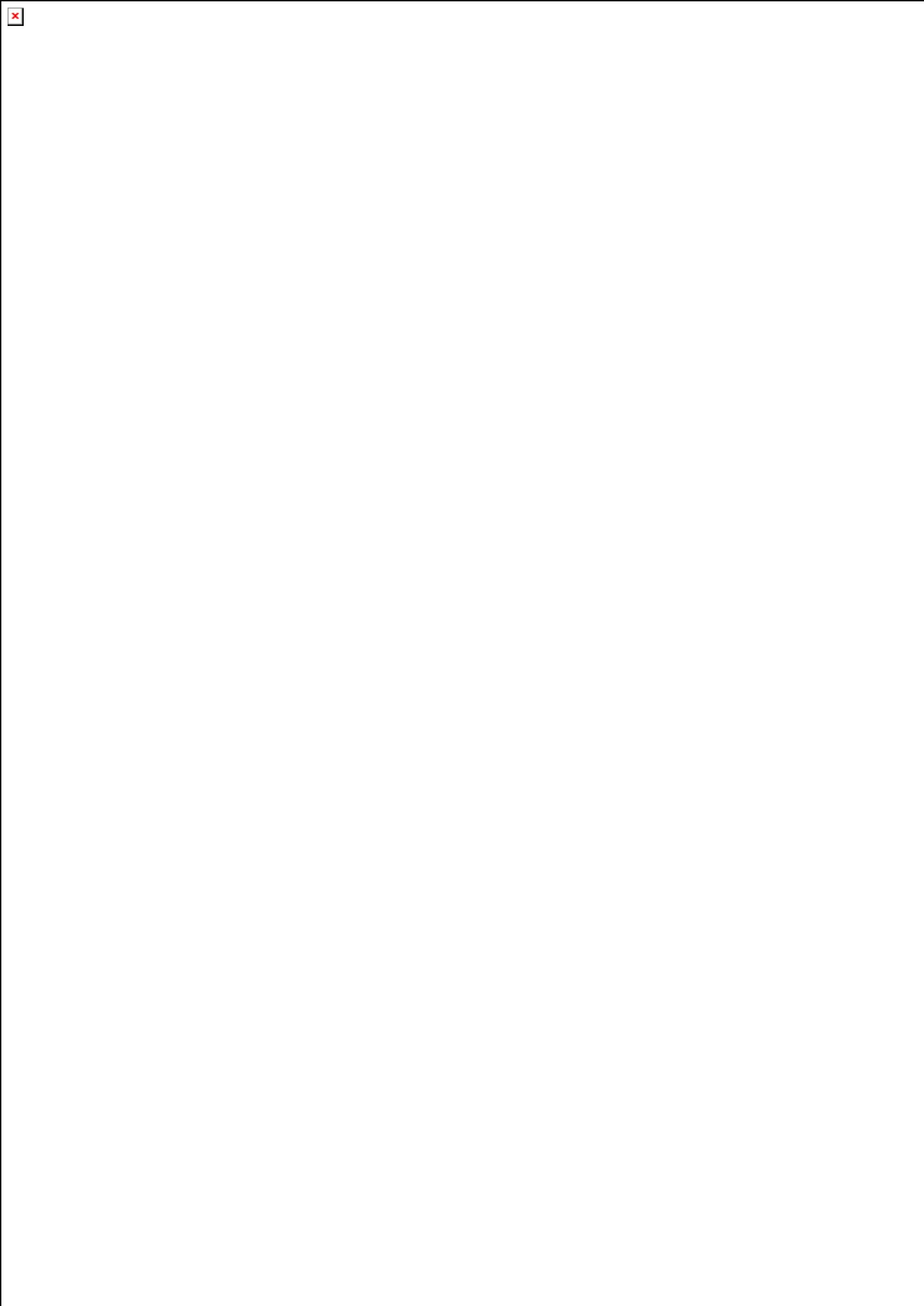